\title{
Synthesis of Proline Analogues via Rh-Catalyzed Asymmetric Conjugate Addition
}

\author{
Emma K. Edelstein, ${ }^{\star *}$ Danica A. Rankic, ${ }^{\ddagger}$ Caroline C. Dudley, ${ }^{\star}$ Spencer E. McMinn ${ }^{\dagger}$ and \\ Donovon A. Adpressa
}

\footnotetext{
Eepartment of Process Research and Development, Merck \& Co., Inc., Boston, Massachusetts 02115, United States 'Department of Discovery Chemistry, Merck \& Co., Inc., Boston, Massachusetts 02115, United States

§. Present Address: Pfizer Worldwide Research and Development, Groton, Connecticut 06340, United States

*Corresponding author: emma.edelstein@merck.com
}

\section{Supporting Information}

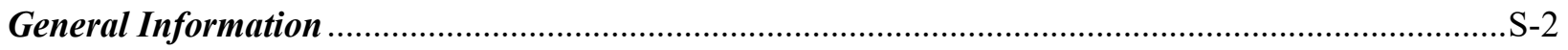

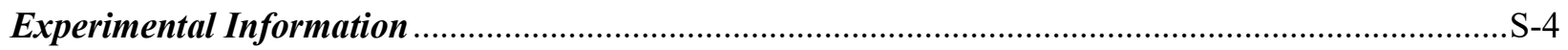

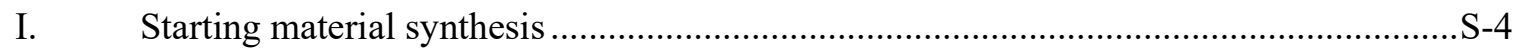

II. Preliminary results and chiral ligand screen .............................................................

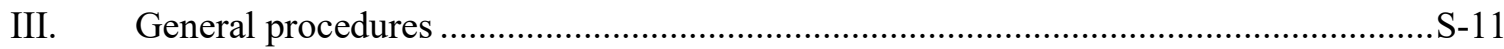

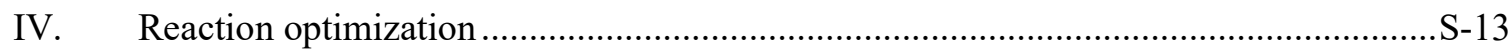

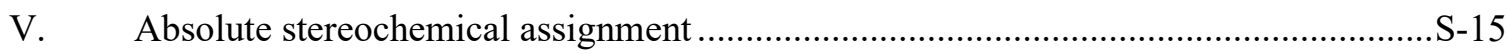

VI. Characterization of reaction products and analysis of stereochemistry …....................S-26

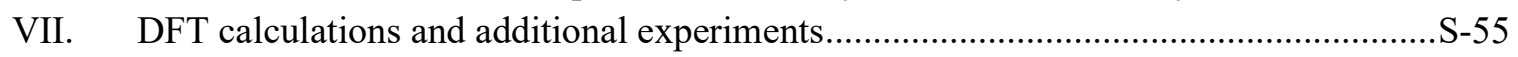

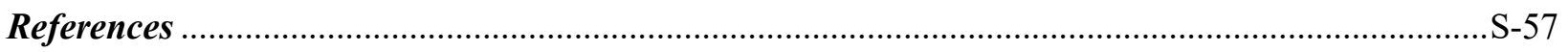

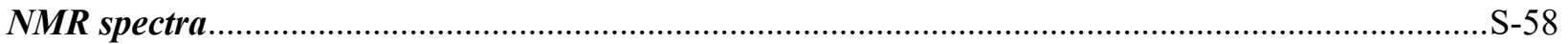




\section{General Information}

Reactions were performed in scintillation vials with Teflon septa unless otherwise specified. Reactions were monitored by liquid chromatography/mass spectrometry (LCMS), ultra performance liquid chromatography (UPLC), or by thin-layer chromatography (TLC) on silica gel60 F254 plates (EMD) and visualized with UV light $(254 \mathrm{~nm})$. Flash chromatography was performed using prepacked RediSep gold Rf silica gel columns on a Teledyne Isco CombiFlash Rf automated chromatography system. Organic solutions were concentrated under reduced pressure on a Buchi rotary evaporator. Tetrahydrofuran (THF), 2-Methyl tetrahydrofuran (2-MeTHF), toluene (PhMe or Tol), 1,4-dioxane, and acetonitrile (MeCN), were purchased from Sigma-Aldrich in Sure-Seal ${ }^{\mathrm{TM}}$ bottles and used as received. $\mathrm{CDCl}_{3}, \mathrm{CD}_{3} \mathrm{OD}$, DMSO- $d_{6}$, acetone- $d_{6}$ and Acetonitrile- $d_{3}$ were purchased from Cambridge Isotope Laboratories, Inc. and used as received. $(R, R)-\mathrm{Ph}-\mathrm{BPE}-\mathrm{Rh}$ (cas 528565-84-6), $(S, S)$-Ph-BPE-Rh (cas 849950-53-4), $\mathrm{Rh}(\mathrm{nbd})_{2} \mathrm{BF}_{4}$ (cas 36620-11-8), were purchased from Strem Chemicals and used as received. The boronic acid substrates, ligands, and all other reagents were purchased from commercial sources and used as received.

Compounds were analyzed by ${ }^{1} \mathrm{H},{ }^{13} \mathrm{C}$, and ${ }^{19} \mathrm{~F}$ NMR, where appropriate. All NMR spectra were recorded on a Bruker AVANCE III NMR spectrometer $\left(600 \mathrm{MHz}\right.$ for ${ }^{1} \mathrm{H}, 151 \mathrm{MHz}$ for ${ }^{13} \mathrm{C}$, and $564 \mathrm{MHz}$ for ${ }^{19} \mathrm{~F}$ ) equipped with a $1.7 \mathrm{~mm}$ MicroCryoProbe ${ }^{\mathrm{TM}} \mathrm{TXI}$ probe at the temperature indicated on the spectra. All NMR chemical shifts are reported in parts per million (ppm) relative to DMSO-d6, $2.50 \mathrm{ppm}$ for ${ }^{1} \mathrm{H}$ NMR and 39.52 for ${ }^{13} \mathrm{C} \mathrm{NMR}$ or $\mathrm{CDCl}_{3}, 7.26$ ppm for ${ }^{1} \mathrm{H} \mathrm{NMR}$ and 77.16 for ${ }^{13} \mathrm{C}$ NMR. Signals are quoted as singlet (s), doublet (d), triplet (t), quartet (q), quintet (quint), and multiplet (m). Coupling constants are reported in Hz. All data were analyzed using MestReNova v12.1 (Mestrelab Research S.L.). Most compounds exist as approximately 1:1 rotamer forms in DMSO-d6 under the conditions analyzed and are reported as such unless specifically indicated. In the instances where dr approaches less than 3:1 only the trans-product is tabulated.

High-resolution mass spectrometry (HRMS) data were recorded on a Waters Acquity UPLC equipped with diode array detector and a Xevo G2-XS Qtof MS instrument in ESI+ (electrospray) ionization mode. UPLC-HRMS was performed utilizing a Water C18 BEH 2.1x100mm column with 8 minute gradient method starting at $10 \%$ water with $0.1 \%$ TFA held isocratically for 30 seconds followed by a 6 minute gradient to $99 \%$ acetonitrile and held isocratically for 1 minute before re-equilibrating at $10 \%$ aqueous for 30 seconds.

SFC-MS analytical experiments were performed on a Waters Acquity UPC ${ }^{2}$ system equipped with a binary solvent manager pump, FL sample manager, two $30 \mathrm{~S}$ column ovens, backpressure regulator, photodiode array detector (PDA), Qda mass detector, and evaporative light scattering (ELS) detector. Data acquisition, instrument control, and chromatogram processing was performed with Empower 3. Racemic standards were used to develop isocratic methods to determine the diastereomeric ratio (dr) and 
enantiomeric excess (ee). For method development, the $\mathrm{UPC}^{2}$ was equipped with the following columns. AD-3, AS-3, OJ-3, OD-3, IC, ID, IF, and IG from Chiral Technologies (West Chester, PA); Lux-2, Lux-3, and Lux-4 from Phenomenex (Torrance, CA); CCA, CCC, CCO from ES Industries (West Berlin, NJ); (R,R)-Whelk-O1 from Regis Technologies (Grove, IL). All columns were $3 \mu \mathrm{m}$ particle size and $4.6 \mathrm{mmX100mm} \mathrm{except} \mathrm{for} \mathrm{the}(R, R)$-Whelk-O1 which was $3.5 \mu \mathrm{m}$ particle size. The mobile phase, comprised of a mixture of $\mathrm{CO}_{2}$ and Methanol with $0.1 \% \mathrm{NH}_{4} \mathrm{OH}$ as the modifier, was delivered with a flow rate of $3.5 \mathrm{ml} / \mathrm{min}$. The gradient profile was as follows: Initial conditions of $5 \%$ modifier ramped to $60 \%$ modifier over 3 minutes, hold at $60 \%$ modifier for 0.75 minutes, return to initial conditions at 4 minutes. Each column was allowed a 0.5 minute equilibration with initial conditions prior to analysis. Additional make-up solvent, a $0.1 \%$ formic acid solution in $95 / 5 \mathrm{v} / \mathrm{v}$ methanol/water, was delivered at a flow rate of $0.5 \mathrm{ml} / \mathrm{min}$ by a Waters isocratic solvent manager (ISM) pump. Column temperature was set to $40^{\circ} \mathrm{C}$ and BPR was set to 2000psi.

Preparative SFC was performed using a Sepiatec Prep SFC 100 system, which included a highpressure $\mathrm{CO}_{2}$ pump, a high-pressure cosolvent pump, a photodiode array detector (PDA) detector, an automatic backpressure regulator, and a valve to collect up to eight fractions (Sepiatec $\mathrm{GmbH}$, Berlin, Germany). System control and data acquisition were conducted using the included Prep SFC software.

Reverse-phase purification was performed using an Agilent 1200 HPLC-MSD system consisting of a 6130B single quadrupole mass-selective detector (MSD), G1315B diode array detector (DAD), G2258A autosampler, two G1361A preparative pumps, one G1379A quaternary pump with degasser, one G1312A binary pump, and three G1364B fraction collectors from Agilent Technologies (Agilent Technologies, Palo Alto, CA). System control and data analysis were performed using Agilent's ChemStation software, revision B.04.03. A Waters SunFire C18 OBD Prep Column, 100A, $5 \mu \mathrm{m}, 19 \mathrm{~mm}$ X $150 \mathrm{~mm}$ column was used as the stationary phase (Waters Corporation, Milford, MA, USA). Gradient elution was carried out using water (solvent A) and acetonitrile (solvent B) as a mobile phase. Either a 10\% trifluoroacetic acid solution or ammonium hydroxide solution was teed into the mobile phase as a modifier using a static mixer prior to the column, pumped at $1 \%$ of the total mobile phase flowrate. Electrospray (ESI) Mass-triggered fraction collected was employed using positive ion polarity scanning to monitor for the target mass. 


\section{Experimental Information}

\section{Starting Material Synthesis}

The dehydroproline starting materials $\mathbf{1}, \mathbf{S - 1}, \mathbf{S - 2}$, and $\mathbf{S - 3}$ were prepared according to the literature. ${ }^{1}$
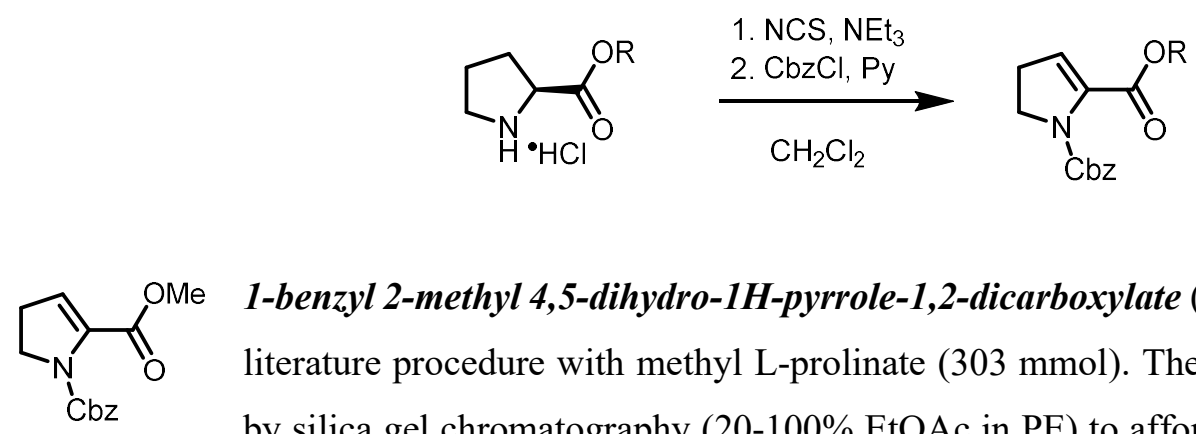

1-benzyl 2-methyl 4,5-dihydro-1H-pyrrole-1,2-dicarboxylate (1). Prepared according to the literature procedure with methyl L-prolinate $(303 \mathrm{mmol})$. The crude material was purified by silica gel chromatography (20-100\% EtOAc in PE) to afford the title compound as pale yellow oil (51.3 g, 65\% yield). All spectra were in accord with the literature. ${ }^{1}$

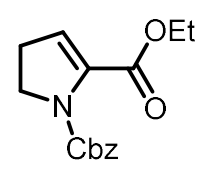

1-benzyl 2-ethyl 4,5-dihydro-1H-pyrrole-1,2-dicarboxylate (S-1). Prepared according to the literature precedent with ethyl L-prolinate $(6.0 \mathrm{mmol})$. The crude material was purified by silica gel chromatography (0-45\% EtOAc in hexanes) to afford the title compound as pale yellow oil ( $0.16 \mathrm{~g}, 10 \%$ yield). All spectra were in accord with the literature. ${ }^{2}$

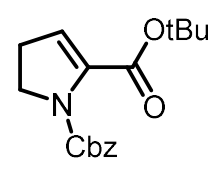

1-benzyl 2-(tert-butyl) 4,5-dihydro-1H-pyrrole-1,2-dicarboxylate (S-2). Prepared according to the literature precedent with tert-butyl L-prolinate $(5.8 \mathrm{mmol})$. The crude material was purified by silica gel chromatography (0-45\% EtOAc in hexanes) to afford the title compound as pale yellow oil $(0.91 \mathrm{~g}, 51 \%$ yield $)$. All spectra were in accord with the literature. ${ }^{3}$

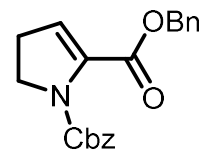

dibenzyl 4,5-dihydro-1H-pyrrole-1,2-dicarboxylate (S-3). Prepared according to the literature precedent with benzyl L-prolinate $(6.0 \mathrm{mmol})$. The crude material was purified by silica gel chromatography $(0-45 \%$ EtOAc in hexanes) to afford the title compound as pale yellow oil (1.3 g, 66\% yield). ${ }^{1} \mathbf{H}$ NMR (600 MHz, $\left.\mathrm{CDCl}_{3}\right) \delta 7.46-7.28(\mathrm{~m}, 10 \mathrm{H}), 5.89(\mathrm{t}, J=2.9 \mathrm{~Hz}, 1 \mathrm{H})$, $5.14(\mathrm{~s}, 2 \mathrm{H}), 5.12(\mathrm{~s}, 2 \mathrm{H}), 4.02(\mathrm{t}, J=8.9 \mathrm{~Hz}, 2 \mathrm{H}), 2.68(\mathrm{td}, J=9.0,2.9 \mathrm{~Hz}, 2 \mathrm{H}) .{ }^{13} \mathbf{C} \mathbf{~ N M R}(151 \mathrm{MHz}$, $\left.\mathrm{CDCl}_{3}\right) \delta 161.9,153.6,136.4,136.1,135.5,128.6,128.44,128.37,128.27,128.25,120.5,67.8,67.0,48.6$, 28.8. HRESIMS $360.1205 \mathrm{~m} / \mathrm{z}$ (calc'd for $\mathrm{C}_{20} \mathrm{H}_{19} \mathrm{NO}_{4} \mathrm{Na}^{+}, \mathrm{m} / \mathrm{z} 360.1212$; $\Delta \mathrm{ppm} 1.9$ ) 

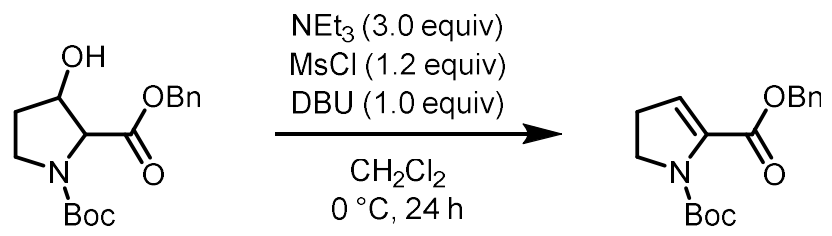

S-4

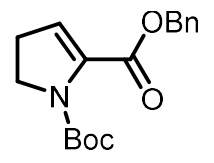

2-benzyl 1-(tert-butyl) 4,5-dihydro-1H-pyrrole-1,2-dicarboxylate (S-4). To a solution of 2benzyl 1-(tert-butyl) 3-hydroxypyrrolidine-1,2-dicarboxylate (40.0 g, $125 \mathrm{mmol})$ in $\mathrm{CH}_{2} \mathrm{Cl}_{2}$ (400 mL) were added successively $\mathrm{NEt}_{3}(37.8 \mathrm{~g}, 374 \mathrm{mmol}, 3.0$ equiv) and $\mathrm{MsCl}(17.1 \mathrm{~g}$, $150 \mathrm{mmol}, 1.2$ equiv) at $0{ }^{\circ} \mathrm{C}$. After stirring for $2 \mathrm{~h}$ at $0{ }^{\circ} \mathrm{C}, \mathrm{LCMS}$ showed that $\mathrm{SM}$ was converted into the intermediate. DBU (8.90 g, $125.0 \mathrm{mmol}, 1.0$ equiv) was then added and the mixture was left to stir for 24 $\mathrm{h}$ at $0{ }^{\circ} \mathrm{C}$. Water $(400 \mathrm{~mL})$ was added, the mixture was allowed to warm to $\mathrm{rt}$, and the aqueous layer was extracted three times with $\mathrm{CH}_{2} \mathrm{Cl}_{2}(400 \mathrm{ml})$. The combined organic phase was washed with brine, dried over $\mathrm{Na}_{2} \mathrm{SO}_{4}$ and concentrated under reduced pressure. The crude mixture was purified by silica gel chromatography (20-100\% EtOAc in PE) to afford the title compound as yellow oil ( $30.9 \mathrm{~g}, 82 \%$ yield). ${ }^{1}$ H NMR (400 MHz, Chloroform- $d$ ) $\delta 7.46-7.39$ (m, 2H), $7.43-7.30(\mathrm{~m}, 3 \mathrm{H}), 5.84(\mathrm{t}, J=3.0 \mathrm{~Hz}, 1 \mathrm{H})$, 5.26 (s, 2H), 3.95 (dd, $J=9.4,8.5 \mathrm{~Hz}, 2 \mathrm{H}), 2.65$ (ddd, $J=9.5,8.5,3.0 \mathrm{~Hz}, 2 \mathrm{H}), 1.46$ (s, 9H). ${ }^{13} \mathbf{C} \mathbf{~ N M R}$ $\left(151 \mathrm{MHz}, \mathrm{CDCl}_{3}\right) \delta 162.2,153.0,136.9,135.6,128.7,128.42,128.40,120.0,81.2,67.0,48.5,28.7,28.2$.

HRESIMS $326.1368 \mathrm{~m} / \mathrm{z}$ (calc'd for $\mathrm{C}_{17} \mathrm{H}_{21} \mathrm{NO}_{4} \mathrm{Na}^{+}, \mathrm{m} / \mathrm{z} 326.1368 \mathrm{~m} / \mathrm{z} ; \Delta \mathrm{ppm} 0.0$ )

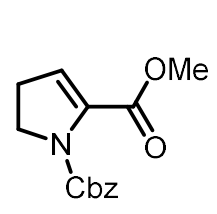

1

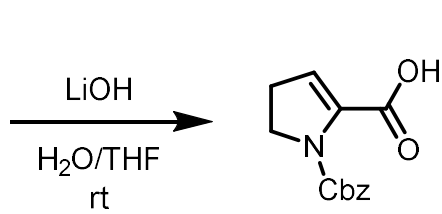

S-5

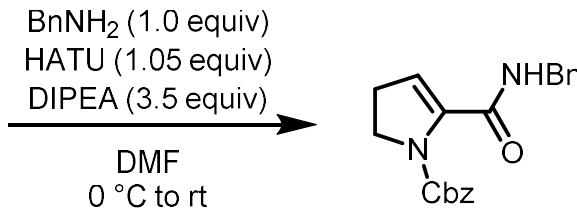

S-6

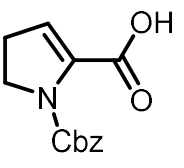

1-((benzyloxy)carbonyl)-4,5-dihydro-1H-pyrrole-2-carboxylic acid (S-5). Prepared according to the literature procedure with $\mathbf{1}(7.65 \mathrm{mmol})$ to afford the title compound as offwhite solid (1.59 g, 84\% yield). All spectra were in accord with the literature. ${ }^{1}$

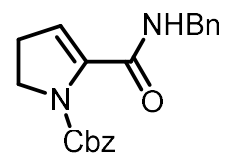

benzyl 5-(benzylcarbamoyl)-2,3-dihydro-1H-pyrrole-1-carboxylate (S-6). To a 40-mL vial equipped with a stir bar was added $\mathbf{S - 5}(508 \mathrm{mg}, 2.05 \mathrm{mmol}, 1.1$ equiv). The vial was purged with $\mathrm{N}_{2}$, then DMF $(9.4 \mathrm{~mL})$ and DIPEA (1.14 mL, $6.53 \mathrm{mmol}, 3.5$ equiv) was added. The vial was cooled to $0{ }^{\circ} \mathrm{C}$ in an ice bath. HATU (745 mg, $1.96 \mathrm{mmol}, 1.05$ equiv) was added in one portion followed by benzylamine $(0.204 \mathrm{~mL}, 1.87 \mathrm{mmol}, 1.0$ equiv) dropwise. The ice batch was removed, and the reaction mixture was allowed to warm to $\mathrm{rt}$ and left to stir for $2 \mathrm{~h}$. The reaction mixture 
was then added dropwise into $\mathrm{H}_{2} \mathrm{O}(20.0 \mathrm{~mL})$ while stirring. White solid precipitated from solution which was collected by filtration to afford the title compound as white solid (504 mg, 80\% yield). ${ }^{1} \mathbf{H}$ NMR (600 MHz, DMSO- $\left.d_{6}\right) \delta 8.67(\mathrm{t}, J=6.0 \mathrm{~Hz}, 1 \mathrm{H}), 7.38-7.20(\mathrm{~m}, 10 \mathrm{H}), 5.61(\mathrm{t}, J=2.8 \mathrm{~Hz}, 1 \mathrm{H}), 5.05(\mathrm{~s}, 2 \mathrm{H}), 4.27$ $(\mathrm{d}, J=6.0 \mathrm{~Hz}, 2 \mathrm{H}), 3.87(\mathrm{t}, J=8.9 \mathrm{~Hz}, 2 \mathrm{H}), 2.58(\mathrm{td}, J=9.0,2.7 \mathrm{~Hz}, 2 \mathrm{H}) .{ }^{13} \mathbf{C}$ NMR $(151 \mathrm{MHz}, \mathrm{DMSO}-$ $\left.d_{6}\right) \delta 161.5,152.5,139.2,136.5,128.3,128.1,127.8,127.5,127.2,126.7,115.7,66.5,48.3,41.9,40.1$, 27.8. HRESIMS $337.1548 \mathrm{~m} / \mathrm{z}$ (calc'd for $\mathrm{C}_{20} \mathrm{H}_{21} \mathrm{~N}_{2} \mathrm{O}_{3}{ }^{+}, \mathrm{m} / \mathrm{z} 337.1552 \mathrm{~m} / \mathrm{z} \mathrm{m} / \mathrm{z} ; \Delta \mathrm{ppm} 1.9$ ).

\section{Preliminary results and chiral ligand screen}

Procedure for reactions in Table 1

In a $\mathrm{N}_{2}$-filled glove box a 2-dram vial equipped with a stir bar was charged with the rhodium complex $(15 \mu \mathrm{mol}, 5.0 \mathrm{~mol} \%$ for the monomeric Rh complex, or $7.5 \mu \mathrm{mol}, 2.5 \mathrm{~mol} \%$ for dimeric Rh complexes), ligand (if used, $16 \mu \mathrm{mol}, 5.25 \mathrm{~mol} \%)$, and toluene $(0.50 \mathrm{~mL})$ and the mixture was left to stir for 10 minutes. 1 (78 mg, $0.30 \mathrm{mmol}, 1.0$ equiv) and phenylboronic acid (44 mg, $0.36 \mathrm{mmol}, 1.2$ equiv) were added to the catalyst mixture together as a solution in the remaining toluene $(1.0 \mathrm{~mL}) . \mathrm{K}_{3} \mathrm{PO}_{4}(\mathrm{aq}, 0.30$ $\mathrm{mL}, 4 \mathrm{M}$ solution, $1.2 \mathrm{mmol}, 4.0$ equiv. Solution had been previously prepared, sparged with and stored under $\mathrm{N}_{2}$ ) was added. The vial was sealed with a cap containing Teflon septa, removed from the glove box, and was placed in an aluminum block heated to $60{ }^{\circ} \mathrm{C}$ on top of a stir plate and left to stir for $15 \mathrm{~h}$. The reaction mixture was allowed to cool to room temperature. $3.0 \mu \mathrm{L}$ of the mixture was added to $1.0 \mathrm{~mL}$ of 10:1 MeCN: $\mathrm{H}_{2} \mathrm{O}$ and ran on UPLC to determine LCAP (toluene peak not integrated). $10 \mu \mathrm{L}$ of the mixture of the mixture was then concentrated under reduced pressure and ran on SFC-MS to determine dr and ee. 
Table S-1. Table 1 with added remaining starting material LCAP numbers. The absolute value of the ee is reported (leel).

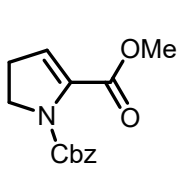

1

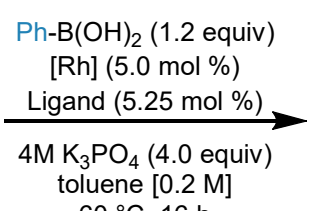

$60{ }^{\circ} \mathrm{C}, 16 \mathrm{~h}$

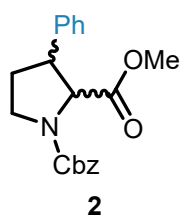

2

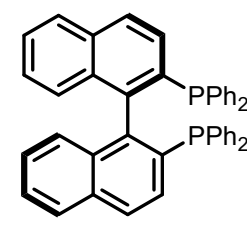

L1

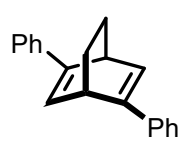

L2

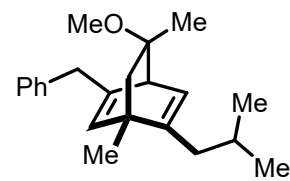

L3

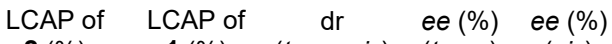

\begin{tabular}{cccccccc} 
entry & {$[\mathrm{Rh}]$} & Ligand & $\begin{array}{c}\text { LCAP of } \\
\mathbf{2}(\%)\end{array}$ & $\begin{array}{c}\text { LCAP of } \\
\mathbf{1}(\%)\end{array}$ & $\begin{array}{c}\mathrm{dr} \\
\text { (trans:cis) }\end{array}$ & $\begin{array}{c}\text { ee }(\%) \\
\text { (trans) }\end{array}$ & $\begin{array}{c}\text { ee }(\%) \\
\text { (cis) }\end{array}$ \\
\hline 1 & $\mathrm{Rh}(\mathrm{nbd})_{2} \mathrm{BF}_{4}$ & - & 94 & 6 & $14: 1$ & - & - \\
2 & $\mathrm{Rh}(\mathrm{nbd})_{2} \mathrm{BF}_{4}$ & $\mathbf{L 1}$ & 52 & 28 & $1: 1$ & 66 & 96 \\
3 & {$\left[\mathrm{Rh}\left(\mathrm{C}_{2} \mathrm{H}_{4}\right)_{2} \mathrm{Cl}\right]_{2}$} & - & $<5$ & 78 & - & - & - \\
4 & {$\left[\mathrm{Rh}\left(\mathrm{C}_{2} \mathrm{H}_{4}\right)_{2} \mathrm{Cl}\right]_{2}$} & $\mathbf{L 1}$ & 54 & 22 & $1: 1$ & 69 & 96 \\
5 & {$\left[\mathrm{Rh}\left(\mathrm{C}_{2} \mathrm{H}_{4}\right)_{2} \mathrm{Cl}\right]_{2}$} & L2 & 78 & 15 & $2.2: 1$ & 89 & 91 \\
6 & {$\left[\mathrm{Rh}\left(\mathrm{C}_{2} \mathrm{H}_{4}\right)_{2} \mathrm{Cl}\right]_{2}$} & L3 & 17 & 73 & $3: 1$ & 47 & nd \\
\hline
\end{tabular}

Reactions were performed on $0.3 \mathrm{mmol}$ scale. Liquid chromatography area percent (LCAP) determined by UPLC. dr and ee determined by SFC.

\section{Procedure for chiral ligand screen}

In a $\mathrm{N}_{2}$-filled glove box, a 96-well reaction block with $1 \mathrm{~mL}$ shell vials containing a stir bar and a different chiral ligand $(0.420 \mu \mathrm{mol}, 5.25 \mathrm{~mol} \%)$ was charged with $\mathrm{Rh}(\mathrm{nbd})_{2} \mathrm{BF}_{4}(0.150 \mathrm{mg}, 0.400 \mu \mathrm{mol}$, $5.00 \mathrm{~mol} \%)$ as a solution in toluene $(40.0 \mu \mathrm{L})$. The block was sealed and stirred on a tumble stirrer at room temperature for 10 minutes inside the glove box. The block was then opened, and 1 ( $2.09 \mathrm{mg}, 8.00 \mu \mathrm{mol}$, 1.00 equiv) and phenylboronic acid ( $1.17 \mathrm{mg}, 9.60 \mu \mathrm{mol}, 1.20$ equiv) were added together to each vial as a solution in toluene $(40.0 \mu \mathrm{L}) . \mathrm{K}_{3} \mathrm{PO}_{4}(\mathrm{aq}, 4.0 \mathrm{M}, 8.0 \mu \mathrm{L}, 32 \mu \mathrm{mol}, 4.0$ equiv. had been previously prepared, sparged with and stored under $\mathrm{N}_{2}$ ) was added to each vial. The block was sealed and was left to stir on a tumble stirrer inside the glove box at $60{ }^{\circ} \mathrm{C}$ for $17 \mathrm{~h}$. The block was removed from the tumble stirrer, allowed to cool to room temperature, and removed from the glove box. The block was opened and $400 \mu \mathrm{L}$ of quench solution containing internal standard (2.0 mM biphenyl in 3:1 $\left.\mathrm{MeCN}: \mathrm{H}_{2} \mathrm{O}\right)$ was added. The block was sealed again and stirred on the tumble stirrer for 15 minutes. The block was then removed from the tumble stirrer and allowed to settle. The block was opened and SFC samples were prepared by diluting 50 $\mu \mathrm{L}$ of the quenched reaction mixtures in $250 \mu \mathrm{L}$ of $\mathrm{MeCN}$ in a 96-well $2 \mathrm{~mL}$ plate that was run directly on the SFC (Lux-4 column, MeOH modifier) to determine yield, diastereomeric excess (de) and enantiomeric 
excess (ee). The yields are shown as the ratio of product to internal standard and represented as a percentage normalized to the reaction with Ph-BPE (L7, 94\% by SFC area percent). The absolute value of the ee is reported (leel).

Figure S-1. Detailed summary of ligand screen

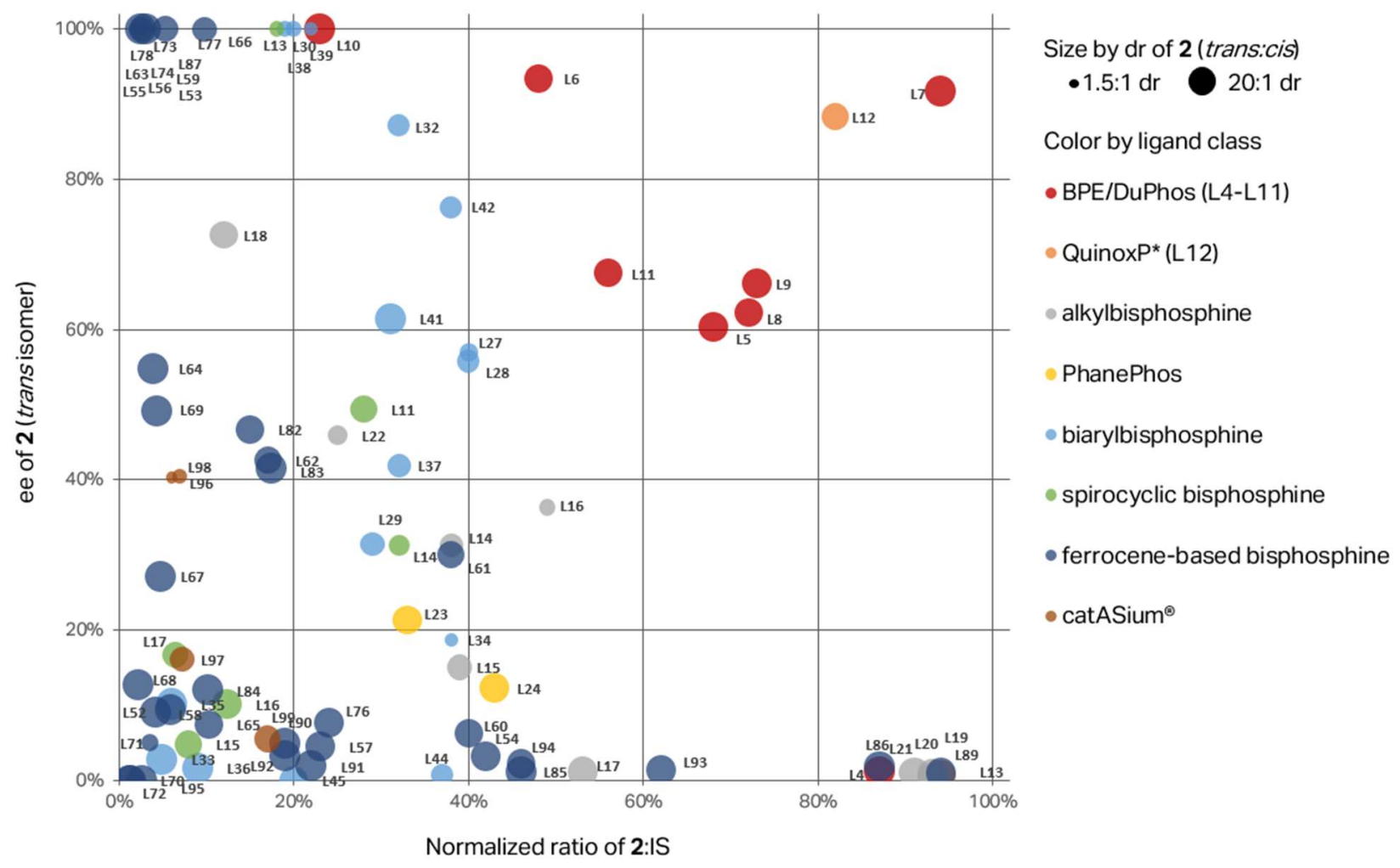

\begin{tabular}{|c|c|c|c|c|c|}
\hline Ligand \# & Normalized 2:IS & de & ee & Ligand Name & Ligand Class \\
\hline L4 & $87 \%$ & $93 \%$ & $1 \%$ & Me-BPE & BPE/DuPhos \\
\hline L5 & $68 \%$ & $94 \%$ & $60 \%$ & Et-BPE & BPE/DuPhos \\
\hline L6 & $48 \%$ & $90 \%$ & $93 \%$ & iPr-BPE & BPE/DuPhos \\
\hline L7 & $94 \%$ & $95 \%$ & $92 \%$ & Ph-BPE & BPE/DuPhos \\
\hline L8 & $72 \%$ & $86 \%$ & $62 \%$ & Me-Duphos & BPE/DuPhos \\
\hline L9 & $73 \%$ & $90 \%$ & $66 \%$ & Et-Duphos & BPE/DuPhos \\
\hline L10 & $23 \%$ & $100 \%$ & $100 \%$ & iPr-Duphos & BPE/DuPhos \\
\hline L12 & $82 \%$ & $77 \%$ & $88 \%$ & QuinoxP* & QuinoxP* \\
\hline L13 & $94 \%$ & $94 \%$ & $1 \%$ & BenzP* & QuinoxP \\
\hline L11 & $56 \%$ & $82 \%$ & $68 \%$ & Me-UCAP & BPE/DuPhos \\
\hline L14 & $38 \%$ & $59 \%$ & $31 \%$ & Nap-DIPAMP & alkylbisphosphine \\
\hline L15 & $39 \%$ & $66 \%$ & $15 \%$ & tol-DIPAMP & alkylbisphosphine \\
\hline L16 & $49 \%$ & $31 \%$ & $36 \%$ & iPr-DIPAMP $^{*}$ & alkylbisphosphine \\
\hline L17 & $53 \%$ & $93 \%$ & $1 \%$ & Ph $_{2}$-DIPAMP & alkylbisphosphine \\
\hline L18 & $12 \%$ & $80 \%$ & $73 \%$ & ProPhos & alkylbisphosphine \\
\hline L19 & $93 \%$ & $93 \%$ & $0 \%$ & BisP* 2HBF $_{4}$ & alkylbisphosphine \\
\hline
\end{tabular}




\begin{tabular}{|c|c|c|c|c|c|}
\hline L20 & $93 \%$ & $92 \%$ & $1 \%$ & miniPHOS & alkylbisphosphine \\
\hline L21 & $91 \%$ & $95 \%$ & $1 \%$ & TCFP $2 \mathrm{HBF}_{4}$ & alkylbisphosphine \\
\hline L22 & $25 \%$ & $44 \%$ & $46 \%$ & MaxPhos & alkylbisphosphine \\
\hline L23 & $33 \%$ & $86 \%$ & $21 \%$ & PhanePhos & PhanePhos \\
\hline L24 & $43 \%$ & $91 \%$ & $12 \%$ & An-PhanePhos & PhanePhos \\
\hline $\mathrm{L} 25$ & $0 \%$ & - & - & MP²-Segphos $^{2}$ & biarylbisphosphine \\
\hline L26 & $0 \%$ & - & - & $\mathrm{P}^{3}$-Segphos & biarylbisphosphine \\
\hline L27 & $40 \%$ & $34 \%$ & $57 \%$ & Cy-Segphos & biarylbisphosphine \\
\hline L28 & $40 \%$ & $53 \%$ & $56 \%$ & DMM-Segphos & biarylbisphosphine \\
\hline L29 & $29 \%$ & $58 \%$ & $32 \%$ & synPhos & biarylbisphosphine \\
\hline L30 & $19 \%$ & $28 \%$ & $100 \%$ & $\begin{array}{l}\text { tol-MeO- } \\
\text { BIPHEP }\end{array}$ & biarylbisphosphine \\
\hline L31 & $0 \%$ & - & - & $\begin{array}{l}\text { iPr-Me }{ }_{2} \mathrm{NPh}- \\
\mathrm{MeO}-\mathrm{BIPHEP}\end{array}$ & biarylbisphosphine \\
\hline L32 & $32 \%$ & $50 \%$ & $87 \%$ & $\begin{array}{l}\text { 3,4,5-MeOPh- } \\
\text { MeO-BIPHEP }\end{array}$ & biarylbisphosphine \\
\hline L33 & $5 \%$ & $100 \%$ & $3 \%$ & $\begin{array}{c}\text { 3,5-tBuPh- } \\
\text { MeO-BIPHEP }\end{array}$ & biarylbisphosphine \\
\hline L34 & $38 \%$ & $21 \%$ & $19 \%$ & $\begin{array}{l}\text { fur-OMe- } \\
\text { BIPHEP }\end{array}$ & biarylbisphosphine \\
\hline L35 & $6 \%$ & $100 \%$ & $10 \%$ & $\begin{array}{l}\text { iPr-MeO- } \\
\text { BIPHEP }\end{array}$ & biarylbisphosphine \\
\hline L36 & $9 \%$ & $100 \%$ & $2 \%$ & $\begin{array}{c}\text { cycloBu-MeO- } \\
\text { BIPHEP }\end{array}$ & biarylbisphosphine \\
\hline L37 & $32 \%$ & $58 \%$ & $42 \%$ & MeO-BIPHEP & biarylbisphosphine \\
\hline L38 & $22 \%$ & $20 \%$ & $100 \%$ & Ph-Garphos & biarylbisphosphine \\
\hline L39 & $20 \%$ & $29 \%$ & $100 \%$ & tol-Garphos & biarylbisphosphine \\
\hline L40 & $0 \%$ & - & - & xyl-Garphos & biarylbisphosphine \\
\hline L41 & $31 \%$ & $100 \%$ & $61 \%$ & $\begin{array}{l}\text { BTFM- } \\
\text { Garphos }\end{array}$ & biarylbisphosphine \\
\hline L42 & $38 \%$ & $52 \%$ & $76 \%$ & DMM-Garphos & biarylbisphosphine \\
\hline L43 & $0 \%$ & - & - & $\begin{array}{l}\text { DTBM- } \\
\text { Garphos }\end{array}$ & biarylbisphosphine \\
\hline L44 & $37 \%$ & $52 \%$ & $1 \%$ & H8-Binam-P & biarylbisphosphine \\
\hline L45 & $20 \%$ & $84 \%$ & $0 \%$ & Binam-P & biarylbisphosphine \\
\hline L46 & $18 \%$ & $24 \%$ & $100 \%$ & SDP & spirocyclic bisphosphine \\
\hline L47 & $28 \%$ & $75 \%$ & $49 \%$ & xyl-SDP & spirocyclic bisphosphine \\
\hline L48 & $32 \%$ & $45 \%$ & $31 \%$ & tol-SDP & spirocyclic bisphosphine \\
\hline L49 & $8 \%$ & $79 \%$ & $5 \%$ & Ph-SKP & spirocyclic bisphosphine \\
\hline L50 & $12 \%$ & $89 \%$ & $10 \%$ & tol-SKP & spirocyclic bisphosphine \\
\hline L51 & $6 \%$ & $70 \%$ & $17 \%$ & xyl-SKP & spirocyclic bisphosphine \\
\hline L52 & $4 \%$ & $100 \%$ & $9 \%$ & Me-Ferrolane & ferrocene-based bisphosphine \\
\hline L53 & $2 \%$ & $6 \%$ & $100 \%$ & Et-Ferrolane & ferrocene-based bisphosphine \\
\hline L54 & $42 \%$ & $92 \%$ & $3 \%$ & iPr-Ferrolane & ferrocene-based bisphosphine \\
\hline L55 & $2 \%$ & $12 \%$ & $100 \%$ & Et-FerroTane & ferrocene-based bisphosphine \\
\hline L56 & $3 \%$ & $64 \%$ & $100 \%$ & Trifer & ferrocene-based bisphosphine \\
\hline L57 & $23 \%$ & $94 \%$ & $5 \%$ & Chenphos & ferrocene-based bisphosphine \\
\hline L58 & $6 \%$ & $100 \%$ & $10 \%$ & tBu-PHO-Fc & ferrocene-based bisphosphine \\
\hline L59 & $3 \%$ & $20 \%$ & $100 \%$ & PhPHO-Fc & ferrocene-based bisphosphine \\
\hline
\end{tabular}




\begin{tabular}{|c|c|c|c|c|c|}
\hline L60 & $40 \%$ & $88 \%$ & $6 \%$ & $\begin{array}{l}\text { RRRMe- } \\
\text { Kephos }\end{array}$ & ferrocene-based bisphosphine \\
\hline L61 & $38 \%$ & $79 \%$ & $30 \%$ & $\begin{array}{l}\text { SSRMe- } \\
\text { Kephos }\end{array}$ & ferrocene-based bisphosphine \\
\hline L62 & $17 \%$ & $79 \%$ & $43 \%$ & SL-J005-1 & ferrocene-based bisphosphine \\
\hline L63 & $2 \%$ & $3 \%$ & $100 \%$ & SL-J014-1 & ferrocene-based bisphosphine \\
\hline L64 & $4 \%$ & $100 \%$ & $55 \%$ & SL-J013-1 & ferrocene-based bisphosphine \\
\hline L65 & $10 \%$ & $83 \%$ & $7 \%$ & SL-J211-1 & ferrocene-based bisphosphine \\
\hline L66 & $10 \%$ & $63 \%$ & $100 \%$ & SL-J213-1 & ferrocene-based bisphosphine \\
\hline L67 & $5 \%$ & $100 \%$ & $27 \%$ & SL-J216-1 & ferrocene-based bisphosphine \\
\hline L68 & $2 \%$ & $100 \%$ & $13 \%$ & SL-J219-1 & ferrocene-based bisphosphine \\
\hline L69 & $4 \%$ & $100 \%$ & $49 \%$ & SL-J220-1 & ferrocene-based bisphosphine \\
\hline L70 & $1 \%$ & $100 \%$ & - & SL-J221-1 & ferrocene-based bisphosphine \\
\hline L71 & $4 \%$ & $32 \%$ & $5 \%$ & SL-J222-1 & ferrocene-based bisphosphine \\
\hline L72 & $1 \%$ & $100 \%$ & - & SL-J305-1 & ferrocene-based bisphosphine \\
\hline L73 & $2 \%$ & $17 \%$ & $100 \%$ & SL-J404-1 & ferrocene-based bisphosphine \\
\hline L74 & $2 \%$ & $18 \%$ & $100 \%$ & SL-J409-1 & ferrocene-based bisphosphine \\
\hline $\mathrm{L75}$ & $0 \%$ & - & - & SL-J502-1 & ferrocene-based bisphosphine \\
\hline L76 & $24 \%$ & $88 \%$ & $8 \%$ & SL-J505-1 & ferrocene-based bisphosphine \\
\hline L77 & $5 \%$ & $70 \%$ & $100 \%$ & SL-J506-1 & ferrocene-based bisphosphine \\
\hline L78 & $2 \%$ & $100 \%$ & $100 \%$ & SL-J215-2 & ferrocene-based bisphosphine \\
\hline L79 & $0 \%$ & - & - & SL-J302-1 & ferrocene-based bisphosphine \\
\hline L80 & $0 \%$ & - & - & SL-J011-1 & ferrocene-based bisphosphine \\
\hline L81 & $0 \%$ & - & - & SL-J304-1 & ferrocene-based bisphosphine \\
\hline L82 & $15 \%$ & $82 \%$ & $47 \%$ & SL-J408-1 & ferrocene-based bisphosphine \\
\hline L83 & $17 \%$ & $100 \%$ & $42 \%$ & SL-J411-2 & ferrocene-based bisphosphine \\
\hline L84 & $10 \%$ & $100 \%$ & $12 \%$ & SL-J412-1 & ferrocene-based bisphosphine \\
\hline L85 & $46 \%$ & $93 \%$ & $1 \%$ & $\begin{array}{c}\text { SL-J851-2 (bis } \\
\text { J001) }\end{array}$ & ferrocene-based bisphosphine \\
\hline L86 & $87 \%$ & $94 \%$ & $2 \%$ & $\begin{array}{c}\text { SL-J852-2 (bis } \\
\text { J005) }\end{array}$ & ferrocene-based bisphosphine \\
\hline L87 & $3 \%$ & $100 \%$ & $100 \%$ & $\begin{array}{c}\text { SL-J853-2 (bis } \\
\text { J013) }\end{array}$ & ferrocene-based bisphosphine \\
\hline L88 & $0 \%$ & - & - & Me-TwinPhos & ferrocene-based bisphosphine \\
\hline L89 & $94 \%$ & $94 \%$ & $1 \%$ & Cy-TwinPhos & ferrocene-based bisphosphine \\
\hline L90 & $19 \%$ & $100 \%$ & $5 \%$ & SL-W022-1 & ferrocene-based bisphosphine \\
\hline L91 & $22 \%$ & $100 \%$ & $2 \%$ & SL-W016-1 & ferrocene-based bisphosphine \\
\hline L92 & $19 \%$ & $100 \%$ & $3 \%$ & SL-W023-1 & ferrocene-based bisphosphine \\
\hline L93 & $62 \%$ & $94 \%$ & $1 \%$ & SL-W012-1 & ferrocene-based bisphosphine \\
\hline L94 & $46 \%$ & $88 \%$ & $2 \%$ & SL-W017-1 & ferrocene-based bisphosphine \\
\hline L95 & $3 \%$ & $100 \%$ & - & $\begin{array}{c}\text { CTH- } \\
\text { JAFAphos }\end{array}$ & ferrocene-based bisphosphine \\
\hline L96 & $6 \%$ & $13 \%$ & $40 \%$ & catASium ${ }^{\circledR} T 1$ & catASium ${ }^{\circledR}$ \\
\hline L97 & $7 \%$ & $69 \%$ & $16 \%$ & catASium $®$ T2 & catASium ${ }^{\circledR}$ \\
\hline L98 & $7 \%$ & $24 \%$ & $40 \%$ & catASium $®$ T3 & catASium $\AA$ \\
\hline L99 & $17 \%$ & $71 \%$ & $6 \%$ & catASium $® I$ & catASium $\AA$ \\
\hline
\end{tabular}




\section{General procedures}

Note: $2 \mathrm{M} \mathrm{K}_{3} \mathrm{PO}_{4}$ was prepared in a $20 \mathrm{~mL}$ volumetric flask with HPLC-grade water and sparged with $\mathrm{N}_{2}$ for 10 minutes before being stored in a $40 \mathrm{~mL}$ vial with a cap containing teflon septa under $\mathrm{N}_{2}$. This solution was stored and used for several months after preparation.

\section{General Procedure A - glove box procedure:}

Performed on $0.50 \mathrm{mmol}$ scale unless otherwise stated. In a $\mathrm{N}_{2}$-filled glove box, a 2-dram vial equipped with a stir bar was charged with $(R, R)$-Ph-BPE-Rh $(8.1 \mathrm{mg}, 10 \mu \mathrm{mol}, 2.0 \mathrm{~mol} \%), 1$ (130 mg, 0.50 mmol, 1.0 equiv), the boronic acid, ester, or potassium trifluoroborate salt (1.2 - 1.5 equiv), and 2-MeTHF $(2.5 \mathrm{~mL}, 0.20 \mathrm{M})$. The vial was sealed with a cap containing Teflon septa and removed from the glove box. Under an inlet needle of $\mathrm{N}_{2}, \mathrm{~K}_{3} \mathrm{PO}_{4}\left(0.50 \mathrm{~mL}, 2.0 \mathrm{M}\right.$ solution, $1.0 \mathrm{mmol}, 2.0$ equiv) was added. The $\mathrm{N}_{2}$ inlet was removed and the vial was placed in an aluminum block heated to $60{ }^{\circ} \mathrm{C}$ on top of a stir plate and left to stir for $16 \mathrm{~h}$. The reaction mixture was allowed to cool to room temperature and diluted with EtOAc and a small amount of water. The layers were separated, and the aqueous layer was extracted an additional two times with EtOAc. The combined organics were dried over $\mathrm{MgSO}_{4}$, filtered, and concentrated under reduced pressure. The crude material was analyzed by ${ }^{1} \mathrm{H}$ NMR spectroscopy to determine the diastereomeric ratio then the crude material was purified on silica gel eluting with ethyl acetate in hexanes. The purified material was analyzed by SFC-MS to determine the enantiomeric excess.

General Procedure B - glove box free procedure:

A 2-dram vial equipped with a stir bar was charged with $(R, R)$-Ph-BPE-Rh $(8.1 \mathrm{mg}, 10 \mu \mathrm{mol}, 2.0$ mol \%), 1 (130 mg, $0.50 \mathrm{mmol}, 1.0$ equiv), and the boronic acid, ester, or potassium trifluoroborate salt (1.2 - 1.5 equiv). The vial was sealed with a cap containing Teflon septa and evacuated and back-filled three times with $\mathrm{N}_{2}$. 2-MeTHF $(2.5 \mathrm{~mL}, 0.20 \mathrm{M})$ and $\mathrm{K}_{3} \mathrm{PO}_{4}(0.50 \mathrm{~mL}, 2.0 \mathrm{M}$ solution, $1.0 \mathrm{mmol}, 2.0$ equiv) were added. The $\mathrm{N}_{2}$ inlet was removed and the vial was placed in an aluminum block heated to $60{ }^{\circ} \mathrm{C}$ on top of a stir plate and left to stir for $16 \mathrm{~h}$. The reaction mixture was allowed to cool to room temperature and diluted with EtOAc and a small amount of water. The layers were separated, and the aqueous layer was extracted an additional two times with EtOAc. The combined organics were dried over $\mathrm{MgSO}_{4}$, filtered, and concentrated under reduced pressure. The crude material was analyzed by ${ }^{1} \mathrm{H}$ NMR spectroscopy to determine the diastereomeric ratio then the crude material was purified on silica gel eluting with ethyl acetate in hexanes. The purified material was analyzed by SFC-MS to determine the enantiomeric excess. 
General Procedure $C$ - with achiral ligand to prepare racemic mixtures of the reaction products:

In a $\mathrm{N}_{2}$-filled glove box, a 2-dram vial equipped with a stir bar was charged with $\mathrm{Rh}(\mathrm{nbd})_{2} \mathrm{BF}_{4}(5.6$ $\mathrm{mg}, 0.015 \mathrm{mmol}, 3.0 \mathrm{~mol} \%), 1(130 \mathrm{mg}, 0.50 \mathrm{mmol}, 1.0$ equiv), the boronic acid, ester, or potassium trifluoroborate salt $(1.2-1.5$ equiv), and dioxane $(2.5 \mathrm{~mL}, 0.20 \mathrm{M})$. The vial was sealed with a cap containing Teflon septa and removed from the glove box. Under an inlet needle of $\mathrm{N}_{2}, \mathrm{~K}_{3} \mathrm{PO}_{4}(0.50 \mathrm{~mL}$, 2.0 M solution, $1.0 \mathrm{mmol}, 2.0$ equiv) was added. The $\mathrm{N}_{2}$ inlet was removed and the vial was placed in an aluminum block heated to $60{ }^{\circ} \mathrm{C}$ on top of a stir plate and left to stir for $16 \mathrm{~h}$. The reaction mixture was allowed to cool to room temperature and diluted with EtOAc and a small amount of water. The layers were separated, and the aqueous layer was extracted an additional two times with EtOAc. The combined organics were dried over $\mathrm{MgSO}_{4}$, filtered, and concentrated under reduced pressure. The crude material was analyzed by ${ }^{1} \mathrm{H}$ NMR spectroscopy to determine the diastereomeric ratio then the crude material was purified on silica gel eluting with ethyl acetate in hexanes. The purified material was analyzed by SFC-MS to find separation conditions.

\section{General Procedure D-for pyridyl nucleophiles:}

Performed on $0.5 \mathrm{mmol}$ scale unless otherwise stated. In a $\mathrm{N}_{2}$-filled glove box, a 2-dram vial equipped with a stir bar was charged with $(R, R)$-Ph-BPE-Rh $(8.1 \mathrm{mg}, 10 \mu \mathrm{mol}, 2.0 \mathrm{~mol} \%), 1$ (130 mg, 0.50 mmol, 1.0 equiv), the pyridyl boronic acid (3.0 equiv), cesium carbonate (326 $\mathrm{mg}, 1.0 \mathrm{mmol}, 2.0$ equiv), and dioxane $(2.5 \mathrm{~mL}, 0.20 \mathrm{M})$. The vial was sealed with a cap containing Teflon septa and removed from the glove box. Under an inlet needle of $\mathrm{N}_{2}$, water (HPLC-grade) $(0.090 \mathrm{~mL}, 5.0 \mathrm{mmol}, 10$ equiv) was added. The $\mathrm{N}_{2}$ inlet was removed and the vial was placed in an aluminum block heated to $100{ }^{\circ} \mathrm{C}$ on top of a stir plate and left to stir for $16 \mathrm{~h}$. The reaction mixture was allowed to cool to room temperature and diluted with EtOAc and a small amount of water. The layers were separated, and the aqueous layer was extracted an additional two times with EtOAc. The combined organics were dried over $\mathrm{MgSO}_{4}$, filtered, and concentrated under reduced pressure. The crude material was analyzed by ${ }^{1} \mathrm{H}$ NMR spectroscopy to determine the diastereomeric ratio then the crude material was purified on silica gel eluting with ethyl acetate in hexanes. The purified material was analyzed by SFC-MS to determine the enantiomeric excess. 


\section{Reaction optimization}

Solvent/base screen:

In a $\mathrm{N}_{2}$-filled glove box, two 24-well reaction blocks with $1 \mathrm{~mL}$ shell vials each containing a stir bar were charged with $(R, R)$-Ph-BPE ( $0.24 \mathrm{mg}, 0.30 \mu \mathrm{mol}, 3.0 \mathrm{~mol} \%), 1$ (2.6 mg, $10 \mu \mathrm{mol}, 1.0$ equiv), and phenylboronic acid ( $1.5 \mathrm{mg}, 12 \mu \mathrm{mol}, 1.20$ equiv) together as a solution in the appropriate solvent (100 $\mu \mathrm{L}) . \mathrm{K}_{3} \mathrm{PO}_{4}, \mathrm{Cs}_{2} \mathrm{CO}_{3}$, and $\mathrm{K}_{2} \mathrm{CO}_{3}$ were then dosed as aqueous solutions (4.0 M, $10.0 \mu \mathrm{L}, 40 \mu \mathrm{mol}, 4.0$ equiv. the aq. solutions had been previously prepared and sparged with $\mathrm{N}_{2}$ ). Triethylamine (TEA) (5.6 $\mu \mathrm{L}, 40$ $\mu$ mol, 4.01 equiv) was added neat followed by $\mathrm{H}_{2} \mathrm{O}(4.4 \mu \mathrm{L})$. The reaction blocks were sealed, removed from the glove box, and were left to stir on a tumble stirrer at $60{ }^{\circ} \mathrm{C}$ for $16 \mathrm{~h}$. The block was removed from the tumble stirrer and allowed to cool to room temperature. The block was opened and $500 \mu \mathrm{L}$ of quench solution containing biphenyl as internal standard was added. $30 \mu \mathrm{L}$ of this solution was removed and diluted with $1.5 \mathrm{~mL}$ of $10: 1 \mathrm{MeCN}: \mathrm{H}_{2} \mathrm{O}$ then run on UPLC.

Figure S-2. Preliminary solvent/base screen

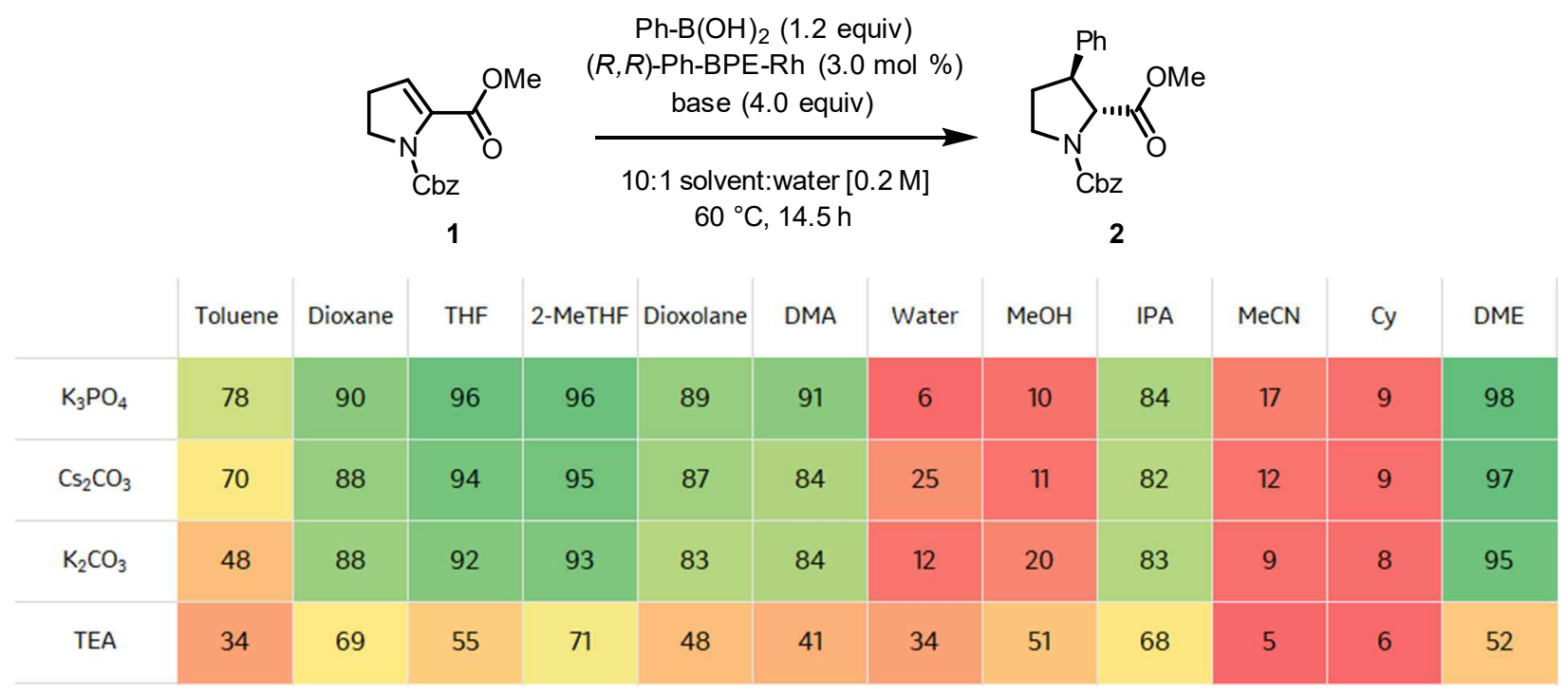

Numbers shown in heat map refer to ratio of $\mathbf{2}$ to remaining starting material $\mathbf{1}$ (represented as a percentage) determined by UPLC. 
Table S-2. Solvent Screen details. Reactions performed according to general procedure A

\begin{tabular}{|c|c|c|c|c|c|}
\hline \multirow[b]{3}{*}{ entry } & \multirow{3}{*}{$\begin{array}{l}1 \\
\text { solvent }\end{array}$} & \multirow{2}{*}{\multicolumn{2}{|c|}{$\begin{array}{c}\mathrm{Ph}-\mathrm{B}(\mathrm{OH})_{2}(1.2 \text { equiv }) \\
(R, R)-\mathrm{Ph}-\mathrm{BPE}^{\mathrm{Rh}}(3.0 \mathrm{~mol} \%) \\
4 \mathrm{M} \mathrm{K}_{3} \mathrm{PO}_{4}(4.0 \text { equiv }) \\
\text { solvent }(0.2 \mathrm{M}) \\
60^{\circ} \mathrm{C}, 14.5 \mathrm{~h}\end{array}$}} & \multirow{2}{*}{$\sum_{N_{\mathrm{Cbz}}^{\mathrm{Ph}}}^{\mathrm{Ph}}$} & \multirow[b]{3}{*}{$\begin{array}{l}\text { ee } \\
(\%)\end{array}$} \\
\hline & & & & & \\
\hline & & $\begin{array}{c}\mathrm{dr} \\
\text { (crude NMR) }\end{array}$ & $\begin{array}{c}\text { yield } \\
(\%)\end{array}$ & $\begin{array}{c}\text { recovered SM } \\
(\%)\end{array}$ & \\
\hline 1 & toluene & $19.4: 1$ & 78 & N/A & 94 \\
\hline 2 & dioxane & $18.4: 1$ & 74 & 5 & 94 \\
\hline 3 & THF & $26.5: 1$ & 78 & 1 & 96 \\
\hline 4 & 2-MeTHF & $26: 1$ & 78 & $\mathrm{~N} / \mathrm{A}$ & 97 \\
\hline 5 & dioxolane & $11.7: 1$ & 72 & 6 & 94 \\
\hline 6 & CPME & $26.4: 1$ & 79 & $\mathrm{~N} / \mathrm{A}$ & 96 \\
\hline 7 & DME & $19: 1$ & 78 & $\mathrm{~N} / \mathrm{A}$ & 95 \\
\hline
\end{tabular}

Reactions performed on $0.3 \mathrm{mmol}$ scale. dr determined by crude ${ }^{1} \mathrm{H}$ NMR. Yield of isolated material after column chromatography. ee determined by SFC-MS of material after purification

Table S-3. Conditions Screen. Reactions performed according to general procedure A.

\begin{tabular}{|c|c|c|c|c|c|c|c|c|c|}
\hline \multirow[b]{3}{*}{ entry } & \multirow[b]{3}{*}{$\begin{array}{l}\text { temp } \\
\left({ }^{\circ} \mathrm{C}\right)\end{array}$} & \multirow[b]{3}{*}{$\begin{array}{c}\text { time } \\
(h)\end{array}$} & \multirow{3}{*}{$\begin{array}{l}\mathrm{Cbz} \\
1 \\
\begin{array}{c}{[\mathrm{Rh}] \text { loading }} \\
(\mathrm{mol} \%)\end{array}\end{array}$} & \multirow{2}{*}{\multicolumn{2}{|c|}{$\begin{array}{c}\mathrm{Ph}-\mathrm{B}(\mathrm{OH})_{2}(1.2 \text { equiv }) \\
(R, R)-\mathrm{Ph}-\mathrm{BPE}-\mathrm{Rh} \\
\mathrm{K}_{3} \mathrm{PO}_{4}\end{array}$}} & \multirow{2}{*}{$\sum_{N}^{P}$} & \multirow{3}{*}{$\mathbb{O}_{\mathrm{O}}^{\mathrm{OMe}}$} & \multirow[b]{3}{*}{$\begin{array}{c}\text { recovered SM } \\
(\%)\end{array}$} & \multirow[b]{3}{*}{$\begin{array}{l}\mathrm{ee} \\
(\%)\end{array}$} \\
\hline & & & & & & & & & \\
\hline & & & & $\begin{array}{l}\mathrm{K}_{3} \mathrm{PO}_{4} \\
\text { (equiv) }\end{array}$ & solvent: $\mathrm{H}_{2} \mathrm{O}$ & $\begin{array}{c}\text { yield } \\
(\%)\end{array}$ & & & \\
\hline 1 & 60 & 14.5 & 3 & 4 & $5: 1$ & 78 & $19.4: 1$ & - & 94 \\
\hline 2 & 60 & $4 h$ & 2 & 2 & $10: 1$ & 76 & $18.8: 1$ & - & 96 \\
\hline 3 & 60 & $4 \mathrm{~h}$ & 2 & 2 & $5: 1$ & 83 & $21.2: 1$ & - & 96 \\
\hline 4 & 60 & $4 \mathrm{~h}$ & 2 & 4 & no $\mathrm{H}_{2} \mathrm{O}$ & $<10$ & - & - & - \\
\hline 5 & 60 & $4 \mathrm{~h}$ & 2 & no base & $5: 1$ & 0 & - & - & - \\
\hline 6 & 40 & $4 h$ & 2 & 4 & $5: 1$ & 47 & $23.4: 1$ & 26 & 97 \\
\hline 7 & 60 & $4 h$ & 2 & 4 & $5: 1$ & 77 & $30: 1$ & - & 95 \\
\hline 8 & 80 & $4 h$ & 2 & 4 & $5: 1$ & 71 & $20.9: 1$ & 8 & 94 \\
\hline
\end{tabular}

Reactions performed on $0.3 \mathrm{mmol}$ scale. dr determined by crude ${ }^{1} \mathrm{H}$ NMR. Yield shown is of isolated material after column chromatography. ee determined by SFC-MS of purified material 


\section{V. $\quad$ Absolute stereochemical assignment of trans-2 and cis-2}

Preparation of racemic mixture of trans-2 and cis-2 for SFC-MS separation conditions
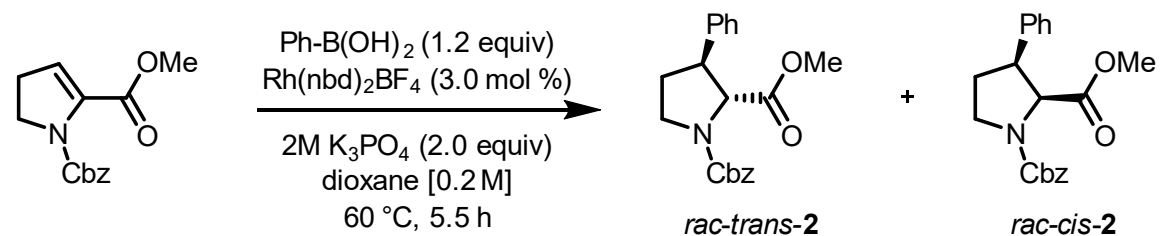

The reaction was performed according to general procedure $\mathrm{C}$ with phenylboronic acid (44 $\mathrm{mg}, 0.36$ mmol, 1.2 equiv) to afford a mixture of rac-trans-2 and rac-cis-2 as clear colorless oil (14:1 dr).

SFC-MS (Lux-4 4.6x100, $\left.3.5 \mathrm{ml} / \mathrm{min}, 15 \% \mathrm{MeOH} w / 0.1 \% \mathrm{NH}_{4} \mathrm{OH}, 2000 \mathrm{psi}\right)$

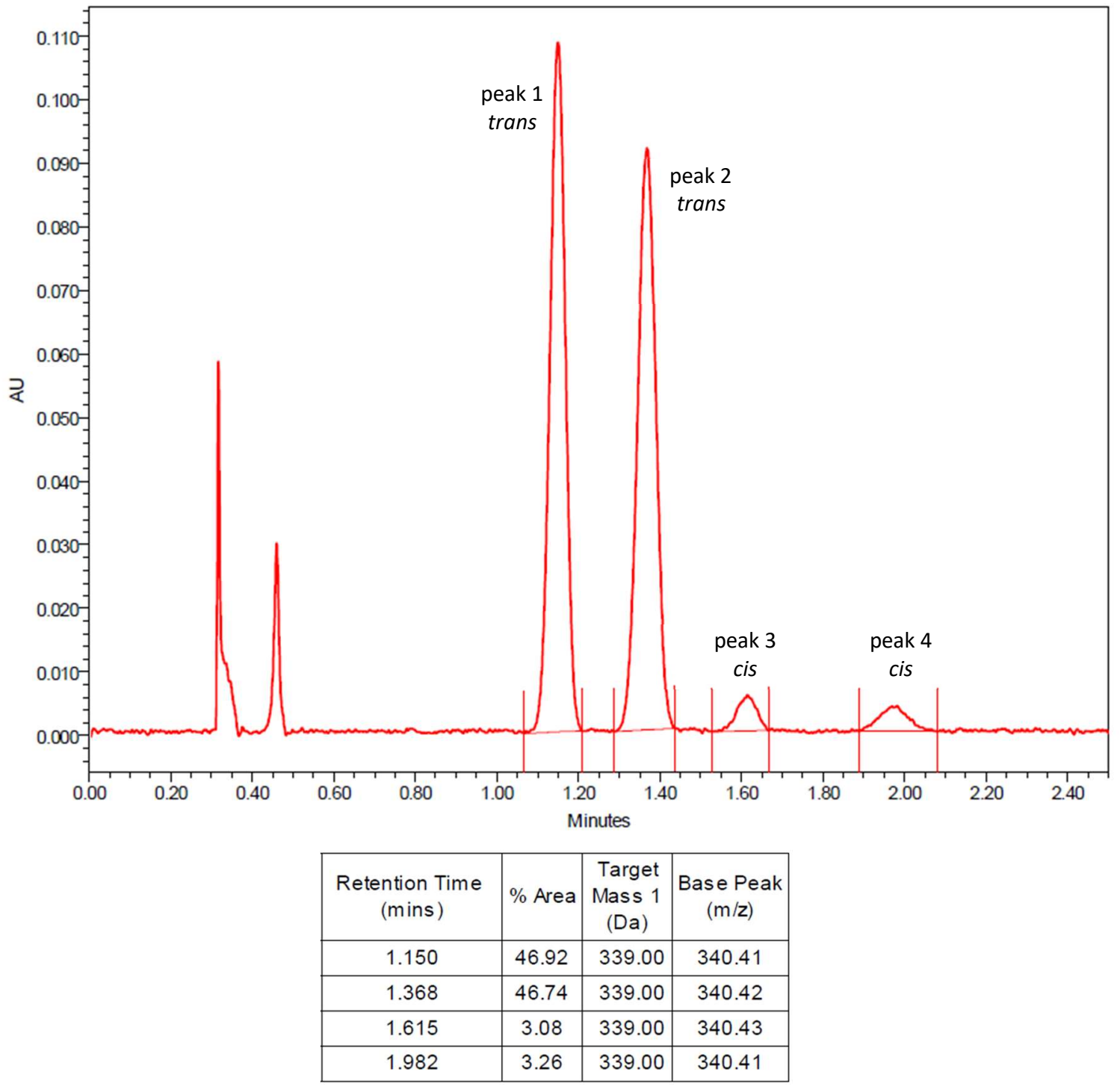


Preparation and isolation of trans-2 and cis-2

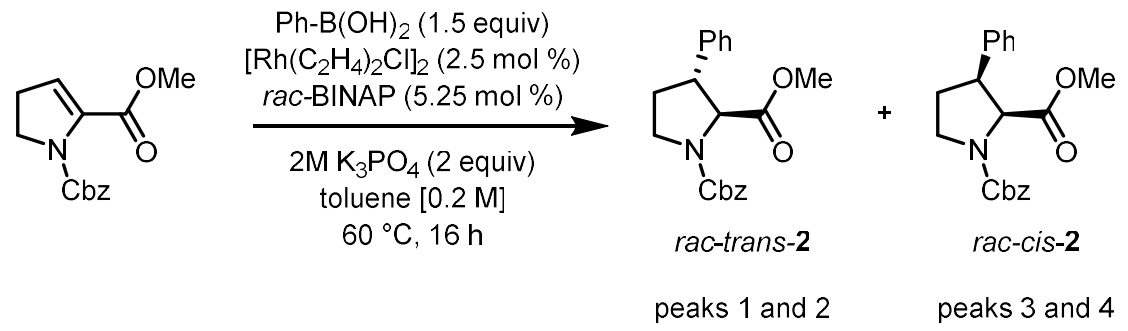

In a $\mathrm{N}_{2}$-filled glove box a $40 \mathrm{~mL}$ vial equipped with a stir bar was charged with $\left[\mathrm{Rh}\left(\mathrm{C}_{2} \mathrm{H}_{4}\right)_{2} \mathrm{Cl}\right]_{2}$ (39.0 mg, $0.100 \mathrm{mmol}, 2.50 \mathrm{~mol} \%$ ), rac-BINAP (131 mg, $0.21 \mathrm{mmol}, 5.25 \mathrm{~mol} \%$ ), and toluene (10.0 mL) and the mixture was left to stir for 10 minutes. Another $40 \mathrm{~mL}$ vial equipped with a stir bar was charged with 1 (1.05 g, $4.00 \mathrm{mmol}, 1.0$ equiv) and phenylboronic acid (732 mg, $6.00 \mathrm{mmol}, 1.5 \mathrm{equiv})$. The $\mathrm{Rh} /$ ligand solution was added to this vial and rinsed with remaining toluene (10.0 $\mathrm{mL}, 0.20 \mathrm{M}$ total). The vial was sealed with a cap containing Teflon septa and removed from the glove box. Under an inlet needle of $\mathrm{N}_{2}, \mathrm{~K}_{3} \mathrm{PO}_{4}\left(4.00 \mathrm{~mL}, 2.0 \mathrm{M}\right.$ solution, $8.00 \mathrm{mmol}, 2.0$ equiv) was added. The $\mathrm{N}_{2}$ inlet was removed and the vial was placed in an aluminum block heated to $60{ }^{\circ} \mathrm{C}$ on top of a stir plate and left to stir for $16 \mathrm{~h}$. The reaction mixture was allowed to cool to room temperature and diluted with EtOAc and a small amount of water. The layers were separated, and the aqueous layer was extracted an additional two times with EtOAc. The combined organics were dried over $\mathrm{MgSO}_{4}$, filtered, and concentrated under reduced pressure. The crude material was purified by silica gel chromatography (0-40\% ethyl acetate in hexanes) to afford a mixture of trans-2, cis-2 (1:1 dr), and $\mathbf{1}$ as clear colorless oil. This material was then purified by prep-SFC (Lux-4 21x250, $70 \mathrm{ml} / \mathrm{min}, \mathrm{MeOH}$ w/ 0.1\% $\mathrm{NH}_{4} \mathrm{OH}, 100$ bar). The four peaks were collected as viscous clear colorless oils.

Peak 1: $105 \mathrm{mg}, 7.7 \%$ yield, $>98 \%$ ee; HRESIMS m/z $340.1546 \mathrm{~m} / \mathrm{z}$ (calc'd for $\mathrm{C}_{20} \mathrm{H}_{22} \mathrm{NO}_{4}{ }^{+}$, m/z 340.1549 m/z; $\Delta$ ppm 0.9).

Peak 2: 90.5 mg, 6.7\% yield, >98\% ee; HRESIMS m/z $340.1546 \mathrm{~m} / \mathrm{z}$ (calc'd for $\mathrm{C}_{20} \mathrm{H}_{22} \mathrm{NO}_{4}{ }^{+}$, $\mathrm{m} / \mathrm{z} 340.1549 \mathrm{~m} / \mathrm{z} ; \Delta \mathrm{ppm} 0.9)$.

Peak 3: 87.8 mg, 6.5\% yield, >98\% ee; HRESIMS m/z 340.1546 m/z (calc'd for $\mathrm{C}_{20} \mathrm{H}_{22} \mathrm{NO}_{4}{ }^{+}$, $\mathrm{m} / \mathrm{z} 340.1549 \mathrm{~m} / \mathrm{z} ; \Delta \mathrm{ppm} 0.9)$.

Peak 4: $88.1 \mathrm{mg}, 6.5 \%$ yield, $>98 \%$ ee; HRESIMS m/z $340.1546 \mathrm{~m} / \mathrm{z}$ (calc'd for $\mathrm{C}_{20} \mathrm{H}_{22} \mathrm{NO}_{4}{ }^{+}$, $\mathrm{m} / \mathrm{z} 340.1549 \mathrm{~m} / \mathrm{z} ; \Delta \mathrm{ppm} 0.9)$. 
SFC traces after prep-SFC separation

SFC-MS (Lux-4 4.6x100, $\left.3.5 \mathrm{ml} / \mathrm{min}, 15 \% \mathrm{MeOH} w / 0.1 \% \mathrm{NH}_{4} \mathrm{OH}, 2000 \mathrm{psi}\right)$
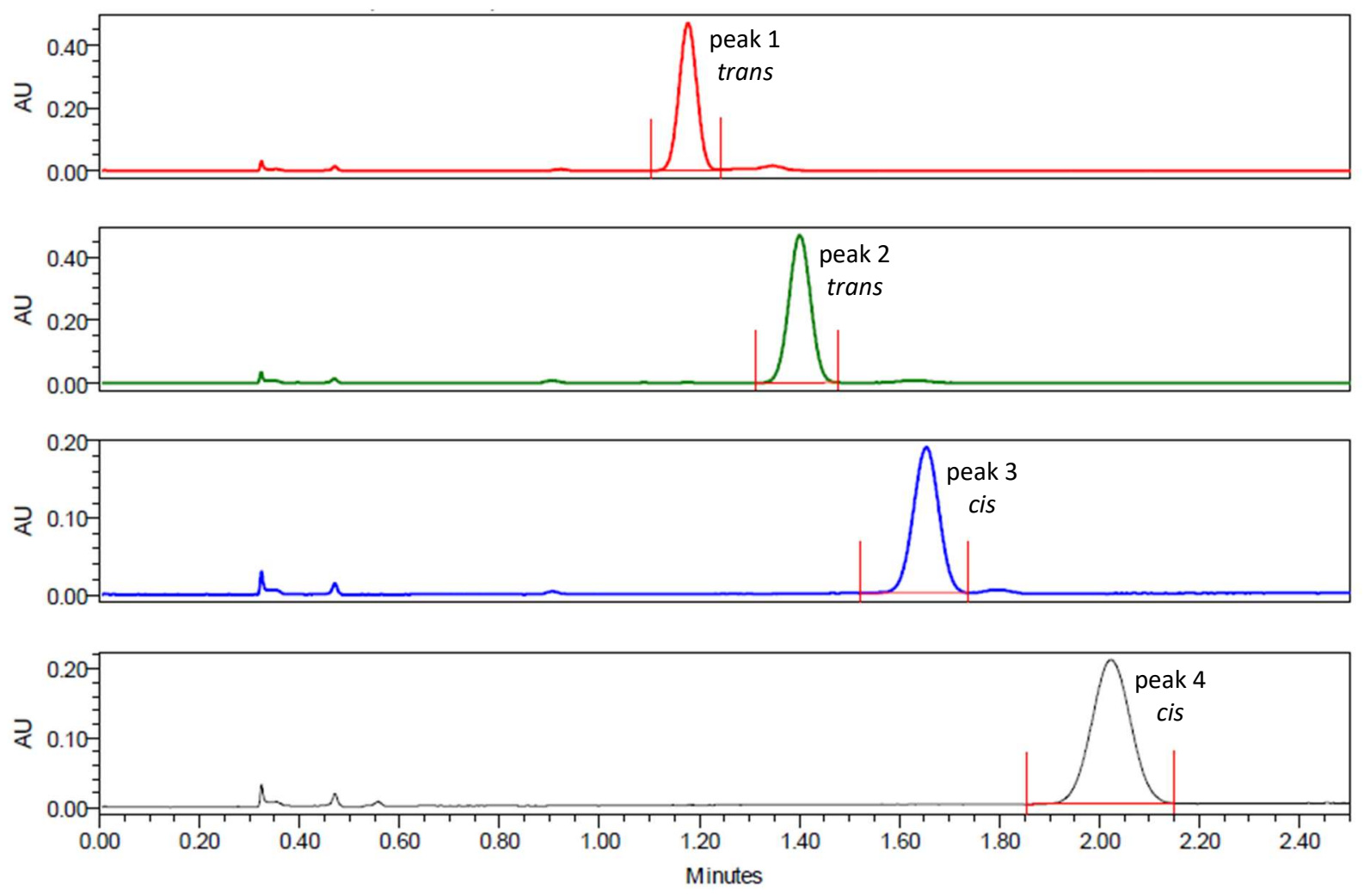

\section{ECD analysis to assign absolute stereochemistry}

ECD spectra of 2 SFC peaks 1-4 were acquired on a Jasco J-1500 CD polararimeter at $25{ }^{\circ} \mathrm{C}$ in acetonitrile at $0.13 \mathrm{c}$ from 190 to $260 \mathrm{~nm}$ scanned at $5 \mathrm{~nm} / \mathrm{min}$ with a $2 \mathrm{~nm}$ bandwidth and baseline corrected against an acetonitrile blank. Conformers of trans-2 and cis-2 utilized for DFT and TDDFT analysis were generated utilizing ForceGen 4.4 pquant conformational sampling. ${ }^{4}$ Gaussian ' 16 was utilized for all DFT calculations. ${ }^{5}$ Conformers were initially optimized in MacroModel (Schrödinger Release 2020-1: Schrödinger, LLC, New York, NY, 2020) utilizing the OPLS3e force field and all conformers below 10 $\mathrm{kcal} / \mathrm{mol}$ were submitted to geometry optimizations, frequency calculations, and TDDFT calculations to generate ECD spectra at the PCM- $\omega$ B97xD/def2-TZVP level in acetonitrile. ${ }^{6,7,8,9}$ Experimental and calculated spectra were Boltzmann averaged and scaled utilizing SpecDis and visualized in Microsoft ${ }^{\circledR}$ Excel $^{\odot} \cdot{ }^{10}$ 
Spectral data:

Peak 1 ECD (0.13c, acetonitrile) 221.8 (64.0);

Peak 2 ECD (0.13c, acetonitrile) 222.5 (-64.5);

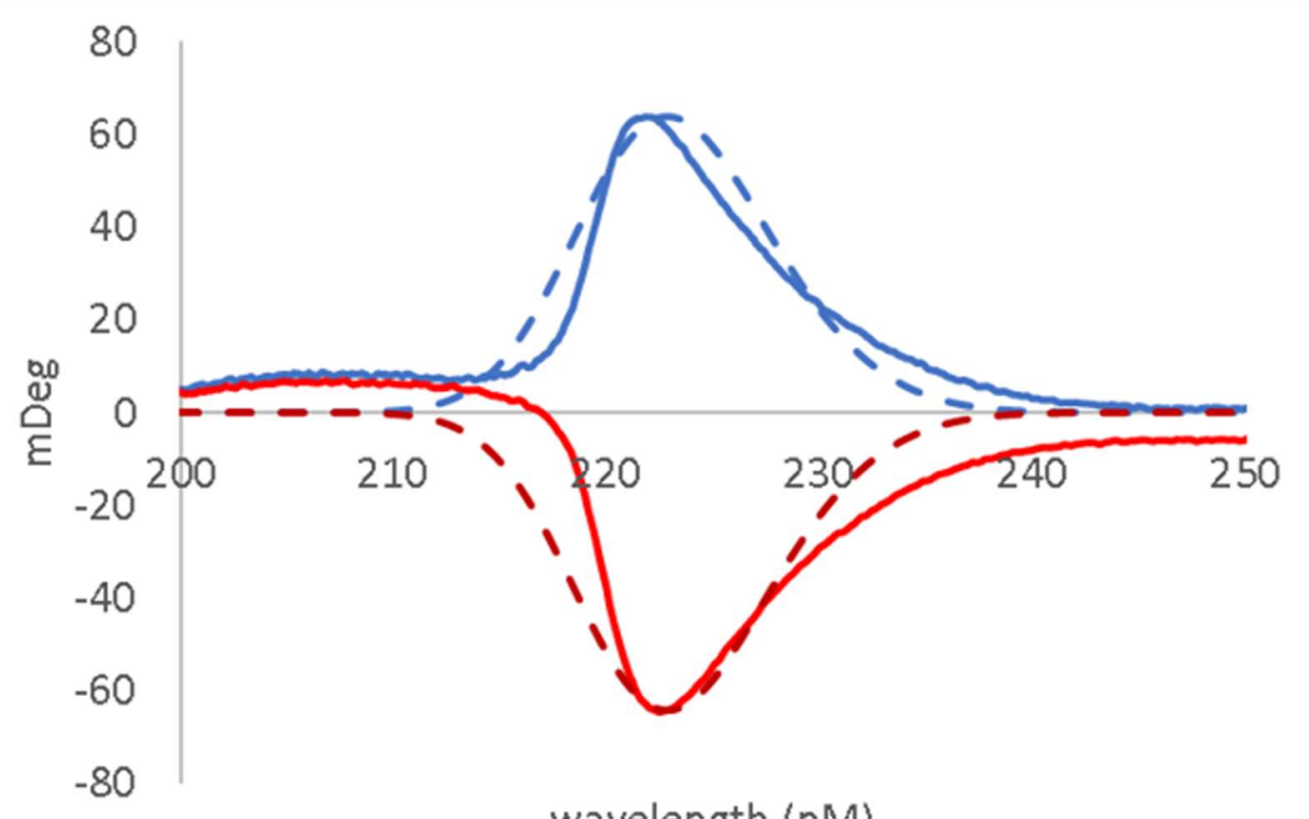

wavelength ( $\mathrm{nM}$ )

-SFC peak 1 expt. - -SFC peak 2 expt.

- - $(2 S, 3 R)$ calc'd _ - $(2 R, 3 S)$ calc'd

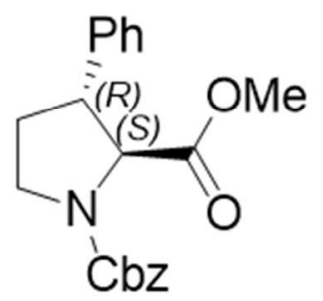

1-benzyl 2-methyl (2S,3R)-3phenylpyrrolidine-1,2dicarboxylate

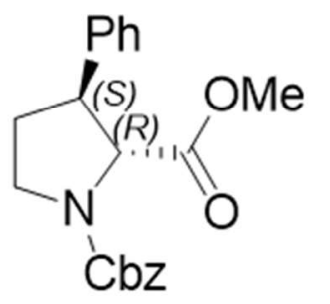

1-benzyl 2-methyl (2R,3S)-3phenylpyrrolidine-1,2dicarboxylate 
Spectral data:

Peak 3 ECD (0.13c, acetonitrile) 221.4 (48.0);

Peak 4 ECD (0.13c, acetonitrile) 222.0 (-47.4);

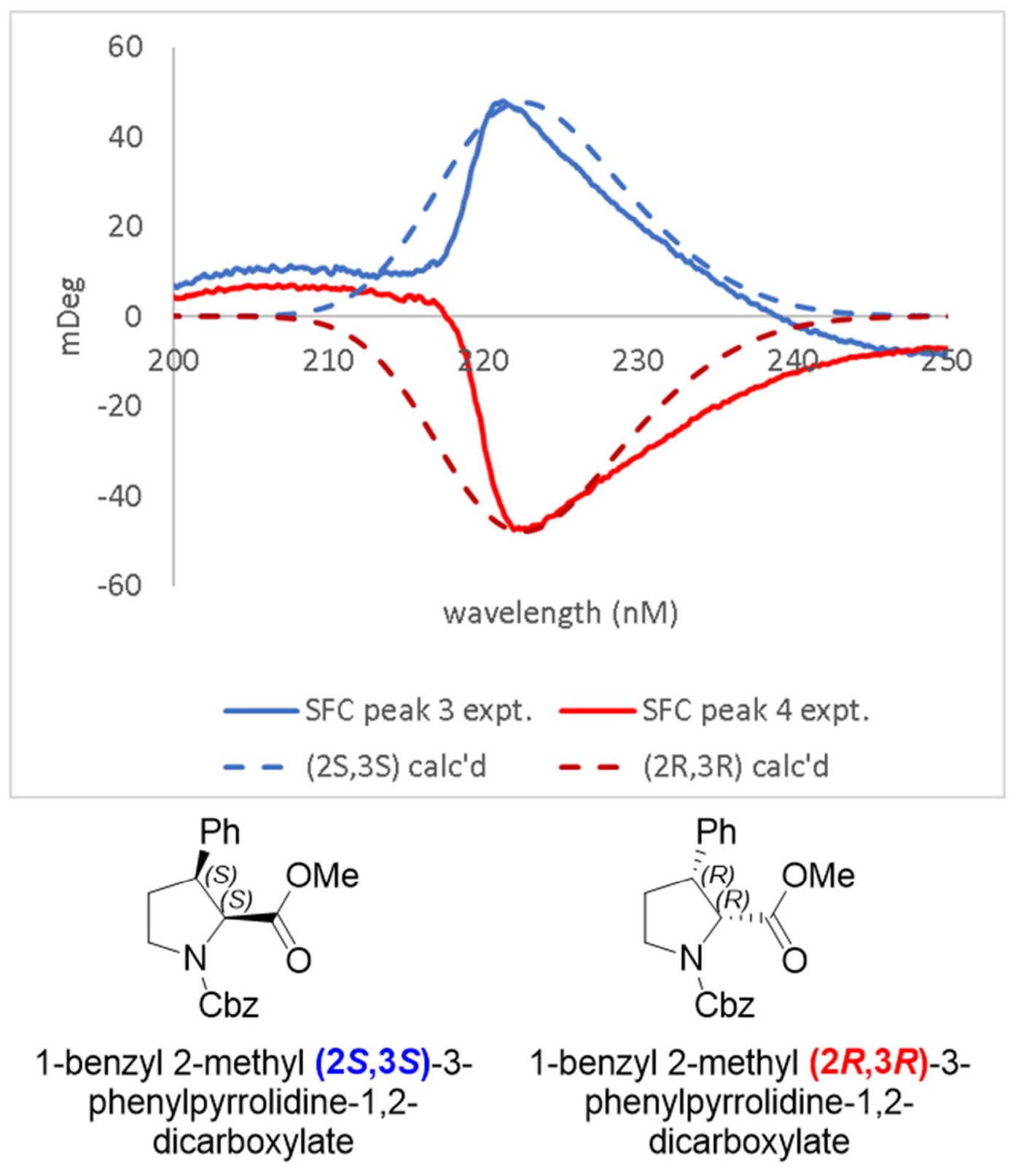




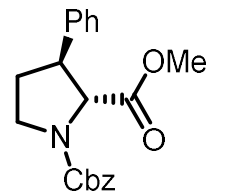

\section{1-benzyl 2-methyl (2R,3S)-3-phenylpyrrolidine-1,2-dicarboxylate (trans-2).}

${ }^{1}$ H NMR (600 MHz, DMSO-d $\left.d_{6}\right) \delta 7.48-7.38(\mathrm{~m}, 2 \mathrm{H}), 7.35(\mathrm{~d}, J=7.6 \mathrm{~Hz}, 2 \mathrm{H}), 7.33$ (s, 2H), $7.33-7.29(\mathrm{~m}, 1 \mathrm{H}), 7.30-7.29(\mathrm{~m}, 2 \mathrm{H}), 7.28-7.23(\mathrm{~m}, 1 \mathrm{H}), 5.16(\mathrm{~m}, 1.5 \mathrm{H}), 5.01(\mathrm{~d}$, $J=12.8 \mathrm{~Hz}, 0.5 \mathrm{H}), 4.31(\mathrm{~d}, J=7.4 \mathrm{~Hz}, 0.5 \mathrm{H}), 4.28(\mathrm{~d}, J=7.3 \mathrm{~Hz}, 0.5 \mathrm{H}), 3.76-3.68(\mathrm{~m}, 1 \mathrm{H}), 3.61(\mathrm{~s}$, $1.5 \mathrm{H}), 3.56-3.50(\mathrm{~m}, 1 \mathrm{H}), 3.49(\mathrm{~s}, 1.5 \mathrm{H}), 3.48-3.45(\mathrm{~m}, 0.5 \mathrm{H}), 3.45-3.41(\mathrm{~m}, 0.5 \mathrm{H}), 2.31-2.18(\mathrm{~m}$, 1H), $2.12-1.99(\mathrm{~m}, 1 \mathrm{H}) .{ }^{13} \mathrm{C}$ NMR (151 MHz, DMSO-d $) \delta 172.2,171.9,153.8,153.3,140.3,140.2$, $128.60,128.58,128.4,128.3,127.8,127.7,127.5,127.3,127.1,127.0,66.3,66.2,65.6,65.2,51.84,51.79$, $49.5,48.4,46.6,46.0,32.6,31.9$.

${ }^{1} \mathrm{H}$ NMR - 600.13 MHz - DMSO@308.0K
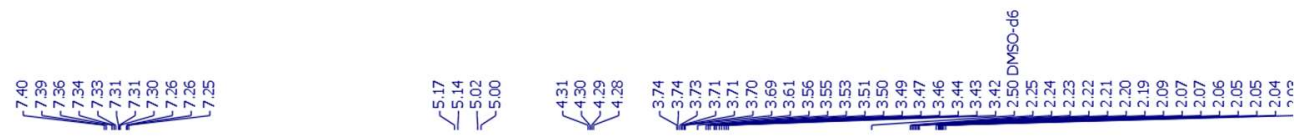<smiles>COC(=O)[C@H]1[C@@H](c2ccccc2)CCN1C(=O)[O-]</smiles>

trans-2

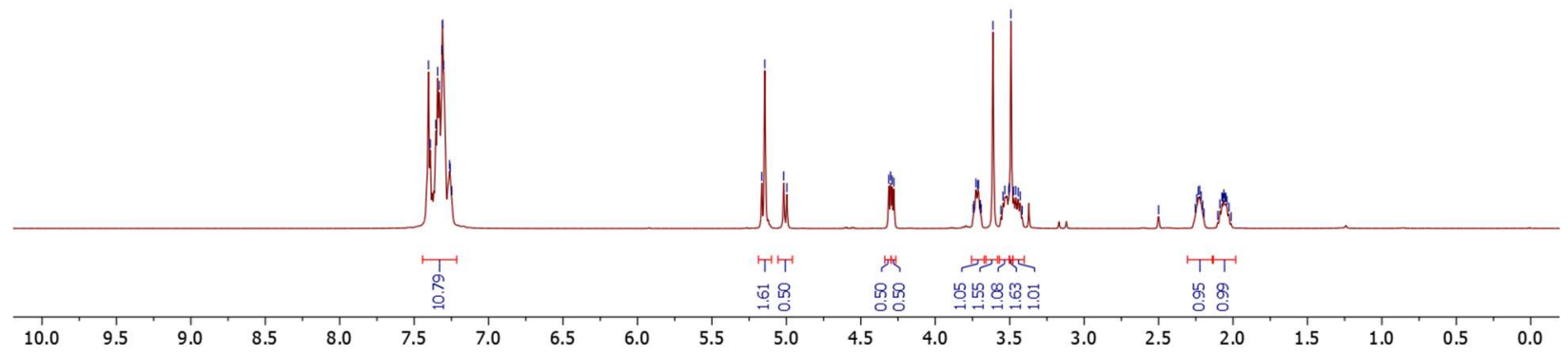


${ }^{13} \mathrm{C}$ NMR - $150.92 \mathrm{MHz}$ - DMSO@308.0K

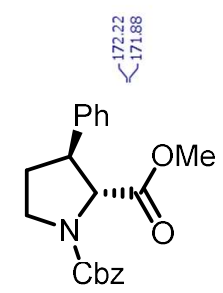

trans-2

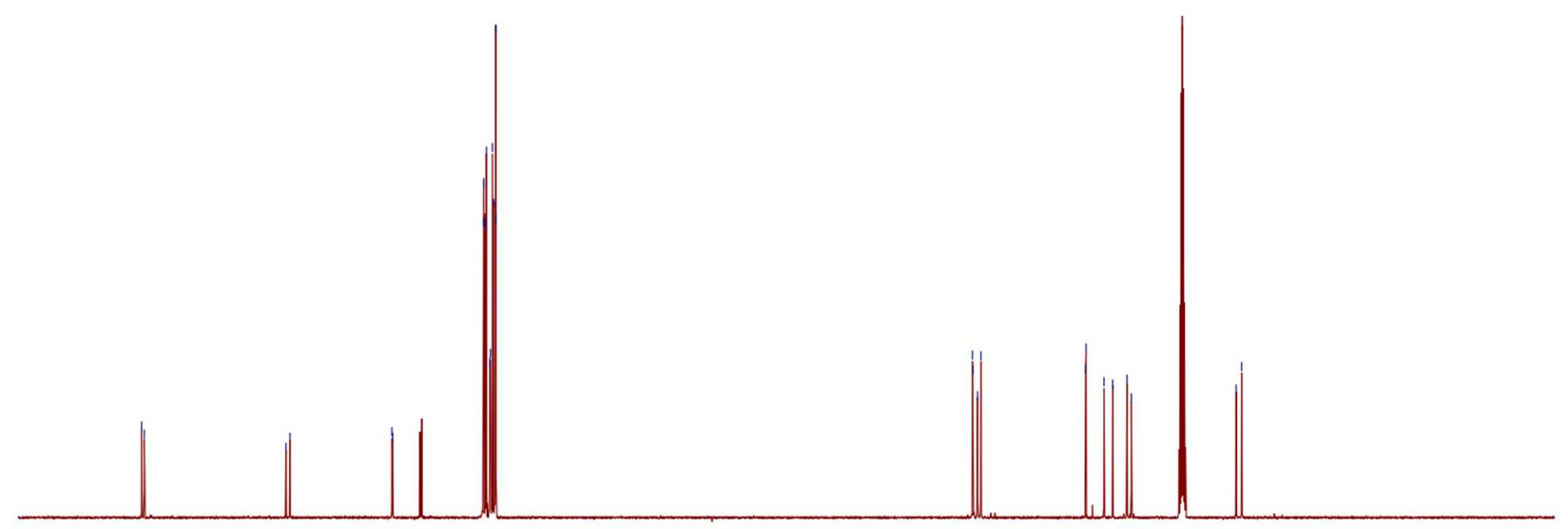

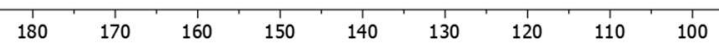
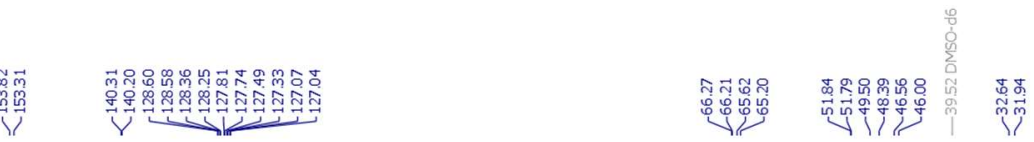
ROESY - F1: ${ }^{1} \mathrm{H}, 600.13 \mathrm{MHz}, \mathrm{F} 2:{ }^{1} \mathrm{H}, 600.13 \mathrm{MHz}-\mathrm{DMSO} @ 308.0 \mathrm{~K}$
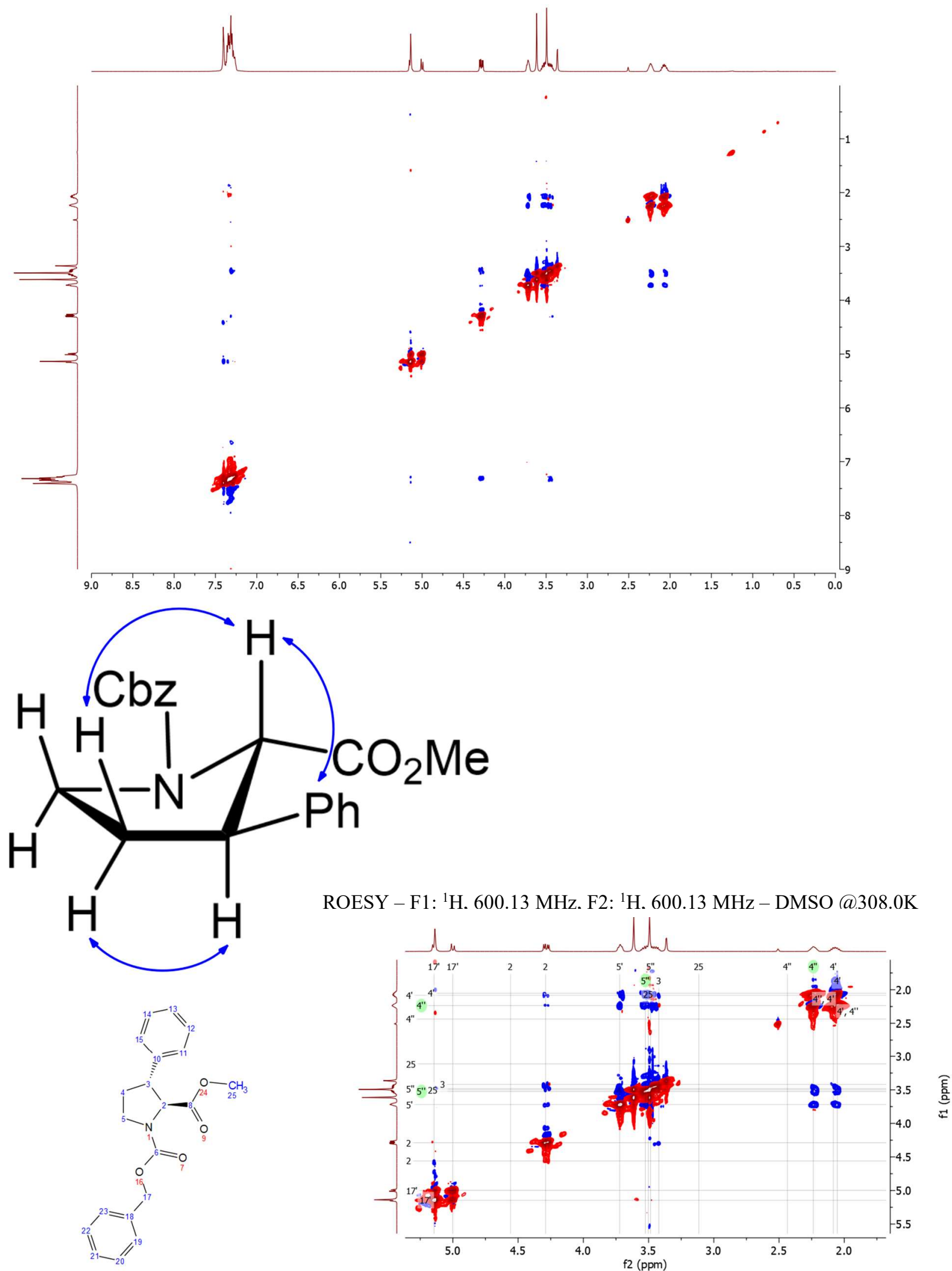


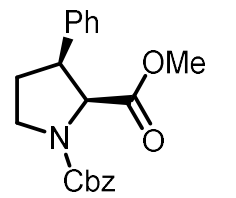

1-benzyl 2-methyl (2S,3S)-3-phenylpyrrolidine-1,2-dicarboxylate (cis-2).

${ }^{1}$ H NMR (600 MHz, DMSO- $\left.d_{6}\right) \delta 7.45$ - 7.37 (m, 2H), 7.35 - 7.29 (m, 4H), 7.29 - 7.21 (m, 4H), $5.17-5.07(\mathrm{~m}, 1.5 \mathrm{H}), 5.01(\mathrm{~d}, J=12.8 \mathrm{~Hz}, 0.5 \mathrm{H}), 4.59$ (d, $J=8.7 \mathrm{~Hz}, 0.5 \mathrm{H}), 4.54(\mathrm{~d}$, $J=8.8 \mathrm{~Hz}, 0.5 \mathrm{H}), 3.85-3.74(\mathrm{~m}, 2 \mathrm{H}), 3.47(\mathrm{~m}, 1 \mathrm{H}), 3.16(\mathrm{~s}, 1.5 \mathrm{H}), 3.11(\mathrm{~s}, 1.5 \mathrm{H}), 2.44(\mathrm{~m}, 1 \mathrm{H}), 2.08(\mathrm{dq}$, $J=12.9,6.6 \mathrm{~Hz}, 1 \mathrm{H}) .{ }^{13} \mathbf{C}$ NMR $\left(151 \mathrm{MHz}, \mathrm{DMSO}-d_{6}\right) \delta 171.10,171.96,153.9,153.3,136.8,136.7$, 136.61, 136.59, 128.4, 128.2, 128.11, 128.11, 127.82, 127.81, 127.78, 127.7, 127.5, 127.24, 127.22, 127.1, 66.14, 66.06, 63.9, 63.4, 51.0, 47.0, 46.2, 46.0, 45.7, 39.9, 39.8, 39.7, 39.5, 39.4, 39.2, 39.1, 27.8, 26.8 .

${ }^{1} \mathrm{H}$ NMR - 600.13 MHz - DMSO@308.0K

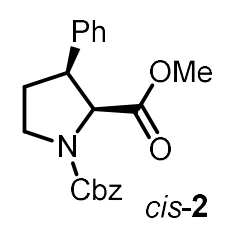

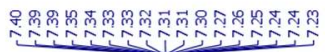
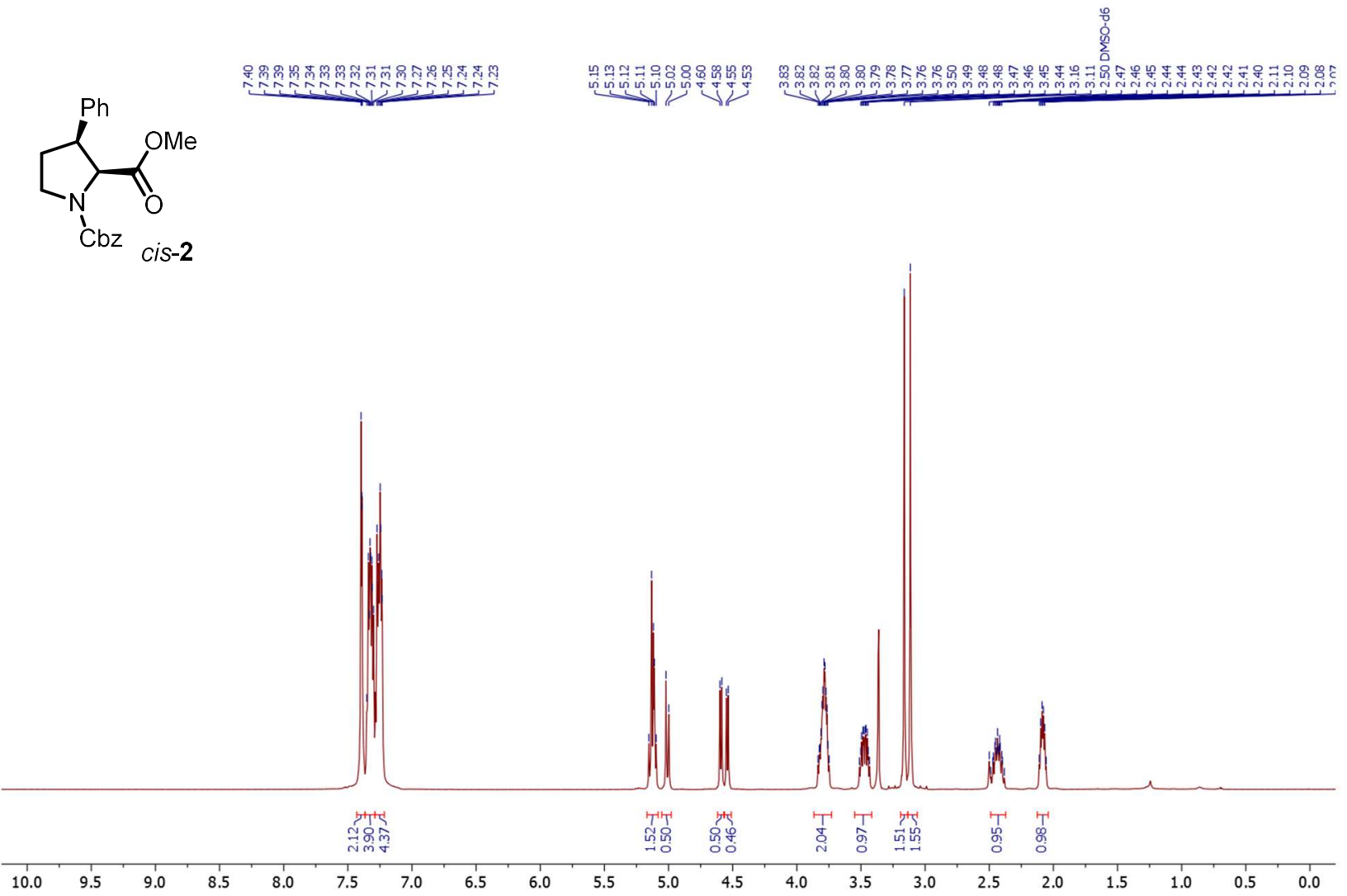
${ }^{13} \mathrm{C}$ NMR - $150.92 \mathrm{MHz}-\mathrm{DMSO} @ 308.0 \mathrm{~K}$
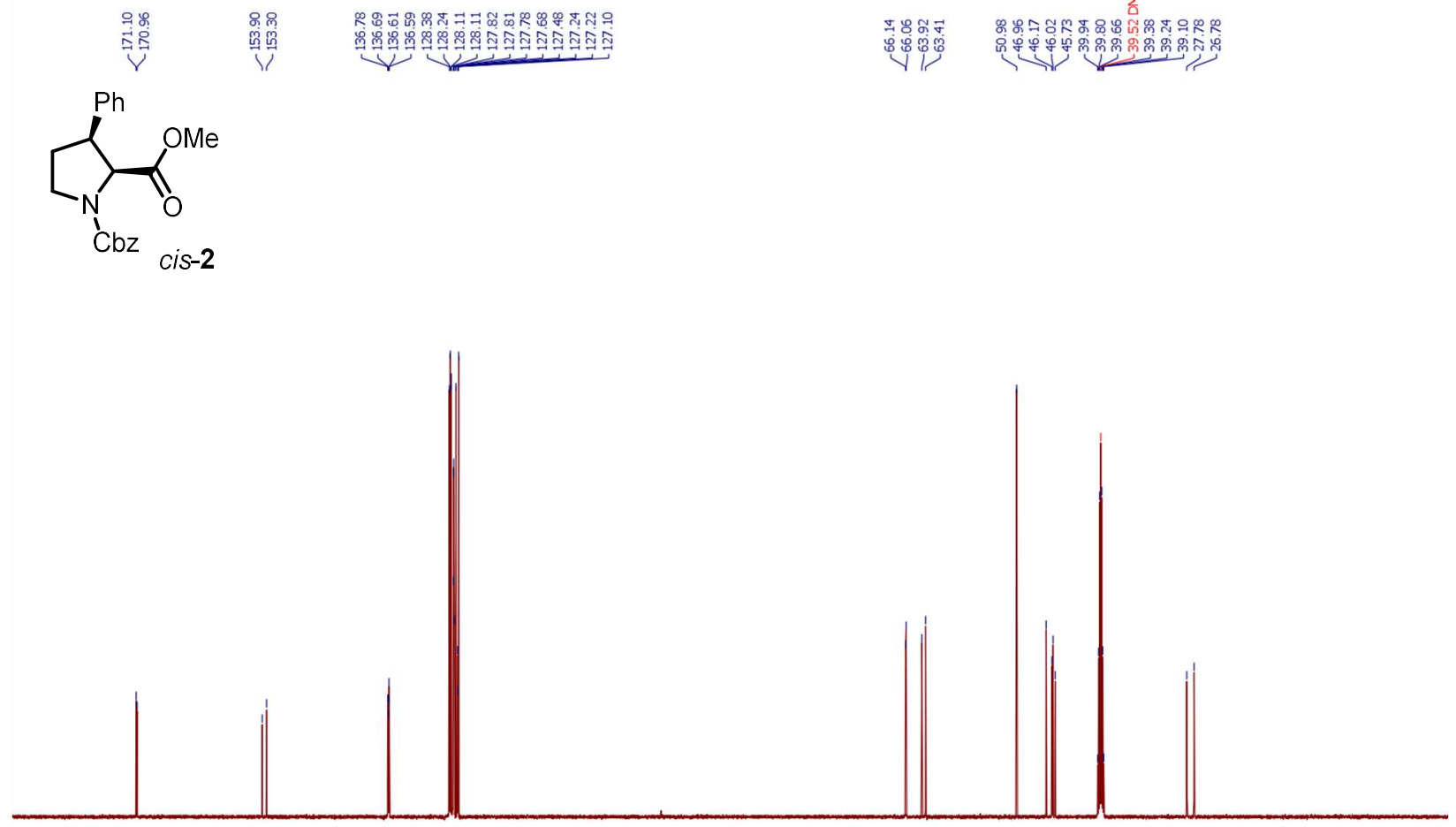

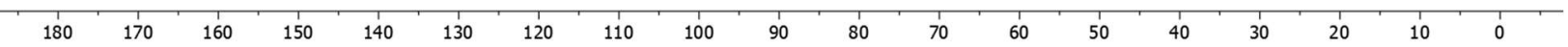


ROESY - F1: ${ }^{1} \mathrm{H}, 600.13 \mathrm{MHz}, \mathrm{F} 2:{ }^{1} \mathrm{H}, 600.13 \mathrm{MHz}-\mathrm{DMSO} @ 308.0 \mathrm{~K}$
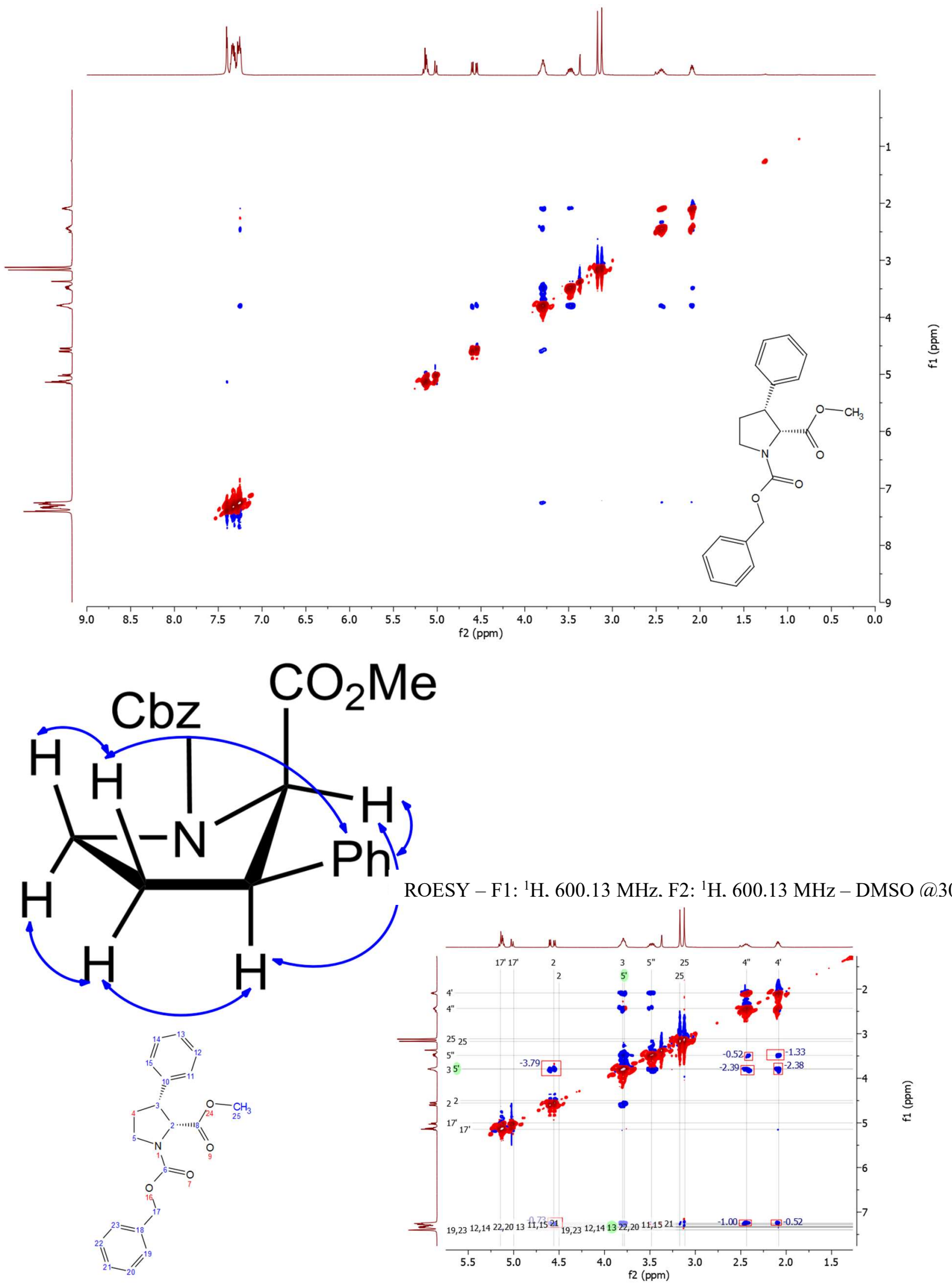

$\mathrm{CO}_{2} \mathrm{Me}$ 


\section{Characterization of reaction products and analysis of stereochemistry}

Ph 1-benzyl 2-methyl (2R,3S)-3-phenylpyrrolidine-1,2-dicarboxylate (2).

With $\mathrm{Ph}-\mathrm{B}(\mathrm{OH})_{2}$ on $4.0 \mathrm{mmol}$ scale: The reaction was performed according to general procedure A in a 40-mL vial with $(R, R)$-Ph-BPE-Rh (16 mg, $20 \mu \mathrm{mol}, 0.5 \mathrm{~mol} \%), 1$ (1.05 g, $4.0 \mathrm{mmol}, 1.0$ equiv), phenylboronic acid $\left(0.585 \mathrm{~g}, 4.80 \mathrm{mmol}, 1.2\right.$ equiv), $2 \mathrm{M} \mathrm{K}_{3} \mathrm{PO}_{4}(2 \mathrm{M}, 4.0 \mathrm{~mL}, 2.0$ equiv), and 2-MeTHF (20 mL, $0.2 \mathrm{M})$. The crude residue was purified by silica gel chromatography (0$40 \%$ EtOAc in hexanes) to afford the title compound as clear colorless oil (1.1 g, 83\% yield, 20:1 dr, 95\% ee).

With $P h-B F_{3} K$ : The reaction was performed according to general procedure A with potassium phenyltrifluoroborate $(110 \mathrm{mg}, 0.60 \mathrm{mmol}, 1.2$ equiv). The crude residue was purified by silica gel chromatography (0-40\% EtOAc in hexanes) to afford the title compound as clear colorless oil (128 $\mathrm{mg}$, $75 \%$ yield, $17: 1 \mathrm{dr}, 96 \%$ ee).

With Ph-B(pin): The reaction was performed according to general procedure A with phenylboronic acid pinacol ester (122 mg, $0.60 \mathrm{mmol}, 1.2$ equiv). The crude residue was purified by silica gel chromatography $(0-40 \%$ EtOAc in hexanes) to afford the title compound as clear colorless oil (136 mg, 80\% yield, 20:1 dr, $95 \%$ ee).

With Ph-B(neo): The reaction was performed according to general procedure A with phenylboronic acid neopentylglycol ester (114 mg, $0.60 \mathrm{mmol}, 1.2$ equiv). The crude residue was purified by silica gel chromatography $(0-40 \%$ EtOAc in hexanes) to afford the title compound as clear colorless oil (137 mg, $81 \%$ yield, $>20: 1 \mathrm{dr}, 95 \%$ ee). 
SFC-MS (Lux-4 4.6x100, $\left.3.5 \mathrm{ml} / \mathrm{min}, 15 \% \mathrm{MeOH} w / 0.1 \% \mathrm{NH}_{4} \mathrm{OH}, 2000 \mathrm{psi}\right)$
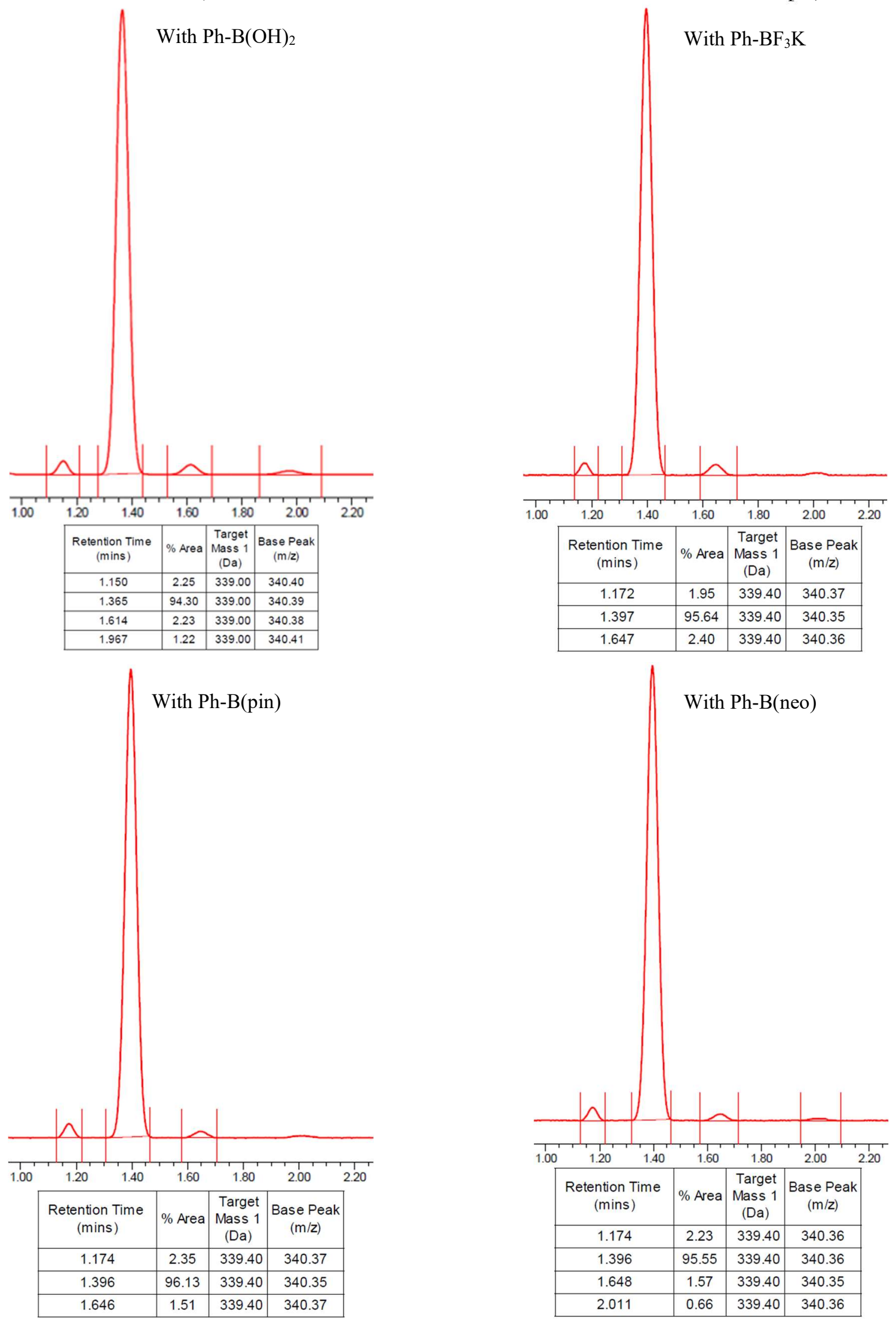
$\sum_{N_{b b z}}^{P h}$

1-benzyl 2-ethyl (2R,3S)-3-phenylpyrrolidine-1,2-dicarboxylate (3). The reaction was performed according to general procedure A on $0.30 \mathrm{mmol}$ scale with $\mathbf{S}-\mathbf{1}(83 \mathrm{mg}, 0.30$ mmol, 1.0 equiv) instead of 1 and phenylboronic acid ( $44 \mathrm{mg}, 0.36 \mathrm{mmol}, 1.2$ equiv). The crude residue was purified by silica gel chromatography $(0-25 \%$ EtOAc in hexanes) to afford the title compound as clear colorless oil (88.4 mg, 83\% yield, $13: 1 \mathrm{dr}, 97 \% e e) .{ }^{1} \mathbf{H}$ NMR $\left(600 \mathrm{MHz}\right.$, DMSO- $\left.d_{6}\right) \delta$ $7.45-7.36(\mathrm{~m}, 2 \mathrm{H}), 7.36-7.32(\mathrm{~m}, 2 \mathrm{H}), 7.32-7.28(\mathrm{~m}, 7 \mathrm{H}), 7.28-7.23(\mathrm{~m}, 1 \mathrm{H}), 5.24-5.10(\mathrm{~m}, 1 \mathrm{H})$, $5.13-5.11(\mathrm{~m}, 0.5 \mathrm{H}), 5.02(\mathrm{~d}, J=12.7 \mathrm{~Hz}, 0.5 \mathrm{H}), 4.26(\mathrm{~d}, J=7.3 \mathrm{~Hz}, 0.5 \mathrm{H}), 4.24(\mathrm{~d}, J=7.3 \mathrm{~Hz}, 0.5 \mathrm{H})$, $4.12(\mathrm{dq}, J=10.8,7.1 \mathrm{~Hz}, 0.5 \mathrm{H}), 4.05(\mathrm{dq}, J=10.9,7.1 \mathrm{~Hz}, 0.5 \mathrm{H}), 3.97(\mathrm{~m}, 1 \mathrm{H}), 3.72(\mathrm{~m}, 1 \mathrm{H}), 3.58-3.49$ (m, 1H), $3.47-3.38(\mathrm{~m}, 1 \mathrm{H}), 2.27-2.17(\mathrm{~m}, 1 \mathrm{H}), 2.14-2.02(\mathrm{~m}, 1 \mathrm{H}), 1.09(\mathrm{t}, J=7.2 \mathrm{~Hz}, 1.5 \mathrm{H}), 0.98(\mathrm{t}$, $J=7.2 \mathrm{~Hz}, 1.5 \mathrm{H}) .{ }^{13} \mathbf{C}$ NMR $\left(151 \mathrm{MHz}, \mathrm{DMSO}-d_{6}\right) \delta 171.7,171.3,153.8,153.3,140.3,140.2,136.8,136.5$, $128.53,128.51,128.3,128.2,127.8,127.7,127.5,127.3,127.12,127.09,127.04,127.03,66.3,66.1,65.7$, 65.3, 60.5, 60.4, 49.6, 48.5, 46.6, 46.0, 32.6, 31.9, 13.9, 13.8. HRESIMS $376.1521 \mathrm{~m} / \mathrm{z}$ (calc'd for $\left.\mathrm{C}_{21} \mathrm{H}_{23} \mathrm{NO}_{4} \mathrm{Na}^{+}, \mathrm{m} / \mathrm{z} 376.1525 \mathrm{~m} / \mathrm{z} ; \Delta \mathrm{ppm} 1.1\right)$

Analysis of Stereochemistry: The racemic material was prepared according to general procedure C (15:1 dr). Absolute stereochemistry was assigned by analogy (see section V).

SFC-MS: Lux-4 4.6x100, $3.5 \mathrm{ml} / \mathrm{min}, 15 \% \mathrm{MeOH} w / 0.1 \% \mathrm{NH}_{4} \mathrm{OH}, 2000 \mathrm{psi}$
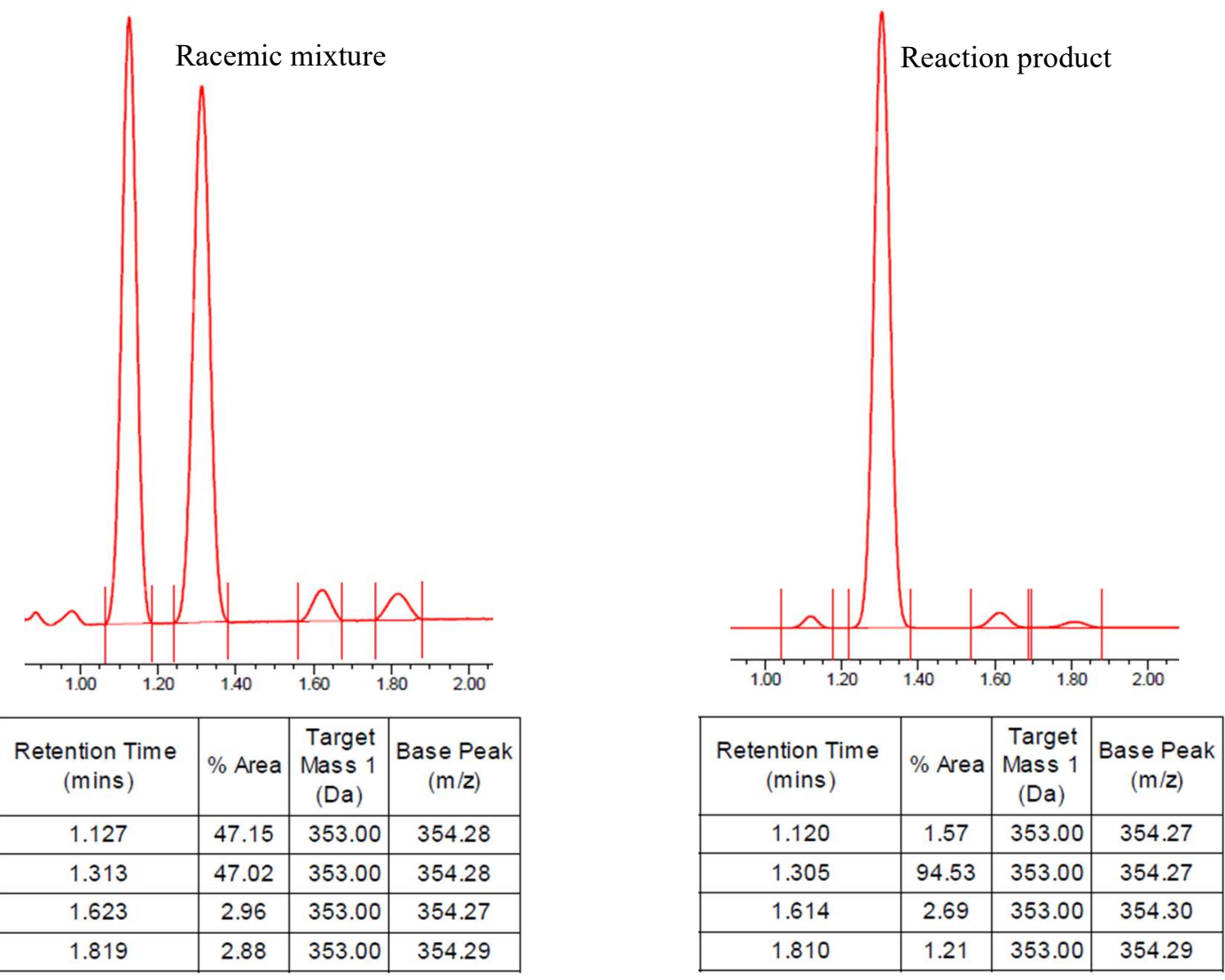


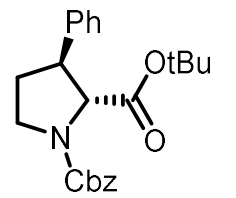

1-benzyl 2-(tert-butyl) (2R,3S)-3-phenylpyrrolidine-1,2-dicarboxylate (4). The reaction was performed according to general procedure A on $0.3 \mathrm{mmol}$ scale with $\mathbf{S - 2}(91 \mathrm{mg}, 0.30$ mmol, 1.0 equiv) instead of 1 and phenylboronic acid ( $44 \mathrm{mg}, 0.36 \mathrm{mmol}, 1.2$ equiv). The crude residue was purified by silica gel chromatography (0-25\% EtOAc in hexanes) to afford a mixture of the title compound and starting material as clear colorless oil ( $89.8 \mathrm{mg}, 84 \mathrm{wt} \% \mathbf{4}, 65 \%$ yield). This mixture was then purified by mass directed reverse phase HPLC with TFA modifier to afford the title compound as clear colorless oil (52 mg, 46\% yield, 10:1 dr, >98\% ee). Rotamer ratio is 3:1. ${ }^{1} \mathbf{H}$ NMR (600 MHz, DMSO$\left.d_{6}\right) \delta 7.43-7.36(\mathrm{~m}, 2 \mathrm{H}), 7.35-7.28(\mathrm{~m}, 4 \mathrm{H}), 7.28-7.23(\mathrm{~m}, 1 \mathrm{H}), 5.15(\mathrm{~d}, J=12.7 \mathrm{~Hz}, 0.4 \mathrm{H}), 5.13(\mathrm{~d}, J$ $=12.7 \mathrm{~Hz}, 0.4 \mathrm{H}), 5.10(\mathrm{~d}, J=12.5 \mathrm{~Hz}, 0.6 \mathrm{H}), 5.04(\mathrm{~d}, J=12.8 \mathrm{~Hz}, 0.6 \mathrm{H}), 4.14(\mathrm{~d}, J=7.4 \mathrm{~Hz}, 0.6 \mathrm{H}), 4.11$ $(\mathrm{d}, J=7.3 \mathrm{~Hz}, 0.4 \mathrm{H}), 3.79-3.62(\mathrm{~m}, 1 \mathrm{H}), 3.54-3.44(\mathrm{~m}, 1 \mathrm{H}), 3.43-3.28(\mathrm{~m}, 1 \mathrm{H}), 2.28-2.14(\mathrm{~m}, 1 \mathrm{H})$, $2.11-1.97(\mathrm{~m}, 1 \mathrm{H}), 1.32(\mathrm{~s}, 3.5 \mathrm{H}), 1.21(\mathrm{~s}, 5.5 \mathrm{H}) .{ }^{13} \mathbf{C}$ NMR (151 MHz, DMSO-d $) \delta$ 170.7, 170.3, 153.7, $153.5,140.50,140.45,136.9,136.5,128.44,128.42,128.3,128.1,127.7,127.6,127.4,127.32$, 127.25, 127.2, 127.0, 126.9, 80.6, 80.4, 66.4, 66.2, 66.0, 65.9, 49.9, 48.7, 46.6, 46.0, 32.5, 31.9, 27.5, 27.3. HRESIMS $382.2026 \mathrm{~m} / \mathrm{z}$ (calc'd for $\mathrm{C}_{23} \mathrm{H}_{28} \mathrm{NO}_{4}{ }^{+}, \mathrm{m} / \mathrm{z} 382.2018 \mathrm{~m} / \mathrm{z} ; \Delta \mathrm{ppm} 1.1$ )

Analysis of Stereochemistry: The racemic material was prepared according to general procedure C $(>20: 1$ dr). Absolute stereochemistry was assigned by analogy (see section $\mathrm{V}$ ).

SFC-MS: (R,R)-Whelk-01 4.6x100, $3.5 \mathrm{ml} / \mathrm{min}, 15 \% \mathrm{MeOH} w / 0.1 \% \mathrm{NH}_{4} \mathrm{OH}, 2000 \mathrm{psi}$

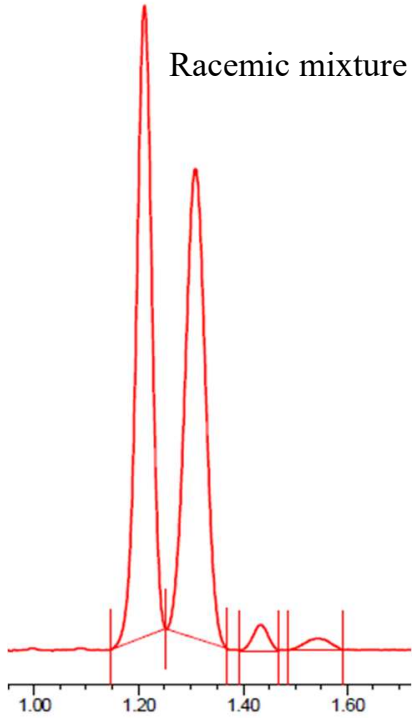

\begin{tabular}{|c|c|c|c|}
\hline $\begin{array}{c}\text { Retention Time } \\
(\mathrm{mins})\end{array}$ & \% Area & $\begin{array}{c}\text { Target } \\
\text { Mass 1 } \\
(\mathrm{Da})\end{array}$ & $\begin{array}{c}\text { Base Peak } \\
(\mathrm{m} / \mathrm{z})\end{array}$ \\
\hline 1.211 & 48.54 & 325.00 & 282.22 \\
\hline 1.309 & 47.85 & 325.00 & 282.24 \\
\hline 1.434 & 2.04 & 325.00 & 282.22 \\
\hline 1.542 & 1.57 & 325.00 & 282.23 \\
\hline
\end{tabular}

Reaction product

\begin{tabular}{|c|c|c|c|}
\hline $\begin{array}{c}\text { Retention Time } \\
\text { (mins) }\end{array}$ & \% Area & $\begin{array}{c}\text { Target } \\
\text { Mass 1 } \\
(\mathrm{Da})\end{array}$ & $\begin{array}{c}\text { Base Peak } \\
(\mathrm{m} / \mathbf{z})\end{array}$ \\
\hline 1.209 & 90.94 & 325.00 & 282.25 \\
\hline 1.433 & 1.39 & 325.00 & 282.22 \\
\hline 1.541 & 7.68 & 325.00 & 282.23 \\
\hline
\end{tabular}


$\sum_{N_{C b z}}^{P h}$

dibenzyl (2R,3S)-3-phenylpyrrolidine-1,2-dicarboxylate (5). The reaction was performed according to general procedure A on $0.3 \mathrm{mmol}$ scale with $\mathbf{S - 3}(101 \mathrm{mg}, 0.30 \mathrm{mmol}, 1.0$ equiv) instead of 1 and phenylboronic acid ( $44 \mathrm{mg}, 0.36 \mathrm{mmol}, 1.2$ equiv). The crude residue was purified by silica gel chromatography (0-25\% EtOAc in hexanes) to afford a mixture of the title compound and starting material as clear colorless oil (115.5 mg, $97 \mathrm{wt} \% \mathbf{5 , 9 0 \%}$ yield). This mixture was then purified by mass directed reverse phase HPLC with TFA modifier to afford the title compound as clear colorless oil (80 mg, 64\% yield, 17:1 dr, >98\% ee). ${ }^{1}$ H NMR (600 MHz, DMSO- $\left.d_{6}\right) \delta 7.46-7.36(\mathrm{~m}, 2 \mathrm{H})$, $7.35-7.30(\mathrm{~m}, 5 \mathrm{H}), 7.30-7.24(\mathrm{~m}, 6 \mathrm{H}), 7.23-7.19(\mathrm{~m}, 1 \mathrm{H}), 7.16-7.07$ (m, 1H), 5.17 (d, $J=12.8 \mathrm{~Hz}$, $0.5 \mathrm{H}), 5.15(\mathrm{~s}, 1 \mathrm{H}), 5.09(\mathrm{~d}, J=12.7 \mathrm{~Hz}, 0.5 \mathrm{H}), 5.06(\mathrm{~d}, J=12.6 \mathrm{~Hz}, 0.5 \mathrm{H}), 5.03(\mathrm{~s}, 1 \mathrm{H}), 4.95(\mathrm{~d}, J=12.5$ $\mathrm{Hz}, 0.5 \mathrm{H}), 4.34(\mathrm{~d}, J=5.6 \mathrm{~Hz}, 0.5 \mathrm{H}), 4.33(\mathrm{~d}, J=5.5 \mathrm{~Hz}, 0.5 \mathrm{H}), 3.73(\mathrm{dtd}, J=10.4,7.3,3.2 \mathrm{~Hz}, 1 \mathrm{H}), 3.58$ - $3.48(\mathrm{~m}, 1 \mathrm{H}), 3.48-3.39(\mathrm{~m}, 1 \mathrm{H}), 2.31-2.17(\mathrm{~m}, 1 \mathrm{H}), 2.14-1.99(\mathrm{~m}, 1 \mathrm{H}) .{ }^{13} \mathbf{C}$ NMR $(151 \mathrm{MHz}$, DMSO- $\left.d_{6}\right) \delta 171.6,171.2,153.9,153.3,140.0,139.9,136.7,136.4,135.7,135.5,128.6,128.5,128.4$, $128.3,128.0,127.9,127.8,127.72,127.69,127.6,127.5,127.3,127.23,127.21,127.1,66.3,66.2,66.0$, 65.9, 65.8, 65.3, 49.8, 48.7, 46.7, 46.1, 32.8, 32.0. HRESIMS $416.1868 \mathrm{~m} / \mathrm{z}$ (calc'd for $\mathrm{C}_{26} \mathrm{H}_{26} \mathrm{NO}_{4}{ }^{+}, \mathrm{m} / \mathrm{z}$ $416.1862 \mathrm{~m} / \mathrm{z} ; \Delta \mathrm{ppm} 2.1)$

Analysis of Stereochemistry: The racemic material was prepared according to general procedure C (13:1 dr). Absolute stereochemistry was assigned by analogy (see section $\mathrm{V}$ ).

SFC-MS: OD-3 4.6x100, $3.5 \mathrm{ml} / \mathrm{min}, 15 \% \mathrm{MeOH} w / 0.1 \% \mathrm{NH}_{4} \mathrm{OH}, 2000 \mathrm{psi}$
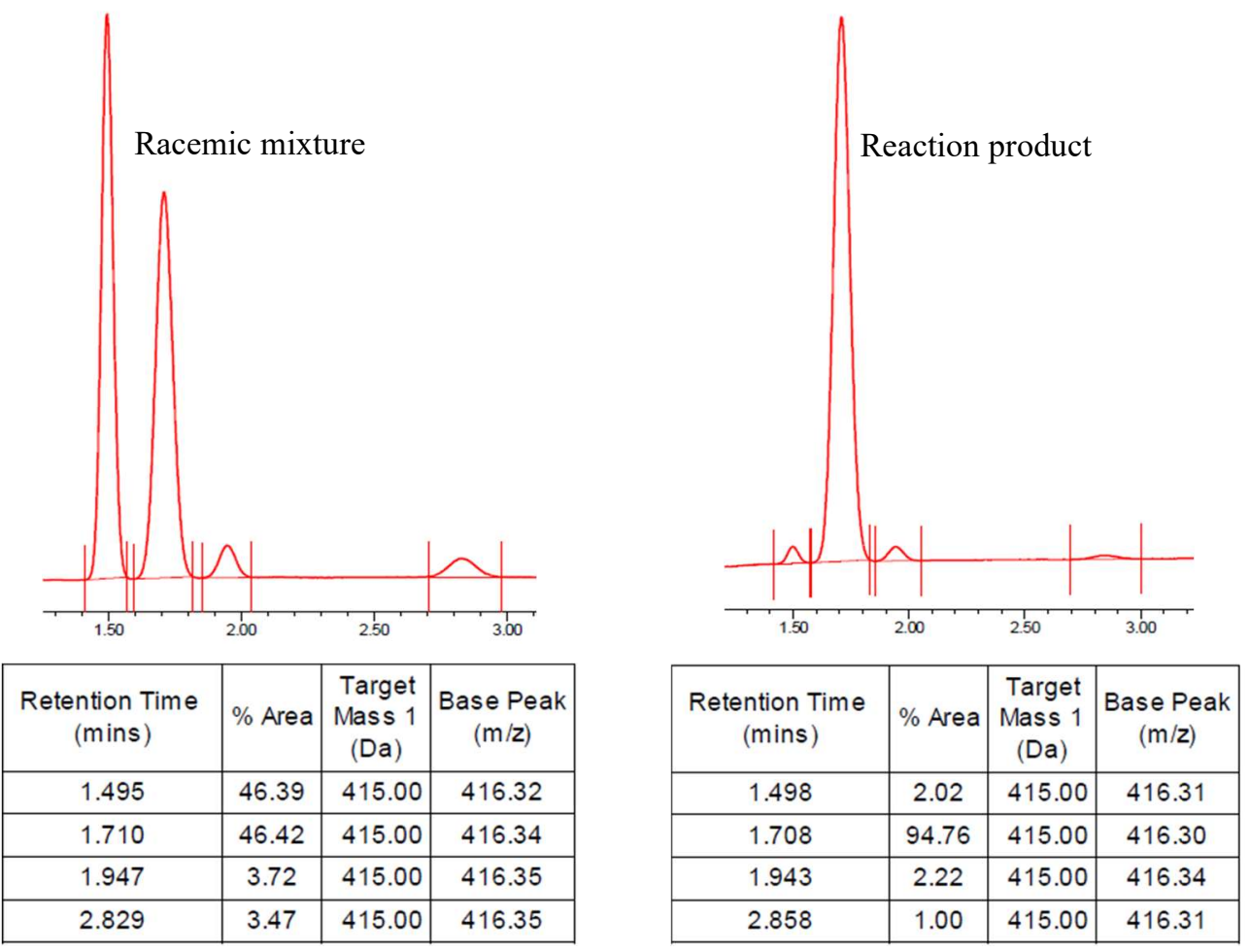


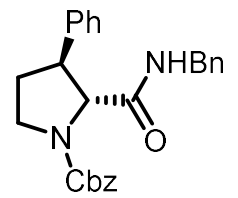

2-benzyl 1-(tert-butyl) (2R,3S)-3-phenylpyrrolidine-1,2-dicarboxylate (6). The reaction was performed according to general procedure A with $\mathbf{S - 6}(168 \mathrm{mg}, 0.50 \mathrm{mmol}, 1.0$ equiv) instead of 1 and phenylboronic acid (91 $\mathrm{mg}, 0.75 \mathrm{mmol}, 1.5$ equiv). The crude residue was purified by silica gel chromatography $(0-100 \%$ EtOAc in hexanes) to afford the title compound as white solid (194 mg, 94\% yield, 1.8:1 dr, >98\% ee). ${ }^{1}$ H NMR (600 MHz, Acetonitrile- $\left.d_{3}\right) \delta 7.41-7.38$ (m, 4H), $7.38-7.32(\mathrm{~m}, 4 \mathrm{H}), 7.59-7.23(\mathrm{~m}, 6 \mathrm{H}), 7.32-7.25(\mathrm{~m}, 4 \mathrm{H}), 7.17-7.03(\mathrm{~m}, 1 \mathrm{H}), 5.15(\mathrm{~d}, J=9.4 \mathrm{~Hz}$, 2H), $4.40(\mathrm{dd}, J=15.4,6.3 \mathrm{~Hz}, 1 \mathrm{H}), 4.32(\mathrm{~d}, J=6.3 \mathrm{~Hz}, 1 \mathrm{H}), 4.27-4.22(\mathrm{~m}, 1 \mathrm{H}), 3.77$ (ddd, $J=12.3,7.8$, $4.8 \mathrm{~Hz}, 1 \mathrm{H}), 3.60(\mathrm{dt}, J=10.9,7.5 \mathrm{~Hz}, 1 \mathrm{H}), 3.56(\mathrm{~d}, J=7.1 \mathrm{~Hz}, 1 \mathrm{H}), 3.56-3.53(\mathrm{~m}, 1 \mathrm{H}), 2.29(\mathrm{dq}, J=$ 12.4, $6.1 \mathrm{~Hz}, 1 \mathrm{H}), 2.03(\mathrm{dq}, J=12.6,7.9 \mathrm{~Hz}, 1 \mathrm{H}) .{ }^{13} \mathbf{C}$ NMR $\left(600 \mathrm{MHz}, \mathrm{DMSO}-d_{6}\right) \delta 170.9,153.7,141.3$, 139.1, 136.7, 128.2, 128.0, 127.8, 127.6, 127.3, 127.1, 126.9, 126.8, 126.4, 126.3, 66.4, 66.1, 65.9, 49.5, 48.3, 46.4, 45.9, 42.0, 32.0, 31.2. HRESIMS $415.2018 \mathrm{~m} / \mathrm{z}$ (calc'd for $\mathrm{C}_{26} \mathrm{H}_{27} \mathrm{~N}_{2} \mathrm{O}_{3}{ }^{+}, \mathrm{m} / \mathrm{z} 415.2022 \mathrm{~m} / \mathrm{z}$; $\Delta$ ppm 1.0)

Analysis of Stereochemistry: The racemic material was prepared according to general procedure C $(>20: 1$ dr). Absolute stereochemistry was assigned by analogy (see section V).

SFC-MS AS-3 4.6x100, $3.5 \mathrm{ml} / \mathrm{min}, 10 \% \mathrm{MeOH} w / 0.1 \% \mathrm{NH}_{4} \mathrm{OH}, 2000 \mathrm{psi}$
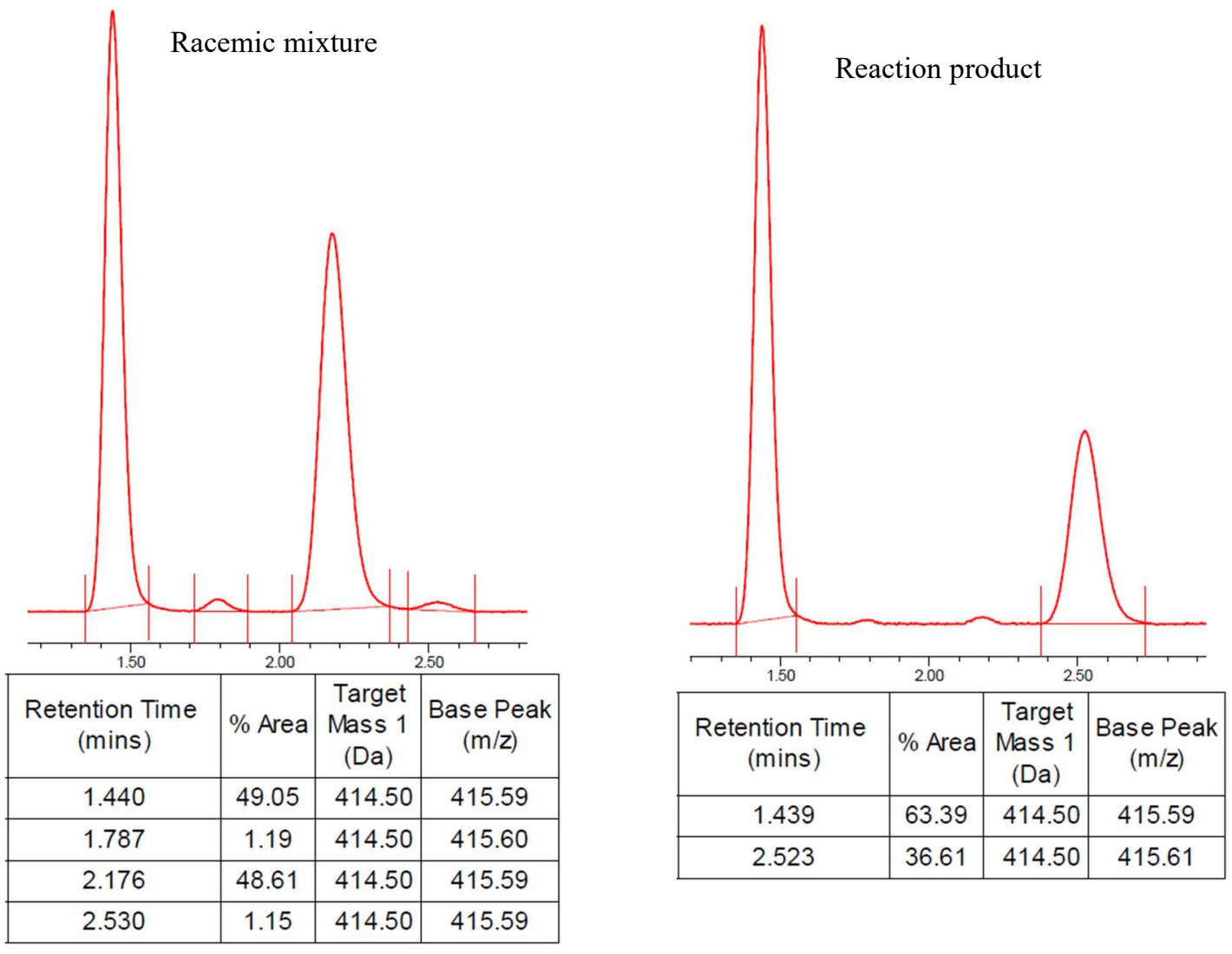
$\sum_{\text {Boc }}^{P h} \sum_{O}^{O B n}$

2-benzyl 1-(tert-butyl) (2R,3S)-3-phenylpyrrolidine-1,2-dicarboxylate (7). The reaction was performed according to general procedure A with $\mathbf{S}-\mathbf{4}$ (152 $\mathrm{mg}, 0.50 \mathrm{mmol}, 1.0$ equiv) instead of 1 and phenylboronic acid ( $73 \mathrm{mg}, 0.60 \mathrm{mmol}, 1.2$ equiv). The crude residue was purified by silica gel chromatography (0-25\% EtOAc in hexanes) to afford the title compound as clear colorless oil (164.5 mg, $84 \mathrm{wt} \%$ 7, 73\% yield, 14:1 dr, 96\% ee). This mixture was then purified by mass directed reverse phase HPLC with TFA modifier to afford the title compound as clear colorless oil (118 mg, $67 \%$ yield, $14: 1 \mathrm{dr}>98 \%$ ee). Rotamer ratio is $2: 1 .{ }^{1} \mathbf{H}$ NMR (600 MHz, DMSO- $\left.d_{6}\right) \delta 7.34-7.28(\mathrm{~m}$, $5 \mathrm{H}), 7.29-7.23(\mathrm{~m}, 3 \mathrm{H}), 7.23-7.17(\mathrm{~m}, 2 \mathrm{H}), 5.19(\mathrm{~d}, J=13.5 \mathrm{~Hz}, 0.33 \mathrm{H}), 5.17(\mathrm{~d}, J=12.9 \mathrm{~Hz}, 0.66 \mathrm{H})$, $5.07(\mathrm{~d}, J=12.5 \mathrm{~Hz}, 0.66 \mathrm{H}), 5.04(\mathrm{~d}, J=12.6 \mathrm{~Hz}, 0.33 \mathrm{H}), 4.23(\mathrm{~d}, J=7.5 \mathrm{~Hz}, 0.33 \mathrm{H}), 4.18(\mathrm{~d}, J=7.8 \mathrm{~Hz}$, $0.66 \mathrm{H}), 3.67-3.58(\mathrm{~m}, 1 \mathrm{H}), 3.49-3.33(\mathrm{~m}, 2 \mathrm{H}), 2.28-2.14(\mathrm{~m}, 1 \mathrm{H}), 2.10-1.93(\mathrm{~m}, 1 \mathrm{H}), 1.43(\mathrm{~s}, 3 \mathrm{H})$, 1.31 (s, 6H). ${ }^{13} \mathbf{C}$ NMR (151 MHz, DMSO-d $d_{6} \delta 171.9,171.4,153.3,152.6,140.2,140.0,135.8,135.6$, $128.5,128.3,128.2,128.14,128.07,128.0,127.94,127.87,127.8,127.5,127.3,127.2,127.04,126.99$, 79.17, 79.15, 65.9, 65.7, 65.5, 49.8, 48.6, 46.2, 46.1, 32.7, 32.0, 28.0, 27.8. HRESIMS $382.2018 \mathrm{~m} / \mathrm{z}$ (calc'd for $\mathrm{C}_{23} \mathrm{H}_{28} \mathrm{NO}_{4}{ }^{+}, \mathrm{m} / \mathrm{z} 382.2018 \mathrm{~m} / \mathrm{z} ; \Delta \mathrm{ppm} 3.4$ )

Analysis of Stereochemistry: The racemic material was prepared according to general procedure C (17.5:1 dr). Absolute stereochemistry was assigned by analogy (see section $\mathrm{V}$ ).

SFC-MS Lux-2 4.6x100, $3.5 \mathrm{ml} / \mathrm{min}, 20 \% \mathrm{MeOH} w / 0.1 \% \mathrm{NH}_{4} \mathrm{OH}, 2000 \mathrm{psi}$
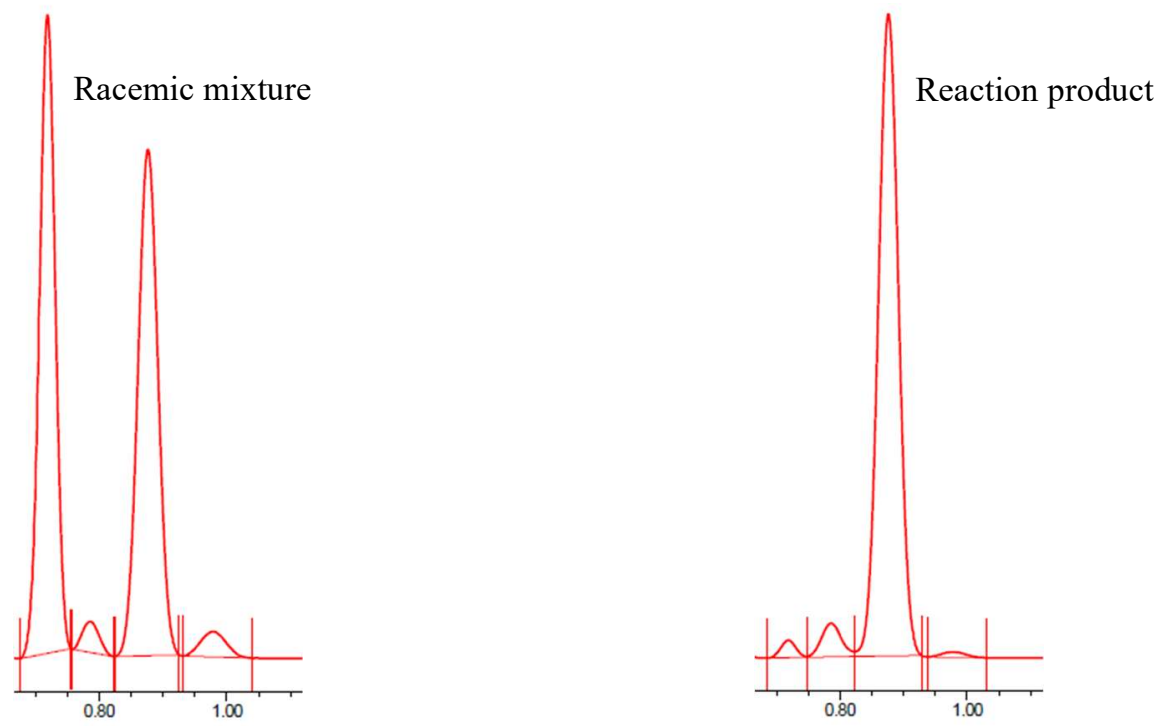

\begin{tabular}{|c|c|c|c|}
\hline $\begin{array}{c}\text { Retention Time } \\
\text { (mins) }\end{array}$ & \% Area & $\begin{array}{c}\text { Target } \\
\text { Mass 1 } \\
(\mathrm{Da})\end{array}$ & $\begin{array}{c}\text { Base Peak } \\
(\mathrm{m} / \mathbf{z})\end{array}$ \\
\hline 0.719 & 47.04 & 281.00 & 282.27 \\
\hline 0.787 & 2.41 & 281.00 & 282.22 \\
\hline 0.876 & 47.58 & 281.00 & 282.27 \\
\hline 0.978 & 2.97 & 281.00 & 282.25 \\
\hline
\end{tabular}

\begin{tabular}{|c|c|c|c|}
\hline $\begin{array}{c}\text { Retention Time } \\
\text { (mins) }\end{array}$ & \% Area & $\begin{array}{c}\text { Target } \\
\text { Mass 1 } \\
(\mathrm{Da})\end{array}$ & $\begin{array}{c}\text { Base Peak } \\
(\mathrm{m} / \mathrm{z})\end{array}$ \\
\hline 0.719 & 1.88 & 281.00 & 282.24 \\
\hline 0.786 & 4.77 & 281.00 & 282.24 \\
\hline 0.876 & 92.45 & 281.00 & 282.27 \\
\hline 0.978 & 0.90 & 281.00 & 236.35 \\
\hline
\end{tabular}




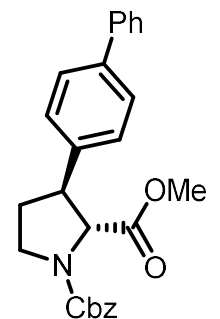

1-benzyl 2-methyl (2R,3S)-3-([1,1'-biphenyl]-4-yl)pyrrolidine-1,2-dicarboxylate (8). The reaction was performed according to general procedure A with 4-biphenylboronic acid (149 $\mathrm{mg}, 0.75 \mathrm{mmol}, 1.5$ equiv). The crude residue was purified by silica gel chromatography ( 0 $40 \%$ EtOAc in hexanes) to afford the title compound as viscous clear colorless oil (168.8 mg, 81\% yield, 19:1 dr, 95\% ee). ${ }^{1} \mathbf{H}$ NMR (600 MHz, DMSO- $\left.d_{6}\right) \delta 7.66(\mathrm{~d}, J=3.1 \mathrm{~Hz}$, 1H), $7.64(\mathrm{~d}, J=2.5 \mathrm{~Hz}, 1 \mathrm{H}), 7.63(\mathrm{~d}, J=4.4 \mathrm{~Hz}, 1 \mathrm{H}), 7.61(\mathrm{~d}, J=4.4 \mathrm{~Hz}, 1 \mathrm{H}), 7.48-7.43(\mathrm{~m}, 2 \mathrm{H}), 7.42$ $-7.37(\mathrm{~m}, 4 \mathrm{H}), 7.37-7.33(\mathrm{~m}, 2 \mathrm{H}), 7.32-7.27(\mathrm{~m}, 2 \mathrm{H}), 5.19-5.13(\mathrm{~m}, 1.5 \mathrm{H}), 5.02(\mathrm{~d}, J=12.7 \mathrm{~Hz}, 0.5 \mathrm{H})$, $4.34(\mathrm{~d}, J=7.3 \mathrm{~Hz}, 0.5 \mathrm{H}), 4.32(\mathrm{~d}, J=7.2 \mathrm{~Hz}, 0.5 \mathrm{H}), 3.78-3.68(\mathrm{~m}, 1 \mathrm{H}), 3.63(\mathrm{~s}, 1.5 \mathrm{H}), 3.60-3.52(\mathrm{~m}$, 1H), $3.51(\mathrm{~s}, 1.5 \mathrm{H}), 3.50-3.44(\mathrm{~m}, 1 \mathrm{H}), 2.31-2.18(\mathrm{~m}, 1 \mathrm{H}), 2.14-2.03(\mathrm{~m}, 1 \mathrm{H}) .{ }^{13} \mathrm{C}$ NMR $(151 \mathrm{MHz}$, DMSO-d $\left.{ }_{6}\right) \delta 172.3,171.9,153.9,153.4,139.7,139.5,139.4,139.0,136.8,136.5,128.9,128.4,128.3$, $127.9,127.8,127.74,127.70,127.5,127.4,126.92$, 126.90, 126.5, 66.32, 66.25, 65.6, 65.2, 51.92, 51.86, 49.2, 48.1, 46.6, 46.1, 32.7, 32.0. HRESIMS $416.1869 \mathrm{~m} / \mathrm{z}$ (calc'd for $\mathrm{C}_{26} \mathrm{H}_{26} \mathrm{NO}_{4}{ }^{+}, \mathrm{m} / \mathrm{z} 416.1862 \mathrm{~m} / \mathrm{z}$; $\Delta$ ppm 1.7)

Analysis of Stereochemistry: The racemic material was prepared according to general procedure C (17:1 dr). Absolute stereochemistry was assigned by analogy (see section V).

SFC-MS: Lux-4 4.6x100, $3.5 \mathrm{ml} / \mathrm{min}, 20 \% \mathrm{MeOH} w / 0.1 \% \mathrm{NH}_{4} \mathrm{OH}, 2000 \mathrm{psi}$
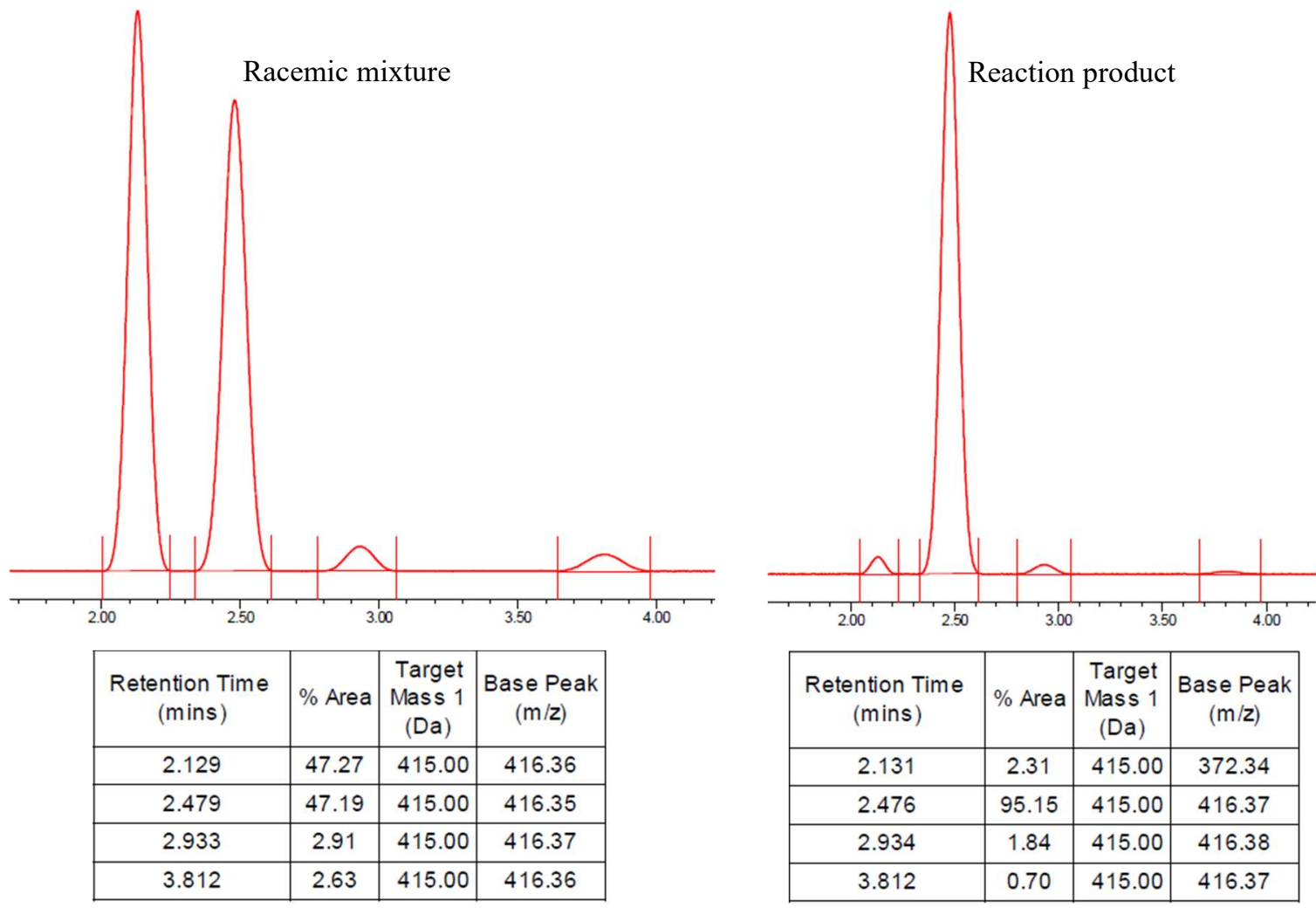


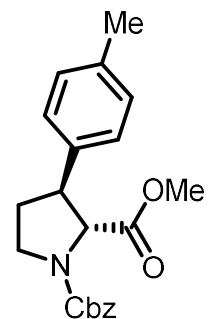

1-benzyl 2-methyl (2R,3S)-3-(p-tolyl)pyrrolidine-1,2-dicarboxylate (9). The reaction was performed according to general procedure A with $p$-tolylboronic acid $(82 \mathrm{mg}, 0.60 \mathrm{mmol}$, 1.2 equiv). The crude residue was purified by silica gel chromatography (0-40\% EtOAc in hexanes) to afford the title compound as clear colorless oil (131.2 mg, 74\% yield, 20:1 dr, 95\% ee). ${ }^{1} \mathbf{H}$ NMR (600 MHz, DMSO- $\left.d_{6}\right) \delta 7.47$ - $7.38(\mathrm{~m}, 2 \mathrm{H}), 7.37-7.33(\mathrm{~m}, 1 \mathrm{H}), 7.32$ $-7.26(\mathrm{~m}, 2 \mathrm{H}), 7.21-7.16(\mathrm{~m}, 2 \mathrm{H}), 7.16-7.11(\mathrm{~m}, 2 \mathrm{H}), 5.20-5.08(\mathrm{~m}, 1.5 \mathrm{H}), 5.00(\mathrm{~d}, J=12.7 \mathrm{~Hz}, 0.5 \mathrm{H})$, $4.26(\mathrm{~d}, J=7.3 \mathrm{~Hz}, 0.5 \mathrm{H}), 4.24(\mathrm{~d}, J=7.2 \mathrm{~Hz}, 0.5 \mathrm{H}), 3.70(\mathrm{dtd}, J=11.2,7.8,3.6 \mathrm{~Hz}, 1 \mathrm{H}), 3.61(\mathrm{~s}, 1.5 \mathrm{H})$, $3.55-3.50(\mathrm{~m}, 1 \mathrm{H}), 3.49(\mathrm{~s}, 1.5 \mathrm{H}), 3.45-3.35(\mathrm{~m}, 1 \mathrm{H}), 2.27(\mathrm{~s}, 3 \mathrm{H}), 2.23-2.13(\mathrm{~m}, 1 \mathrm{H}), 2.10-1.97(\mathrm{~m}$, 1H). ${ }^{13} \mathbf{C}$ NMR $\left(151 \mathrm{MHz}, \mathrm{DMSO}-d_{6}\right) \delta 172.3,171.9,153.8,153.3,137.2,137.1,136.8,136.5,136.2$, 129.2, 129.1, 128.4, 128.2, 127.8, 127.7, 127.5, 127.3, 126.93, 126.90, 66.3, 66.2, 65.7, 65.3, 51.8, 51.8, 49.2, 48.1, 46.6, 46.0, 32.6, 32.0, 20.5. HRESIMS $354.1714 \mathrm{~m} / \mathrm{z}$ (calc'd for $\mathrm{C}_{21} \mathrm{H}_{24} \mathrm{NO}_{4}{ }^{+}, \mathrm{m} / \mathrm{z} 354.1705$ $\mathrm{m} / \mathrm{z} ; \Delta \mathrm{ppm} 2.5)$

Analysis of Stereochemistry: The racemic material was prepared according to general procedure C (14:1 dr). Absolute stereochemistry was assigned by analogy (see section V).

SFC-MS: Lux-4 4.6x100, $3.5 \mathrm{ml} / \mathrm{min}, 15 \% \mathrm{MeOH} w / 0.1 \% \mathrm{NH}_{4} \mathrm{OH}, 2000 \mathrm{psi}$
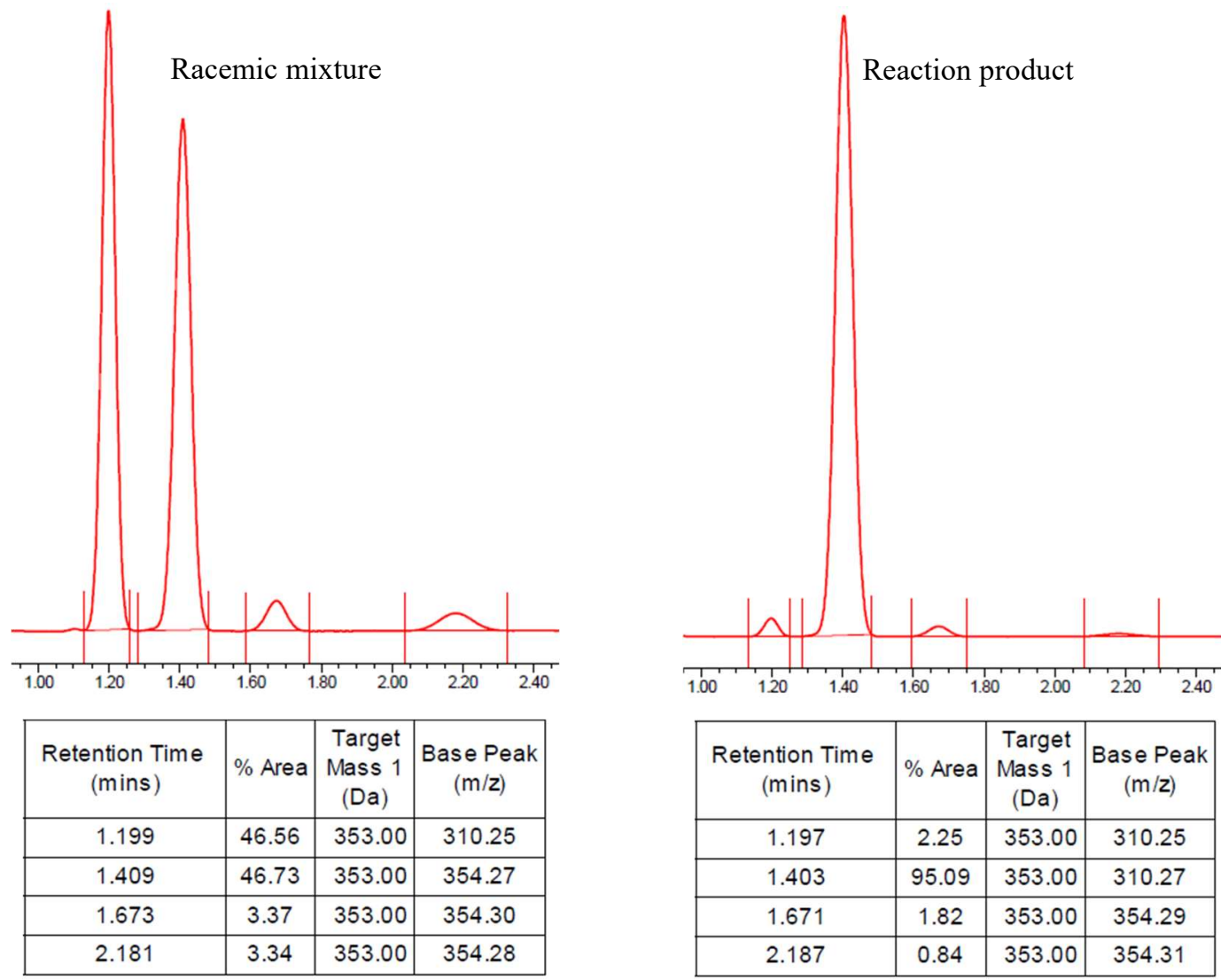
$\mathrm{OMe}$

1-benzyl 2-methyl (2R,3S)-3-(m-tolyl)pyrrolidine-1,2-dicarboxylate (10). The reaction was performed according to general procedure A with $m$-tolylboronic acid ( $82 \mathrm{mg}, 0.60 \mathrm{mmol}$, 1.2 equiv). The crude residue was purified by silica gel chromatography (0-40\% EtOAc in hexanes) to afford the title compound as clear colorless oil (135.8 mg, 77\% yield, 20:1 dr, 95\% ee). ${ }^{1} \mathbf{H}$ NMR $\left(600 \mathrm{MHz}, \mathrm{DMSO}-d_{6}\right) \delta 7.41(\mathrm{~s}, 2 \mathrm{H}), 7.40-7.37(\mathrm{~m}, 1 \mathrm{H}), 7.37-7.32$ (m, 1H), $7.37-7.23(\mathrm{~m}, 1 \mathrm{H}), 7.25-7.18(\mathrm{~m}, 1 \mathrm{H}), 7.13(\mathrm{~s}, 1 \mathrm{H}), 7.12-7.02(\mathrm{~m}, 2 \mathrm{H}), 5.24-5.09(\mathrm{~m}, 1.5 \mathrm{H})$, $5.01(\mathrm{~d}, J=12.7 \mathrm{~Hz}, 0.5 \mathrm{H}), 4.30(\mathrm{~d}, J=7.3 \mathrm{~Hz}, 0.5 \mathrm{H}), 4.28(\mathrm{~d}, J=7.1 \mathrm{~Hz}, 0.5 \mathrm{H}), 3.80-3.68(\mathrm{~m}, 1 \mathrm{H}), 3.62$ (s, 1.5H), $3.57-3.51(\mathrm{~m}, 1 \mathrm{H}), 3.50(\mathrm{~s}, 1.5 \mathrm{H}), 3.47-3.35(\mathrm{~m}, 1 \mathrm{H}), 2.30(\mathrm{~s}, 1.5 \mathrm{H}), 2.29(\mathrm{~s}, 1.5 \mathrm{H}), 2.27-$ $2.16(\mathrm{~m}, 1 \mathrm{H}), 2.13-1.97(\mathrm{~m}, 1 \mathrm{H}) .{ }^{13} \mathrm{C}$ NMR $\left(151 \mathrm{MHz}, \mathrm{DMSO}-d_{6}\right) \delta 172.3,171.9,153.8,153.3,140.3$, 140.2 , 137.77, 137.76, 136.8, 136.5, 128.5, 128.5, 128.4, 128.3, 127.82, 127.76, 127.74, 127.67, 127.5, 127.3, 124.11, 124.05, 66.3, 66.2, 65.6, 65.2, 51.83, 51.78, 49.5, 48.4, 46.6, 46.0, 32.7, 32.0, 20.94, 20.94. HRESIMS $354.1713 \mathrm{~m} / \mathrm{z}$ (calc'd for $\mathrm{C}_{21} \mathrm{H}_{24} \mathrm{NO}_{4}{ }^{+}, \mathrm{m} / \mathrm{z} 354.1705 \mathrm{~m} / \mathrm{z} ; \Delta \mathrm{ppm} 2.3$ )

Analysis of Stereochemistry: The racemic material was prepared according to general procedure C (16.6:1 dr). Absolute stereochemistry was assigned by analogy (see section V).

SFC-MS: Lux-4 4.6x100, $3.5 \mathrm{ml} / \mathrm{min}, 15 \% \mathrm{MeOH} w / 0.1 \% \mathrm{NH}_{4} \mathrm{OH}, 2000 \mathrm{psi}$
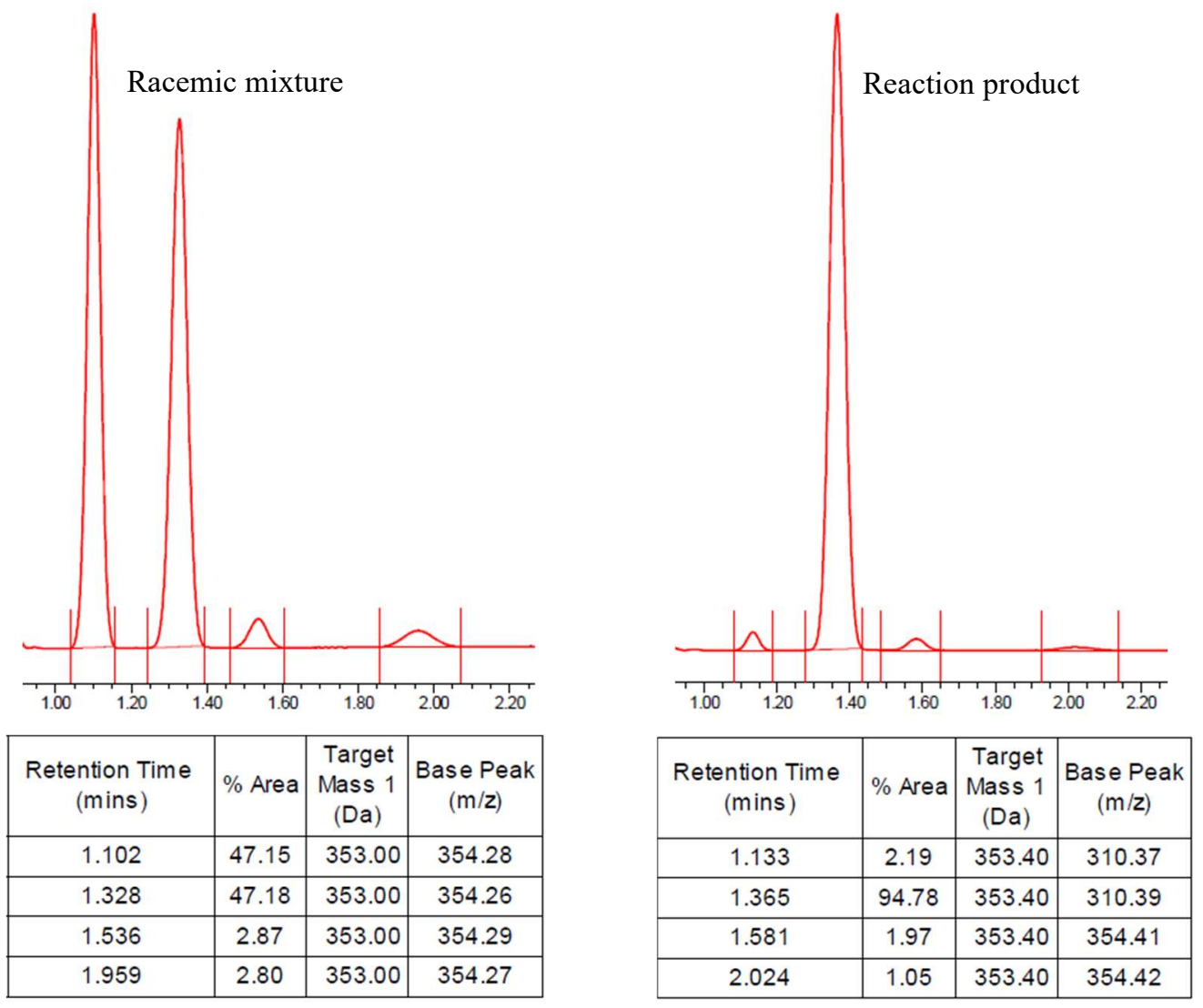


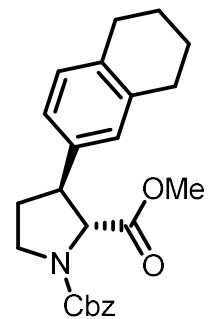

\section{1-benzyl 2-methyl $\quad(2 R, 3 S)-3-(5,6,7,8-t e t r a h y d r o n a p h t h a l e n-2-y l) p y r r o l i d i n e-1,2-$}

dicarboxylate (11). The reaction was performed according to general procedure A with (5,6,7,8-tetrahydronaphthalen-2-yl)boronic acid (132 mg, $0.75 \mathrm{mmol}, 1.5$ equiv). The crude residue was purified by silica gel chromatography (0-40\% EtOAc in hexanes) to afford the title compound as viscous clear colorless oil (153.1 mg, 78\% yield, $>20: 1 \mathrm{dr}, 95 \%$ ee). ${ }^{1} \mathbf{H}$ NMR $\left(600 \mathrm{MHz}, \mathrm{DMSO}-d_{6}\right) \delta 7.43-7.38(\mathrm{~m}, 2 \mathrm{H}), 7.36-7.32(\mathrm{~m}, 1 \mathrm{H}), 7.32-7.27(\mathrm{~m}, 2 \mathrm{H}), 7.01-6.96$ $(\mathrm{m}, 3 \mathrm{H}), 5.21-5.10(\mathrm{~m}, 1.5 \mathrm{H}), 5.00(\mathrm{~d}, J=12.8 \mathrm{~Hz}, 0.5 \mathrm{H}), 4.28(\mathrm{~d}, J=7.7 \mathrm{~Hz}, 0.5 \mathrm{H}), 4.27(\mathrm{~d}, J=7.8 \mathrm{~Hz}$, 0.5H), $3.76-3.67(\mathrm{~m}, 1 \mathrm{H}), 3.62(\mathrm{~s}, 1.5 \mathrm{H}), 3.57-3.52(\mathrm{~m}, 1 \mathrm{H}), 3.50(\mathrm{~s}, 1.5 \mathrm{H}), 3.43-3.37(\mathrm{~m}, 0.5 \mathrm{H}), 3.36$ - $3.32(\mathrm{~m}, 0.5 \mathrm{H}), 2.74-2.63(\mathrm{~m}, 4 \mathrm{H}), 2.41-2.12(\mathrm{~m}, 1 \mathrm{H}), 2.07-1.93(\mathrm{~m}, 1 \mathrm{H}), 1.75-1.63(\mathrm{~m}, 4 \mathrm{H}) .{ }^{13} \mathbf{C}$ NMR (151 MHz, DMSO-d $\left.{ }_{6}\right) \delta 172.3,172.0,153.8,153.3,137.3,137.2,136.8,136.7,136.5,135.4,129.12$, $129.09,128.3,128.2,127.8,127.7,127.44,127.40,127.3,124.1,124.0,66.3,66.2,65.7,65.2,51.8,51.7$, 49.3, 48.1, 46.6, 46.0, 32.7, 32.0, 28.8, 28.4, 22.73, 22.69. HRESIMS $394.2031 \mathrm{~m} / \mathrm{z}$ (calc'd for $\mathrm{C}_{24} \mathrm{H}_{28} \mathrm{NO}_{4}{ }^{+}, \mathrm{m} / \mathrm{z} 394.2018 \mathrm{~m} / \mathrm{z} ; \Delta \mathrm{ppm} 3.3$ )

Analysis of Stereochemistry: The racemic material was prepared according to general procedure C (15.3:1 dr). Absolute stereochemistry was assigned by analogy (see section V).

SFC-MS: Lux-4 4.6x100, $3.5 \mathrm{ml} / \mathrm{min}, 30 \% \mathrm{MeOH} w / 0.1 \% \mathrm{NH}_{4} \mathrm{OH}, 2000 \mathrm{psi}$
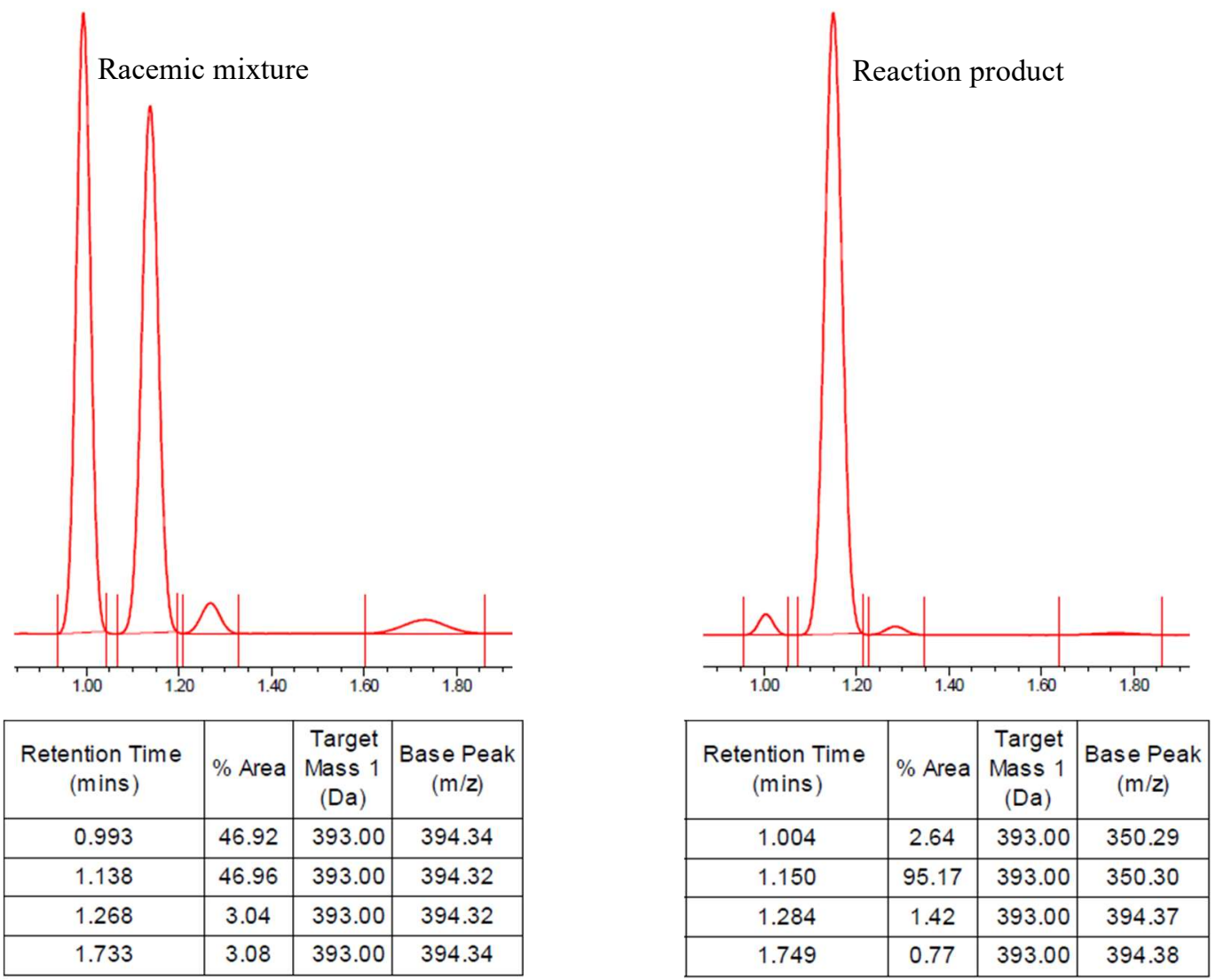

\begin{tabular}{|c|c|c|c|}
\hline $\begin{array}{c}\text { Retention Time } \\
\text { (mins) }\end{array}$ & \% Area & $\begin{array}{c}\text { Target } \\
\text { Mass 1 } \\
(\mathrm{Da})\end{array}$ & $\begin{array}{c}\text { Base Peak } \\
(\mathrm{m} / \mathbf{z})\end{array}$ \\
\hline 0.993 & 46.92 & 393.00 & 394.34 \\
\hline 1.138 & 46.96 & 393.00 & 394.32 \\
\hline 1.268 & 3.04 & 393.00 & 394.32 \\
\hline 1.733 & 3.08 & 393.00 & 394.34 \\
\hline
\end{tabular}

\begin{tabular}{|c|c|c|c|}
\hline $\begin{array}{c}\text { Retention Time } \\
(\mathrm{mins})\end{array}$ & \% Area & $\begin{array}{c}\text { Target } \\
\text { Mass 1 } \\
(\mathrm{Da})\end{array}$ & $\begin{array}{c}\text { Base Peak } \\
(\mathrm{m} / \mathbf{z})\end{array}$ \\
\hline 1.004 & 2.64 & 393.00 & 350.29 \\
\hline 1.150 & 95.17 & 393.00 & 350.30 \\
\hline 1.284 & 1.42 & 393.00 & 394.37 \\
\hline 1.749 & 0.77 & 393.00 & 394.38 \\
\hline
\end{tabular}




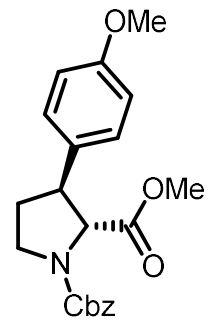

1-benzyl 2-methyl (2R,3S)-3-(4-methoxyphenyl)pyrrolidine-1,2-dicarboxylate (12). The reaction was performed according to general procedure A with (4-methoxyphenyl)boronic acid (114 mg, $0.75 \mathrm{mmol}, 1.5$ equiv). The crude residue was purified by silica gel chromatography (0-50\% EtOAc in hexanes) to afford the title compound as clear colorless oil (93.2 mg, 51\%, 19:1 dr, 96\% ee). ${ }^{1} \mathbf{H}$ NMR (600 MHz, DMSO-d $) \delta 7.52-7.38(\mathrm{~m}, 2 \mathrm{H})$, $7.35(\mathrm{~d}, J=7.5 \mathrm{~Hz}, 1 \mathrm{H}), 7.31-7.26(\mathrm{~m}, 2 \mathrm{H}), 7.22(\mathrm{~d}, J=8.5 \mathrm{~Hz}, 2 \mathrm{H}), 6.90(\mathrm{~d}, J=5.9 \mathrm{~Hz}, 1 \mathrm{H}), 6.88(\mathrm{~d}, J$ $=5.6 \mathrm{~Hz}, 1 \mathrm{H}), 5.19-5.07(\mathrm{~m}, 1.5 \mathrm{H}), 4.99(\mathrm{~d}, J=12.7 \mathrm{~Hz}, 0.5 \mathrm{H}), 4.23(\mathrm{~d}, J=7.5 \mathrm{~Hz}, 0.5 \mathrm{H}), 4.20(\mathrm{~d}, J=$ $7.4 \mathrm{~Hz}, 0.5 \mathrm{H}), 3.73(\mathrm{~s}, 1.5 \mathrm{H}), 3.73(\mathrm{~s}, 1.5 \mathrm{H}), 3.70(\mathrm{~m}, 1 \mathrm{H}), 3.61(\mathrm{~s}, 1.5 \mathrm{H}), 3.56-3.49(\mathrm{~m}, 1 \mathrm{H}), 3.48(\mathrm{~s}$, $1.5 \mathrm{H}), 3.45-3.33(\mathrm{~m}, 1 \mathrm{H}), 2.26-2.13(\mathrm{~m}, 1 \mathrm{H}), 2.10-1.97(\mathrm{~m}, 1 \mathrm{H}) .{ }^{13} \mathbf{C}$ NMR $(151 \mathrm{MHz}, \mathrm{DMSO}) \delta$ $172.3,172.0,158.3,153.8,153.3,136.8,136.5,132.0,131.9,128.4,128.3,128.2,128.1,127.83,127.75$, $127.5,127.3,113.98,113.96,66.3,66.2,65.8,65.4,55.0,51.82,51.77,48.9,47.8,46.6,46.0,32.8,32.1$.

HRESIMS $370.1653 \mathrm{~m} / \mathrm{z}$ (calc'd for $\mathrm{C}_{21} \mathrm{H}_{24} \mathrm{NO}_{4}{ }^{+}, \mathrm{m} / \mathrm{z} 370.1654 \mathrm{~m} / \mathrm{z} ; \Delta \mathrm{ppm} 0.1$ )

Analysis of Stereochemistry: The racemic material was prepared according to general procedure C (15.1:1 dr). Absolute stereochemistry was assigned by analogy (see section V).

SFC-MS: Lux-4 4.6x100, $3.5 \mathrm{ml} / \mathrm{min}, 15 \% \mathrm{MeOH} w / 0.1 \% \mathrm{NH}_{4} \mathrm{OH}, 2000 \mathrm{psi}$
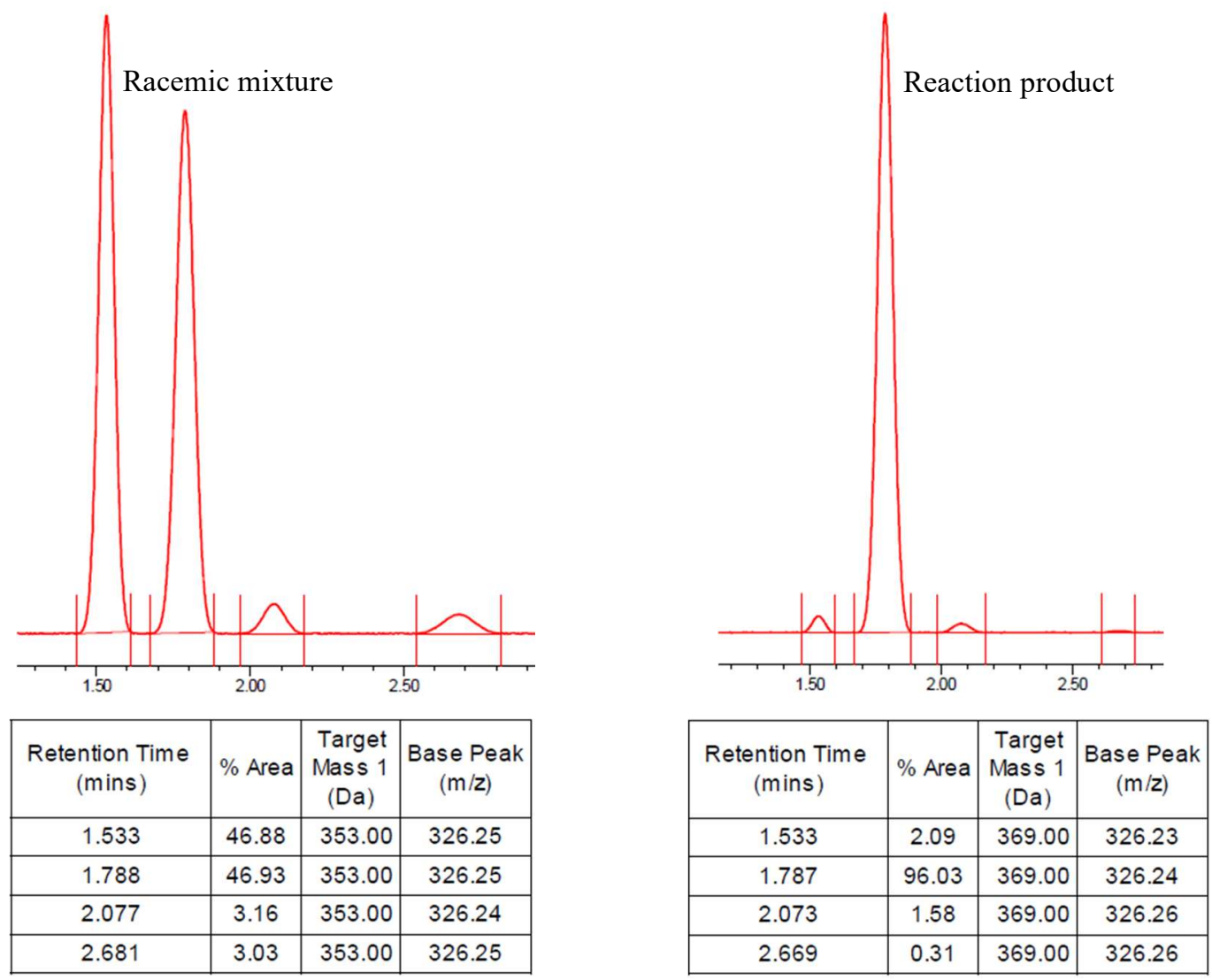

\begin{tabular}{|c|c|c|c|}
\hline $\begin{array}{c}\text { Retention Time } \\
(\mathrm{mins})\end{array}$ & \% Area & $\begin{array}{c}\text { Target } \\
\text { Mass 1 } \\
(\mathrm{Da})\end{array}$ & $\begin{array}{c}\text { Base Peak } \\
(\mathrm{m} / \mathrm{z})\end{array}$ \\
\hline 1.533 & 46.88 & 353.00 & 326.25 \\
\hline 1.788 & 46.93 & 353.00 & 326.25 \\
\hline 2.077 & 3.16 & 353.00 & 326.24 \\
\hline 2.681 & 3.03 & 353.00 & 326.25 \\
\hline
\end{tabular}

\begin{tabular}{|c|c|c|c|}
\hline $\begin{array}{c}\text { Retention Time } \\
(\mathrm{mins})\end{array}$ & \% Area & $\begin{array}{c}\text { Target } \\
\text { Mass 1 } \\
(\mathrm{Da})\end{array}$ & $\begin{array}{c}\text { Base Peak } \\
(\mathrm{m} / \mathrm{z})\end{array}$ \\
\hline 1.533 & 2.09 & 369.00 & 326.23 \\
\hline 1.787 & 96.03 & 369.00 & 326.24 \\
\hline 2.073 & 1.58 & 369.00 & 326.26 \\
\hline 2.669 & 0.31 & 369.00 & 326.26 \\
\hline
\end{tabular}




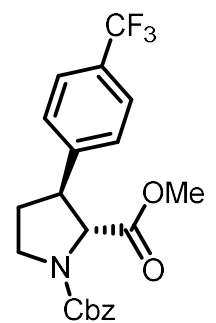

\section{1-benzyl 2-methyl (2R,3S)-3-(4-(trifluoromethyl)phenyl)pyrrolidine-1,2-dicarboxylate} (13). The reaction was performed according to general procedure A with (4trifluoromethylphenyl)boronic acid (142 $\mathrm{mg}, 0.75 \mathrm{mmol}, 1.5$ equiv). The crude residue was purified by silica gel chromatography $(0-50 \%$ EtOAc in hexanes) to afford the title compound as clear colorless oil (162.1 mg, 80\%, 14:1 dr, 93\% ee). ${ }^{1} \mathbf{H}$ NMR (600 MHz, DMSO- $\left.d_{6}\right) \delta 7.69(\mathrm{~d}, J=7.4,1 \mathrm{H}), 7.67(\mathrm{~d}, J=7.4,1 \mathrm{H}), 7.57(\mathrm{~s}, 1 \mathrm{H}), 7.56(\mathrm{~s}, 1 \mathrm{H}), 7.44-7.37(\mathrm{~m}, 2 \mathrm{H})$, $7.36-7.32(\mathrm{~m}, 1 \mathrm{H}), 7.31-7.27(\mathrm{~m}, 2 \mathrm{H}), 5.19-5.10(\mathrm{~m}, 1.5 \mathrm{H}), 5.00(\mathrm{~d}, J=12.8 \mathrm{~Hz}, 0.5 \mathrm{H}), 4.37(\mathrm{~d}, J=$ $7.4 \mathrm{~Hz}, 0.5 \mathrm{H}), 4.34(\mathrm{~d}, J=7.3 \mathrm{~Hz}, 0.5 \mathrm{H}), 3.74(\mathrm{~m}, 1.5 \mathrm{H}), 3.62(\mathrm{~s}, 1.5 \mathrm{H}), 3.61-3.57(\mathrm{~m}, 0.5 \mathrm{H}), 3.56-3.52$ (m, 1H), $3.50(\mathrm{~s}, 1.5 \mathrm{H}), 2.33-2.23(\mathrm{~m}, 1 \mathrm{H}), 2.15-2.05(\mathrm{~m}, 1 \mathrm{H}) .{ }^{13} \mathbf{C}$ NMR (151 MHz, DMSO-d 6 ) $\delta$ 172.0, 171.6, 153.8, 153.3, 145.1, 145.0, 136.7, 136.5, 128.4, 128.2, 128.13, 12809, 127.8, 127.8, 127.5, 127.4, $125.5,125.43,125.40,66.4,66.3,65.3,64.9,51.91,51.86,49.13,48.05,46.6,46.0,32.6,31.9 .{ }^{19} \mathbf{F}$ NMR (564 MHz, DMSO- $d_{6}$ ) 60.91, 60.92. HRESIMS $408.1430 \mathrm{~m} / \mathrm{z}$ (calc' $\mathrm{d}$ for $\mathrm{C}_{21} \mathrm{H}_{21} \mathrm{NO}_{4} \mathrm{~F}_{3}{ }^{+}, \mathrm{m} / \mathrm{z} 408.1423$ $\mathrm{m} / \mathrm{z} ; \Delta \mathrm{ppm} 2.2)$

Analysis of Stereochemistry: The racemic material was prepared according to general procedure C (14.7:1 dr). Absolute stereochemistry was assigned by analogy (see section V).

SFC-MS: Lux-4 4.6x100, $3.5 \mathrm{ml} / \mathrm{min}, 10 \% \mathrm{MeOH} w / 0.1 \% \mathrm{NH}_{4} \mathrm{OH}, 2000 \mathrm{psi}$
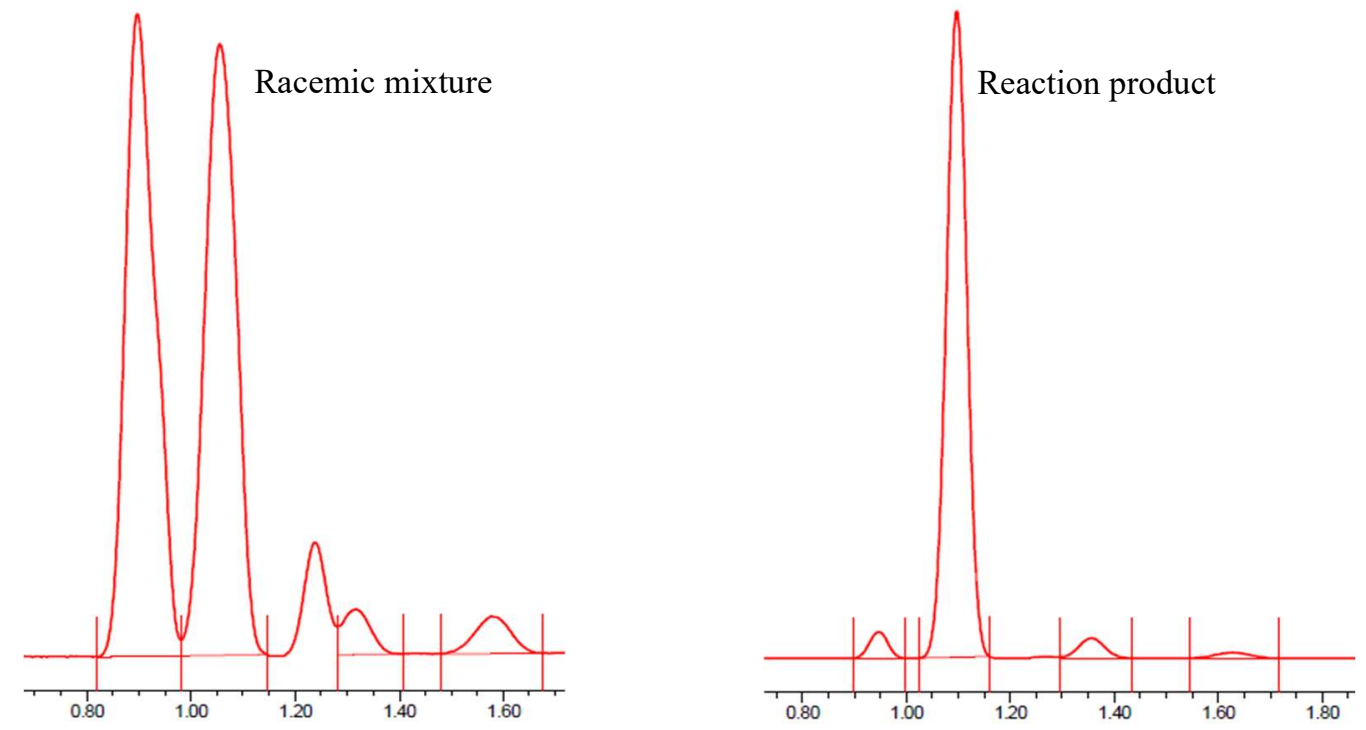

\begin{tabular}{|c|c|c|c|}
\hline $\begin{array}{c}\text { Retention Time } \\
(\mathrm{mins})\end{array}$ & \% Area & $\begin{array}{c}\text { Target } \\
\text { Mass 1 } \\
(\mathrm{Da})\end{array}$ & $\begin{array}{c}\text { Base Peak } \\
(\mathrm{m} / \mathrm{z})\end{array}$ \\
\hline 0.897 & 46.76 & 407.00 & 364.28 \\
\hline 1.055 & 46.88 & 407.00 & 364.27 \\
\hline 1.317 & 3.08 & 407.00 & 408.30 \\
\hline 1.578 & 3.28 & 407.00 & 408.28 \\
\hline
\end{tabular}

\begin{tabular}{|c|c|c|c|}
\hline $\begin{array}{c}\text { Retention Time } \\
(\mathrm{mins})\end{array}$ & \% Area & $\begin{array}{c}\text { Target } \\
\text { Mass 1 } \\
(\mathrm{Da})\end{array}$ & $\begin{array}{c}\text { Base Peak } \\
(\mathrm{m} / \mathbf{z})\end{array}$ \\
\hline 0.946 & 3.36 & 407.40 & 364.41 \\
\hline 1.097 & 91.70 & 407.40 & 364.41 \\
\hline 1.357 & 3.59 & 407.40 & 408.46 \\
\hline 1.627 & 1.35 & 407.40 & 408.47 \\
\hline
\end{tabular}




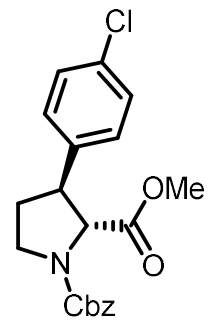

1-benzyl 2-methyl (2R,3S)-3-(4-chlorophenyl)pyrrolidine-1,2-dicarboxylate (14). The reaction was performed according to general procedure A with (4-chlorophenyl)boronic acid (117 mg, $0.75 \mathrm{mmol}, 1.5$ equiv). The crude residue was purified by silica gel chromatography $(0-50 \%$ EtOAc in hexanes) to afford the title compound as clear colorless oil (143 mg, 77\% yield, 18:1 dr, 96\% ee). ${ }^{1} \mathbf{H}$ NMR (600 MHz, DMSO-d $\left.d_{6}\right) \delta 7.53-7.43(\mathrm{~m}$, 1H), $7.41-7.37(\mathrm{~m}, 2 \mathrm{H}), 7.36-7.31(\mathrm{~m}, 3 \mathrm{H}), 7.31-7.26(\mathrm{~m}, 2 \mathrm{H}), 7.21-7.13(\mathrm{~m}, 1 \mathrm{H}), 5.17$ - $5.09(\mathrm{~m}$, $1.5 \mathrm{H}), 4.98(\mathrm{~d}, J=12.8 \mathrm{~Hz}, 0.5 \mathrm{H}), 4.30(\mathrm{~d}, J=7.8 \mathrm{~Hz}, 0.5 \mathrm{H}), 4.26(\mathrm{~d}, J=7.7 \mathrm{~Hz}, 0.5 \mathrm{H}), 3.78-3.69(\mathrm{~m}$, 1H), $3.61(\mathrm{~s}, 1.5 \mathrm{H}), 3.49(\mathrm{~s}, 1.5 \mathrm{H}), 3.49-3.41(\mathrm{~m}, 2 \mathrm{H}), 2.29-2.16(\mathrm{~m}, 1 \mathrm{H}), 2.16-2.00(\mathrm{~m}, 1 \mathrm{H}) .{ }^{13} \mathbf{C ~ N M R}$ $\left(151 \mathrm{MHz}, \mathrm{DMSO}-d_{6}\right) \delta 171.7,171.4,153.7,153.2,150.14,150.05,149.3,149.2,148.5,148.4,147.64$, 147.56, 136.6, 136.3, 128.2, 128.1, 127.6, 127.24, 127.19, 123.9, 117.3, 117.2, 116.2, 116.1, 66.2, 66.1, 65.3, 64.9, 51.6, 50.9, 48.5, 47.4, 46.3, 45.8, 32.4, 31.7. HRESIMS $396.0993 \mathrm{~m} / \mathrm{z}$ (calc'd for $\mathrm{C}_{20} \mathrm{H}_{20} \mathrm{ClNO}_{4} \mathrm{Na}^{+}, \mathrm{m} / \mathrm{z} 396.0979 \mathrm{~m} / \mathrm{z} ; \Delta \mathrm{ppm} 3.5$ )

Analysis of Stereochemistry: The racemic material was prepared according to general procedure C (18.7:1 dr). Absolute stereochemistry was assigned by analogy (see section V).

SFC-MS: Lux-4 4.6x100, $3.5 \mathrm{ml} / \mathrm{min}, 15 \% \mathrm{MeOH} w / 0.1 \% \mathrm{NH}_{4} \mathrm{OH}, 2000 \mathrm{psi}$

Racemic mixture

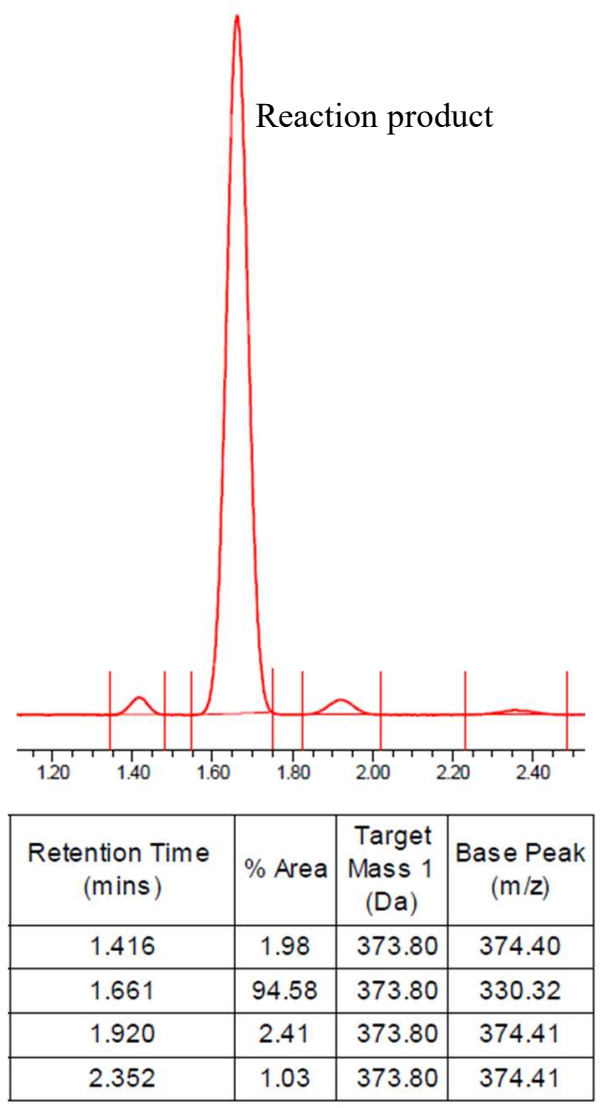




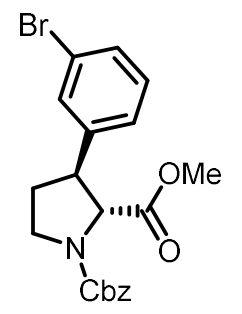

\section{1-benzyl 2-methyl (2R,3S)-3-(3-bromophenyl)pyrrolidine-1,2-dicarboxylate (15).}

The reaction was performed according to general procedure A with (3bromophenyl)boronic acid ( $151 \mathrm{mg}, 0.75 \mathrm{mmol}, 1.5$ equiv). The crude residue was purified by silica gel chromatography (0-35\% EtOAc in hexanes) then purified by mass directed reverse phase HPLC with TFA modifier to afford the title compound as clear colorless oil (143 mg, 68\% yield, 19:1 dr, 96\% ee). ${ }^{1}$ H NMR (600 MHz, DMSO- $\left.d_{6}\right) \delta 7.58(\mathrm{~s}, 1 \mathrm{H}), 7.45$ (d, $J=8.6 \mathrm{~Hz}$, 1H), $7.39(\mathrm{~s}, 1 \mathrm{H}), 7.37-7.31(\mathrm{~m}, 2 \mathrm{H}), 7.31-7.23(\mathrm{~m}, 4 \mathrm{H}), 5.19-5.10(\mathrm{~m}, 1.5 \mathrm{H}), 5.00(\mathrm{~d}, J=12.7 \mathrm{~Hz}$, $0.5 \mathrm{H}), 4.32(\mathrm{~d}, J=7.6 \mathrm{~Hz}, 0.5 \mathrm{H}), 4.29$ (d, $J=7.5 \mathrm{~Hz}, 0.5 \mathrm{H}), 3.72(\mathrm{tdd}, J=10.5,7.8,3.3 \mathrm{~Hz}, 1 \mathrm{H}), 3.62(\mathrm{~s}$, 1.5H), 3.49 (s, 1.5H), $3.48-3.40(\mathrm{~m}, 2 \mathrm{H}), 2.31-2.16(\mathrm{~m}, 1 \mathrm{H}), 2.13-1.96(\mathrm{~m}, 1 \mathrm{H}) .{ }^{13} \mathbf{C ~ N M R}(151 \mathrm{MHz}$, DMSO-d $\left.{ }_{6}\right) \delta 172.0,171.6,153.8,153.3,143.1,142.9,136.7,136.4,130.69,130.66,130.0,128.3,128.2$, $127.8,127.7,127.4,127.3,126.3,126.2,121.9,66.3,66.2,65.4,64.9,51.9,51.8,49.0,47.9,46.6,46.0$, 32.7, 32.0. HRESIMS $418.0662 \mathrm{~m} / \mathrm{z}$ (calc'd for $\mathrm{C}_{20} \mathrm{H}_{21} \mathrm{BrNO}_{4}{ }^{+}, \mathrm{m} / \mathrm{z} 418.0654 \mathrm{~m} / \mathrm{z} ; \Delta \mathrm{ppm} 1.9$ )

Analysis of Stereochemistry: The racemic material was prepared according to general procedure C (15:1 dr). Absolute stereochemistry was assigned by analogy (see section V).

SFC-MS: Lux-4 4.6x100, $3.5 \mathrm{ml} / \mathrm{min}, 20 \% \mathrm{MeOH} w / 0.1 \% \mathrm{NH}_{4} \mathrm{OH}, 2000 \mathrm{psi}$
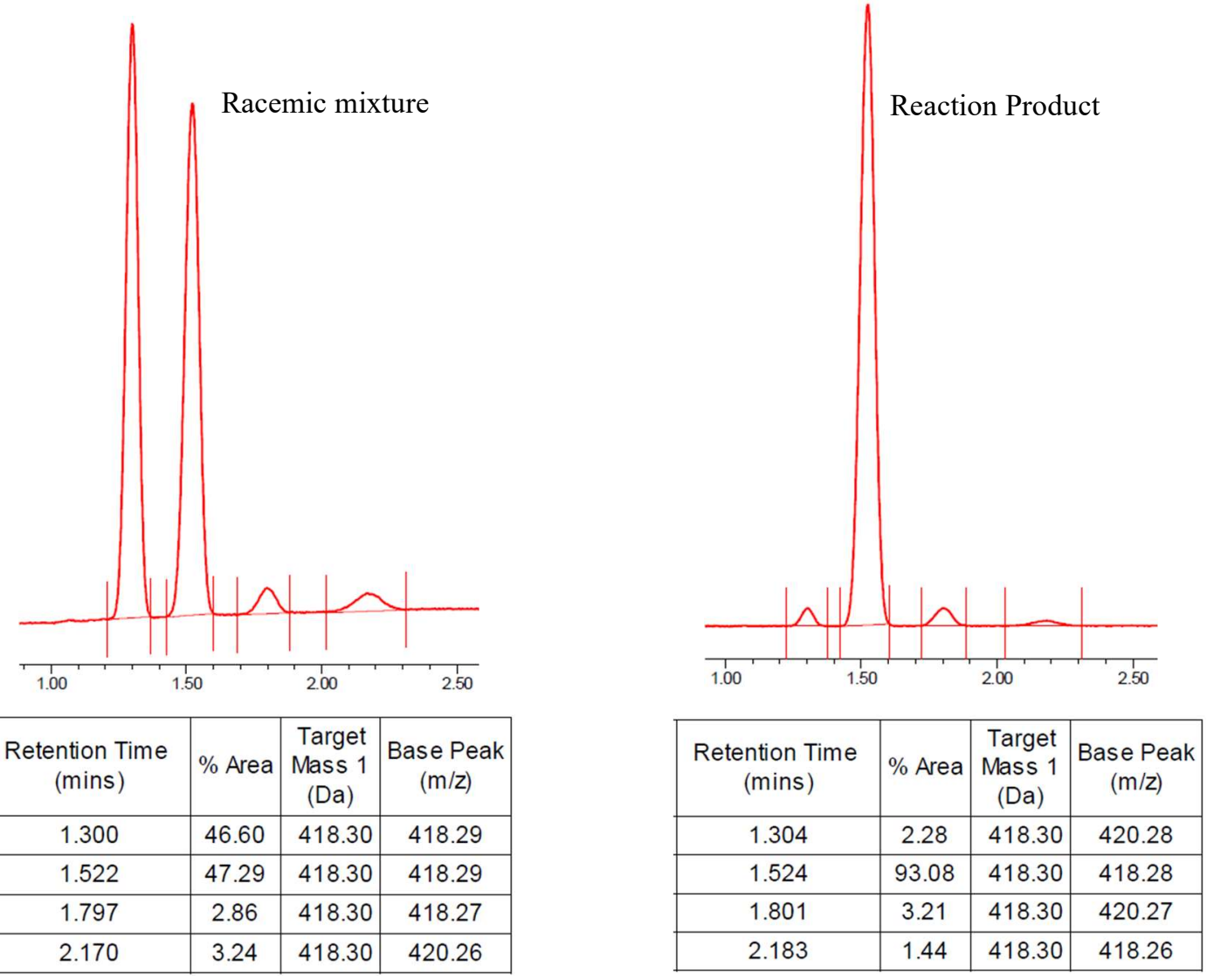


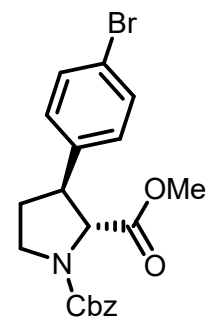

\section{1-benzyl 2-methyl (2R,3S)-3-(4-bromophenyl)pyrrolidine-1,2-dicarboxylate (16).}

The reaction was performed according to general procedure A with (4-bromophenyl)boronic acid (151 mg, $0.75 \mathrm{mmol}, 1.5$ equiv). The crude residue was purified by silica gel chromatography $(0-50 \%$ EtOAc in hexanes) to afford the title compound as clear colorless oil (154 mg, 74\% yield, 16:1 dr, 96\% ee). ${ }^{1} \mathbf{H}$ NMR (600 MHz, DMSO- $\left.d_{6}\right) \delta 7.52(\mathrm{~d}, J=5.1$ $\mathrm{Hz}, 1 \mathrm{H}), 7.50(\mathrm{~d}, J=5.3 \mathrm{~Hz}, 1 \mathrm{H}), 7.42-7.36(\mathrm{~m}, 2 \mathrm{H}), 7.35-7.33(\mathrm{~m}, 1 \mathrm{H}), 7.32-7.23(\mathrm{~m}, 3 \mathrm{H}), 5.25-$ $5.07(\mathrm{~m}, 1.5 \mathrm{H}), 4.99(\mathrm{~d}, J=12.7 \mathrm{~Hz}, 0.5 \mathrm{H}), 4.27(\mathrm{~d}, J=7.4 \mathrm{~Hz}, 0.5 \mathrm{H}), 4.25(\mathrm{~d}, J=7.4 \mathrm{~Hz}, 0.5 \mathrm{H}), 3.75-$ $3.67(\mathrm{~m}, 1 \mathrm{H}), 3.61(\mathrm{~s}, 1.5 \mathrm{H}), 3.56-3.51(\mathrm{~m}, 1 \mathrm{H}), 3.51(\mathrm{~s}, 1 \mathrm{H}), 3.49(\mathrm{~s}, 1.5 \mathrm{H}), 3.47-3.39(\mathrm{~m}, 1 \mathrm{H}), 2.30-$ $2.15(\mathrm{~m}, 1 \mathrm{H}), 2.09-1.91(\mathrm{~m}, 1 \mathrm{H}) .{ }^{13} \mathbf{C}$ NMR $\left(151 \mathrm{MHz}, \mathrm{DMSO}-d_{6}\right) \delta 172.0,171.7,153.8,153.3,139.7$, $139.6,136.7,136.4,131.5,131.4,129.44,129.41,128.4,128.3,128.2,127.82$, 127.77, 127.75, 127.5, 127.3, 120.2, 66.3, 66.2, 65.4, 65.0, 51.90, 51.85, 48.9, 47.8, 46.5, 46.0, 32.6, 31.9. HRESIMS 418.0662 $\mathrm{m} / \mathrm{z}$ (calc'd for $\mathrm{C}_{20} \mathrm{H}_{21} \mathrm{BrNO}_{4}^{+}, \mathrm{m} / \mathrm{z} 418.0654 \mathrm{~m} / \mathrm{z} ; \Delta \mathrm{ppm}$ 2.2)

Analysis of Stereochemistry: The racemic material was prepared according to general procedure C (13:1 dr). Absolute stereochemistry was assigned by analogy (see section V).

SFC-MS: Lux-4 4.6x100, $3.5 \mathrm{ml} / \mathrm{min}, 15 \% \mathrm{MeOH} w / 0.1 \% \mathrm{NH}_{4} \mathrm{OH}, 2000 \mathrm{psi}$

Racemic mixture

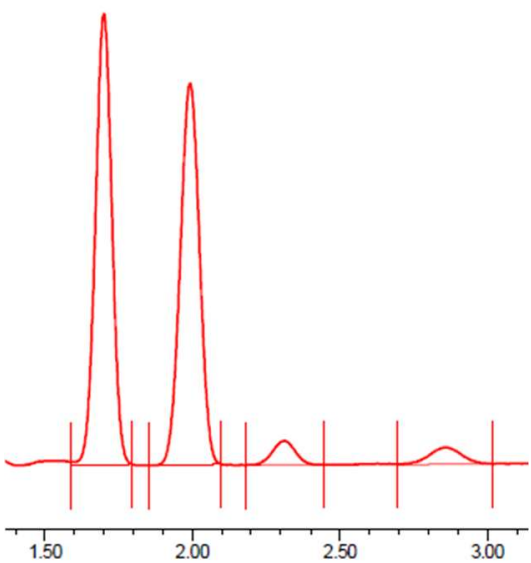

\begin{tabular}{|c|c|c|c|}
\hline $\begin{array}{c}\text { Retention Time } \\
(\mathrm{mins})\end{array}$ & $\%$ Area & $\begin{array}{c}\text { Target } \\
\text { Mass 1 } \\
(\mathrm{Da})\end{array}$ & $\begin{array}{c}\text { Base Peak } \\
(\mathrm{m} / \mathbf{z})\end{array}$ \\
\hline 1.700 & 46.94 & 419.00 & 420.22 \\
\hline 1.992 & 46.19 & 419.00 & 420.21 \\
\hline 2.312 & 3.45 & 419.00 & 420.21 \\
\hline 2.859 & 3.41 & 419.00 & 420.18 \\
\hline
\end{tabular}

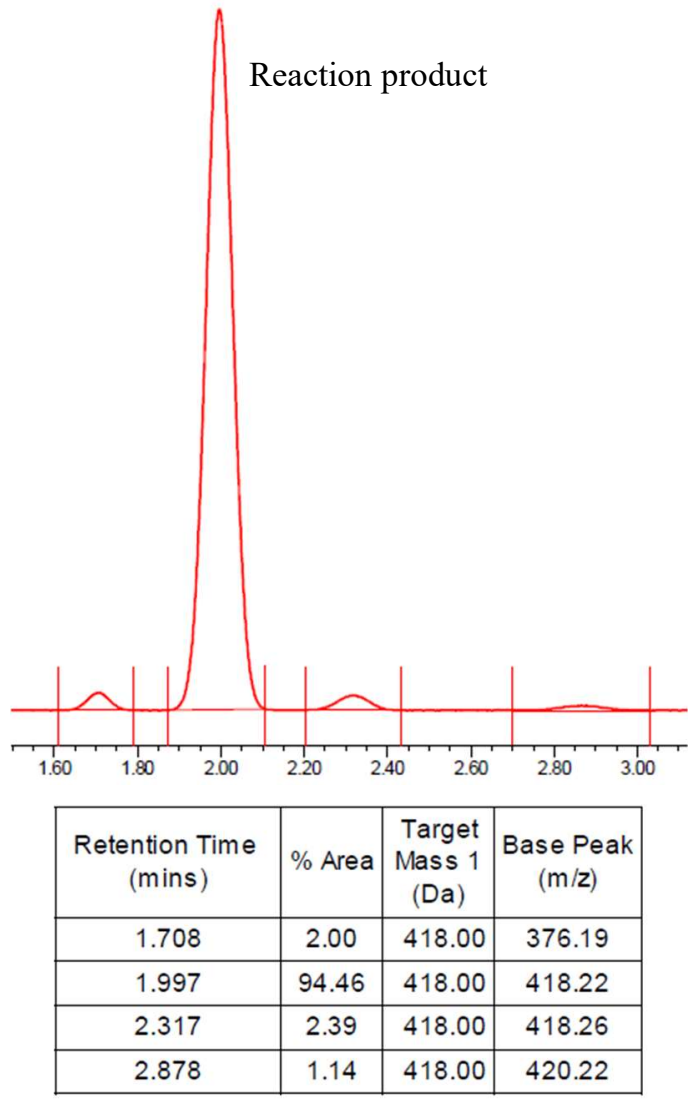




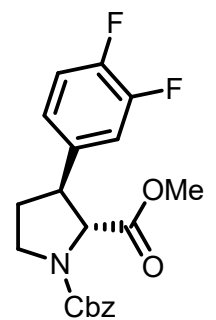

1-benzyl 2-methyl (2R,3S)-3-(3,4-difluorophenyl)pyrrolidine-1,2-dicarboxylate (17). The reaction was performed according to general procedure A with (3,4-difluorophenyl)boronic acid (118 mg, $0.75 \mathrm{mmol}, 1.5$ equiv). The crude residue was purified by silica gel chromatography $(0-50 \%$ EtOAc in hexanes) to afford the title compound and starting material 1 as clear colorless oil (134 mg, $81 \mathrm{wt} \% \mathbf{1 6}, 58 \%$ yield). This mixture was then purified by mass directed reverse phase HPLC with TFA modifier to afford the title compound as clear colorless oil (91.7 mg, 49\% yield, >20:1 dr, 96\% ee). ${ }^{1} \mathbf{H}$ NMR (600 MHz, DMSO- $\left.d_{6}\right) \delta 7.40-7.36(\mathrm{~m}$, $3.5 \mathrm{H}), 7.34(\mathrm{~d}, J=8.6 \mathrm{~Hz}, 3.5 \mathrm{H}), 7.32-7.26(\mathrm{~m}, 1 \mathrm{H}), 5.16-5.09(\mathrm{~m}, 1.5 \mathrm{H}), 4.98(\mathrm{~d}, J=12.7 \mathrm{~Hz}, 0.5 \mathrm{H})$, $4.27(\mathrm{~d}, J=7.5 \mathrm{~Hz}, 0.5 \mathrm{H}), 4.24$ (d, $J=7.4 \mathrm{~Hz}, 0.5 \mathrm{H}), 3.71$ (ddd, $J=11.0,7.9,3.3 \mathrm{~Hz}, 1 \mathrm{H}), 3.61(\mathrm{~s}, 1.5 \mathrm{H})$, $3.55-3.50(\mathrm{~m}, 1 \mathrm{H}), 3.49(\mathrm{~s}, 1.5 \mathrm{H}), 3.48-3.41(\mathrm{~m}, 1 \mathrm{H}), 2.31-2.14(\mathrm{~m}, 1 \mathrm{H}), 2.12-1.97(\mathrm{~m}, 1 \mathrm{H}) .{ }^{13} \mathbf{C}$ NMR $\left(151 \mathrm{MHz}, \mathrm{DMSO}-d_{6}\right) \delta 172.0,171.7,153.8,153.3,139.2,139.1,136.7,136.4,131.7,129.10$, 129.07, 128.54, 128.51, 128.4, 128.2, 127.83, 127.75, 127.5, 127.3, 66.3, 66.2, 65.5, 65.0, 51.9, 51.8, 48.8, 47.7, 46.5, 46.0, 32.6, 31.9. ${ }^{19}$ F NMR (564 MHz, DMSO-d $)$ - 138.3 - -138.4 (m, 1F), -140.8 - -140.9 (m, 1F). HRESIMS $398.1193 \mathrm{~m} / \mathrm{z}$ (calc'd for $\mathrm{C}_{20} \mathrm{H}_{19} \mathrm{NO}_{4} \mathrm{~F}_{2} \mathrm{Na}^{+}, \mathrm{m} / \mathrm{z} 398.1180 \mathrm{~m} / \mathrm{z} ; \Delta \mathrm{ppm} 3.3$ )

Analysis of Stereochemistry: The racemic material was prepared according to general procedure C ( $>20: 1$ dr). Absolute stereochemistry was assigned by analogy (see section V).

SFC-MS: Lux-4 4.6x100, $3.5 \mathrm{ml} / \mathrm{min}, 10 \% \mathrm{MeOH}$ w/ 0.1\% $\mathrm{NH}_{4} \mathrm{OH}, 2000 \mathrm{psi}$
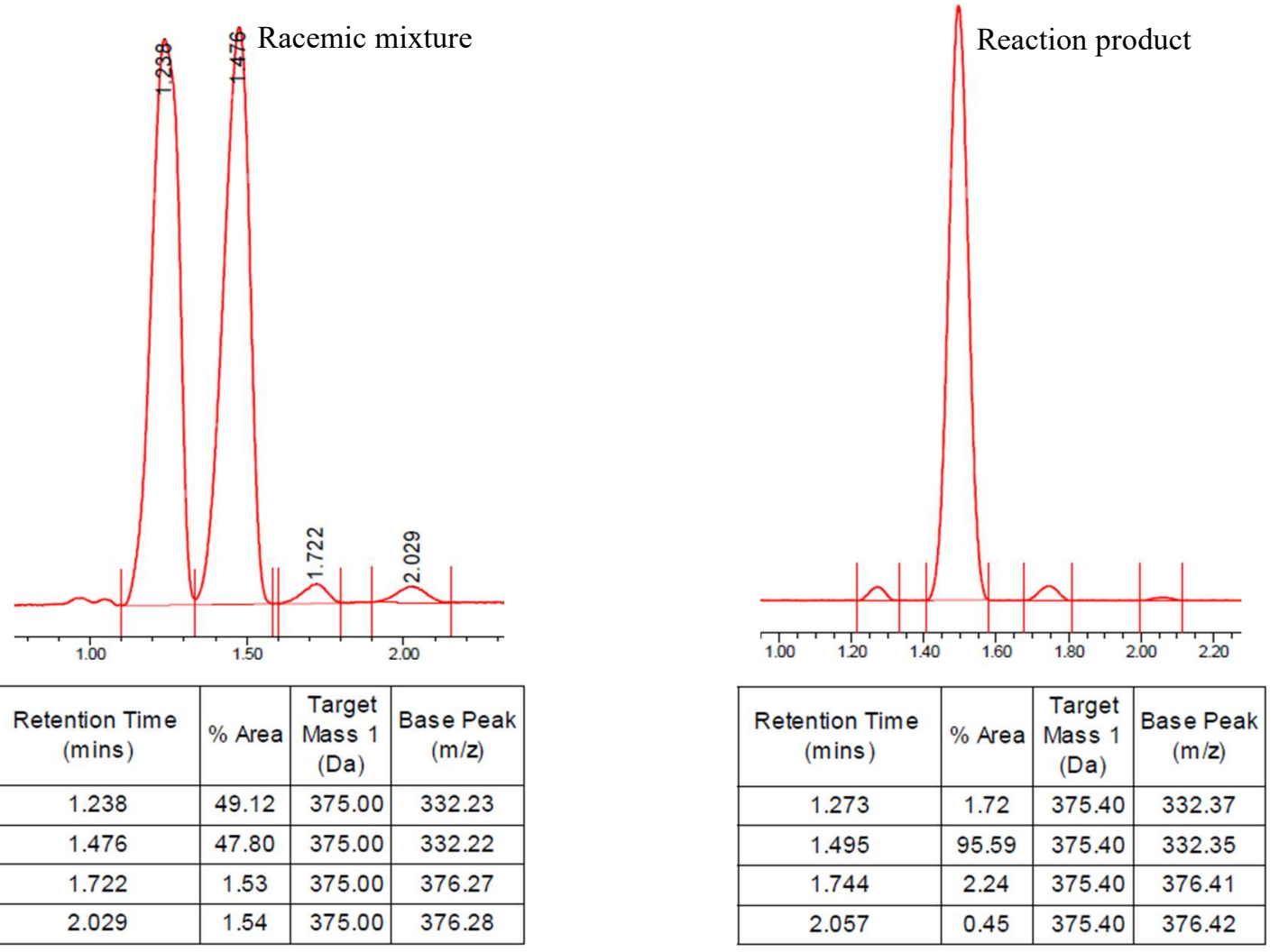


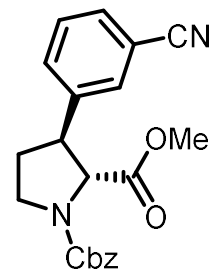

1-benzyl 2-methyl (2R,3S)-3-(3-cyanophenyl)pyrrolidine-1,2-dicarboxylate (18). The reaction was performed according to general procedure A with (3-cyanophenyl)boronic acid (110 mg, $0.75 \mathrm{mmol}, 1.5$ equiv). The crude residue was purified by silica gel chromatography (0-80\% EtOAc in hexanes) to afford the title compound as clear colorless oil (85.6 mg, 47\% yield, 14:1 dr, 93\% ee). ${ }^{1}$ H NMR (600 MHz, DMSO- $\left.d_{6}\right) \delta 7.87(\mathrm{~s}, 1 \mathrm{H})$, $7.74(\mathrm{~d}, J=3.2 \mathrm{~Hz}, 0.5 \mathrm{H}), 7.72(\mathrm{~d}, J=3.1 \mathrm{~Hz}, 0.5 \mathrm{H}), 7.70(\mathrm{~s}, 0.5 \mathrm{H}), 7.68(\mathrm{~s}, 0.5 \mathrm{H}), 7.55(\mathrm{t}, J=7.2 \mathrm{~Hz}$, $0.5 \mathrm{H}), 7.54(\mathrm{~d}, J=7.4 \mathrm{~Hz}, 0.5 \mathrm{H}), 7.41-7.37(\mathrm{~m}, 2 \mathrm{H}), 7.37-7.32(\mathrm{~m}, 1.5 \mathrm{H}), 7.31-7.26(\mathrm{~m}, 1.5 \mathrm{H}), 5.16$ $-5.09(\mathrm{~m}, 1.5 \mathrm{H}), 4.98(\mathrm{~d}, J=12.7 \mathrm{~Hz}, 0.5 \mathrm{H}), 4.35(\mathrm{~d}, J=7.7 \mathrm{~Hz}, 0.5 \mathrm{H}), 4.31(\mathrm{~d}, J=7.6 \mathrm{~Hz}, 0.5 \mathrm{H}), 3.74$ (ddd, $J=10.7,7.8,3.1 \mathrm{~Hz}, 1 \mathrm{H}), 3.61(\mathrm{~s}, 1.5 \mathrm{H}), 3.58-3.43(\mathrm{~m}, 1.5 \mathrm{H}), 3.49(\mathrm{~s}, 2 \mathrm{H}), 2.31-2.20(\mathrm{~m}, 1 \mathrm{H})$, $2.18-2.03(\mathrm{~m}, 1 \mathrm{H}) .{ }^{13} \mathbf{C}$ NMR $\left(151 \mathrm{MHz}, \mathrm{DMSO}-d_{6}\right) \delta 171.9,171.5,153.8,153.3,141.9,141.8,136.7$, 136.4, 132.43, 132.37, 131.1, 131.03, 130.98, 129.81, 129.78, 128.4, 128.3, 127.83, 127.76, 127.5, 127.3, 118.6, 111.7, 111.6, 66.3, 66.2, 65.3, 64.8, 52.0, 51.9, 48.9, 47.8, 46.6, 46.1, 32.6, 32.0. HRESIMS $365.1484 \mathrm{~m} / \mathrm{z}$ (calc'd for $\mathrm{C}_{21} \mathrm{H}_{21} \mathrm{~N}_{2} \mathrm{O}_{4}{ }^{+}, \mathrm{m} / \mathrm{z} 365.1501 \mathrm{~m} / \mathrm{z} ; \Delta \mathrm{ppm} 2.7$ )

Analysis of Stereochemistry: The racemic material was prepared according to general procedure C $(>20: 1$ dr). Absolute stereochemistry was assigned by analogy (see section V).

SFC-MS: Lux-4 4.6x100, $3.5 \mathrm{ml} / \mathrm{min}, 25 \% \mathrm{MeOH} w / 0.1 \% \mathrm{NH}_{4} \mathrm{OH}, 2000 \mathrm{psi}$

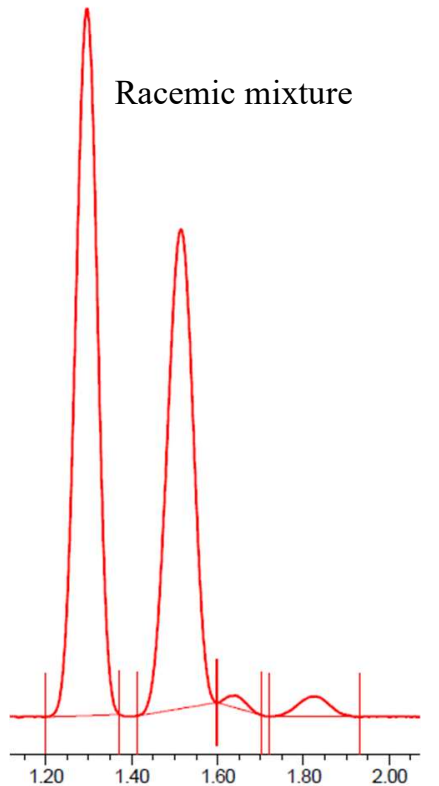

\begin{tabular}{|c|c|c|c|}
\hline $\begin{array}{c}\text { Retention Time } \\
(\text { mins })\end{array}$ & \% Area & $\begin{array}{c}\text { Target } \\
\text { Mass 1 } \\
(\mathrm{Da})\end{array}$ & $\begin{array}{c}\text { Base Peak } \\
(\mathrm{m} / \mathrm{z})\end{array}$ \\
\hline 1.296 & 53.52 & 364.40 & 321.27 \\
\hline 1.515 & 43.36 & 364.40 & 321.27 \\
\hline 1.645 & 0.83 & 364.40 & 365.31 \\
\hline 1.824 & 2.30 & 364.40 & 365.33 \\
\hline
\end{tabular}

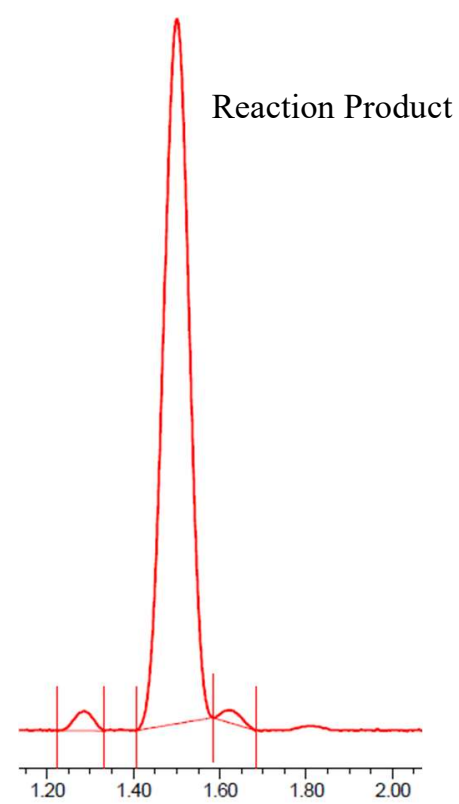

\begin{tabular}{|c|c|c|c|}
\hline $\begin{array}{c}\text { Retention Time } \\
(\text { mins })\end{array}$ & \% Area & $\begin{array}{c}\text { Target } \\
\text { Mass 1 } \\
(\mathrm{Da})\end{array}$ & $\begin{array}{c}\text { Base Peak } \\
(\mathrm{m} / \mathrm{z})\end{array}$ \\
\hline 1.286 & 2.02 & 364.40 & 321.26 \\
\hline 1.501 & 96.61 & 364.40 & 321.26 \\
\hline 1.630 & 1.37 & 364.40 & 365.33 \\
\hline
\end{tabular}




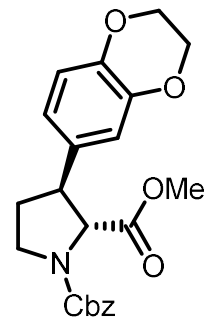

\section{1-benzyl 2-methyl (2R,3S)-3-(2,3-dihydrobenzo[b][1,4]dioxin-6-yl)pyrrolidine-1,2-}

dicarboxylate (19). The reaction was performed according to general procedure A with (1,4dihydrobenzo[d][1,2]dioxin-6-yl)boronic acid (135 mg, $0.75 \mathrm{mmol}, 1.5$ equiv). The crude residue was purified by silica gel chromatography (0-25\% EtOAc in hexanes) to afford the title compound as clear colorless oil (116 mg, 58\% yield, >20:1 dr, 94\% ee). ${ }^{1}$ H NMR (600 MHz, DMSO- $\left.d_{6}\right) \delta 7.41-7.36(\mathrm{~m}, 2 \mathrm{H}), 7.36-7.32(\mathrm{~m}, 1 \mathrm{H}), 7.31-7.27(\mathrm{~m}, 2 \mathrm{H}), 6.83(\mathrm{t}, J=2.4 \mathrm{~Hz}, 1 \mathrm{H})$, $6.81(\mathrm{~d}, J=6.2 \mathrm{~Hz}, 0.5 \mathrm{H}), 6.80(\mathrm{~d}, J=6.5 \mathrm{~Hz}, 0.5 \mathrm{H}), 6.75(\mathrm{~d}, J=1.9 \mathrm{~Hz}, 0.5 \mathrm{H}), 6.73(\mathrm{~d}, J=2.0 \mathrm{~Hz}, 0.5 \mathrm{H})$, $5.17-5.09(\mathrm{~m}, 1.5 \mathrm{H}), 4.99(\mathrm{~d}, J=12.7 \mathrm{~Hz}, 0.5 \mathrm{H}), 4.23(\mathrm{~d}, J=7.5 \mathrm{~Hz}, 0.5 \mathrm{H}), 4.21-4.18(\mathrm{~m}, 4.5 \mathrm{H}), 3.80$ $-3.65(\mathrm{~m}, 1 \mathrm{H}), 3.62(\mathrm{~s}, 1.5 \mathrm{H}), 3.49(\mathrm{~s}, 1.5 \mathrm{H}), 3.48-3.44(\mathrm{~m}, 1 \mathrm{H}), 3.36-3.29(\mathrm{~m}, 1 \mathrm{H}), 2.24-2.09(\mathrm{~m}$, 1H), $2.08-1.92(\mathrm{~m}, 1 \mathrm{H}) .{ }^{13} \mathbf{C}$ NMR (151 MHz, DMSO-d $\left.)\right) \delta 172.3,172.0,153.8,153.3,143.33,143.31$, $142.5,136.8,136.5,133.2,133.1,128.4,128.3,127.83,127.75,127.5,127.3,119.9,119.8,117.1,117.0$, 115.7, 115.6, 66.3, 66.2, 65.7, 65.3, 64.03, 63.98, 51.9, 51.8, 48.9, 47.8, 46.5, 46.0, 32.7, 32.0. HRESIMS $398.1610 \mathrm{~m} / \mathrm{z}$ (calc'd for $\mathrm{C}_{22} \mathrm{H}_{24} \mathrm{NO}_{6}{ }^{+}, \mathrm{m} / \mathrm{z} 398.1604 \mathrm{~m} / \mathrm{z} ; \Delta \mathrm{ppm} 1.5$ )

Analysis of Stereochemistry: The racemic material was prepared according to general procedure C (16.5:1 dr). Absolute stereochemistry was assigned by analogy (see section V).

SFC-MS: Lux-4 4.6x100, $3.5 \mathrm{ml} / \mathrm{min}, 20 \% \mathrm{MeOH} w / 0.1 \% \mathrm{NH}_{4} \mathrm{OH}, 2000 \mathrm{psi}$
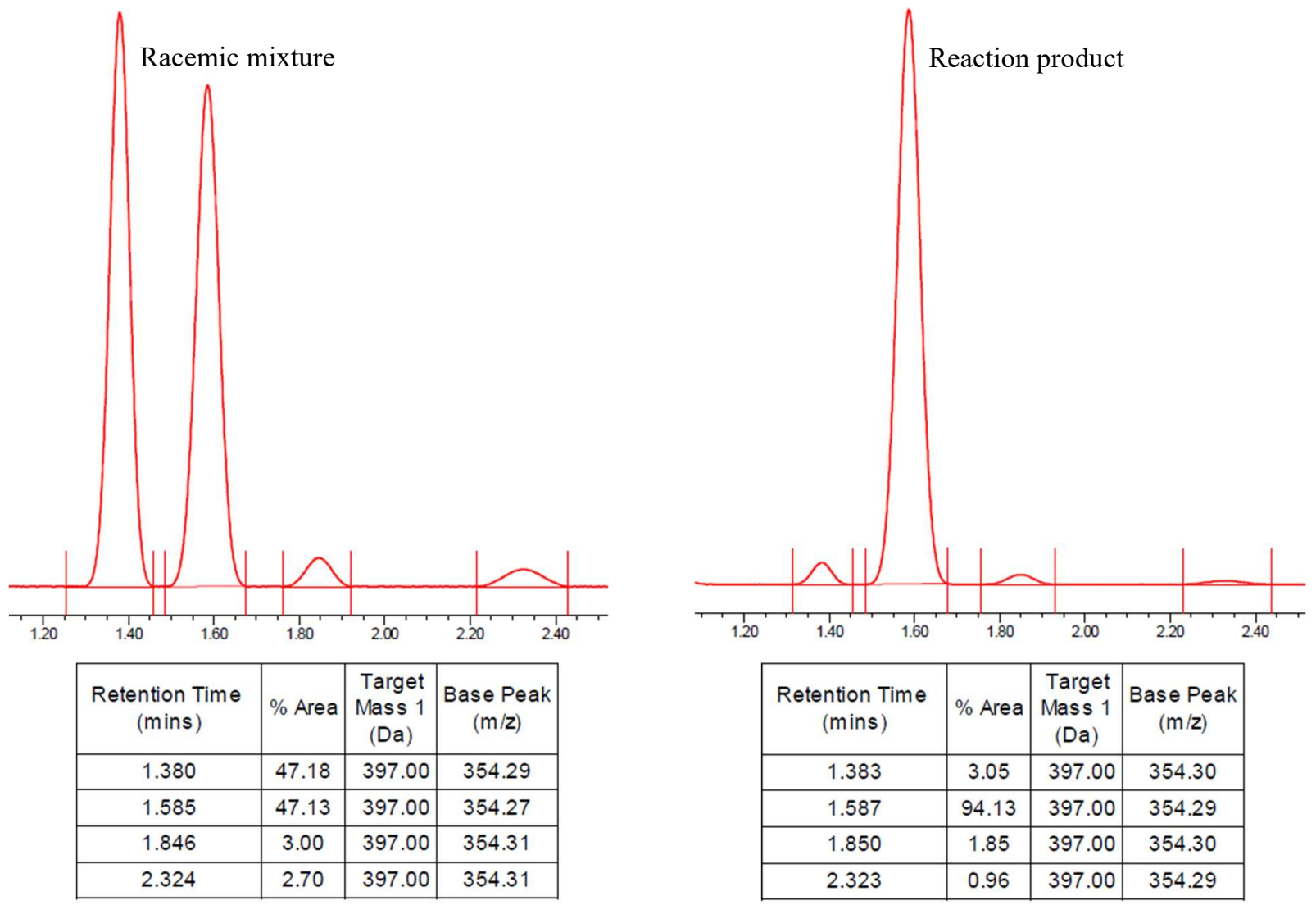


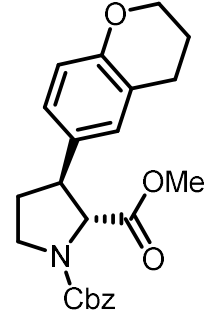

1-benzyl 2-methyl (2R,3S)-3-(chroman-6-yl)pyrrolidine-1,2-dicarboxylate (20). The reaction was performed according to general procedure A with chroman-6-ylboronic acid (133 mg, $0.75 \mathrm{mmol}, 1.5$ equiv). The crude residue was purified by silica gel chromatography $(0-40 \%$ EtOAc in hexanes) to afford the title compound as clear colorless oil (99.4 mg, 50\% yield, 19:1 dr, 95\% ee). ${ }^{1} \mathbf{H}$ NMR (600 MHz, DMSO- $d_{6}$ ) $\delta 7.42-7.38$ (m, 2H), $7.37-7.32(\mathrm{~m}, 1 \mathrm{H}), 7.32-7.27(\mathrm{~m}, 2 \mathrm{H}), 7.06-6.85(\mathrm{~m}, 2 \mathrm{H}), 6.69(\mathrm{t}, J=6.9 \mathrm{~Hz}, 1 \mathrm{H}), 5.27-$ $5.08(\mathrm{~m}, 1.5 \mathrm{H}), 5.00(\mathrm{~d}, J=12.7 \mathrm{~Hz}, 0.5 \mathrm{H}), 4.23(\mathrm{~d}, J=7.3 \mathrm{~Hz}, 0.5 \mathrm{H}), 4.21(\mathrm{~d}, J=7.3 \mathrm{~Hz}, 0.5 \mathrm{H}), 4.16-$ $4.04(\mathrm{~m}, 2 \mathrm{H}), 3.82-3.67(\mathrm{~m}, 1 \mathrm{H}), 3.62(\mathrm{~s}, 1.5 \mathrm{H}), 3.50(\mathrm{~s}, 1.5 \mathrm{H}), 3.55-3.44(\mathrm{~m}, 1 \mathrm{H}), 3.40-3.28(\mathrm{~m}, 1 \mathrm{H})$, $2.76-2.65(\mathrm{~m}, 2 \mathrm{H}), 2.29-2.12(\mathrm{~m}, 1 \mathrm{H}), 2.12-1.93(\mathrm{~m}, 1 \mathrm{H}), 1.93-1.80(\mathrm{~m}, 2 \mathrm{H}) .{ }^{13} \mathbf{C}$ NMR $(151 \mathrm{MHz}$, DMSO-d $\left.{ }_{6}\right) \delta 172.4,172.0,153.8,153.7,153.3,136.8,136.5,131.5,131.4,128.4,128.3,128.2,127.8$, 127.7, 127.5, 127.3, 125.74, 125.67, 122.3, 116.4, 116.3, 66.3, 66.2, 65.8, 65.4, 51.8, 51.7, 49.0, 47.9, 46.6, 46.0, 32.8, 32.1, 24.3, 21.8. HRESIMS $396.1820 \mathrm{~m} / \mathrm{z}$ (calc'd for $\mathrm{C}_{23} \mathrm{H}_{26} \mathrm{NO}_{5}{ }^{+}, \mathrm{m} / \mathrm{z} 396.1811 \mathrm{~m} / \mathrm{z} ; \Delta \mathrm{ppm}$ 2.3)

Analysis of Stereochemistry: The racemic material was prepared according to general procedure C (16.8:1 dr). Absolute stereochemistry was assigned by analogy (see section V).

SFC-MS: Lux-4 4.6x100, $3.5 \mathrm{ml} / \mathrm{min}, 20 \% \mathrm{MeOH} w / 0.1 \% \mathrm{NH}_{4} \mathrm{OH}, 2000 \mathrm{psi}$
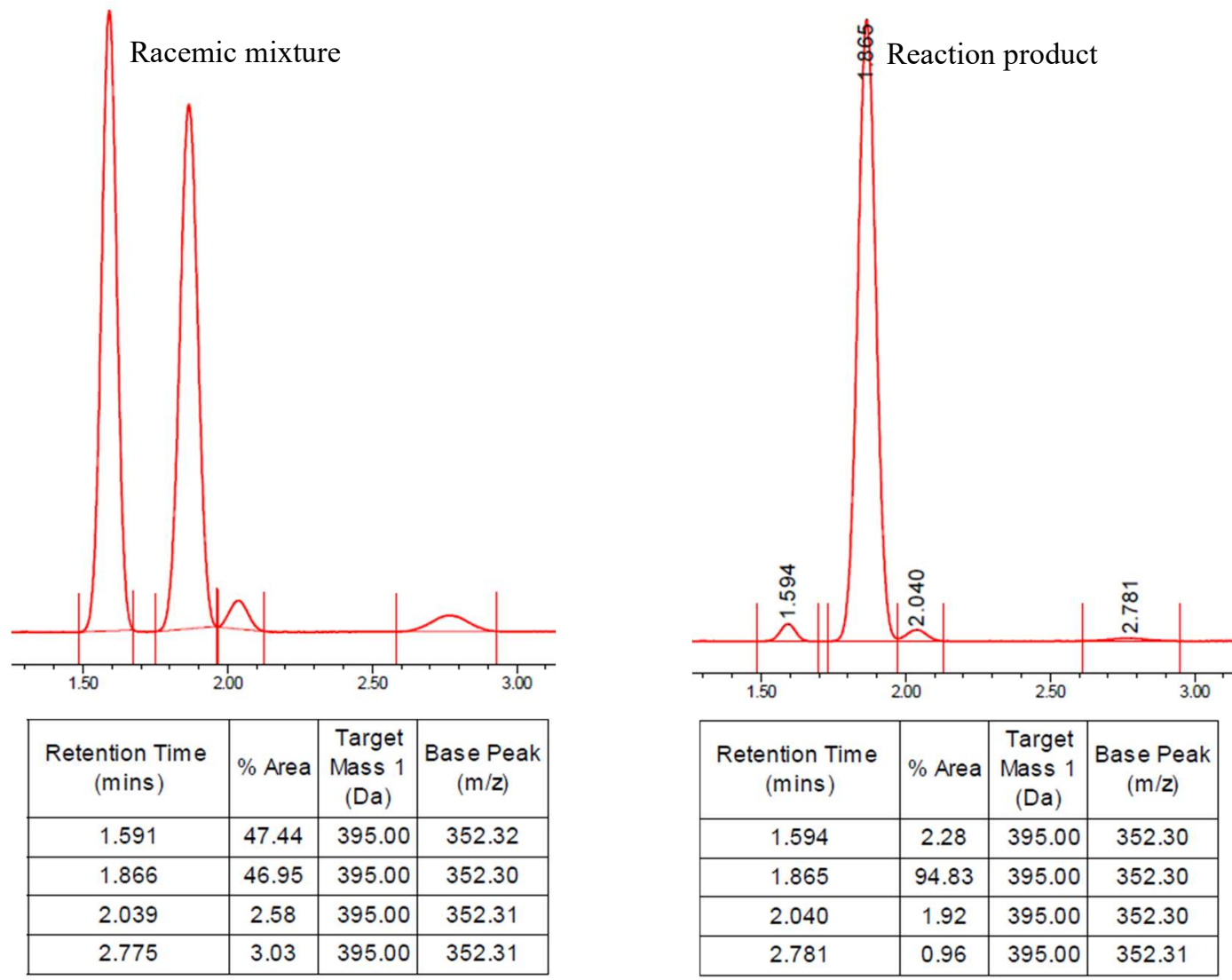


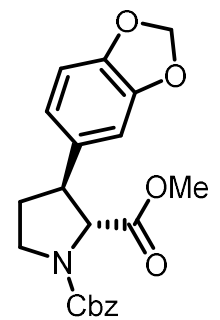

\section{1-benzyl 2-methyl (2R,3S)-3-(benzo[d][1,3]dioxol-5-yl)pyrrolidine-1,2-dicarboxylate}

(21). The reaction was performed according to general procedure A with benzo[d][1,3]dioxol-5-ylboronic acid (124 mg, $0.75 \mathrm{mmol}, 1.5$ equiv). The crude residue was purified by silica gel chromatography (0-40\% EtOAc in hexanes) to afford the title compound as clear colorless oil (92.5 mg, 48\% yield, 17:1 dr, 95\% ee). ${ }^{1} \mathbf{H}$ NMR (600 MHz, DMSO-d $\left.d_{6}\right) \quad 7.44-7.36(\mathrm{~m}, 2 \mathrm{H}), 7.33(\mathrm{~s}, 1 \mathrm{H}), 7.29(\mathrm{~d}, J=7.8 \mathrm{~Hz}, 2 \mathrm{H}), 6.99-6.93(\mathrm{~m}, 1 \mathrm{H}), 6.83(\mathrm{dd}, J=$ 8.0, $5.7 \mathrm{~Hz}, 1 \mathrm{H}), 6.74(\mathrm{~d}, J=7.6 \mathrm{~Hz}, 1 \mathrm{H}), 6.08-5.96(\mathrm{~m}, 2 \mathrm{H}), 5.18-5.08(\mathrm{~m}, 1.5 \mathrm{H}), 4.99(\mathrm{dd}, J=12.7$, $3.1 \mathrm{~Hz}, 0.5 \mathrm{H}), 4.24(\mathrm{dd}, J=7.8,3.8 \mathrm{~Hz}, 0.5 \mathrm{H}), 4.24-4.17(\mathrm{~m}, 0.5 \mathrm{H}), 3.79-3.67(\mathrm{~m}, 1 \mathrm{H}), 3.61(\mathrm{~s}, 1.5 \mathrm{H})$, $3.56-3.46(\mathrm{~m}, 1 \mathrm{H}), 3.49(\mathrm{~s}, 1.5 \mathrm{H}), 3.43-3.28(\mathrm{~m}, 1 \mathrm{H}), 2.30-2.11(\mathrm{~m}, 1 \mathrm{H}), 2.09-1.97(\mathrm{~m}, 1 \mathrm{H}) .{ }^{13} \mathbf{C}$ NMR $\left(151 \mathrm{MHz}, \mathrm{DMSO}-d_{6}\right) \delta 172.3,171.9,153.8,153.3,147.6,146.3,136.8,136.5,133.9,133.8,128.4$, $128.3,127.84,127.77,127.5,127.4,120.5,120.4,108.1,107.42,107.38,100.9,66.3,66.2,65.8,65.4$, 51.83, 51.78, 49.5, 48.4, 46.6, 46.1, 32.9, 32.2. HRESIMS $384.1452 \mathrm{~m} / \mathrm{z}$ (calc'd for $\mathrm{C}_{21} \mathrm{H}_{22} \mathrm{NO}_{4}{ }^{+}, \mathrm{m} / \mathrm{z}$ $384.1447 \mathrm{~m} / \mathrm{z} ; \Delta \mathrm{ppm} 1.3)$

Analysis of Stereochemistry: The racemic material was prepared according to general procedure C (14.7:1 dr). Absolute stereochemistry was assigned by analogy (see section V).

SFC-MS: Lux-4 4.6x100, $3.5 \mathrm{ml} / \mathrm{min}, 20 \% \mathrm{MeOH} w / 0.1 \% \mathrm{NH}_{4} \mathrm{OH}, 2000 \mathrm{psi}$
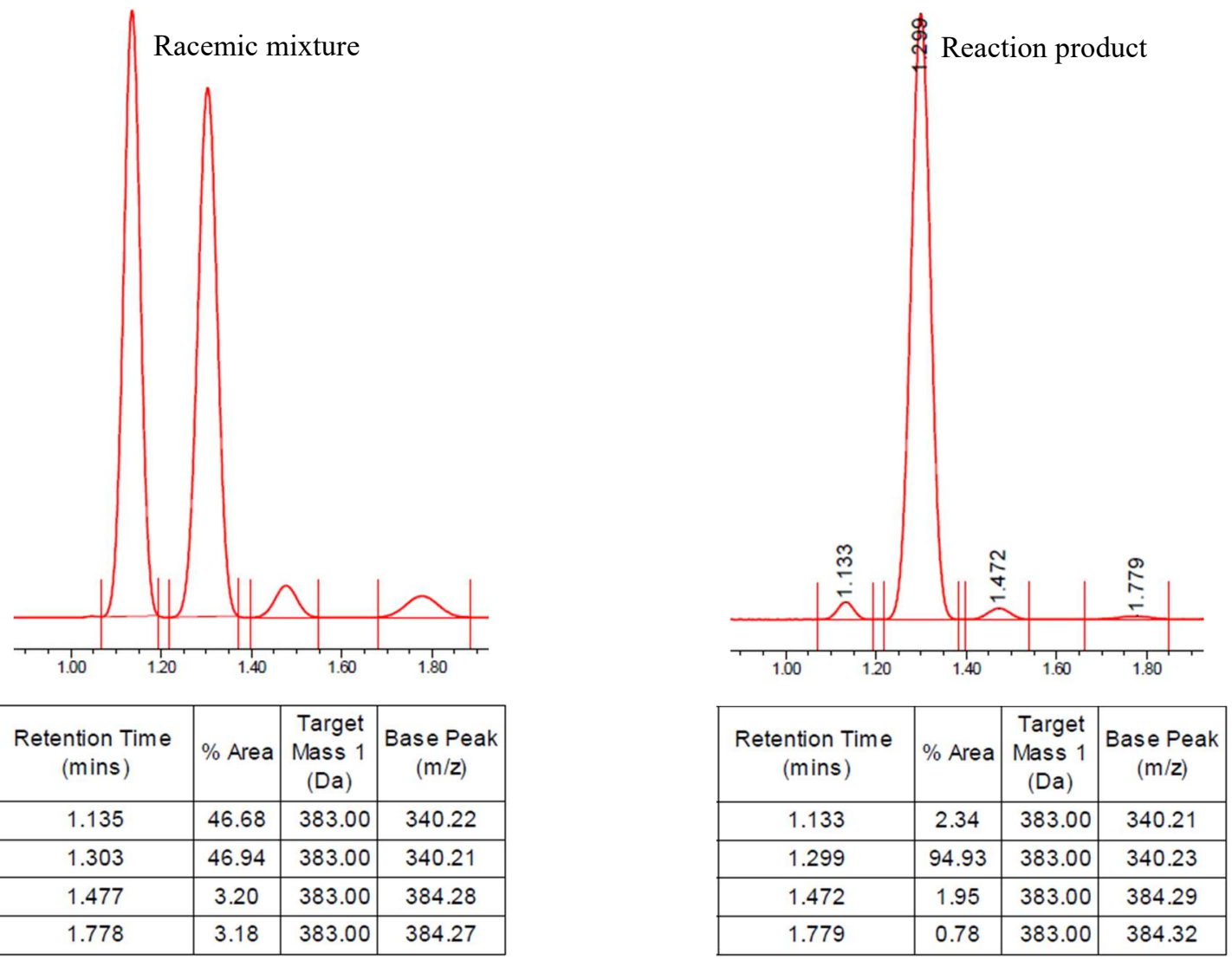

\begin{tabular}{|c|c|c|c|}
\hline $\begin{array}{c}\text { Retention Time } \\
(\text { mins })\end{array}$ & \% Area & $\begin{array}{c}\text { Target } \\
\text { Mass 1 } \\
(\mathrm{Da})\end{array}$ & $\begin{array}{c}\text { Base Peak } \\
(\mathrm{m} / \mathbf{z})\end{array}$ \\
\hline 1.135 & 46.68 & 383.00 & 340.22 \\
\hline 1.303 & 46.94 & 383.00 & 340.21 \\
\hline 1.477 & 3.20 & 383.00 & 384.28 \\
\hline 1.778 & 3.18 & 383.00 & 384.27 \\
\hline
\end{tabular}

\begin{tabular}{|c|c|c|c|}
\hline $\begin{array}{c}\text { Retention Time } \\
(\mathrm{mins})\end{array}$ & \% Area & $\begin{array}{c}\text { Target } \\
\text { Mass 1 } \\
(\mathrm{Da})\end{array}$ & $\begin{array}{c}\text { Base Peak } \\
(\mathrm{m} / \mathbf{z})\end{array}$ \\
\hline 1.133 & 2.34 & 383.00 & 340.21 \\
\hline 1.299 & 94.93 & 383.00 & 340.23 \\
\hline 1.472 & 1.95 & 383.00 & 384.29 \\
\hline 1.779 & 0.78 & 383.00 & 384.32 \\
\hline
\end{tabular}




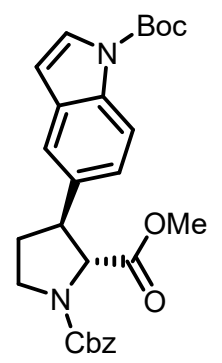

1-benzyl 2-methyl (2R,3S)-3-(1-(tert-butoxycarbonyl)-1H-indol-5-yl)pyrrolidine-1,2dicarboxylate (22). The reaction was performed according to general procedure A with (1(tert-butoxycarbonyl)-1H-indol-5-yl)boronic acid (196 mg, $0.75 \mathrm{mmol}, 1.5$ equiv). The crude residue was purified by silica gel chromatography $(0-40 \%$ EtOAc in hexanes) to afford the title compound as clear colorless oil (169 mg, 71\% yield, $>20: 1 \mathrm{dr}, 96 \% e e$ ). ${ }^{1} \mathbf{H}$ NMR $\left(600 \mathrm{MHz}\right.$, DMSO- $\left.d_{6}\right) \delta 8.05-7.97(\mathrm{~m}, 1 \mathrm{H}), 7.66(\mathrm{t}, J=3.3 \mathrm{~Hz}, 1 \mathrm{H}), 7.55-7.53(\mathrm{~m}$, 1H), $7.40-7.38(\mathrm{~m}, 2 \mathrm{H}), 7.37-7.32(\mathrm{~m}, 2 \mathrm{H}), 7.31-7.24(\mathrm{~m}, 2 \mathrm{H}), 6.66(\mathrm{t}, J=3.5 \mathrm{~Hz}, 1 \mathrm{H}), 5.18-5.09$ (m, 1.5H), 4.99 (d, $J=12.7 \mathrm{~Hz}, 0.5 \mathrm{H}), 4.32$ (d, $J=7.4 \mathrm{~Hz}, 0.5 \mathrm{H}), 4.29$ (d, $J=7.3 \mathrm{~Hz}, 0.5 \mathrm{H}), 3.74$ (ddd, $J$ $=10.9,7.8,3.4 \mathrm{~Hz}, 1 \mathrm{H}), 3.60(\mathrm{~s}, 1.5 \mathrm{H}), 3.58-3.48(\mathrm{~m}, 2 \mathrm{H}), 3.47(\mathrm{~s}, 1.5 \mathrm{H}), 2.25(\mathrm{dqd}, J=10.4,6.5,3.3$ $\mathrm{Hz}, 1 \mathrm{H}), 2.18-2.05$ (m, 1H), 1.61 (s, 9H). ${ }^{13} \mathbf{C}$ NMR (151 MHz, DMSO- $\left.d_{6}\right) \delta$ 172.3, 171.9, 153.8, 153.3, 149.0, 136.8, 136.5, 134.7, 134.6, 133.6, 130.44, 130.43, 128.4, 128.2, 127.8, 127.7, 127.5, 127.3, 126.4, $123.51,123.47,119.39,119.36,114.8,114.8,107.3,83.7,66.3,66.2,66.0,65.6,51.84,51.78,49.6,48.5$, 46.6, 46.1, 33.0, 32.3, 27.6. HRESIMS $479.2199 \mathrm{~m} / \mathrm{z}$ (calc'd for $\mathrm{C}_{27} \mathrm{H}_{31} \mathrm{~N}_{2} \mathrm{O}_{6}{ }^{+}, \mathrm{m} / \mathrm{z} 479.2182 \mathrm{~m} / \mathrm{z} ; \Delta \mathrm{ppm}$ 3.5)

Analysis of Stereochemistry: The racemic material was prepared according to general procedure C $(>20: 1$ dr). Absolute stereochemistry was assigned by analogy see section $\mathrm{V})$.

SFC-MS: Lux-4 4.6x100, 3.5 ml/min, 20\% MeOH w/ $0.1 \% \mathrm{NH}_{4} \mathrm{OH}, 2000 \mathrm{psi}$
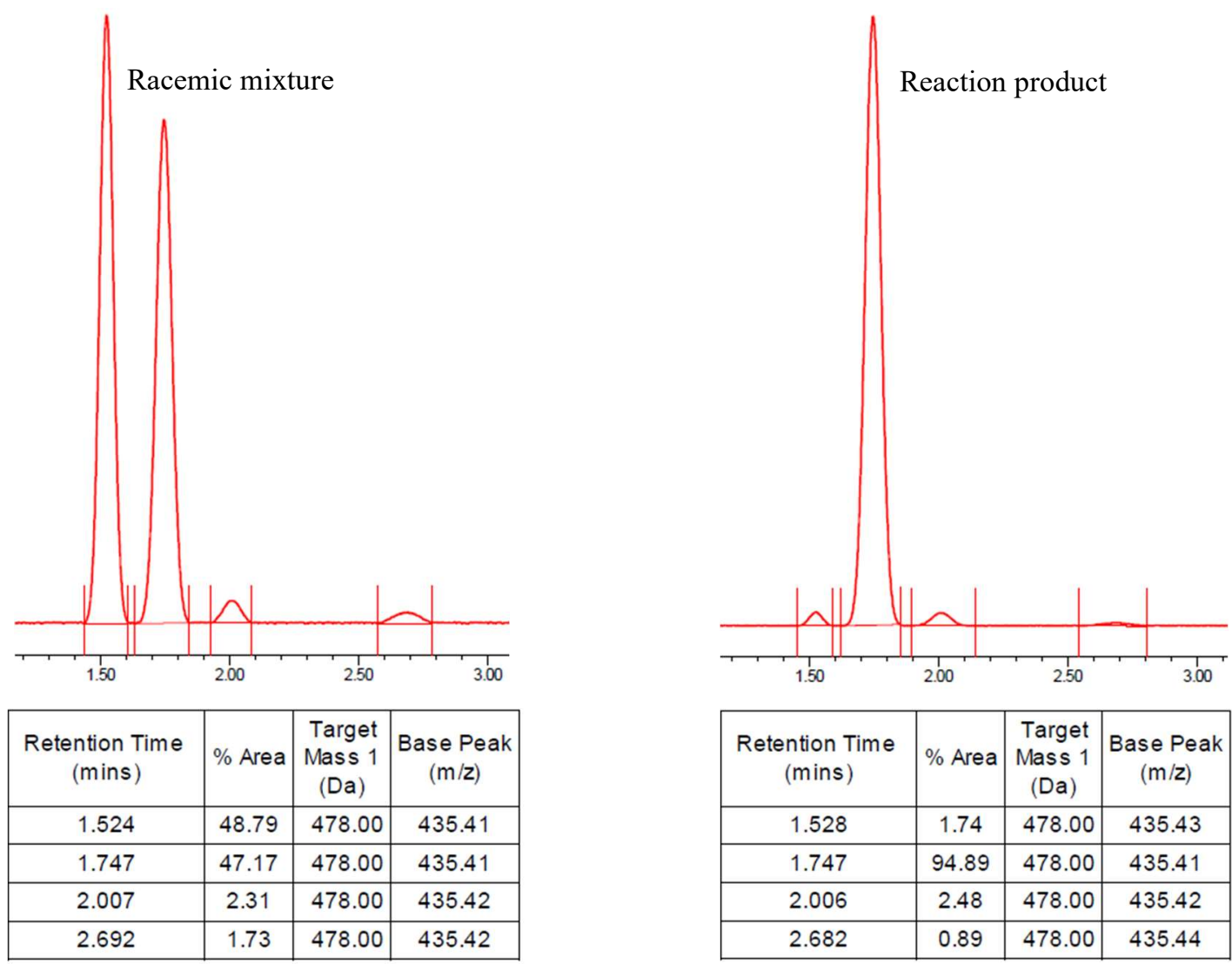


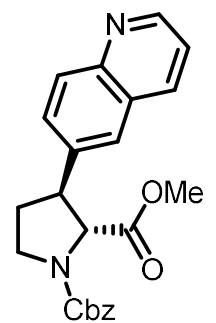

1-benzyl 2-methyl (2R,3S)-3-(quinolin-6-yl)pyrrolidine-1,2-dicarboxylate (23). The reaction was performed according to general procedure A with quinoline-6-ylboronic acid (130 mg, $0.75 \mathrm{mmol}, 1.5$ equiv). The crude residue was purified by silica gel chromatography (0-100\% EtOAc in hexanes) to afford the title compound as clear colorless oil (79.3 mg, 41\% yield, >20:1 dr, 64\% ee). ${ }^{1}$ H NMR (600 MHz, DMSO- $\left.d_{6}\right) \delta 8.92-8.82$ $(\mathrm{m}, 1 \mathrm{H}), 8.29(\mathrm{~s}, 0.5 \mathrm{H}), 8.28(\mathrm{~s}, 0.5 \mathrm{H}), 8.02(\mathrm{~d}, J=4.5 \mathrm{~Hz}, 0.5 \mathrm{H}), 8.01(\mathrm{~d}, J=4.5 \mathrm{~Hz}, 0.5 \mathrm{H}), 7.87(\mathrm{~d}, J=$ $2.2 \mathrm{~Hz}, 1 \mathrm{H}), 7.73(\mathrm{~s}, 0.5 \mathrm{H}), 7.71(\mathrm{~s}, 0.5 \mathrm{H}), 7.54-7.46(\mathrm{~m}, 1 \mathrm{H}), 7.41-7.35(\mathrm{~m}, 2 \mathrm{H}), 7.34-7.29(\mathrm{~m}, 1.5 \mathrm{H})$, $7.30-7.24(\mathrm{~m}, 1.5 \mathrm{H}), 5.24-5.10(\mathrm{~m}, 1.5 \mathrm{H}), 5.00(\mathrm{~d}, J=12.7 \mathrm{~Hz}, 0.5 \mathrm{H}), 4.43(\mathrm{~d}, J=7.4 \mathrm{~Hz}, 0.5 \mathrm{H}), 4.41$ $(\mathrm{d}, J=7.4 \mathrm{~Hz}, 0.5 \mathrm{H}), 3.84-3.72(\mathrm{~m}, 1 \mathrm{H}), 3.69-3.61(\mathrm{~m}, 1 \mathrm{H}), 3.58-3.55(\mathrm{~m}, 1 \mathrm{H}), 3.53(\mathrm{~s}, 1.5 \mathrm{H}), 3.48(\mathrm{~s}$, 1.5H), $2.37-2.23(\mathrm{~m}, 1 \mathrm{H}), 2.23-2.08(\mathrm{~m}, 1 \mathrm{H}) .{ }^{13} \mathbf{C}$ NMR $\left(151 \mathrm{MHz}, \mathrm{DMSO}-d_{6}\right) \delta 172.2,171.8,153.9$, 153.4, 150.4, 147.0, 138.4, 138.3, 136.7, 136.5, 135.8, 129.39, 129.37, 129.1, 129.0, 128.4, 128.3, 127.8, 127.8, 127.5, 127.4, 125.90, 125.85, 121.6, 66.4, 66.3, 65.6, 65.1, 52.0, 51.9, 49.5, 48.4, 46.7, 46.1, 32.7, 32.0. HRESIMS $413.1489 \mathrm{~m} / \mathrm{z}$ (calc'd for $\mathrm{C}_{23} \mathrm{H}_{22} \mathrm{NO}_{4} \mathrm{Na}^{+}, \mathrm{m} / \mathrm{z} 413.1477 \mathrm{~m} / \mathrm{z} ; \Delta \mathrm{ppm} 2.9$ )

Analysis of Stereochemistry: The racemic material was prepared according to general procedure C ( $>20: 1$ dr). Absolute stereochemistry was assigned by analogy (see section $\mathrm{V}$ ).

SFC-MS: Lux-4 4.6x100, $3.5 \mathrm{ml} / \mathrm{min}, 30 \% \mathrm{MeOH} w / 0.1 \% \mathrm{NH}_{4} \mathrm{OH}, 2000 \mathrm{psi}$
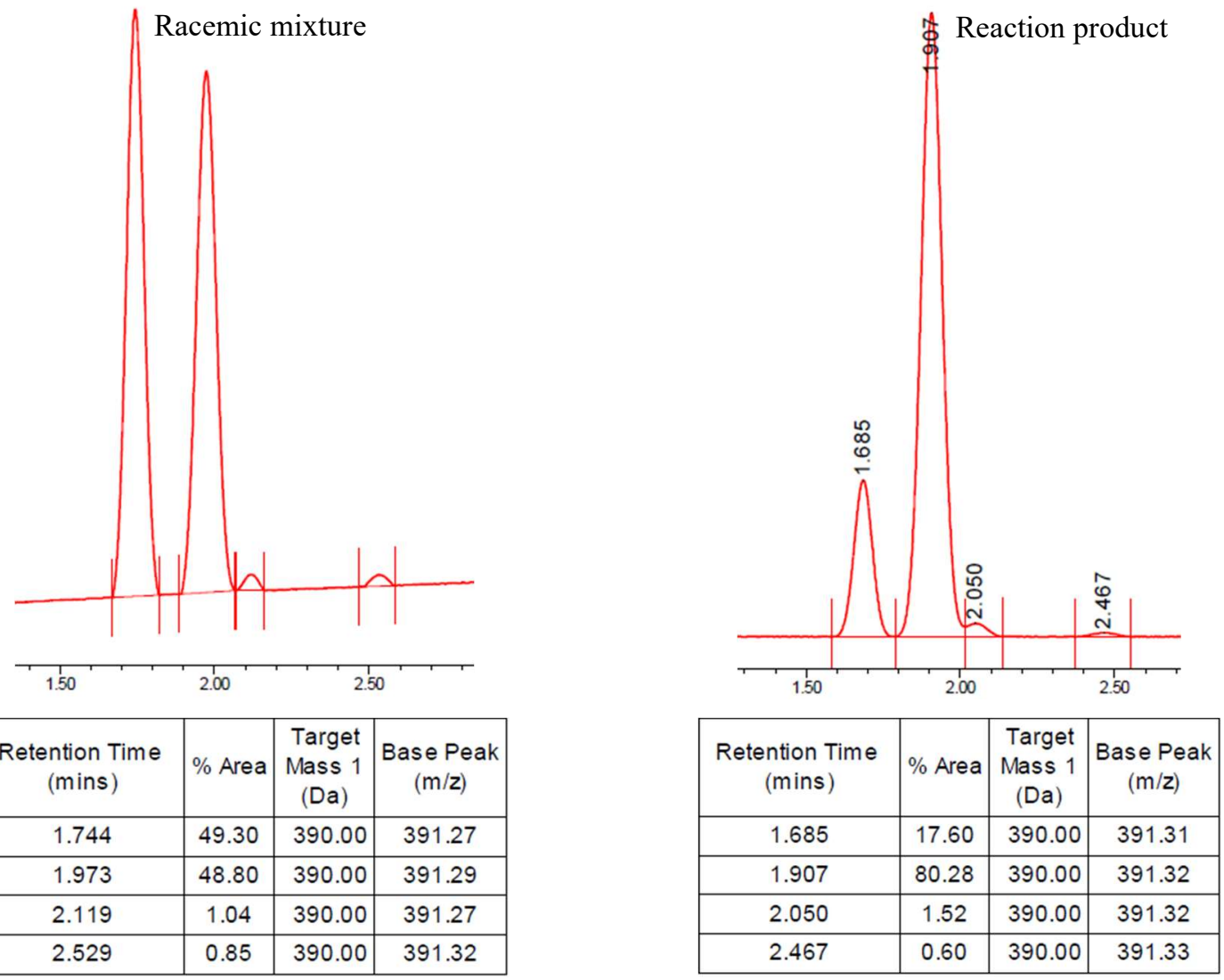


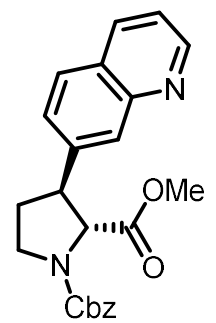

1-benzyl 2-methyl (2R,3S)-3-(quinolin-7-yl)pyrrolidine-1,2-dicarboxylate (24). The reaction was performed according to general procedure A with quinoline-6-ylboronic acid (130 mg, $0.75 \mathrm{mmol}, 1.5$ equiv). The crude residue was purified by silica gel chromatography (0-100\% EtOAc in hexanes) to afford the title compound as clear colorless oil (49.2 mg, 25\% yield, 6:1 dr, 69\% ee). ${ }^{1}$ H NMR (600 MHz, DMSO- $\left.d_{6}\right) \delta 8.90(\mathrm{td}, J=$ 4.0, $1.7 \mathrm{~Hz}, 1 \mathrm{H}), 8.34(\mathrm{t}, J=2.4 \mathrm{~Hz}, 0.5 \mathrm{H}), 8.33(\mathrm{~d}, J=2.5 \mathrm{~Hz}, 0.5 \mathrm{H}), 7.97(\mathrm{~d}, J=5.6 \mathrm{~Hz}, 0.5 \mathrm{H}), 7.96(\mathrm{~d}$, $J=5.5 \mathrm{~Hz}, 0.5 \mathrm{H}), 7.92(\mathrm{~s}, 1 \mathrm{H}), 7.62(\mathrm{t}, J=1.6 \mathrm{~Hz}, 0.5 \mathrm{H}), 7.60(\mathrm{t}, J=1.5 \mathrm{~Hz}, 0.5 \mathrm{H}), 7.54-7.48(\mathrm{~m}, 1 \mathrm{H})$, $7.42-7.37(\mathrm{~m}, 2 \mathrm{H}), 7.36-7.32(\mathrm{~m}, 1 \mathrm{H}), 7.32-7.22$ (m, 2H), 5.15-5.13 (m, 1.5H), $5.00(\mathrm{~d}, J=12.7 \mathrm{~Hz}$, $0.5 \mathrm{H}), 4.45(\mathrm{~d}, J=7.4 \mathrm{~Hz}, 0.5 \mathrm{H}), 4.41(\mathrm{~d}, J=7.2 \mathrm{~Hz}, 0.5 \mathrm{H}), 3.77$ (ddd, $J=10.8,7.8,3.5 \mathrm{~Hz}, 1 \mathrm{H}), 3.74-$ $3.66(\mathrm{~m}, 1 \mathrm{H}), 3.61(\mathrm{~s}, 1.5 \mathrm{H}), 3.60-3.51(\mathrm{~m}, 1 \mathrm{H}), 3.49(\mathrm{~s}, 1.5 \mathrm{H}), 2.38-2.29(\mathrm{~m}, 1 \mathrm{H}), 2.27-2.16(\mathrm{~m}, 1 \mathrm{H})$. ${ }^{13}$ C NMR (151 MHz, DMSO- $\left.d_{6}\right) \delta 172.1,171.8,153.9,153.4,150.8,147.7,141.8,141.6,136.7,136.5$, $135.7,128.6,128.5,128.4,128.3,127.9,127.8,127.5,127.3,127.0,126.9,126.8,126.1,121.4,66.3,66.2$, 65.4, 65.0, 52.0, 51.9, 49.5, 48.4, 46.7, 46.1, 32.6, 31.9. HRESIMS $391.1674 \mathrm{~m} / \mathrm{z}$ (calc'd for $\mathrm{C}_{23} \mathrm{H}_{23} \mathrm{~N}_{2} \mathrm{O}_{4}{ }^{+}$, $\mathrm{m} / \mathrm{z} 391.1658 \mathrm{~m} / \mathrm{z} ; \Delta \mathrm{ppm} 4.1)$

Analysis of Stereochemistry: The racemic material was prepared according to general procedure C (12.4:1 dr). Absolute stereochemistry was assigned by analogy (see section $\mathrm{V}$ ).

SFC-MS: (R,R)-Whelk-01 4.6x100, $3.5 \mathrm{ml} / \mathrm{min}, 25 \% \mathrm{MeOH} w / 0.1 \% \mathrm{NH}_{4} \mathrm{OH}, 2000 \mathrm{psi}$

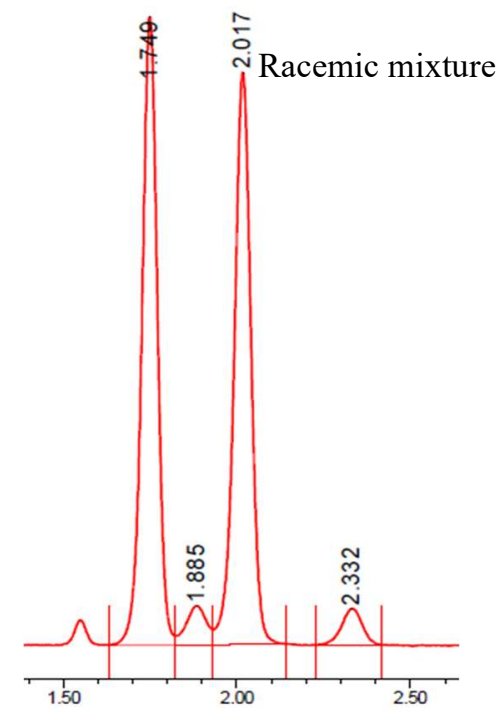

\begin{tabular}{|c|c|c|c|}
\hline $\begin{array}{c}\text { Retention Time } \\
(\mathrm{mins})\end{array}$ & \% Area & $\begin{array}{c}\text { Target } \\
\text { Mass 1 } \\
(\mathrm{Da})\end{array}$ & $\begin{array}{c}\text { Base Peak } \\
(\mathrm{m} / \mathrm{z})\end{array}$ \\
\hline 1.749 & 46.31 & 390.00 & 391.31 \\
\hline 1.885 & 3.80 & 390.00 & 391.32 \\
\hline 2.017 & 46.25 & 390.00 & 391.33 \\
\hline 2.332 & 3.65 & 390.00 & 391.33 \\
\hline
\end{tabular}

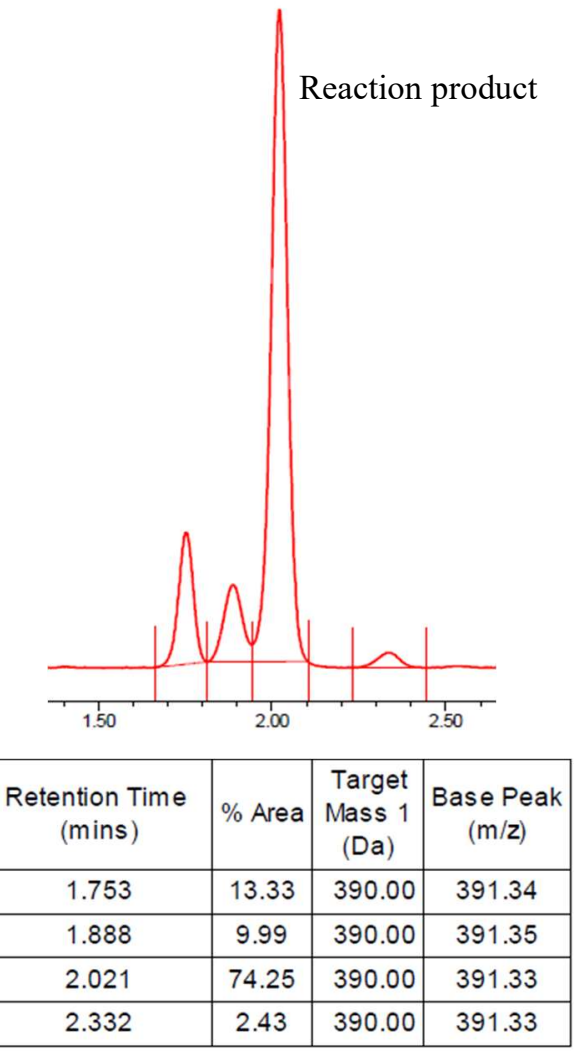


$\sum_{\mathrm{Cbz}}^{\mathrm{OMe}}$

1-benzyl 2-methyl (2R,3S)-3-(pyridin-4-yl)pyrrolidine-1,2-dicarboxylate (25). The reaction was performed according to general procedure D with pyridin-4-ylboronic acid (184 mg, $1.5 \mathrm{mmol}, 3.0$ equiv). The crude residue was purified by silica gel chromatography (0-100\% EtOAc in hexanes) to afford the title compound as clear colorless oil (57.2 $\mathrm{mg}$, $34 \%$ yield, $2.5: 1 \mathrm{dr}$, $4 \%$ ee for the trans isomer, $81 \%$ ee for the cis enantiomer).

trans isomer: ${ }^{1} \mathbf{H}$ NMR $\left(600 \mathrm{MHz}, \mathrm{DMSO}-d_{6}\right) \delta 8.55-8.50(\mathrm{~m}, 2 \mathrm{H}), 7.42-7.39(\mathrm{~m}, 2 \mathrm{H}), 7.38-7.33(\mathrm{~m}$, $3 \mathrm{H}), 7.32-7.24(\mathrm{~m}, 2 \mathrm{H}), 5.17-5.12(\mathrm{~m}, 1.5 \mathrm{H}), 5.00(\mathrm{~d}, J=12.6 \mathrm{~Hz}, 0.5 \mathrm{H}), 4.37(\mathrm{~d}, J=7.2 \mathrm{~Hz}, 0.5 \mathrm{H})$, $4.34(\mathrm{~d}, J=7.1 \mathrm{~Hz}, 0.5 \mathrm{H}), 3.72(\mathrm{ddd}, J=11.0,7.8,3.6 \mathrm{~Hz}, 1 \mathrm{H}), 3.63(\mathrm{~s}, 1.5 \mathrm{H}), 3.51(\mathrm{~s}, 1.5 \mathrm{H}), 3.59-3.43$ (m, 2H), $2.33-2.20(\mathrm{~m}, 1 \mathrm{H}), 2.11-2.01(\mathrm{~m}, 1 \mathrm{H}) .{ }^{13} \mathbf{C}$ NMR (151 MHz, DMSO-d $) \delta 171.9,171.5,153.8$, $153.3,149.9,149.5,128.4,128.3,127.51,127.49,127.4,122.6,122.5,66.34,66.27,64.8,64.3,52.02$, 51.97, 48.4, 47.3, 32.1, 31.4. HRESIMS $341.1495 \mathrm{~m} / \mathrm{z}$ (calc'd for $\mathrm{C}_{19} \mathrm{H}_{21} \mathrm{~N}_{2} \mathrm{O}_{4}{ }^{+}, \mathrm{m} / \mathrm{z} 341.1501 \mathrm{~m} / \mathrm{z} ; \Delta \mathrm{ppm}$ 01.8).

Analysis of Stereochemistry: The racemic material was prepared according to general procedure D with rac-Ph-BPE-Rh. Absolute stereochemistry was assigned by analogy (see section $\mathrm{V}$ ).

SFC-MS: AD-3 4.6x100, $3.5 \mathrm{ml} / \mathrm{min}, 25 \% \mathrm{MeOH} w / 0.1 \% \mathrm{NH}_{4} \mathrm{OH}, 2000 \mathrm{psi}$
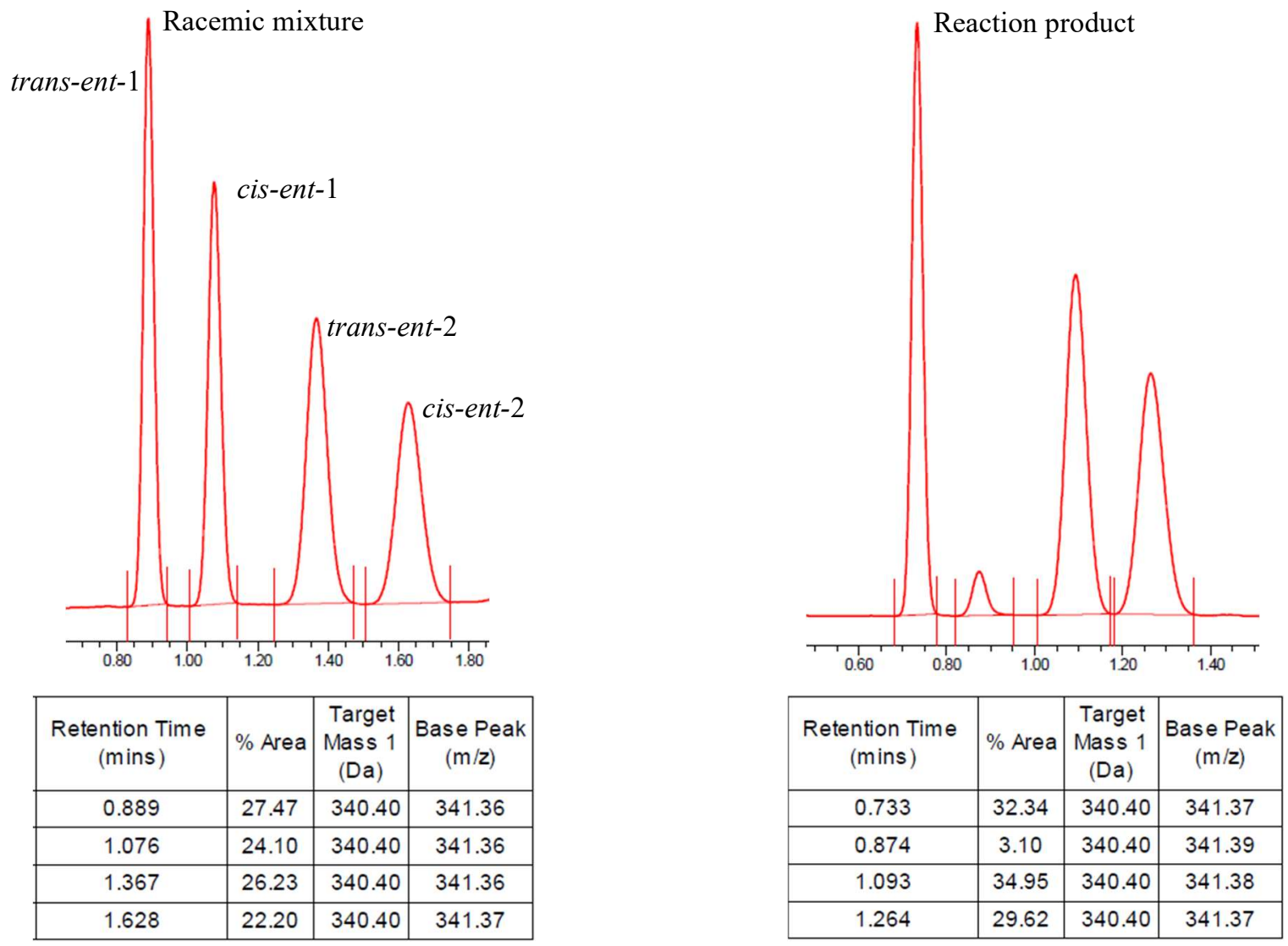


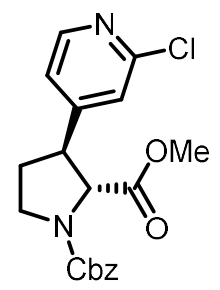

1-benzyl 2-methyl (2R,3S)-3-(2-chloropyridin-4-yl)pyrrolidine-1,2-dicarboxylate

(26).

The reaction was performed according to general procedure D with (2-chloropyridin-4yl)boronic acid (236 mg, $1.5 \mathrm{mmol}, 3.0$ equiv). The crude residue was purified by silica gel chromatography (0-50\% EtOAc in hexanes) to afford the title compound as viscous clear colorless oil (75.9 mg, 41\% yield, $1.7: 1 \mathrm{dr}, 2 \%$ ee for the trans isomer, $81 \%$ ee for the cis isomer).

trans isomer: ${ }^{1} \mathbf{H}$ NMR $\left(600 \mathrm{MHz}, \mathrm{DMSO}-d_{6}\right) \delta 8.41-8.31(\mathrm{~m}, 1 \mathrm{H}), 7.55(\mathrm{~s}, 1 \mathrm{H}), 7.40(\mathrm{dd}, J=5.3,1.6$ $\mathrm{Hz}, 1 \mathrm{H}), 7.39(\mathrm{~d}, J=3.4 \mathrm{~Hz}, 2 \mathrm{H}), 7.34(\mathrm{~m}, 1 \mathrm{H}), 7.31-7.28(\mathrm{~m}, 2 \mathrm{H}), 5.15-5.07(\mathrm{~m}, 1.5 \mathrm{H}), 4.99(\mathrm{~d}, J=$ $12.6 \mathrm{~Hz}, 0.5 \mathrm{H}), 4.41(\mathrm{~d}, J=7.5 \mathrm{~Hz}, 0.5 \mathrm{H}), 4.38(\mathrm{~d}, J=7.4 \mathrm{~Hz}, 0.5 \mathrm{H}), 3.73$ (ddd, $J=10.9,7.8,3.3 \mathrm{~Hz}, 1 \mathrm{H})$, $3.63(\mathrm{~s}, 1.5 \mathrm{H}), 3.59-3.53(\mathrm{~m}, 1 \mathrm{H}), 3.52(\mathrm{~s}, 1.5 \mathrm{H}) 3.51-3.35(\mathrm{~m}, 1 \mathrm{H}), 2.27(\mathrm{ttd}, J=13.1,6.6,3.1 \mathrm{~Hz}, 1 \mathrm{H})$, 2.09 (ddd, $J=20.7,11.9,8.6 \mathrm{~Hz}, 1 \mathrm{H}) .{ }^{13} \mathbf{C}$ NMR (151 MHz, DMSO-d $\left.)\right) \delta 171.7,171.3,153.8,153.31$, 153.26, 153.2, 150.7, 150.1, 149.7, 128.4, 128.3, 127.5, 127.4, 123.1, 122.1, 122.0, 66.4, 66.3, 64.6, 64.1, 52.04, 52.00, 48.1, 47.0, 46.5, 46.0, 32.2, 31.5. HRESIMS $375.1130 \mathrm{~m} / \mathrm{z}$ (calc'd for $\mathrm{C}_{19} \mathrm{H}_{20} \mathrm{~N}_{2} \mathrm{O}_{4} \mathrm{Cl}^{+}, \mathrm{m} / \mathrm{z}$ $375.1112 \mathrm{~m} / \mathrm{z} ; \Delta \mathrm{ppm} 4.8)$.

Analysis of Stereochemistry: The racemic material was prepared according to general procedure D with rac-Ph-BPE-Rh. Absolute stereochemistry was assigned by analogy (see section V).

SFC-MS: IF 4.6x100, $3.5 \mathrm{ml} / \mathrm{min}, 15 \% \mathrm{MeOH} w / 0.1 \% \mathrm{NH}_{4} \mathrm{OH}, 2000 \mathrm{psi}$

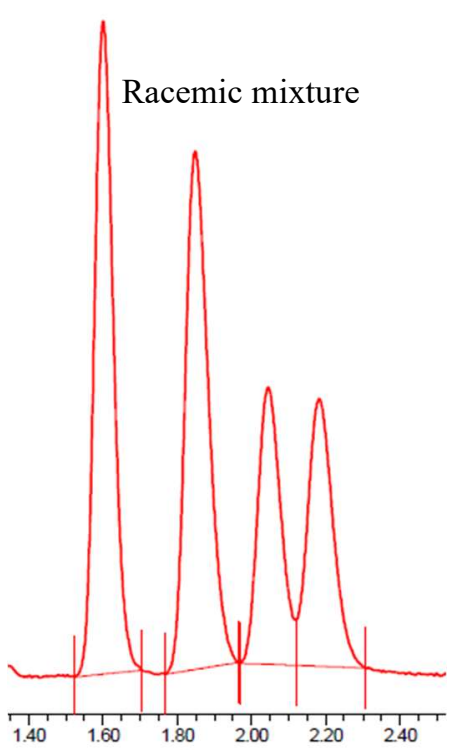

\begin{tabular}{|c|c|c|c|}
\hline $\begin{array}{c}\text { Retention Time } \\
(\mathrm{mins})\end{array}$ & \% Area & $\begin{array}{c}\text { Target } \\
\text { Mass 1 } \\
(\mathrm{Da})\end{array}$ & $\begin{array}{c}\text { Base Peak } \\
(\mathrm{m} / \mathbf{z})\end{array}$ \\
\hline 1.600 & 32.79 & 374.00 & 375.40 \\
\hline 1.848 & 31.82 & 374.00 & 375.39 \\
\hline 2.045 & 17.10 & 374.00 & 375.37 \\
\hline 2.183 & 18.28 & 374.00 & 375.41 \\
\hline
\end{tabular}

Reaction product

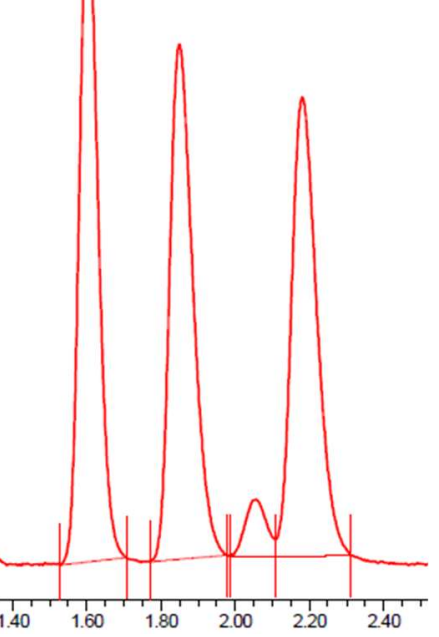

\begin{tabular}{|c|c|c|c|}
\hline $\begin{array}{c}\text { Retention Time } \\
(\mathrm{mins})\end{array}$ & \% Area & $\begin{array}{c}\text { Target } \\
\text { Mass 1 } \\
(\mathrm{Da})\end{array}$ & $\begin{array}{c}\text { Base Peak } \\
(\mathrm{m} / \mathbf{z})\end{array}$ \\
\hline 1.602 & 33.36 & 374.00 & 375.40 \\
\hline 1.849 & 32.07 & 374.00 & 375.41 \\
\hline 2.056 & 3.25 & 374.00 & 375.39 \\
\hline 2.181 & 31.32 & 374.00 & 375.40 \\
\hline
\end{tabular}




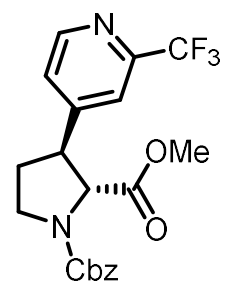

\section{1-benzyl 2-methyl (2R,3S)-3-(2-(trifluoromethyl)pyridin-4-yl)pyrrolidine-1,2-}

dicarboxylate (27). The reaction was performed according to general procedure D with (2(trifluoromethyl)pyridin-4-yl)boronic acid (286 mg, $1.5 \mathrm{mmol}, 3.0$ equiv). The crude residue was purified by silica gel chromatography (0-50\% EtOAc in hexanes) to afford the title compound as viscous clear colorless oil $(80.0 \mathrm{mg}, 39 \%$ yield, $2.5: 1 \mathrm{dr}, 53 \%$ ee for the trans isomer, $72 \%$ ee for the cis enantiomer).

trans isomer: ${ }^{1} \mathbf{H}$ NMR $\left(600 \mathrm{MHz}, \mathrm{DMSO}-d_{6}\right) \delta 8.81-8.67(\mathrm{~m}, 1 \mathrm{H}), 7.95(\mathrm{~d}, J=4.2 \mathrm{~Hz}, 1 \mathrm{H}), 7.71(\mathrm{dd}, J$ $=5.1,1.6 \mathrm{~Hz}, 1 \mathrm{H}), 7.43-7.36(\mathrm{~m}, 2 \mathrm{H}), 7.36-7.31(\mathrm{~m}, 1 \mathrm{H}), 7.31-7.26(\mathrm{~m}, 2 \mathrm{H}), 5.16-5.14(\mathrm{~m}, 1.5 \mathrm{H})$, $4.99(\mathrm{~d}, J=12.7 \mathrm{~Hz}, 0.5 \mathrm{H}), 4.47(\mathrm{~d}, J=7.7 \mathrm{~Hz}, 0.5 \mathrm{H}), 4.44(\mathrm{~d}, J=7.5 \mathrm{~Hz}, 0.5 \mathrm{H}), 3.78-3.73(\mathrm{~m}, 1 \mathrm{H}), 3.72$ $-3.63(\mathrm{~m}, 1 \mathrm{H}), 3.63(\mathrm{~s}, 1.5 \mathrm{H}), 3.58-3.53(\mathrm{~m}, 1 \mathrm{H}), 3.52(\mathrm{~s}, 1.5 \mathrm{H}), 2.36-2.27(\mathrm{~m}, 1 \mathrm{H}), 2.19-2.09(\mathrm{~m}$, 1H). ${ }^{13}$ C NMR (151 MHz, DMSO- $\left.d_{6}\right) \delta 171.7,171.3,153.8,153.3,151.9,151.8,150.5,147.3-146.7$ (q, $J$ $=33.1,1 \mathrm{C}), 136.7,136.4,128.4,128.3,127.9,127.8,127.5,127.4,127.3,66.4,66.3,64.7,64.2,52.01$, 51.97, 48.4, 47.3, 46.6, 46.1, 32.3, 31.6. ${ }^{19}$ F NMR (564 MHz, DMSO- $d_{6}$ ) 66.39, 66.40. HRESIMS $408.1358 \mathrm{~m} / \mathrm{z}$ (calc'd for $\mathrm{C}_{20} \mathrm{H}_{20} \mathrm{~N}_{2} \mathrm{O}_{4} \mathrm{~F}_{3}{ }^{+}, \mathrm{m} / \mathrm{z} 408.1375 \mathrm{~m} / \mathrm{z} ; \Delta \mathrm{ppm} 4.8$ )

Analysis of Stereochemistry: The racemic material was prepared according to general procedure D with rac-Ph-BPE-Rh. Absolute stereochemistry was assigned by analogy (see section V).

SFC-MS: AD-3 4.6x100, $3.5 \mathrm{ml} / \mathrm{min}, 20 \% \mathrm{MeOH} w / 0.1 \% \mathrm{NH}_{4} \mathrm{OH}, 2000 \mathrm{psi}$
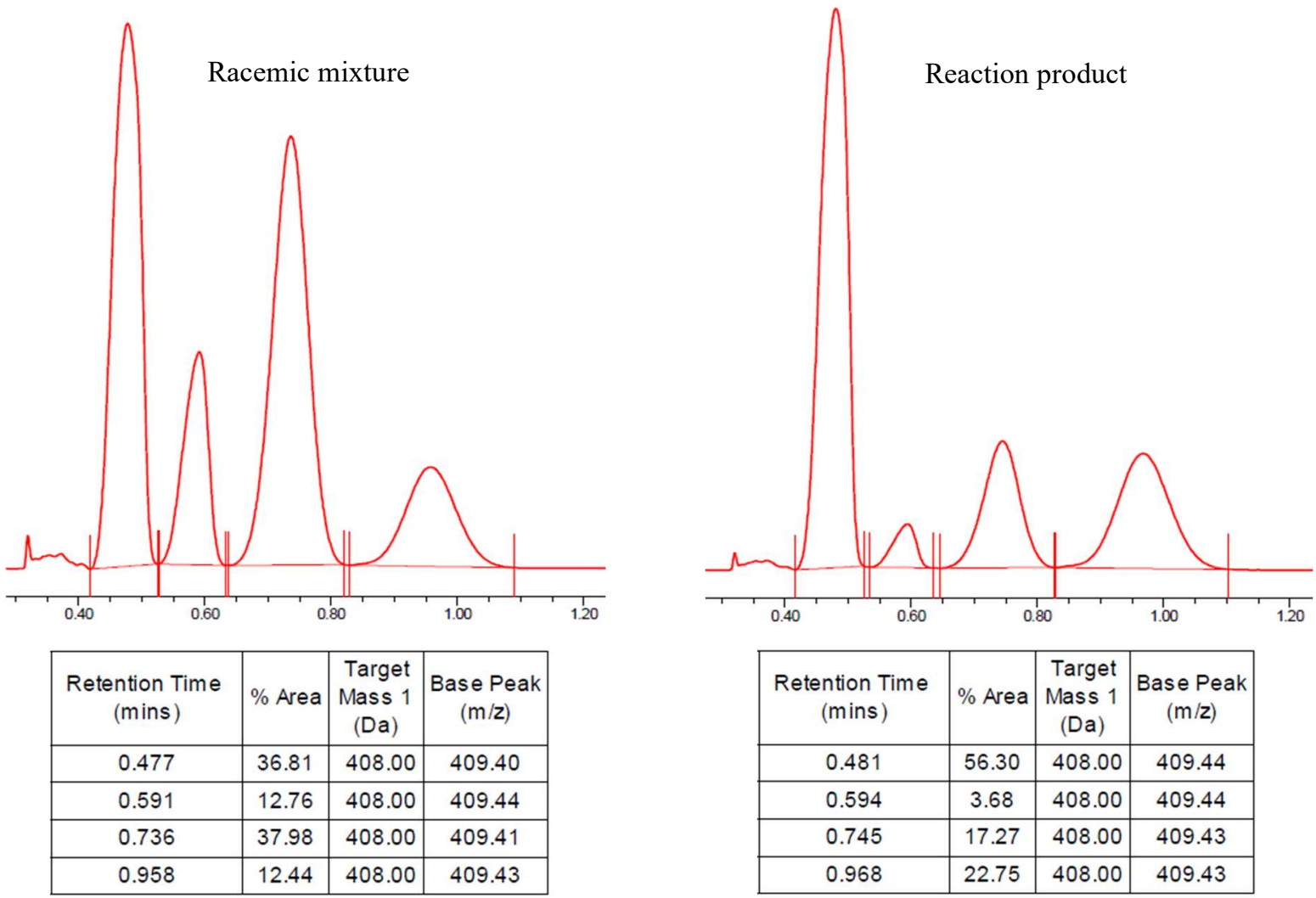

\begin{tabular}{|c|c|c|c|}
\hline $\begin{array}{c}\text { Retention Time } \\
(\mathrm{mins})\end{array}$ & \% Area & $\begin{array}{c}\text { Target } \\
\text { Mass 1 } \\
(\mathrm{Da})\end{array}$ & $\begin{array}{c}\text { Base Peak } \\
(\mathrm{m} / \mathrm{z})\end{array}$ \\
\hline 0.477 & 36.81 & 408.00 & 409.40 \\
\hline 0.591 & 12.76 & 408.00 & 409.44 \\
\hline 0.736 & 37.98 & 408.00 & 409.41 \\
\hline 0.958 & 12.44 & 408.00 & 409.43 \\
\hline
\end{tabular}




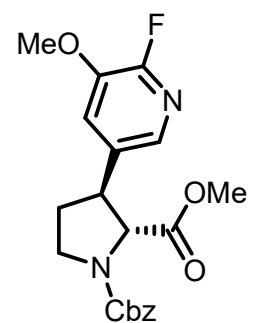

\section{1-benzyl 2-methyl (2R,3S)-3-(6-fluoro-5-methoxypyridin-3-yl)pyrrolidine-1,2-}

dicarboxylate (28). The reaction was performed according to general procedure $\mathrm{D}$ with (6-fluoro-5-methoxypyridin-3-yl)boronic acid (256 mg, $1.5 \mathrm{mmol}, 3.0$ equiv). The crude residue was purified by silica gel chromatography $(0-50 \%$ EtOAc in hexanes) to afford the title compound as viscous clear colorless oil (43.4 mg, 22\% yield, 9:1 dr, 90\% ee). ${ }^{1} \mathbf{H}$ NMR $\left(600 \mathrm{MHz}, \mathrm{DMSO}-d_{6}\right) \delta 7.91(\mathrm{~d}, J=1.5 \mathrm{~Hz}, 1 \mathrm{H}), 7.82(\mathrm{dd}, J=5.1,2.0 \mathrm{~Hz}, 0.5 \mathrm{H}), 7.80(\mathrm{dd}, J=5.1$, $2.0 \mathrm{~Hz}, 0.5 \mathrm{H}), 7.39(\mathrm{~d}, J=4.4 \mathrm{~Hz}, 2 \mathrm{H}), 7.36-7.31(\mathrm{~m}, 1.5 \mathrm{H}), 7.31-7.26(\mathrm{~m}, 1.5 \mathrm{H}), 5.19-5.07(\mathrm{~m}, 1.5 \mathrm{H})$, $4.97(\mathrm{~d}, J=12.7 \mathrm{~Hz}, 0.5 \mathrm{H}), 4.28(\mathrm{~d}, J=8.3 \mathrm{~Hz}, 0.5 \mathrm{H}), 4.24(\mathrm{~d}, J=8.2 \mathrm{~Hz}, 0.5 \mathrm{H}), 3.93(\mathrm{~s}, 1.5 \mathrm{H}), 3.92(\mathrm{~s}$, 1.5H), 3.74 (ddt, $J=10.2,8.0,2.3 \mathrm{~Hz}, 1 \mathrm{H}), 3.61(\mathrm{~s}, 1.5 \mathrm{H}), 3.48(\mathrm{~s}, 1.5 \mathrm{H}), 3.48-3.39(\mathrm{~m}, 1 \mathrm{H}), 2.20(\mathrm{ttd}, J$ $=12.5,6.5,2.3 \mathrm{~Hz}, 1 \mathrm{H}), 2.16-1.98(\mathrm{~m}, 1 \mathrm{H}) .{ }^{13} \mathbf{C} \mathbf{N M R}\left(151 \mathrm{MHz}, \mathrm{DMSO}-d_{6}\right) \delta 171.9,171.5,153.8,153.3$, $151.9,151.8,147.5,145.8,140.28,140.25,140.22$, 140.18, 136.7, 136.4, 129.9, 129.7, 128.4, 128.3, 127.9, 127.8, 127.6, 127.5, 127.4, 127.2, 122.7, 122.6, 66.3, 66.2, 65.3, 64.9, 53.5, 52.0, 51.9, 46.7, 46.2, 46.1, 45.1, 32.7, 32.0. ${ }^{19}$ F NMR (564 MHz, DMSO- $\left.d_{6}\right)-139.80--139.85(\mathrm{~m})$. HRESIMS $389.1527 \mathrm{~m} / \mathrm{z}$ (calc'd for $\mathrm{C}_{20} \mathrm{H}_{22} \mathrm{~N}_{2} \mathrm{O}_{5} \mathrm{~F}^{+}, \mathrm{m} / \mathrm{z} 389.1513 \mathrm{~m} / \mathrm{z} ; \Delta \mathrm{ppm} 3.6$ )

Analysis of Stereochemistry: The racemic material was prepared according to general procedure D with rac-Ph-BPE-Rh. Absolute stereochemistry was assigned by analogy (see section V).

SFC-MS: Lux-2 4.6x100, $3.5 \mathrm{ml} / \mathrm{min}, 20 \% \mathrm{MeOH} w / 0.1 \% \mathrm{NH}_{4} \mathrm{OH}, 2000 \mathrm{psi}$
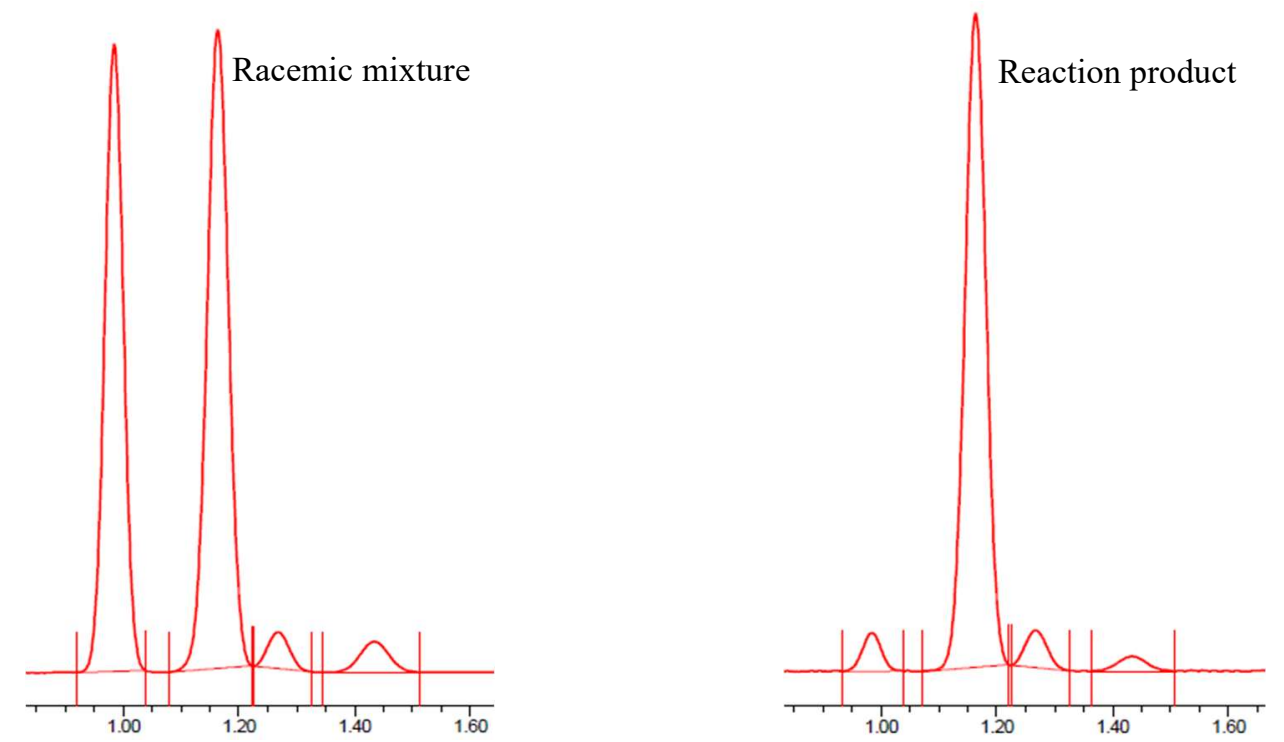

\begin{tabular}{|c|c|c|c|}
\hline $\begin{array}{c}\text { Retention Time } \\
(\mathrm{mins})\end{array}$ & \% Area & $\begin{array}{c}\text { Target } \\
\text { Mass 1 } \\
(\mathrm{Da})\end{array}$ & $\begin{array}{c}\text { Base Peak } \\
(\mathrm{m} / \mathbf{z})\end{array}$ \\
\hline 0.984 & 43.40 & 388.00 & 389.46 \\
\hline 1.163 & 50.51 & 388.00 & 389.45 \\
\hline 1.268 & 2.85 & 388.00 & 389.44 \\
\hline 1.434 & 3.24 & 388.00 & 389.45 \\
\hline
\end{tabular}

\begin{tabular}{|c|c|c|c|}
\hline $\begin{array}{c}\text { Retention Time } \\
(\mathrm{mins})\end{array}$ & \% Area & $\begin{array}{c}\text { Target } \\
\text { Mass 1 } \\
(\mathrm{Da})\end{array}$ & $\begin{array}{c}\text { Base Peak } \\
(\mathrm{m} / \mathbf{z})\end{array}$ \\
\hline 0.984 & 4.53 & 388.00 & 389.46 \\
\hline 1.163 & 87.66 & 388.00 & 389.45 \\
\hline 1.268 & 5.08 & 388.00 & 389.43 \\
\hline 1.433 & 2.74 & 388.00 & 389.48 \\
\hline
\end{tabular}




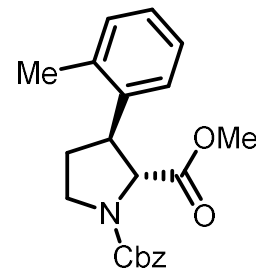

rac-trans-1-benzyl 2-methyl 3-(o-tolyl)pyrrolidine-1,2-dicarboxylate (29). The reaction was performed according to the general procedure $\mathrm{C}$ with $o$-tolylboronic acid $(102 \mathrm{mg}$, $0.75 \mathrm{mmol}, 1.2$ equiv). The crude residue was purified by silica gel chromatography ( 0 $25 \%$ EtOAc in hexanes) to afford the title compound as clear colorless oil (157 mg, 89\% yield, 18:1 dr). ${ }^{1}$ H NMR (600 MHz, DMSO- $\left.d_{6}\right) \delta 7.42-7.37(\mathrm{~m}, 2 \mathrm{H}), 7.37-7.32(\mathrm{~m}$, $1.5 \mathrm{H}), 7.32-7.26(\mathrm{~m}, 2.5 \mathrm{H}), 7.22-7.08(\mathrm{~m}, 3 \mathrm{H}), 5.29-5.10(\mathrm{~m}, 1.5 \mathrm{H}), 5.01(\mathrm{~d}, J=12.7 \mathrm{~Hz}, 0.5 \mathrm{H}), 4.30$ $(\mathrm{d}, J=6.4 \mathrm{~Hz}, 0.5 \mathrm{H}), 4.28(\mathrm{~d}, J=6.2 \mathrm{~Hz}, 0.5 \mathrm{H}), 3.74-3.65(\mathrm{~m}, 2 \mathrm{H}), 3.63(\mathrm{~s}, 1.5 \mathrm{H}), 3.61-3.54(\mathrm{~m}, 1 \mathrm{H})$, $3.52(\mathrm{~s}, 1.5 \mathrm{H}), 2.28(\mathrm{~s}, 1.5 \mathrm{H}), 2.26(\mathrm{~s}, 1.5 \mathrm{H}), 2.24-2.21(\mathrm{~m}, 1 \mathrm{H}), 2.10-1.89(\mathrm{~m}, 1 \mathrm{H}) .{ }^{13} \mathbf{C}$ NMR $(151$ MHz, DMSO- $\left.d_{6}\right) \delta 172.4,172.0,153.9,153.3,138.7,138.6,136.8,136.5,135.6,135.5,130.31,130.28$, 128.4, 128.3, 127.8, 127.7, 127.4, 127.3, 126.8, 126.4, 125.5, 125.3, 66.3, 66.2, 65.5, 65.1, 52.0, 51.9, 46.5, 45.9, 44.9, 43.8, 31.7, 31.0, 19.0. HRESIMS $354.1707 \mathrm{~m} / \mathrm{z}$ (calc'd for $\mathrm{C}_{21} \mathrm{H}_{24} \mathrm{NO}_{4}{ }^{+}, \mathrm{m} / \mathrm{z} 354.1705 \mathrm{~m} / \mathrm{z}$; $\Delta$ ppm 0.6)

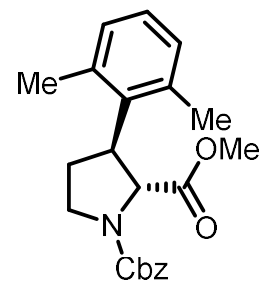

rac-trans-1-benzyl 2-methyl 3-(2,6-dimethylphenyl)pyrrolidine-1,2-dicarboxylate (30). The reaction was performed according to the general procedure $\mathrm{C}$ with $(2,6-$ dimethylphenyl)boronic acid (112 mg, $0.75 \mathrm{mmol}, 1.5$ equiv). The crude residue was purified by silica gel chromatography (0-25\% EtOAc in hexanes) to afford the title compound as clear colorless oil (129 mg, 70\% yield, >20:1 dr). ${ }^{1} \mathbf{H}$ NMR (600 MHz, DMSO- $\left.d_{6}\right) \delta 7.44-7.38(\mathrm{~m}, 2 \mathrm{H}), 7.37$ - $7.32(\mathrm{~m}, 1 \mathrm{H}), 7.32-7.26(\mathrm{~m}, 2 \mathrm{H}), 7.07-6.98(\mathrm{~m}, 3 \mathrm{H}), 5.23-$ $5.10(\mathrm{~m}, 1.5 \mathrm{H}), 5.03(\mathrm{~d}, J=12.7 \mathrm{~Hz}, 0.5 \mathrm{H}), 4.44(\mathrm{~d}, J=8.0 \mathrm{~Hz}, 0.5 \mathrm{H}), 4.42(\mathrm{~d}, J=8.3 \mathrm{~Hz}, 0.5 \mathrm{H}), 3.97-$ $3.91(\mathrm{~m}, 1 \mathrm{H}), 3.91-3.84(\mathrm{~m}, 1 \mathrm{H}), 3.60(\mathrm{~s}, 1.5 \mathrm{H}), 3.51(\mathrm{~s}, 1.5 \mathrm{H}), 3.50-3.43(\mathrm{~m}, 1 \mathrm{H}), 2.28(\mathrm{~s}, 3 \mathrm{H}), 2.27(\mathrm{~s}$, 3H), 2.25 - 2.19 (m, 1H), $2.19-2.12(\mathrm{~m}, 1 \mathrm{H}) .{ }^{13} \mathbf{C}$ NMR (151 MHz, DMSO-d 6 ) $\delta$ 173.1, 172.8, 153.9, 153.3, 136.7, 136.6, 136.2, 135.7, 128.4, 128.3, 127.82, 127.77, 127.4, 127.3, 126.7, 66.3, 62.9, 62.5, 51.9, 47.0, 46.5, 44.7, 43.6, 30.2, 29.4, 20.3. HRESIMS $390.1681 \mathrm{~m} / \mathrm{z}$ (calc'd for $\mathrm{C}_{22} \mathrm{H}_{25} \mathrm{NO}_{4} \mathrm{Na}^{+}$, m/z 390.1681 $\mathrm{m} / \mathrm{z} ; \Delta \mathrm{ppm} 0.3)$

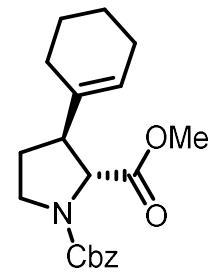

rac-trans-1-benzyl 2-methyl 3-(cyclohex-1-en-1-yl)pyrrolidine-1,2-dicarboxylate (31). The reaction was performed according to general procedure $\mathrm{C}$ with cyclohex-1-en-1ylboronic acid (94 mg, $0.75 \mathrm{mmol}, 1.5$ equiv). The crude residue was purified by silica gel chromatography (0-50\% EtOAc in hexanes) to afford the title compound as clear colorless oil (130.3 mg, 76\% yield, 10:1 dr). ${ }^{1} \mathbf{H}$ NMR (600 MHz, DMSO- $\left.d_{6}\right) \delta 7.41-7.33(\mathrm{~m}, 3 \mathrm{H})$, $7.31(\mathrm{t}, J=6.8 \mathrm{~Hz}, 1 \mathrm{H}), 7.29-7.23(\mathrm{~m}, 1 \mathrm{H}), 5.49(\mathrm{~d}, J=4.1 \mathrm{~Hz}, 1 \mathrm{H}), 5.20-5.04(\mathrm{~m}, 1.5 \mathrm{H}), 4.96(\mathrm{~d}, J=$ $12.7 \mathrm{~Hz}, 0.5 \mathrm{H}), 4.09(\mathrm{~d}, J=6.5 \mathrm{~Hz}, 0.5 \mathrm{H}), 4.07(\mathrm{~d}, J=6.2 \mathrm{~Hz}, 0.5 \mathrm{H}), 3.64(\mathrm{~s}, 1.5 \mathrm{H}), 3.59-3.52(\mathrm{~m}, 1 \mathrm{H})$, $3.52(\mathrm{~s}, 1.5 \mathrm{H}), 3.41(\mathrm{ddt}, J=24.9,10.3,7.4 \mathrm{~Hz}, 1 \mathrm{H}), 2.76(\mathrm{q}, J=7.1 \mathrm{~Hz}, 0.5 \mathrm{H}), 2.72(\mathrm{q}, J=7.2 \mathrm{~Hz}, 0.5 \mathrm{H})$, 
$1.99-1.93(\mathrm{~m}, 5 \mathrm{H}), 1.91-1.78(\mathrm{~m}, 1 \mathrm{H}), 1.56(\mathrm{~d}, J=5.0 \mathrm{~Hz}, 2 \mathrm{H}), 1.50(\mathrm{~h}, J=5.9 \mathrm{~Hz}, 2 \mathrm{H}) .{ }^{13} \mathbf{C} \mathbf{N M R}(151$ MHz, DMSO- $\left.d_{6}\right) \delta 172.5,172.2,153.8,153.3,136.8,136.6,135.5,135.4,128.3,128.2,127.8,127.7$, $127.40,127.38,127.3,122.3,122.1,66.2,66.1,62.5,62.0,51.8,51.8,51.1,49.9,46.2,45.7,29.0,28.2$, 25.5, 25.4, 24.6, 22.3, 21.8. HRESIMS $344.1875 \mathrm{~m} / \mathrm{z}$ (calc'd for $\mathrm{C}_{20} \mathrm{H}_{26} \mathrm{NO}_{4}{ }^{+}, \mathrm{m} / \mathrm{z} 344.1862 \mathrm{~m} / \mathrm{z} ; \Delta \mathrm{ppm}$ $3.8)$

\section{DFT calculations and additional experiments}

DFT Calculations: cis-2 and trans-2 were subjected to a thorough mixed torsional/low-mode conformational sampling in Maestro (Schrödinger Release 2020-2: Maestro, Schrödinger, LLC, New York, NY, 2020.) for a maximum number of 100000 steps with a window for saving conformations of $10 \mathrm{kcal} / \mathrm{mol}$ and a $0.5 \AA$ RMSD cutoff. 97 Low energy conformers were found for cis-2 and 243 conformers for trans2. Each conformer was subjected to DFT geometry optimizations and frequency calculations (Gaussian 16, Revision B.01, Gaussian, Inc., Wallingford CT, 2016.) M06-2X/6-31+G** level. $\Delta \mathrm{G}$ between trans-2 and cis-2 was found to be $0.183 \mathrm{kcal} / \mathrm{mol}$ favoring trans-2.

Additional Experiments:

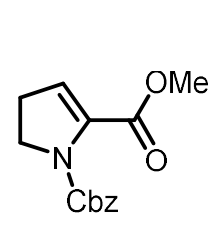

1

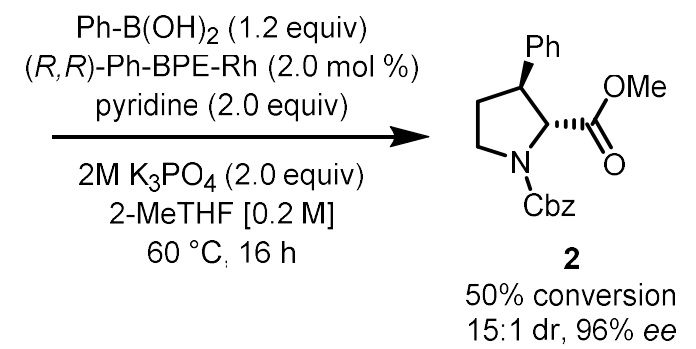

Reaction with addition of pyridine: The reaction was performed according to general procedure A with phenylboronic acid (73 mg, $0.6 \mathrm{mmol}, 1.2$ equiv), except pyridine ( $81 \mu \mathrm{L}, 1.0 \mathrm{mmol}, 2.0$ equiv) was also added after the addition of 2-MeTHF in the glove box. Crude ${ }^{1} \mathrm{H}$ NMR after stirring for $16 \mathrm{~h}$ at $60{ }^{\circ} \mathrm{C}$ followed by standard work-up indicated 1:1 ratio of 1:2 (15:1 dr). SFC indicated 96\% ee of trans-2. 

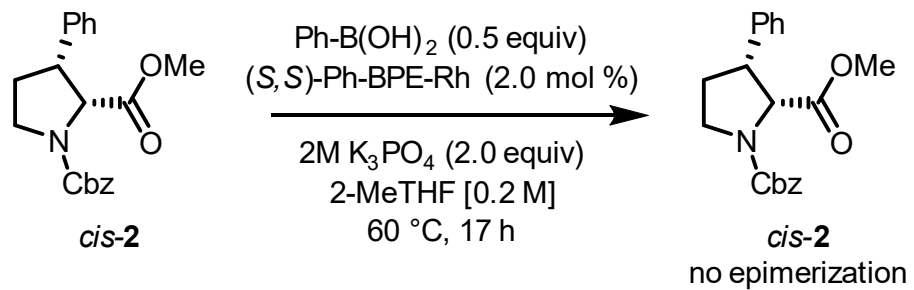

Subjecting cis-2 to the reaction conditions: The reaction was performed according to general procedure A with $(S, S)$-Ph-BPE-Rh but instead of substrate 1, cis-2 $(7 \mathrm{mg}, 0.02 \mathrm{mmol})$ was added to the reaction mixture. The reaction was stirred for $17 \mathrm{~h}$ at $60{ }^{\circ} \mathrm{C}$. After standard work-up, only cis-2 was recovered and no trans2 was observed $\left({ }^{1} \mathrm{H} \mathrm{NMR}, \mathrm{CHCl}_{3}, 600.13 \mathrm{MHz}\right)$.

Segment of ${ }^{1} \mathrm{H}$ NMR of starting material

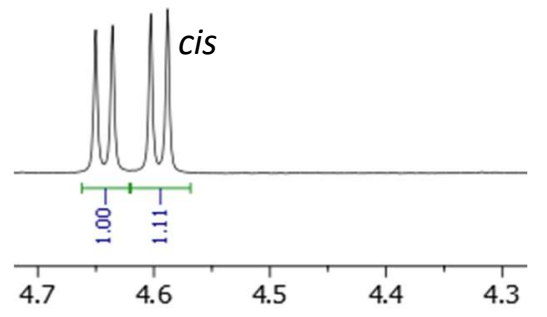

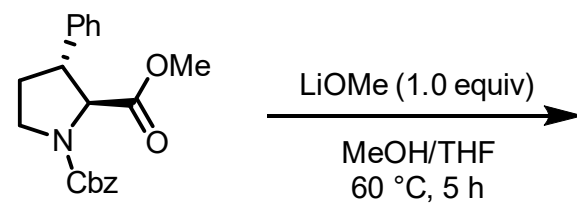

trans-2
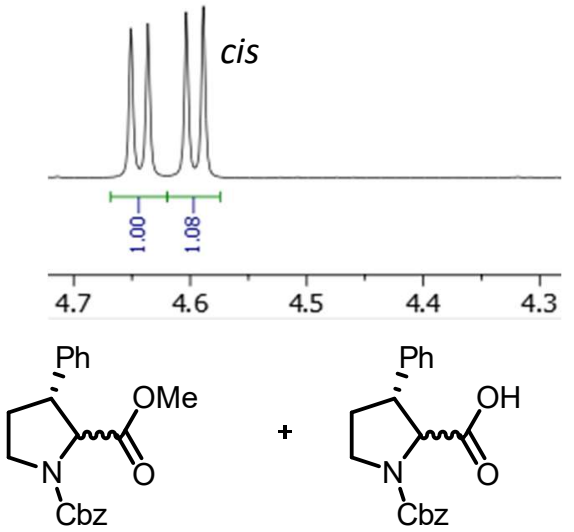

S-7

Epimerization experiment: trans-2 $(50 \mathrm{mg}, 0.147 \mathrm{mmol})$ was added to a 2-dram vial equipped with a septa cap and a stir bar and purged with $\mathrm{N}_{2}$. Anhydrous THF $(0.5 \mathrm{~mL})$ and methanol $(0.5 \mathrm{~mL})$ were added followed by LiOMe $(5.59 \mathrm{mg}, 0.147 \mathrm{mmol})$. The vial was sealed and heated to $60{ }^{\circ} \mathrm{C}$ for $5 \mathrm{~h}$. LCMS indicated a 65:35 LCAP ratio of $\mathbf{2}$ to S-7. The crude mixture was allowed to cool to rt and diluted with $\mathrm{CHCl}_{3}$ and water. The layers were separated and the aq. layer was extracted $3 \mathrm{x}$ with $\mathrm{CHCl}_{3}$. The combined organic layers were dried over $\mathrm{MgSO}_{4}$, filtered, and concentrated under reduced pressure. Crude ${ }^{1} \mathrm{H}$ NMR $\left(\mathrm{CHCl}_{3}, 600.13 \mathrm{MHz}\right)$ indicated a $4.2: 1 \mathrm{dr}$ of 2.

Segment of ${ }^{1} \mathrm{H}$ NMR of starting material

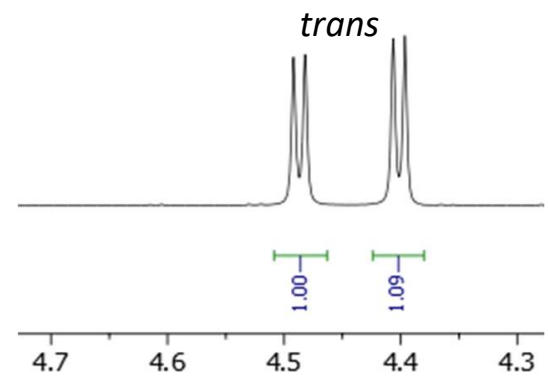

Segment of ${ }^{1} \mathrm{H}$ NMR of product

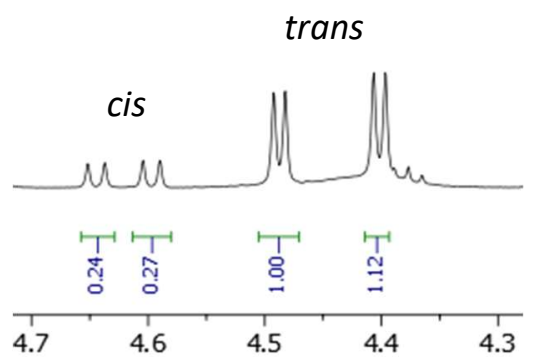




\section{References}

${ }^{1}$ Huy, P.; Neudörfl, J.-M.; Schmalz, H.-G. A Practical Synthesis of Trans-3-Substituted Proline Derivatives through 1,4-Addition. Org. Lett., 2011, 13, 216-219.

${ }^{2}$ Tanemasa, N.; Hideki, K. A New and Convenient Method for the Synthesis of Dehydroamino Acids Starting from Ethyl N-Boc- and N-Z- $\alpha$-Tosylglycinates and Various Nitro Compounds. Bull. Chem. Soc. Jpn. 2000, 73, 1605-

1613.

${ }^{3}$ Switzer, F. L.; van Halbeek, H.; Holt, E. M.; Stammer, C. H. Synthesis of ( \pm )-2,3-Methanoproline: A Novel Inhibitor of Ethylene Biosynthesis. Tetrahedron, 1989, 45, 6091-6100.

${ }^{4}$ Jain, A. N.; Cleves, A. E.; Gao, Q.; Wang, X.; Liu, Y.; Sherer, E. C.; Reibarkh, M. Y., Complex Macrocycle Exploration: Parallel, Heuristic, and Constraint-Based Conformer Generation Using ForceGen. J. Comput. Aided Mol. Des. 2019, 33, 531-558

${ }^{5}$ Frisch, M. J.; Trucks, G. W.; Schlegel, H. B.; Scuseria, G. E.; Robb, M. A.; Cheeseman, J. R.; Scalmani, G.; Barone, V.; Petersson, G. A.; Nakatsuji, H.; Li, X.; Caricato, M.; Marenich, A. V.; Bloino, J.; Janesko, B. G.; Gomperts, R.; Mennucci, B.; Hratchian, H. P.; Ortiz, J. V.; Izmaylov, A. F.; Sonnenberg, J. L.; Williams; Ding, F.; Lipparini, F.; Egidi, F.; Goings, J.; Peng, B.; Petrone, A.; Henderson, T.; Ranasinghe, D.; Zakrzewski, V. G.; Gao, J.; Rega, N.; Zheng, G.; Liang, W.; Hada, M.; Ehara, M.; Toyota, K.; Fukuda, R.; Hasegawa, J.; Ishida, M.; Nakajima, T.; Honda, Y.; Kitao, O.; Nakai, H.; Vreven, T.; Throssell, K.; Montgomery Jr., J. A.; Peralta, J. E.; Ogliaro, F.; Bearpark, M. J.; Heyd, J. J.; Brothers, E. N.; Kudin, K. N.; Staroverov, V. N.; Keith, T. A.; Kobayashi, R.; Normand, J.; Raghavachari, K.; Rendell, A. P.; Burant, J. C.; Iyengar, S. S.; Tomasi, J.; Cossi, M.; Millam, J. M.; Klene, M.; Adamo, C.; Cammi, R.; Ochterski, J. W.; Martin, R. L.; Morokuma, K.; Farkas, O.; Foresman, J. B.; Fox, D. J. Gaussian 16 Rev. B.01: Wallingford, CT, 2016.

${ }^{6}$ Pescitelli, G.; Bruhn, T., Good Computational Practice in the Assignment of Absolute Configurations by TDDFT Calculations of ECD Spectra. Chirality 2016, 28, 466-474.

${ }^{7}$ Mandelare, P. E.; Adpressa, D. A.; Kaweesa, E. N.; Zakharov, L. N.; Loesgen, S., Coculture of Two Developmental Stages of a Marine-Derived Aspergillus alliaceus Results in the Production of the Cytotoxic Bianthrone Allianthrone A. J. Nat. Prod. 2018, 81, 1014-1022.

${ }^{8}$ Neuhaus, G. F.; Adpressa, D. A.; Bruhn, T.; Loesgen, S., Polyketides from Marine-Derived Aspergillus porosus: Challenges and Opportunities for Determining Absolute Configuration. J. Nat. Prod. 2019, 82, 2780-2789.

${ }^{9}$ Harder, E.; Damm, W.; Maple, J.; Wu, C.; Reboul, M.; Xiang, J. Y.; Wang, L.; Lupyan, D.; Dahlgren, M. K.; Knight, J. L.; Kaus, J. W.; Cerutti, D. S.; Krilov, G.; Jorgensen, W. L.; Abel, R.; Friesner, R. A., OPLS3: A Force Field Providing Broad Coverage of Drug-like Small Molecules and Proteins. J. Chem. Theory Comput. 2016, 12, 281-296.

${ }^{10}$ Bruhn, T.; Schaumlöffel, A.; Hemberger, Y.; Bringmann, G., SpecDis: Quantifying the Comparison of Calculated and Experimental Electronic Circular Dichroism Spectra. Chirality 2013, 25, 243-249. 


\section{NMR Spectra}

${ }^{1} \mathrm{H} \mathrm{NMR}-600.13 \mathrm{MHz}-\mathrm{CDCl}_{3} @ 308.0 \mathrm{~K}$<smiles>O=C(OCc1ccccc1)C1=CCCN1C(=O)[O-]</smiles>

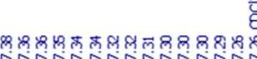

S-3

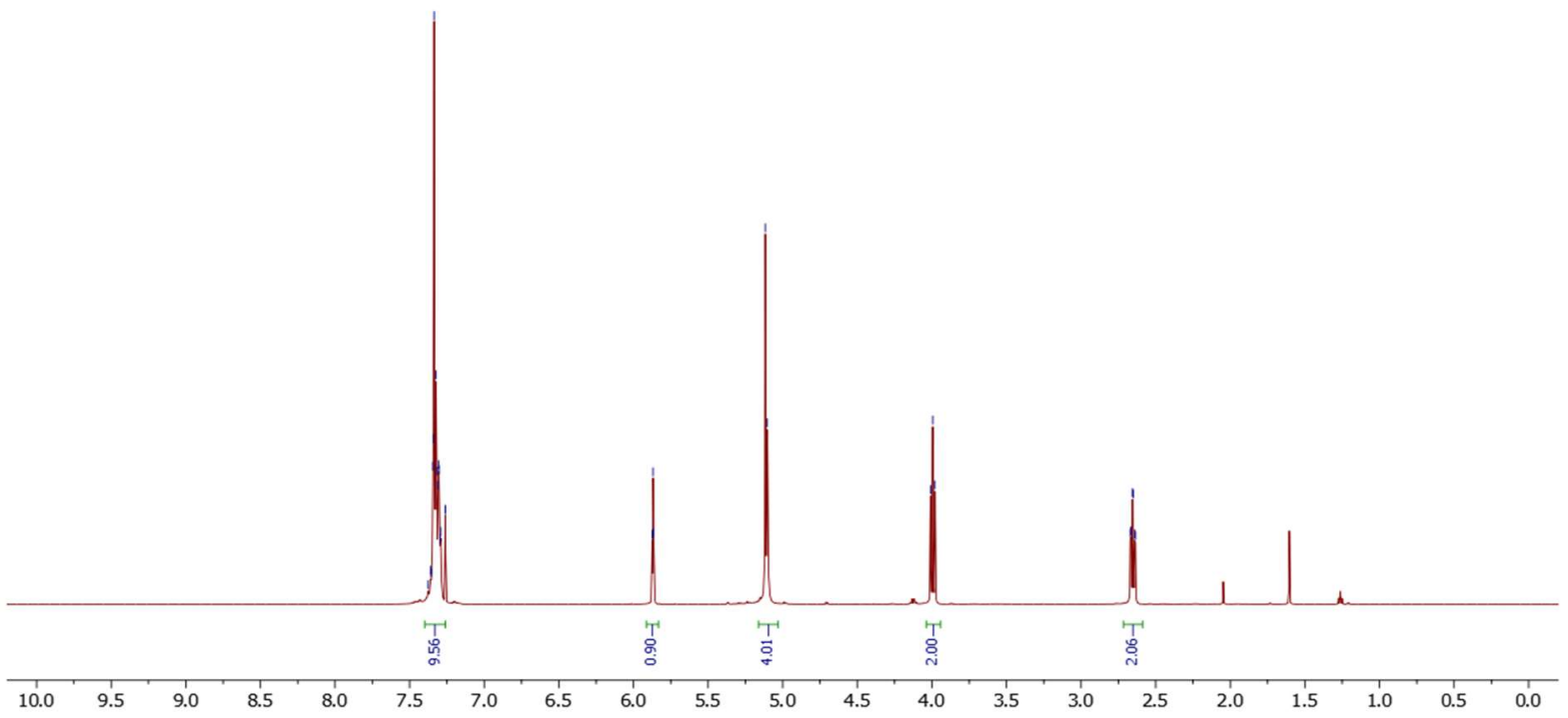

${ }^{13} \mathrm{C}$ NMR - $150.92 \mathrm{MHz}$ - DMSO-d6@308.0 K

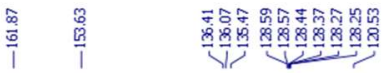
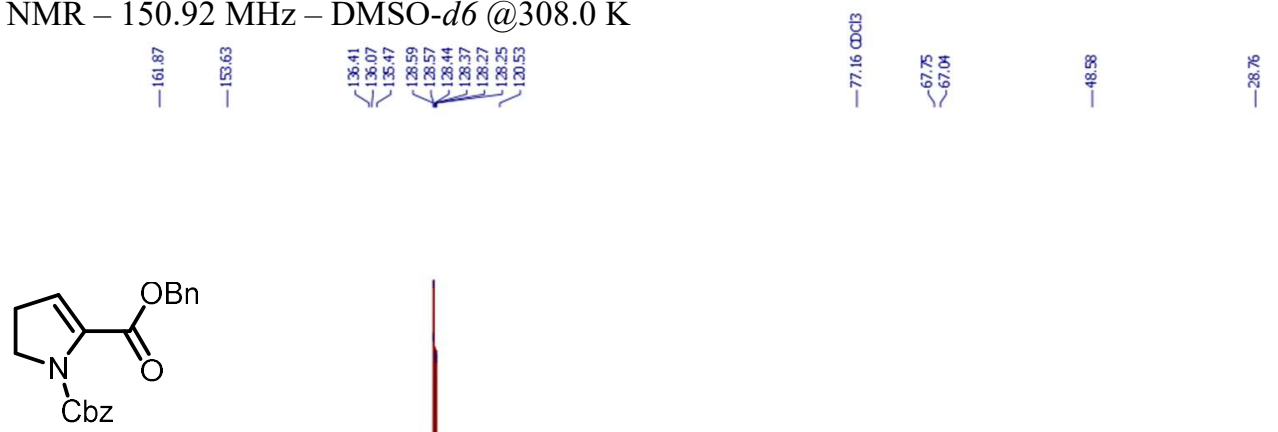

S-3

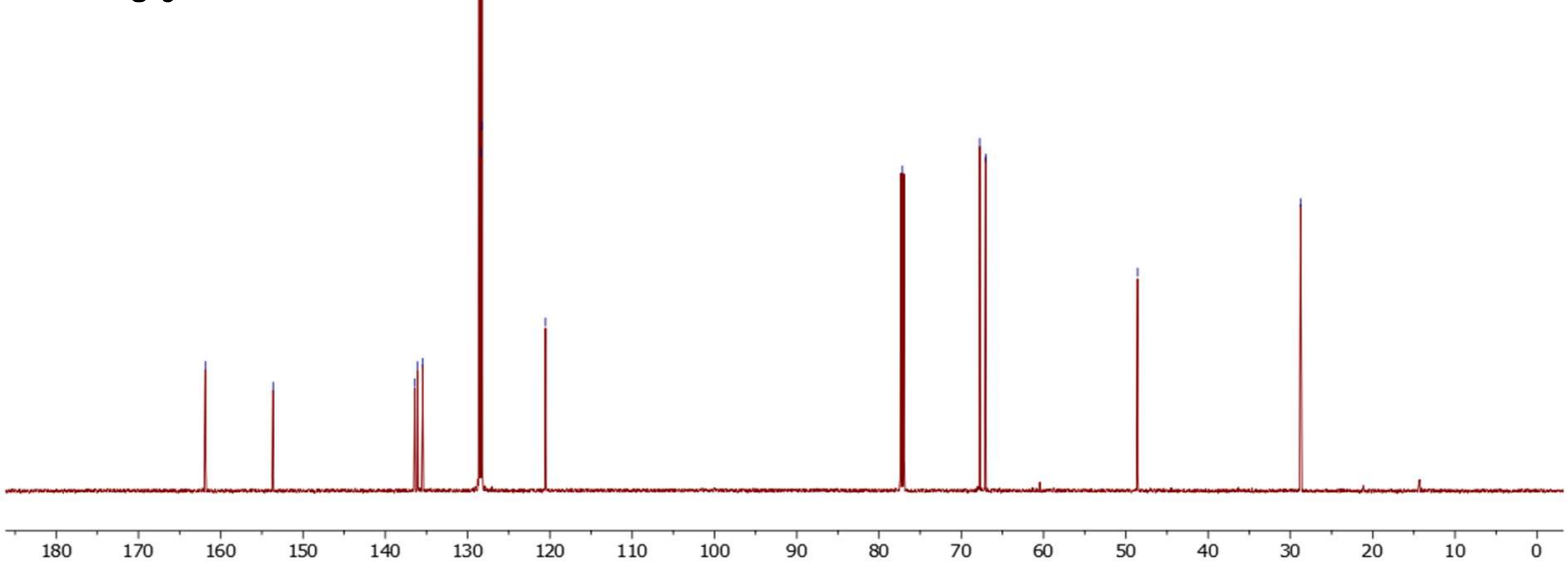


${ }^{1} \mathrm{H} \mathrm{NMR}-600.13 \mathrm{MHz}-\mathrm{CDCl}_{3} @ 308.0 \mathrm{~K}$
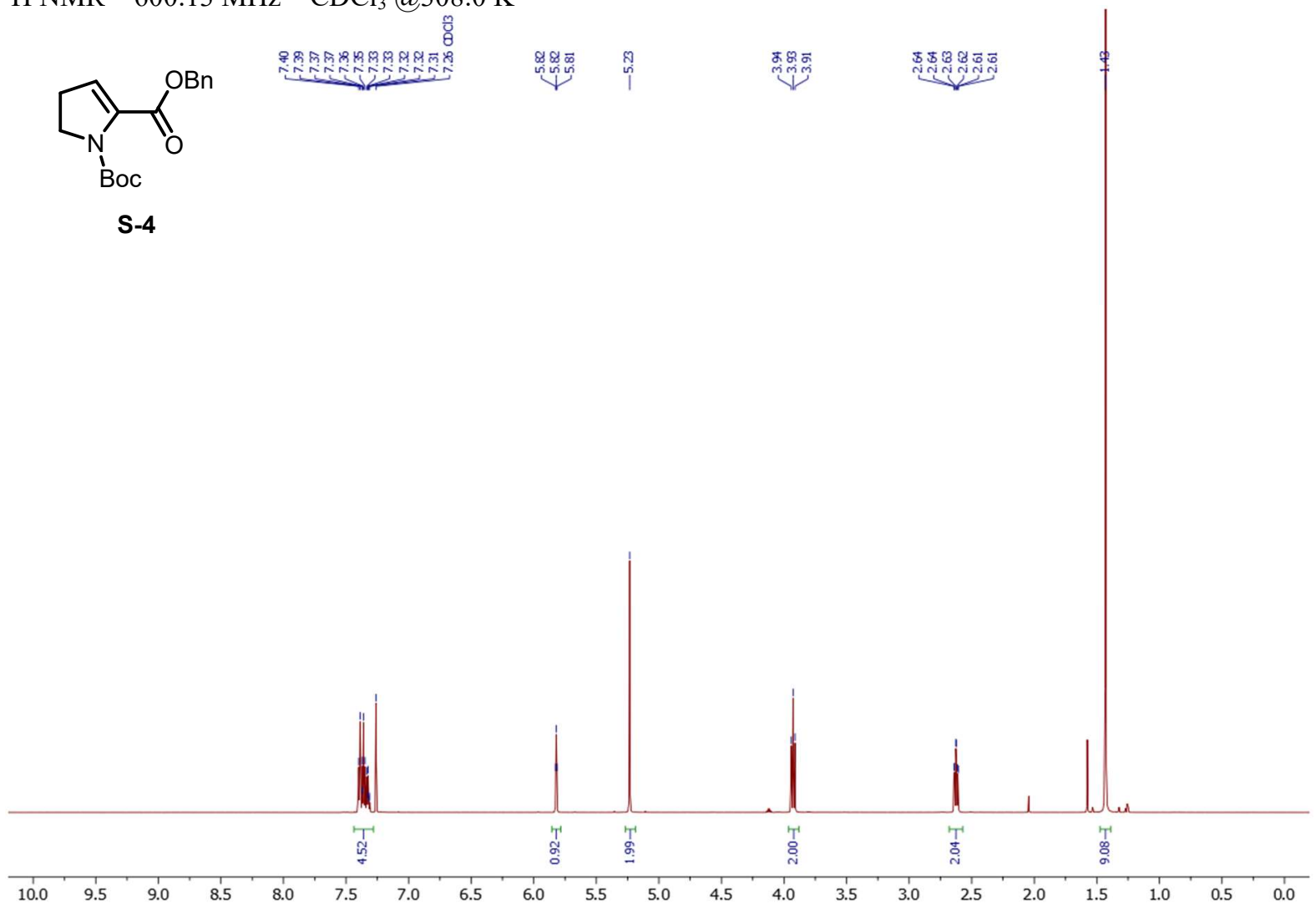

${ }^{13} \mathrm{C}$ NMR - $150.92 \mathrm{MHz}$ - DMSO-d6 @308.0 K

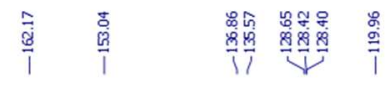
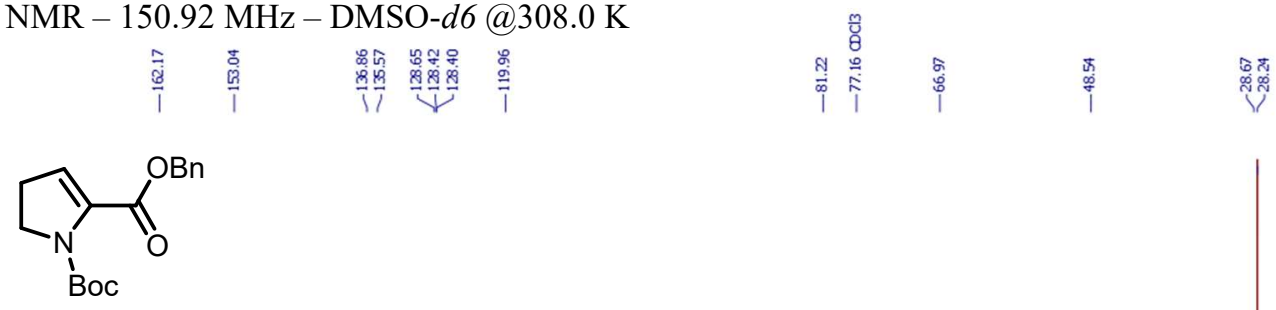

S-4

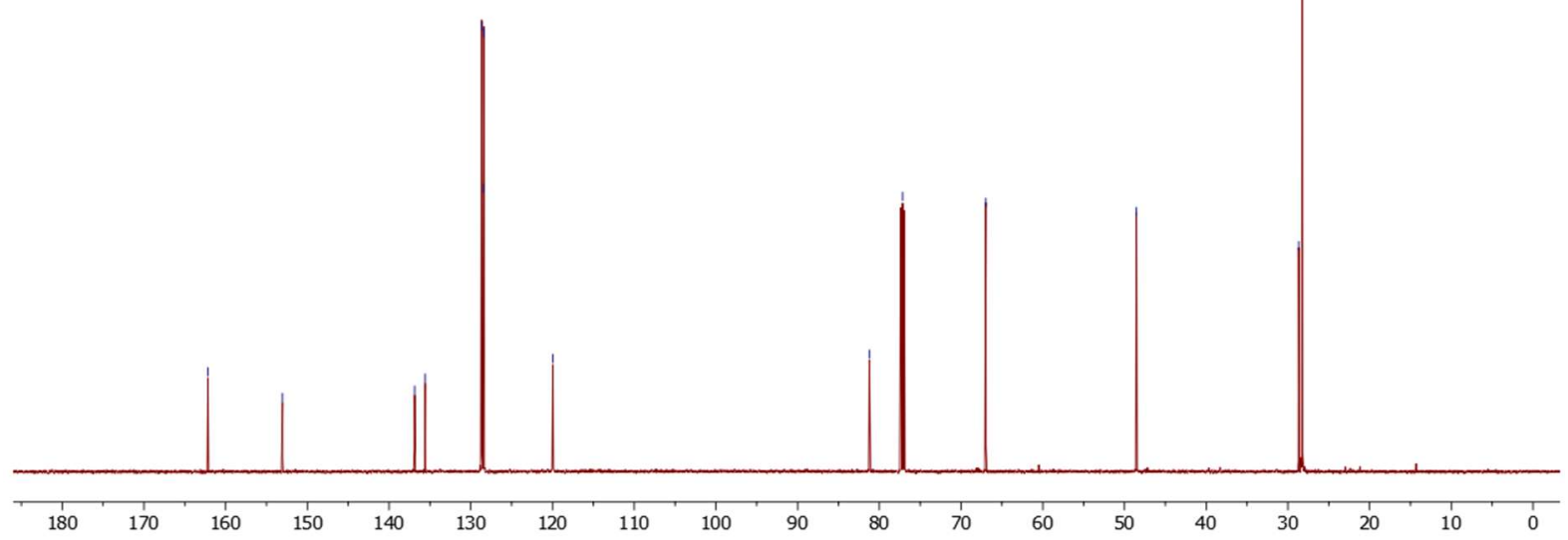




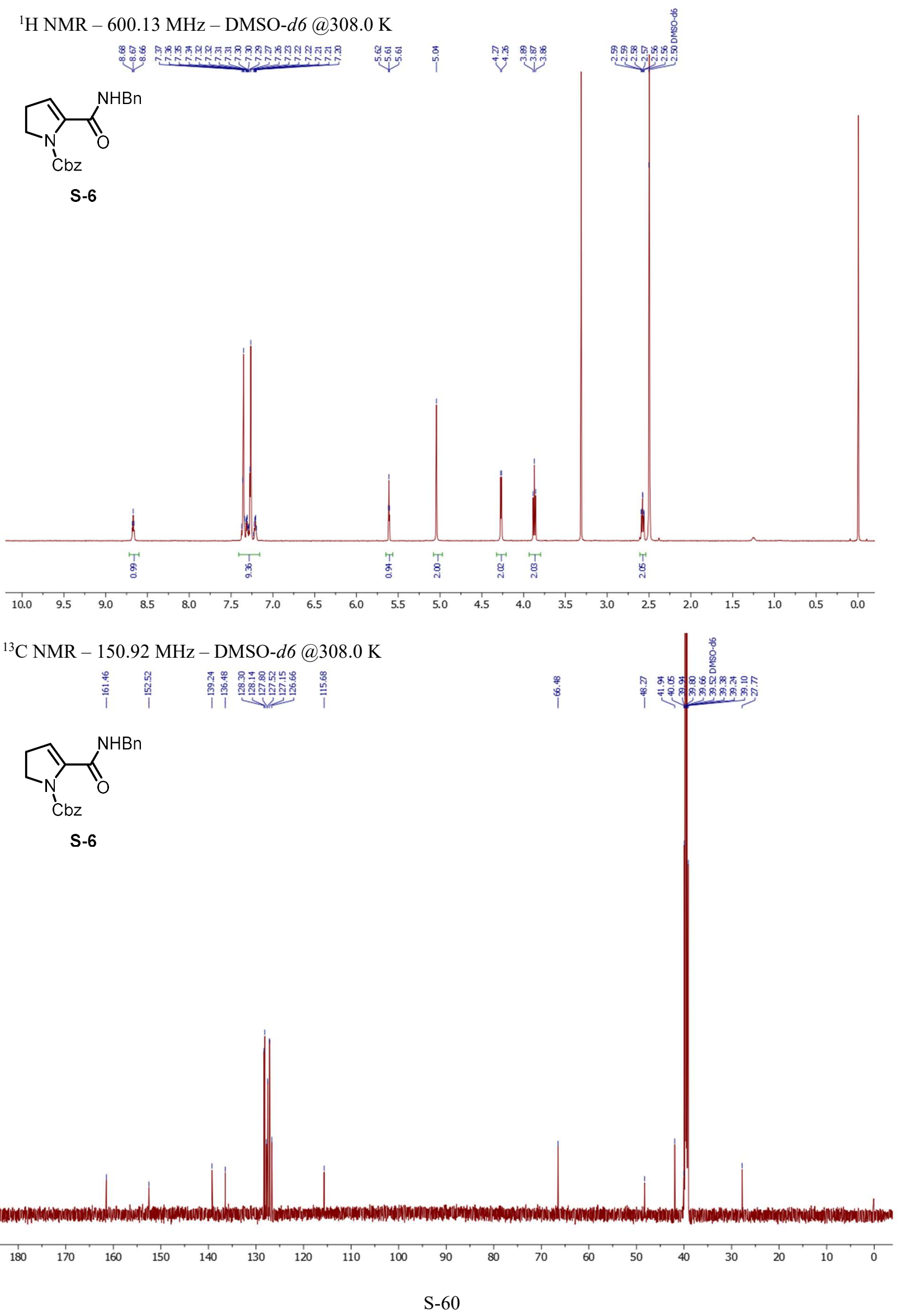


${ }^{1} \mathrm{H}$ NMR - 600.13 MHz - DMSO-d6@308.0 K<smiles>COC(=O)[C@H]1[C@@H](c2ccccc2)CCN1C(=O)OCc1ccccc1</smiles>

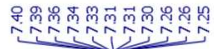

trans-2

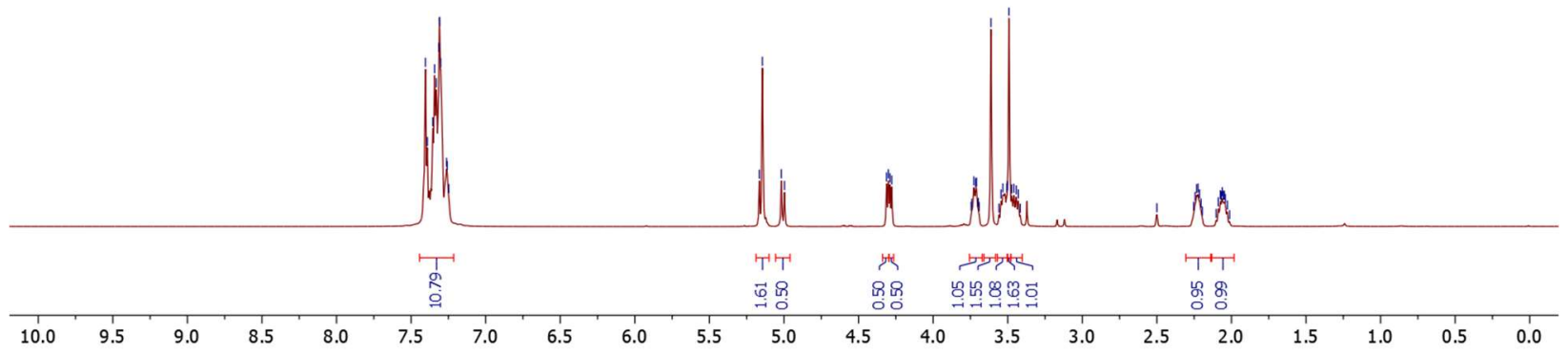

${ }^{13} \mathrm{C}$ NMR - 150.92 MHz - DMSO-d6@308.0 K
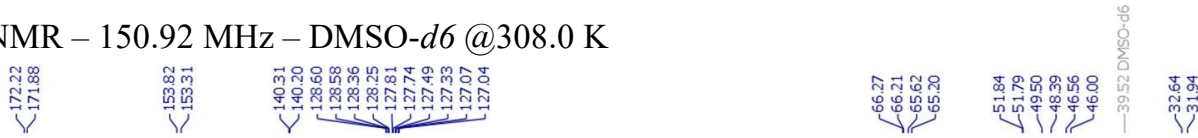<smiles>COC(=O)[C@H]1[C@@H](c2ccccc2)CCN1C(=O)OCc1ccccc1</smiles>

trans-2
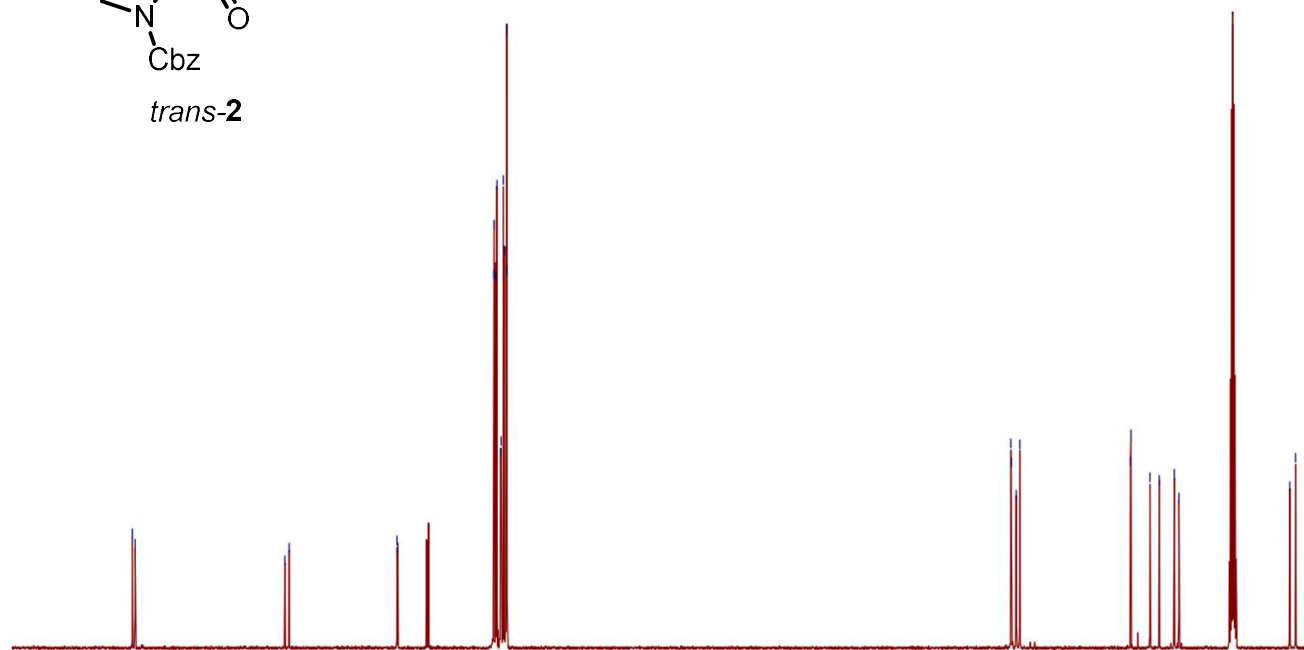

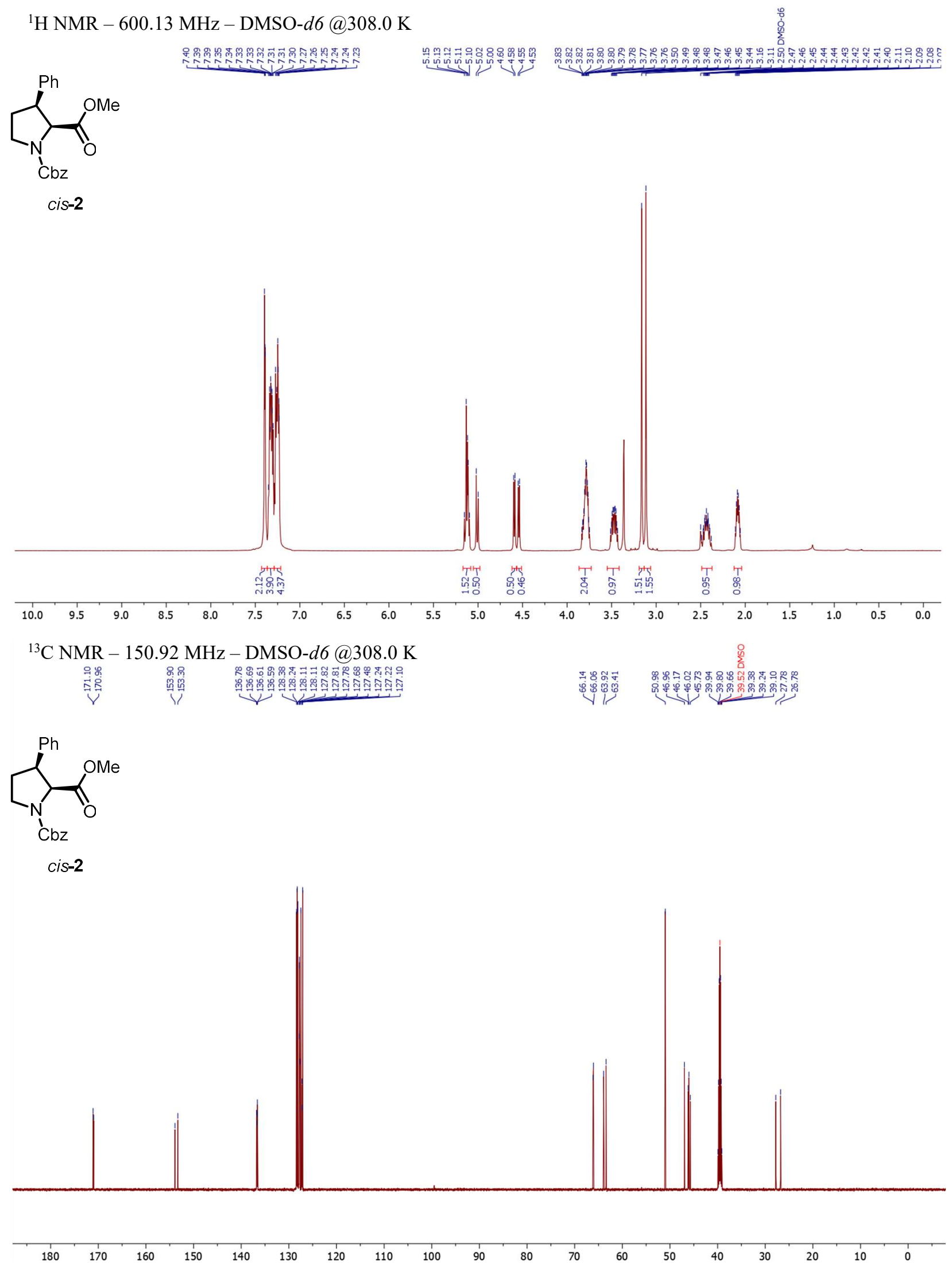
${ }^{1} \mathrm{H}$ NMR - 600.13 MHz - DMSO-d6@308.0 K

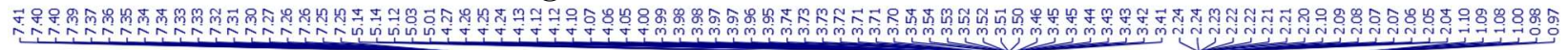<smiles>CCOC(=O)[C@H]1[C@@H](c2ccccc2)CCN1C(=O)OCc1ccccc1</smiles>

3

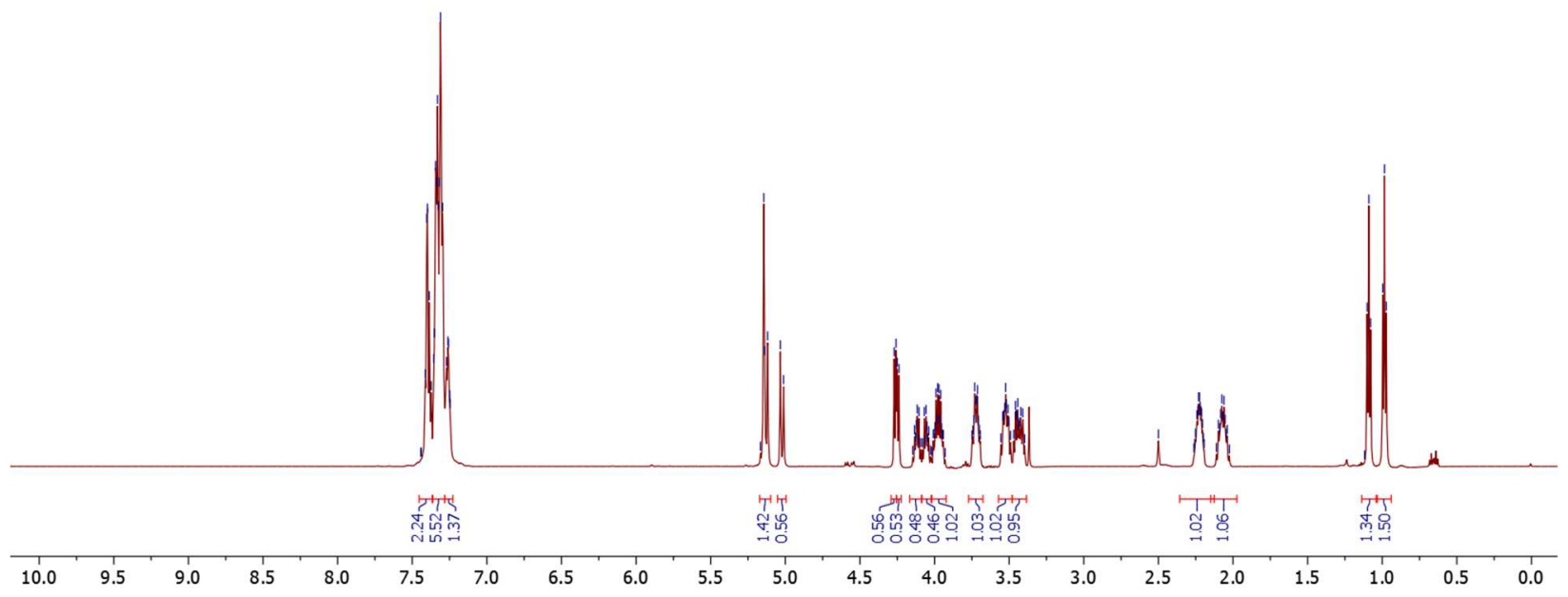

${ }^{13} \mathrm{C}$ NMR - 150.92 MHz - DMSO-d6@308.0 K

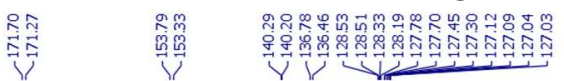<smiles>CCOC(=O)[C@@H]1[C@@H](c2ccccc2)CCN1C(=O)OCc1ccccc1</smiles>

3

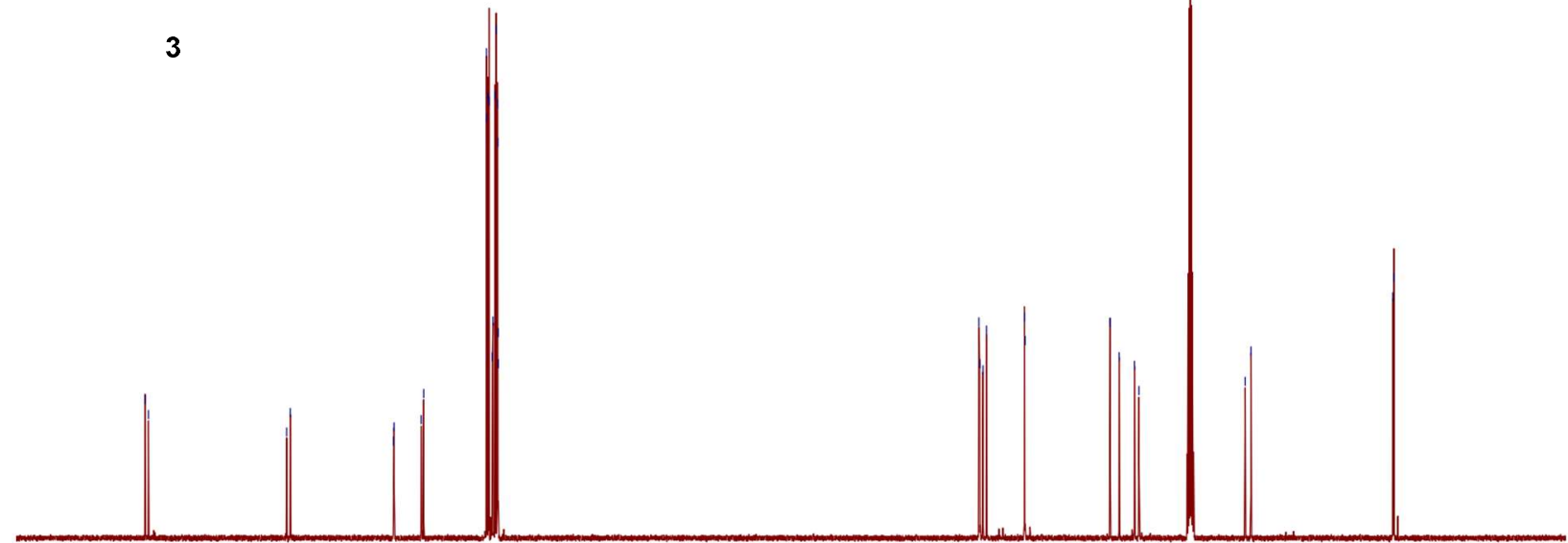

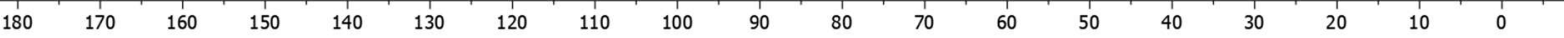


${ }^{1} \mathrm{H}$ NMR - 600.13 MHz - DMSO-d6@308.0 K<smiles>CCCOC(=O)[C@@H]1[C@@H](c2ccccc2)CCN1C(=O)OCc1ccccc1</smiles>

4

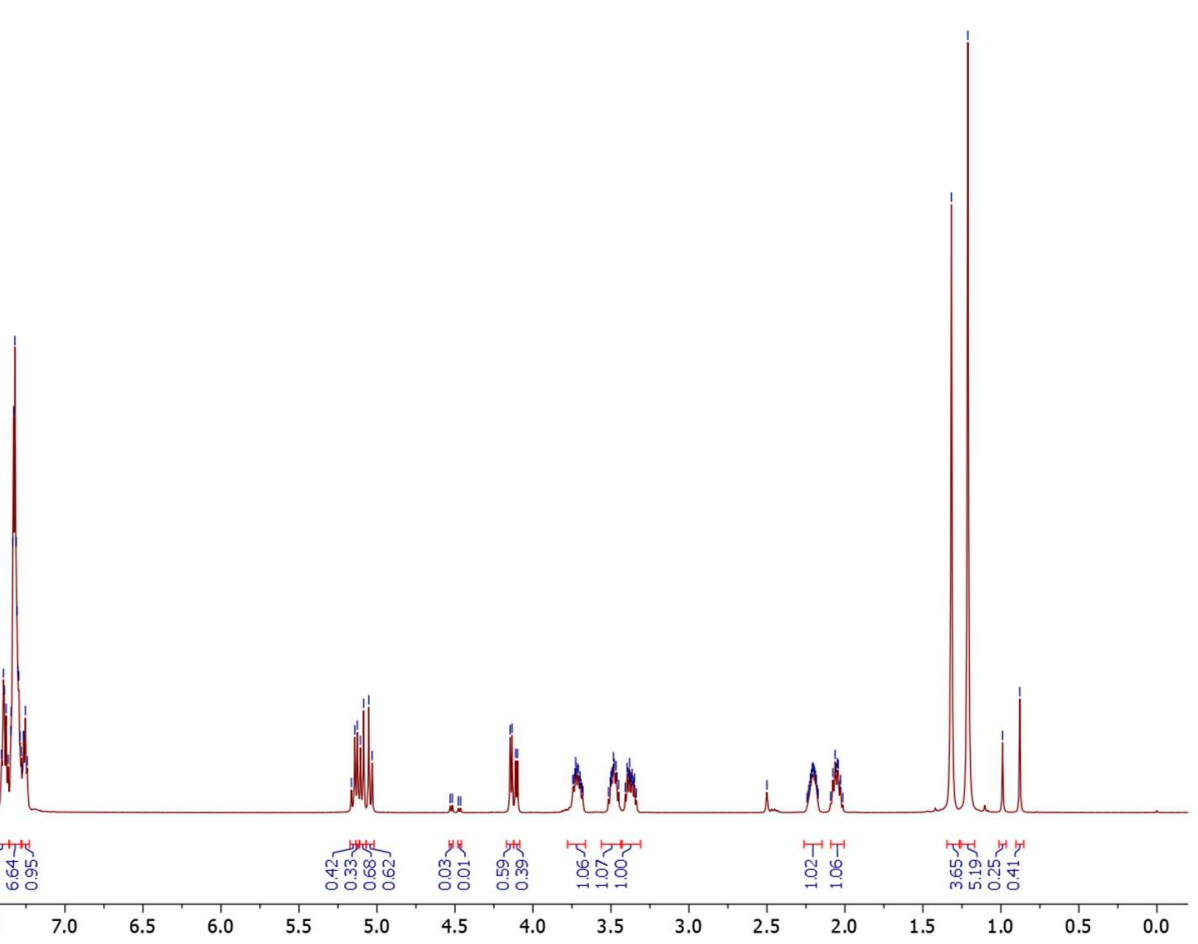

${ }^{13} \mathrm{C}$ NMR - 150.92 MHz - DMSO-d6@308.0 K

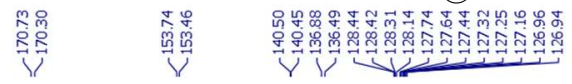

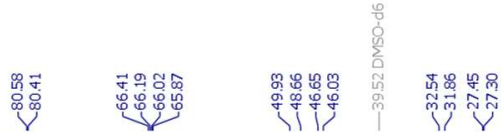<smiles>CCCCC(=O)OC1[C@H](c2ccccc2)CCN1C(=O)[O-]</smiles>

4
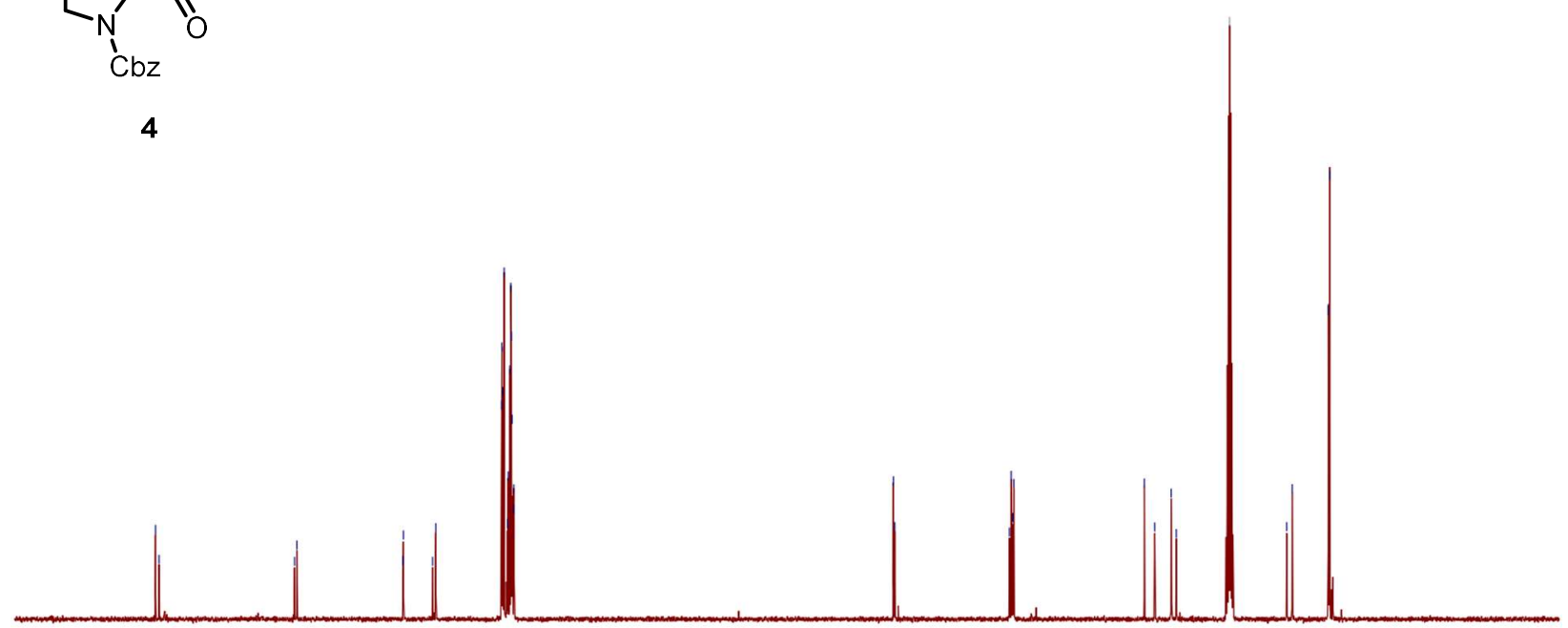

$180-170-160-150-140-130$

$+120,110,100,90$

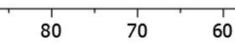

$50+40$

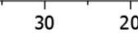


${ }^{1} \mathrm{H}$ NMR - 600.13 MHz - DMSO-d6@308.0 K

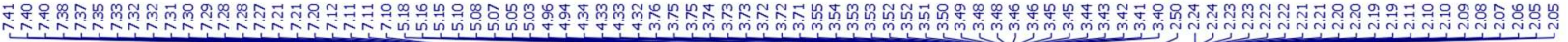<smiles>O=C(OCc1ccccc1)[C@@H]1[C@H](OCc2ccccc2)CCN1C(=O)O</smiles>

5

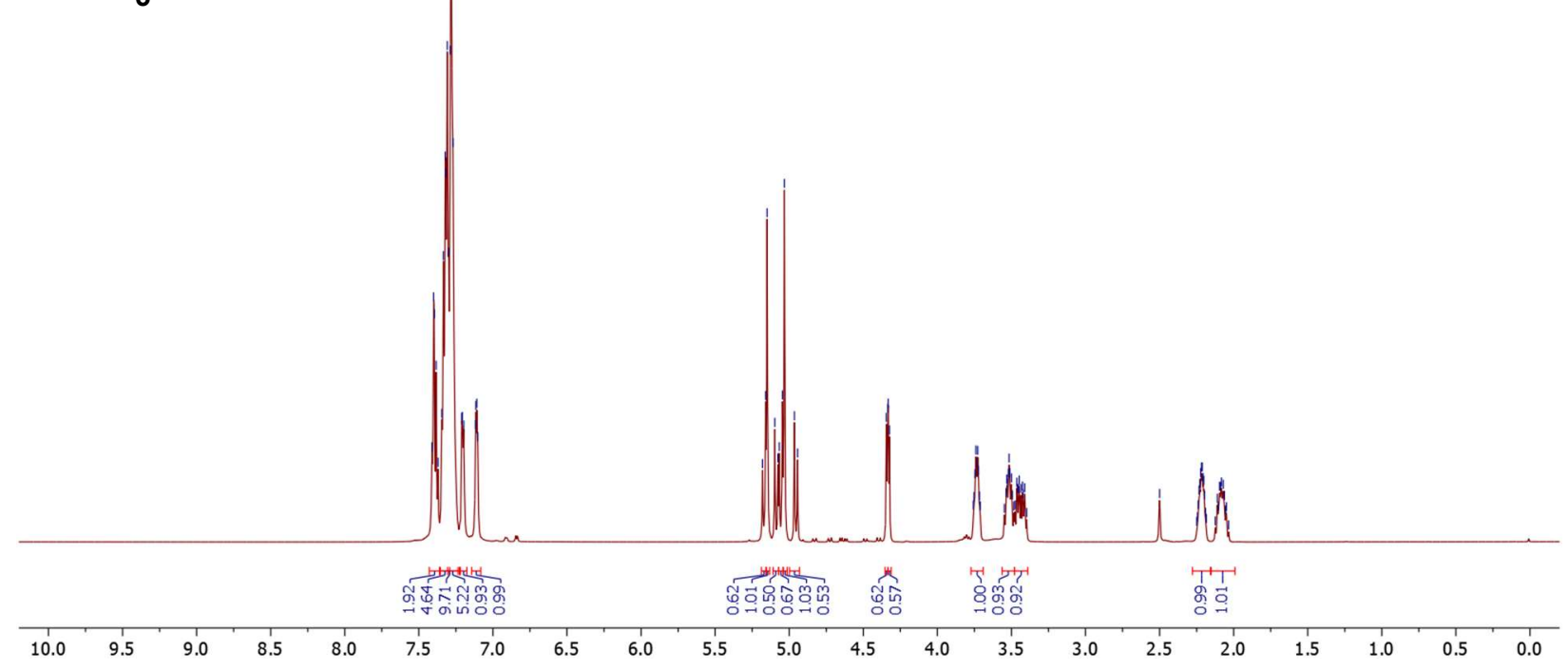

${ }^{13} \mathrm{C}$ NMR - 150.92 MHz - DMSO-d6@308.0 K

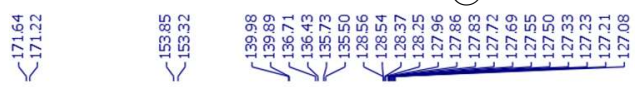

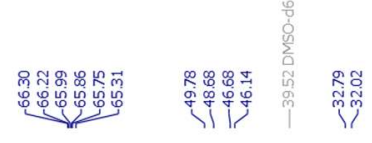<smiles>O=C(Cc1ccccc1)C1[C@H](C(=O)O)CCN1C(=O)c1ccccc1</smiles>

5
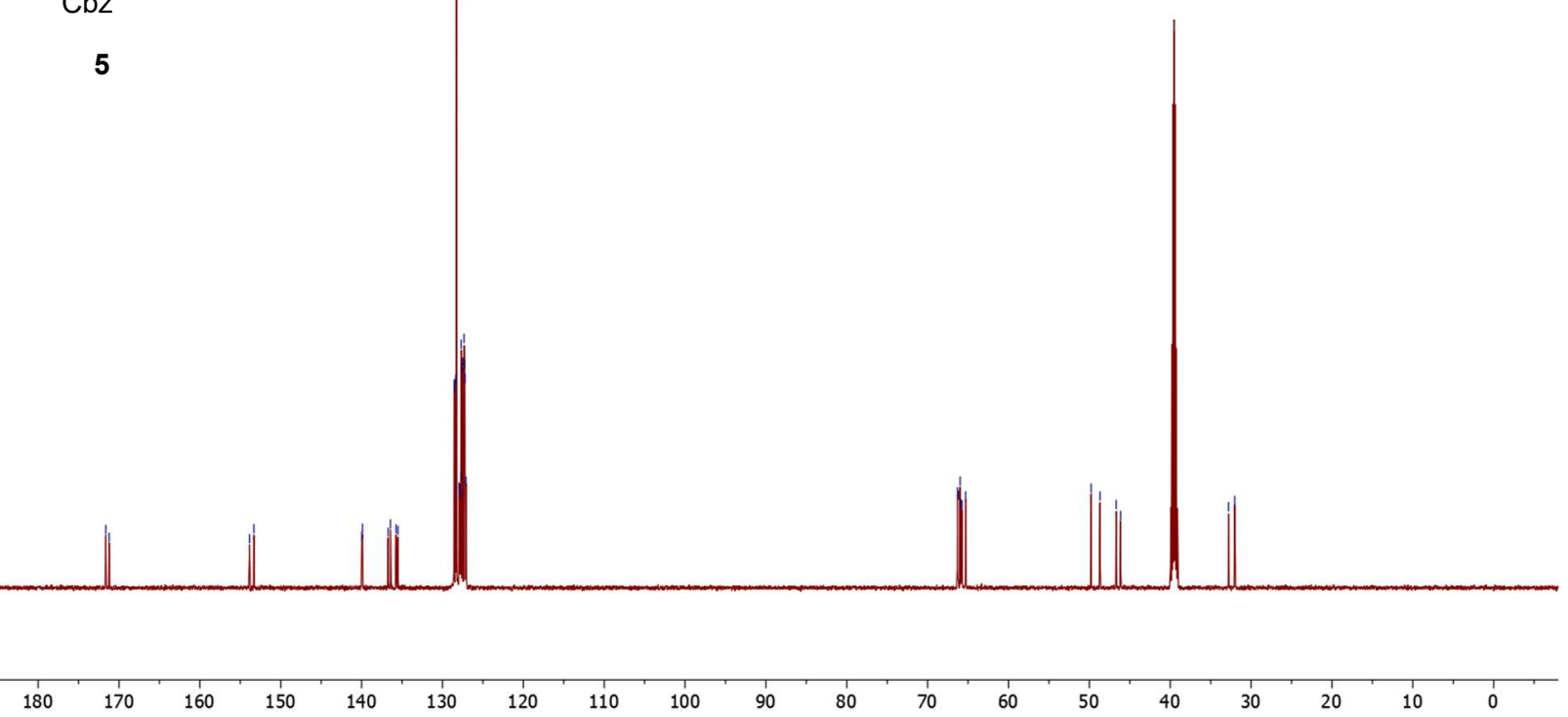


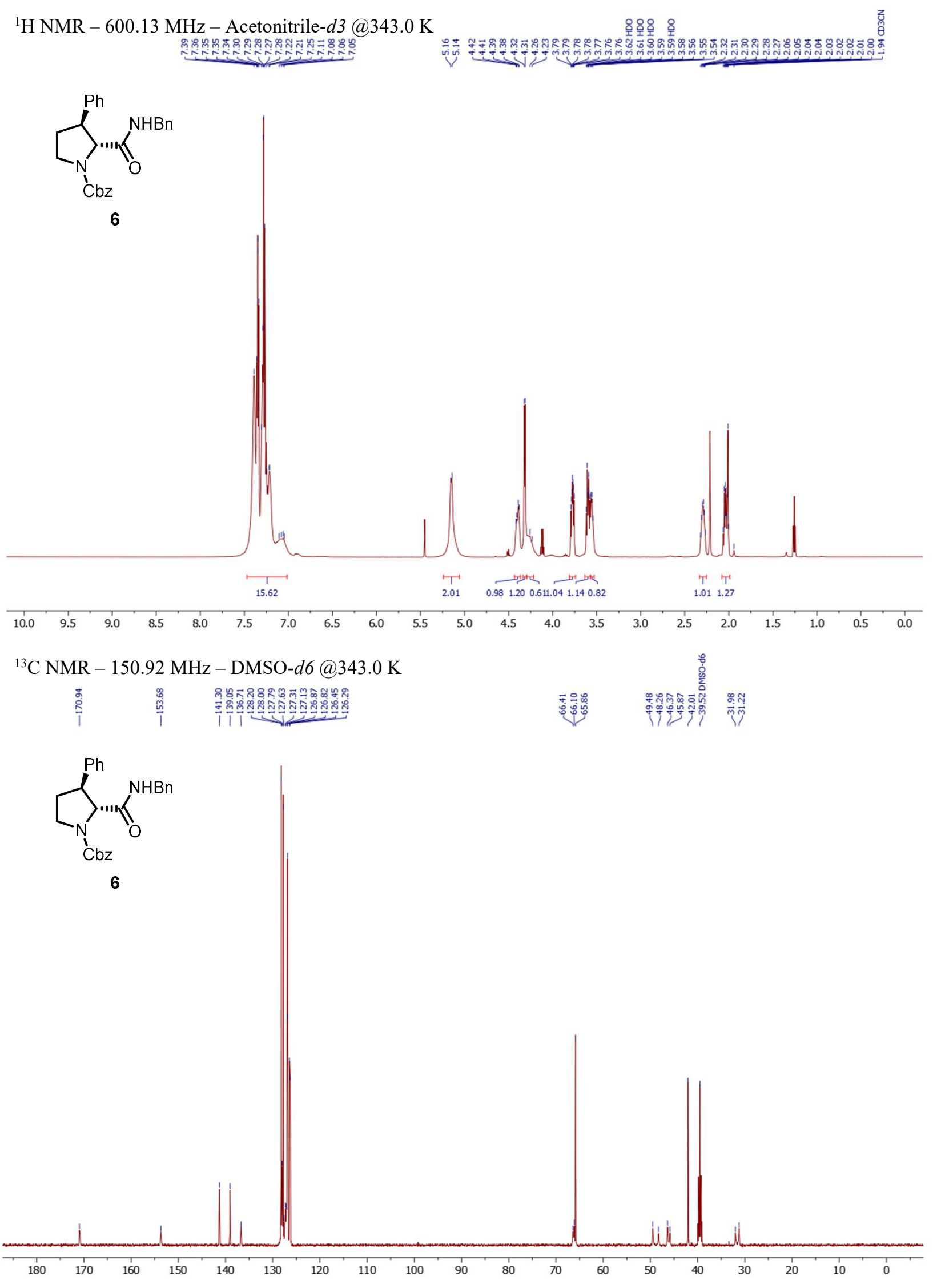



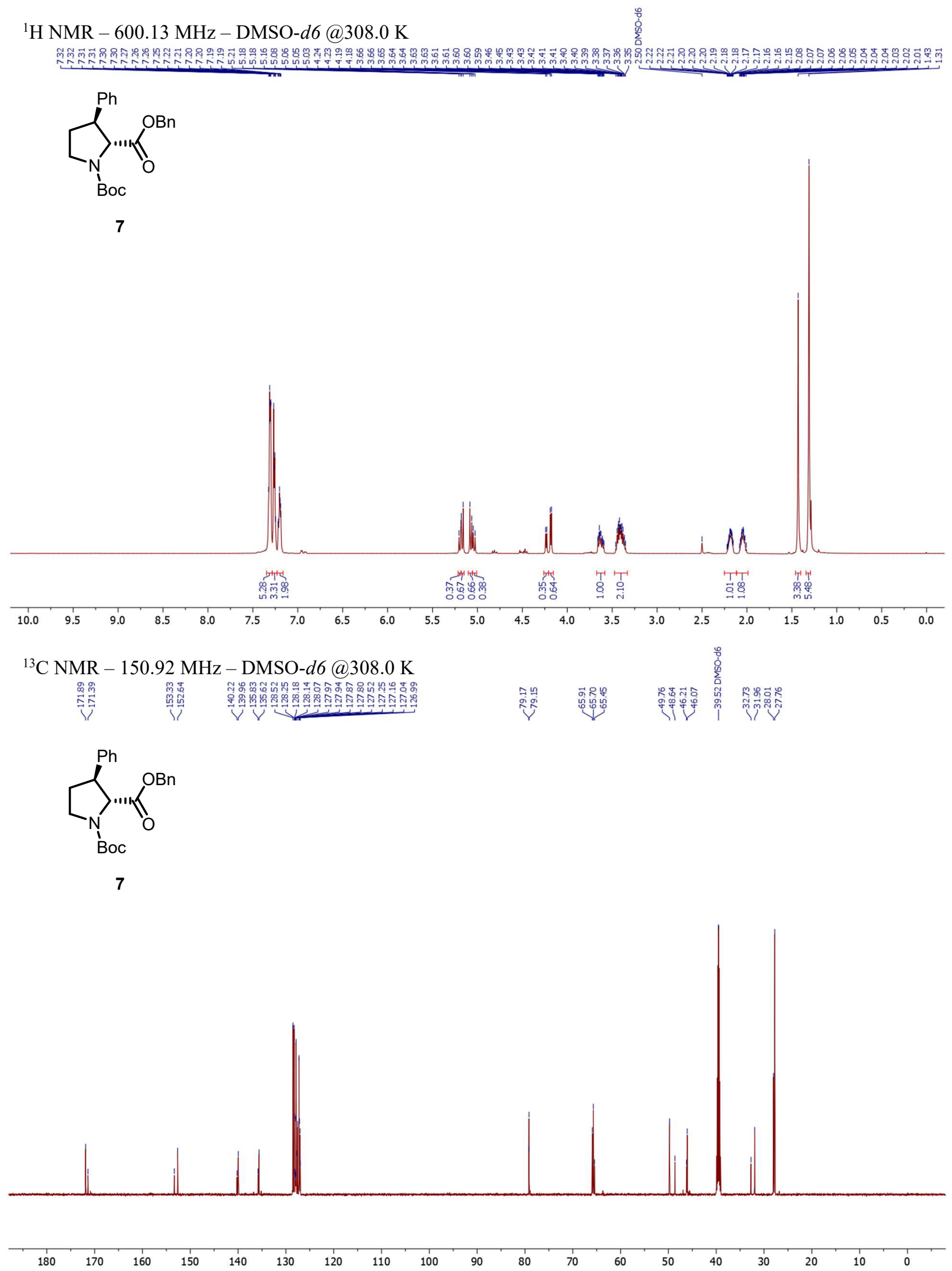


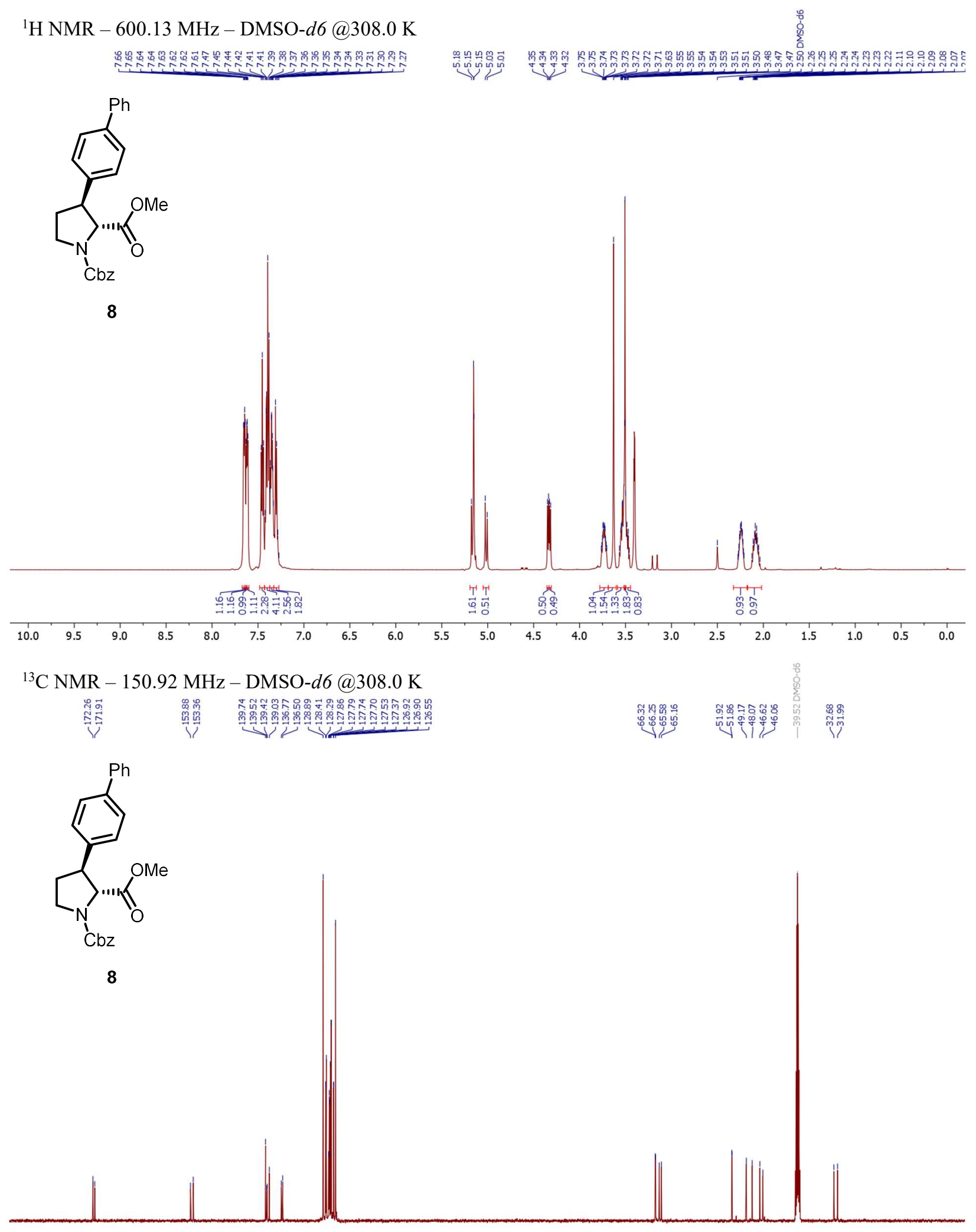

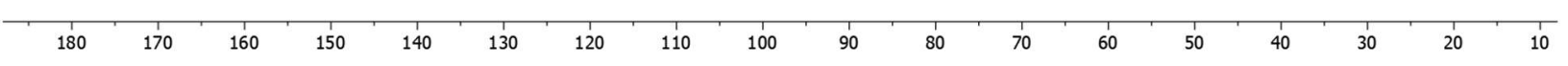




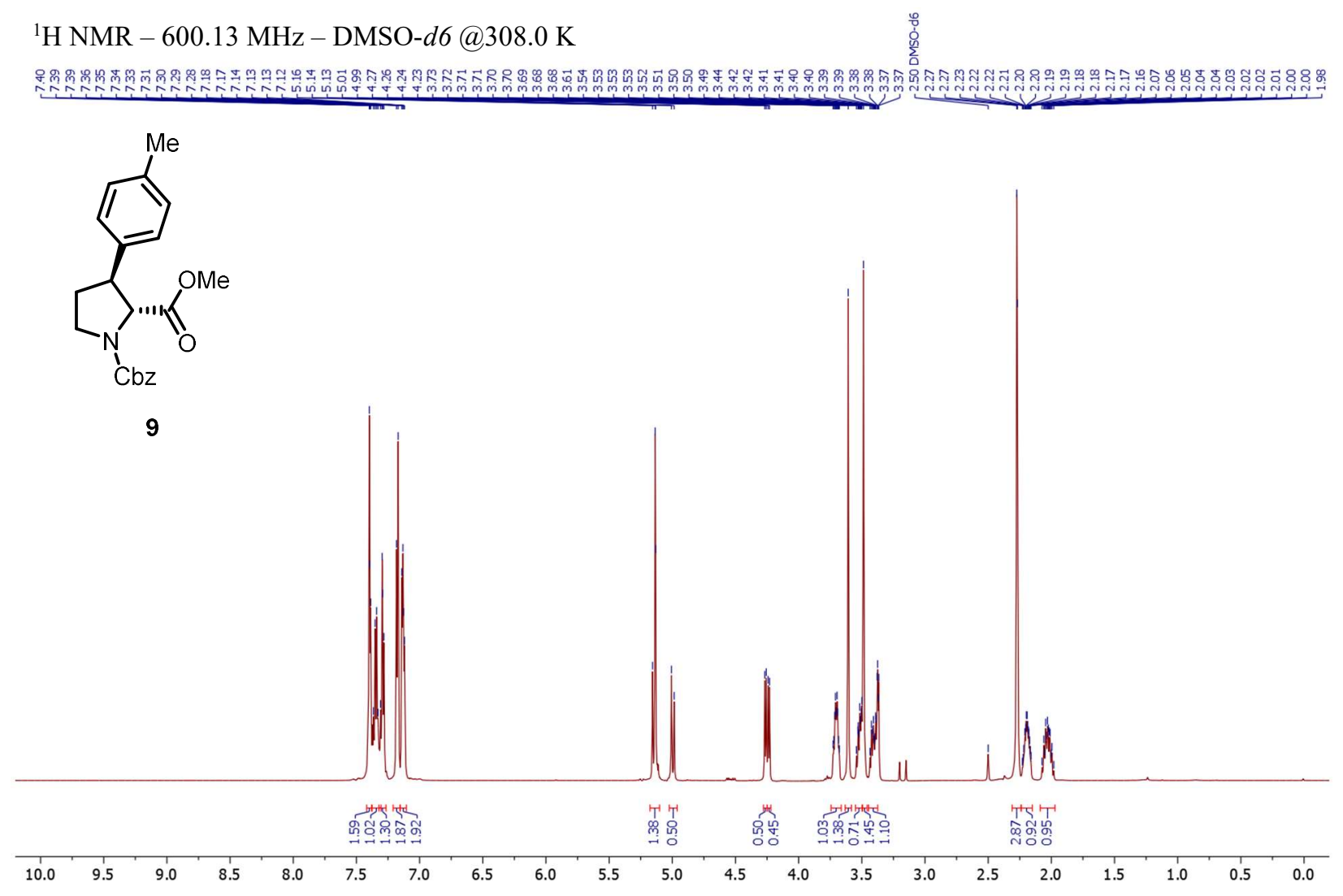

${ }^{13} \mathrm{C}$ NMR - 150.92 MHz - DMSO-d6@308.0 K

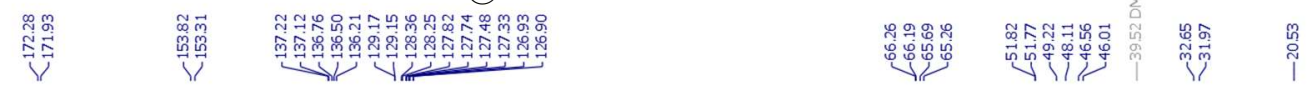<smiles>COC(=O)[C@H]1C(c2ccc(C)cc2)CCN1C(=O)[O-]</smiles>

9

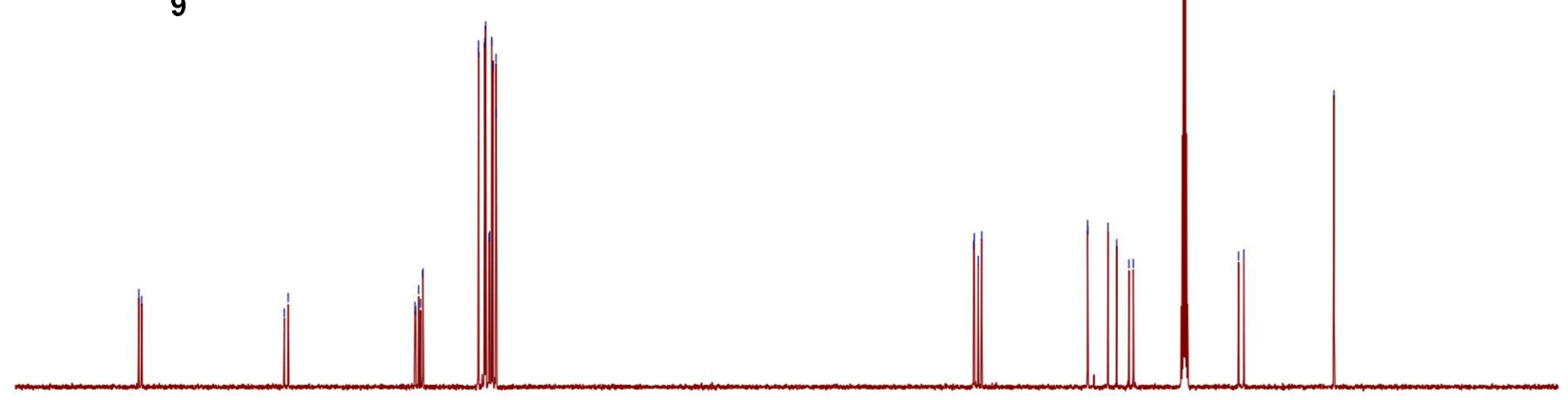

180

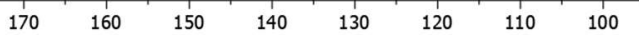

$90+80$


${ }^{1} \mathrm{H}$ NMR - 600.13 MHz - DMSO-d6@308.0 K

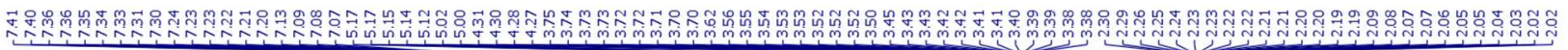<smiles>COC(=O)[C@H]1C(c2cccc(C)c2)CCN1C(=O)[O-]</smiles>

10

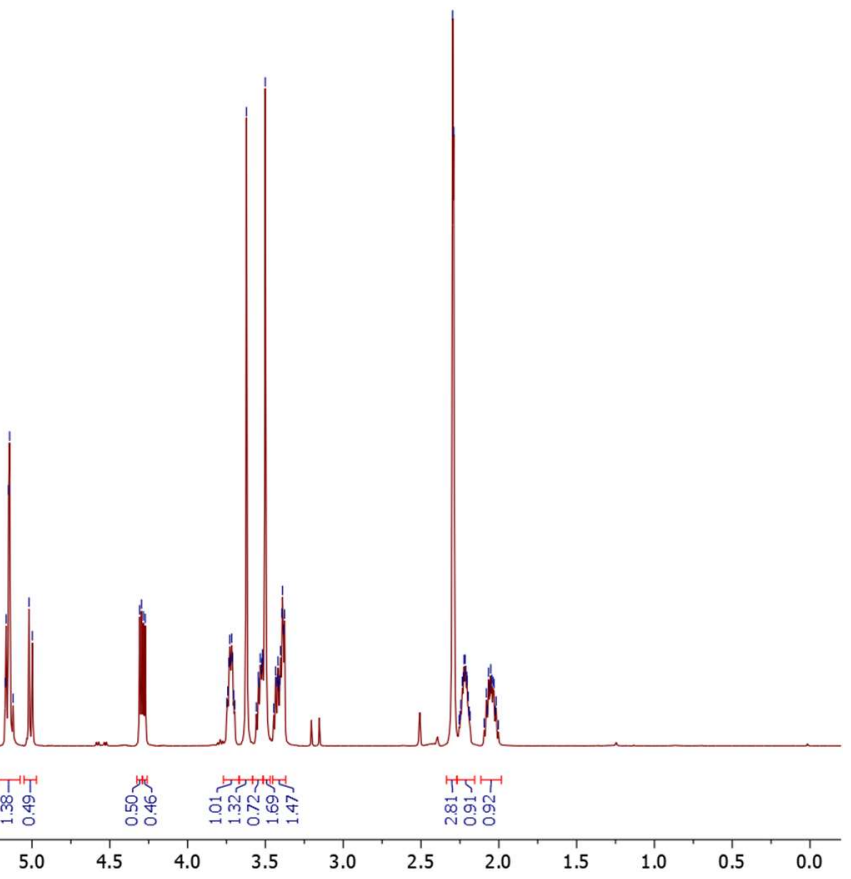

${ }^{13} \mathrm{C} \mathrm{NMR}$ - 150.92 MHz - DMSO-d6@308.0 K
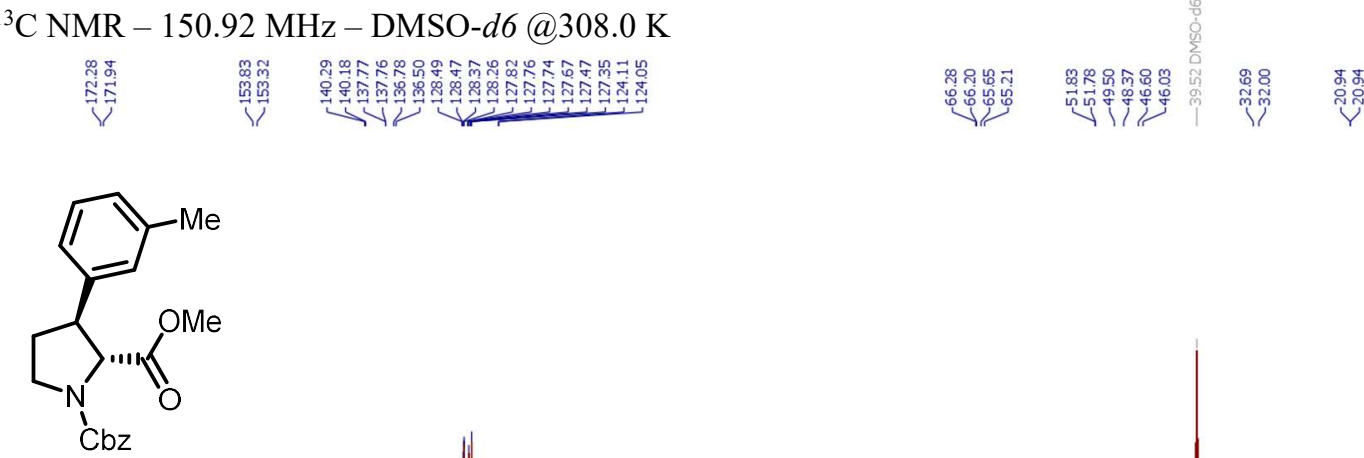

10

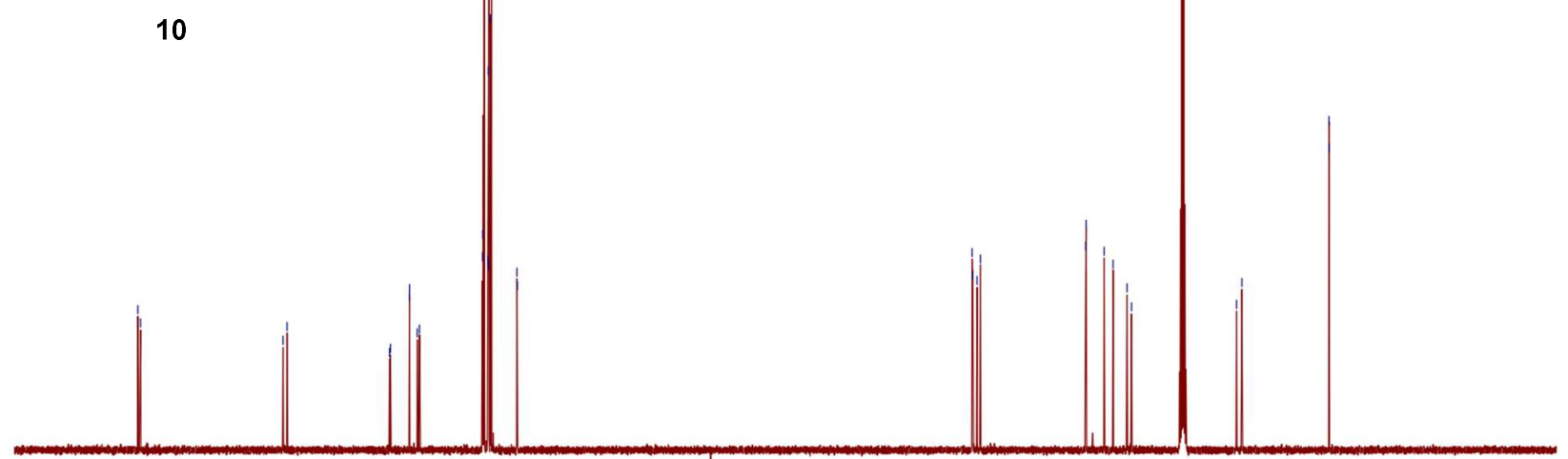

18

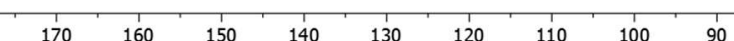




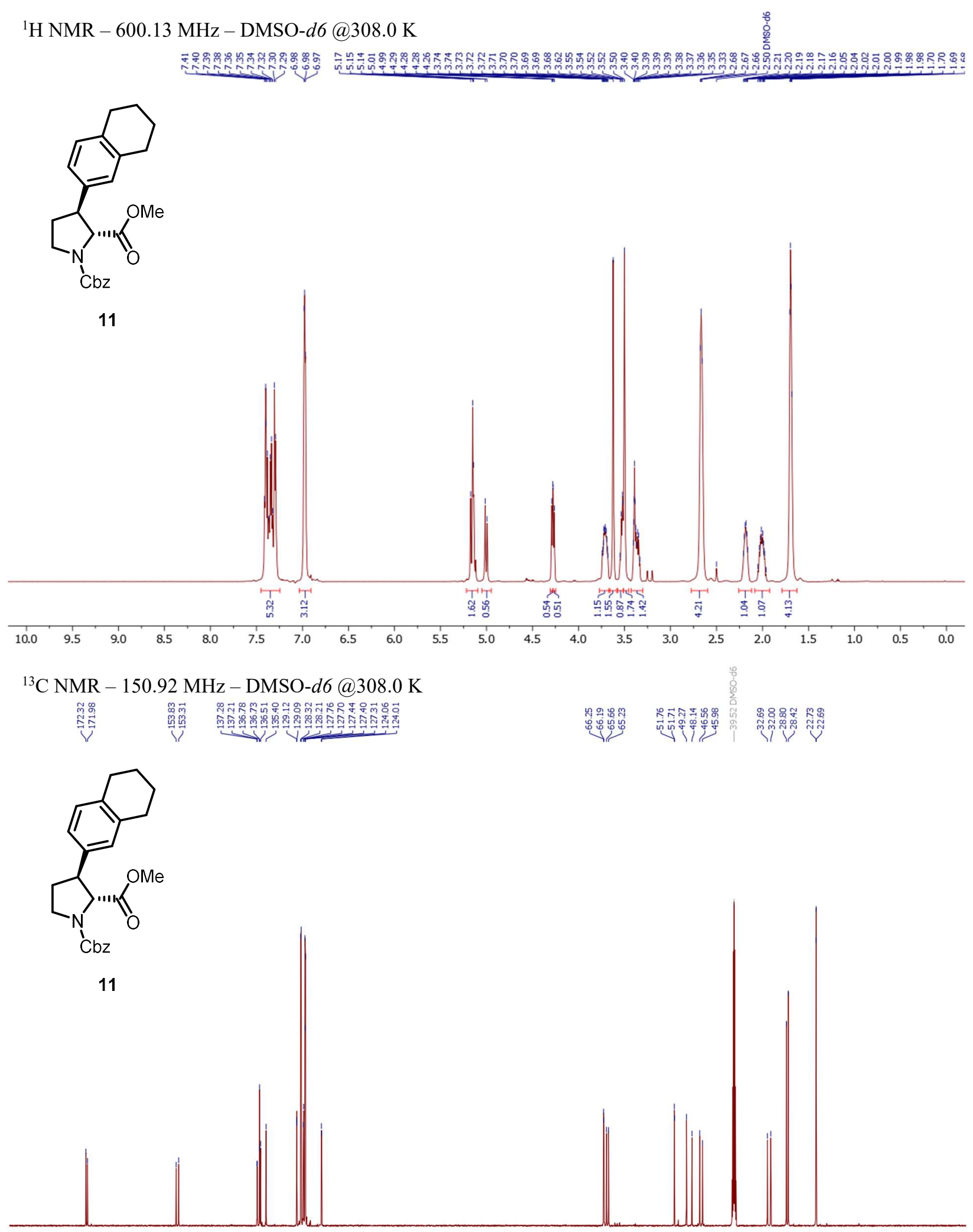

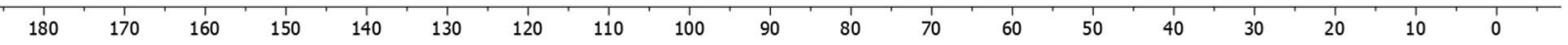



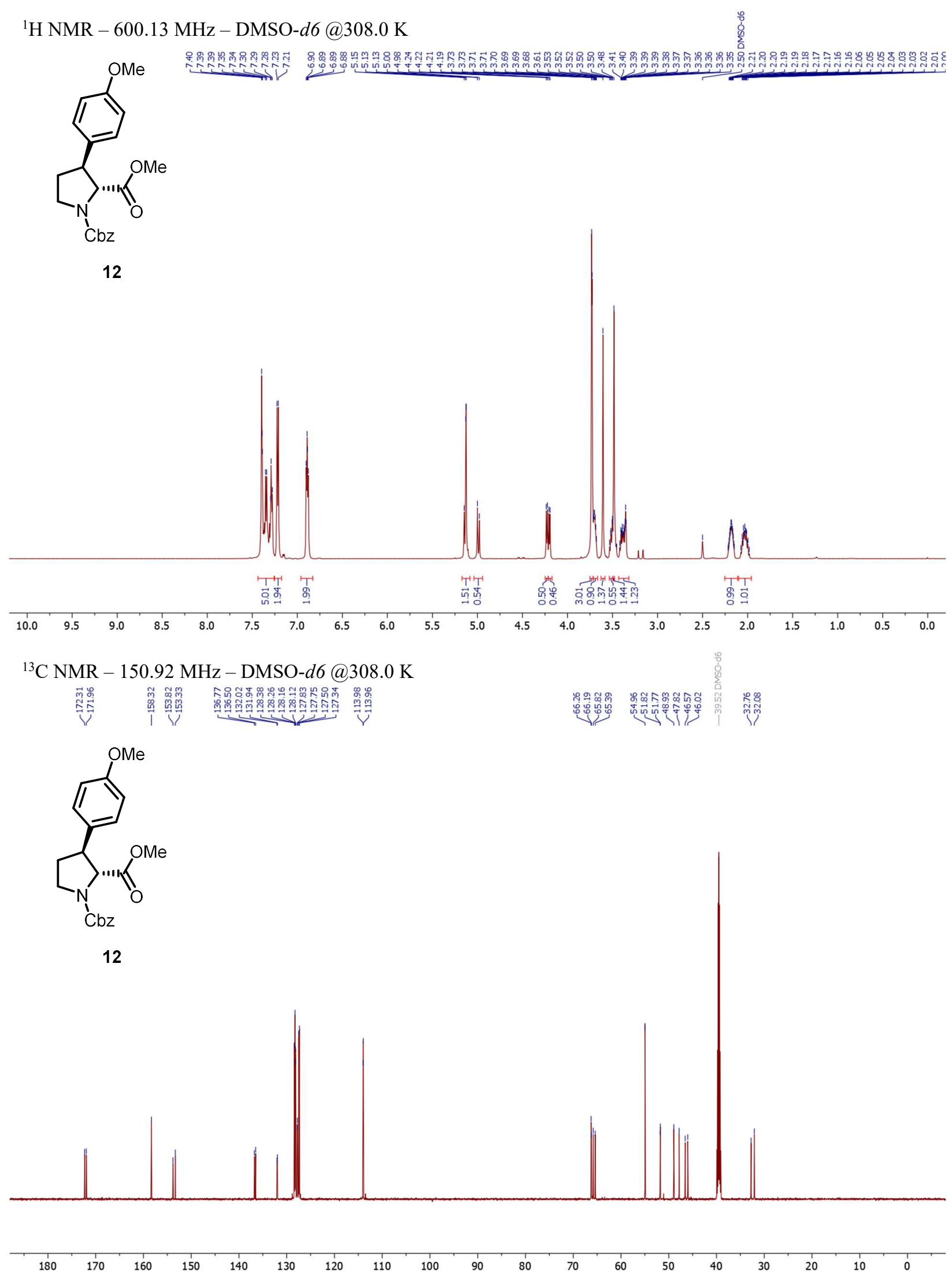
${ }^{1} \mathrm{H}$ NMR - 600.13 MHz-DMSO-d6@308.0 K
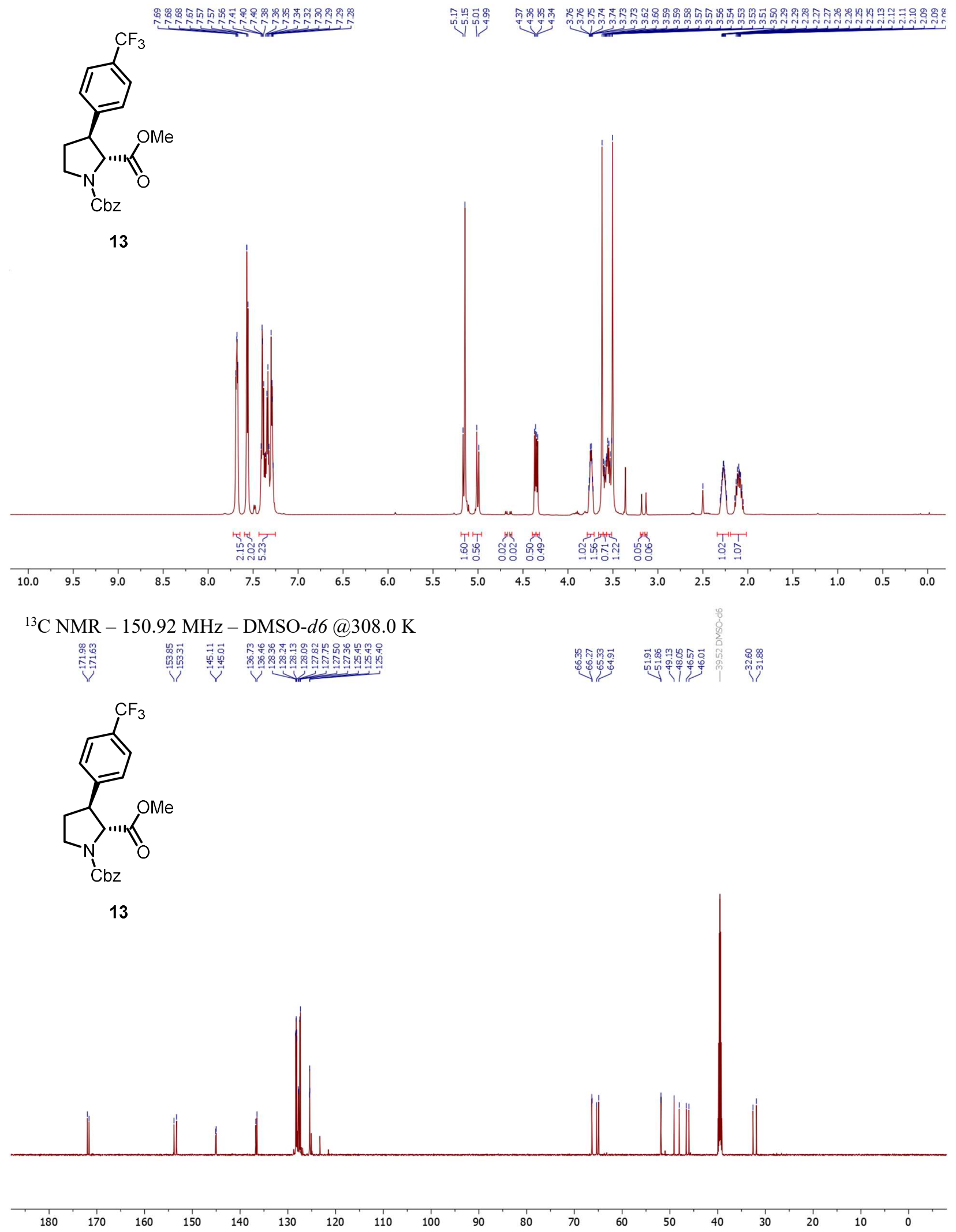


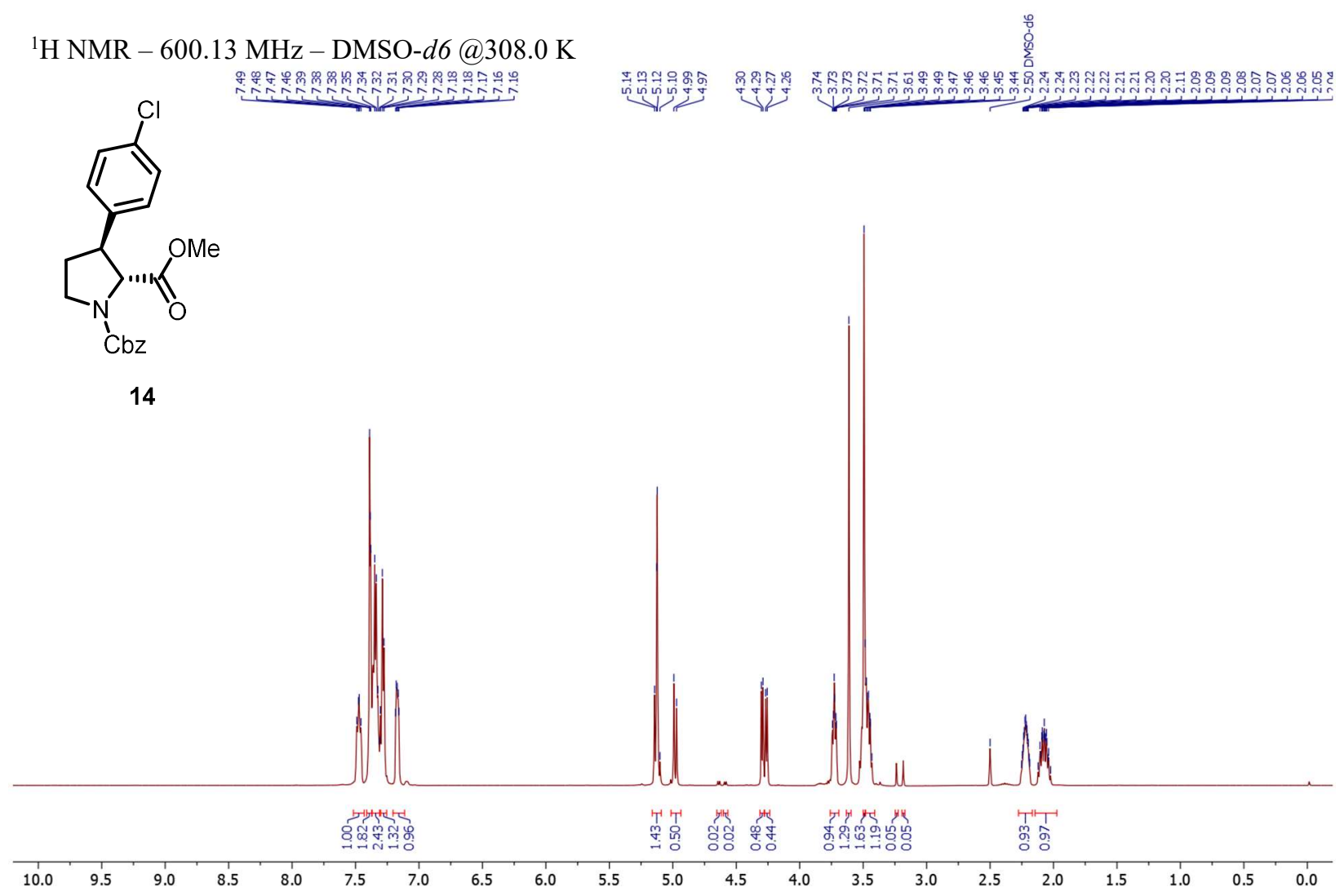

${ }^{13} \mathrm{C}$ NMR - $150.92 \mathrm{MHz}$ - DMSO-d6@308.0 K

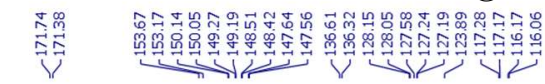<smiles>COC(=O)[C@@H]1CCN(C(=O)[O-])C1c1ccc(Cl)cc1</smiles>

14

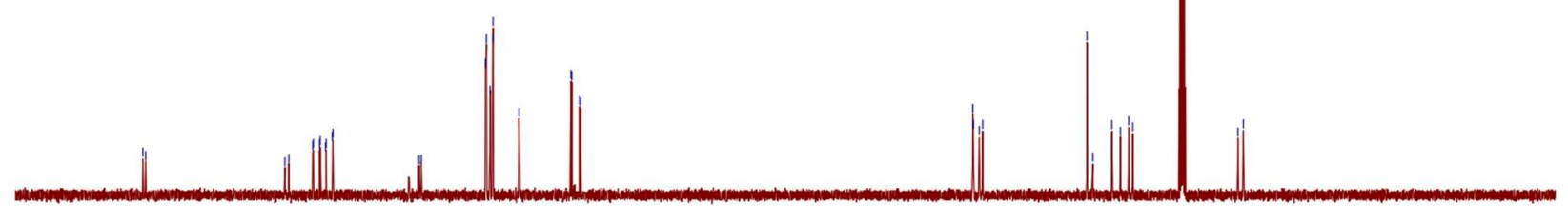

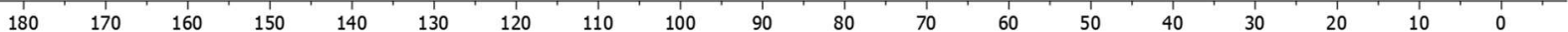


${ }^{1} \mathrm{H}$ NMR - 600.13 MHz - DMSO-d6@308.0 K

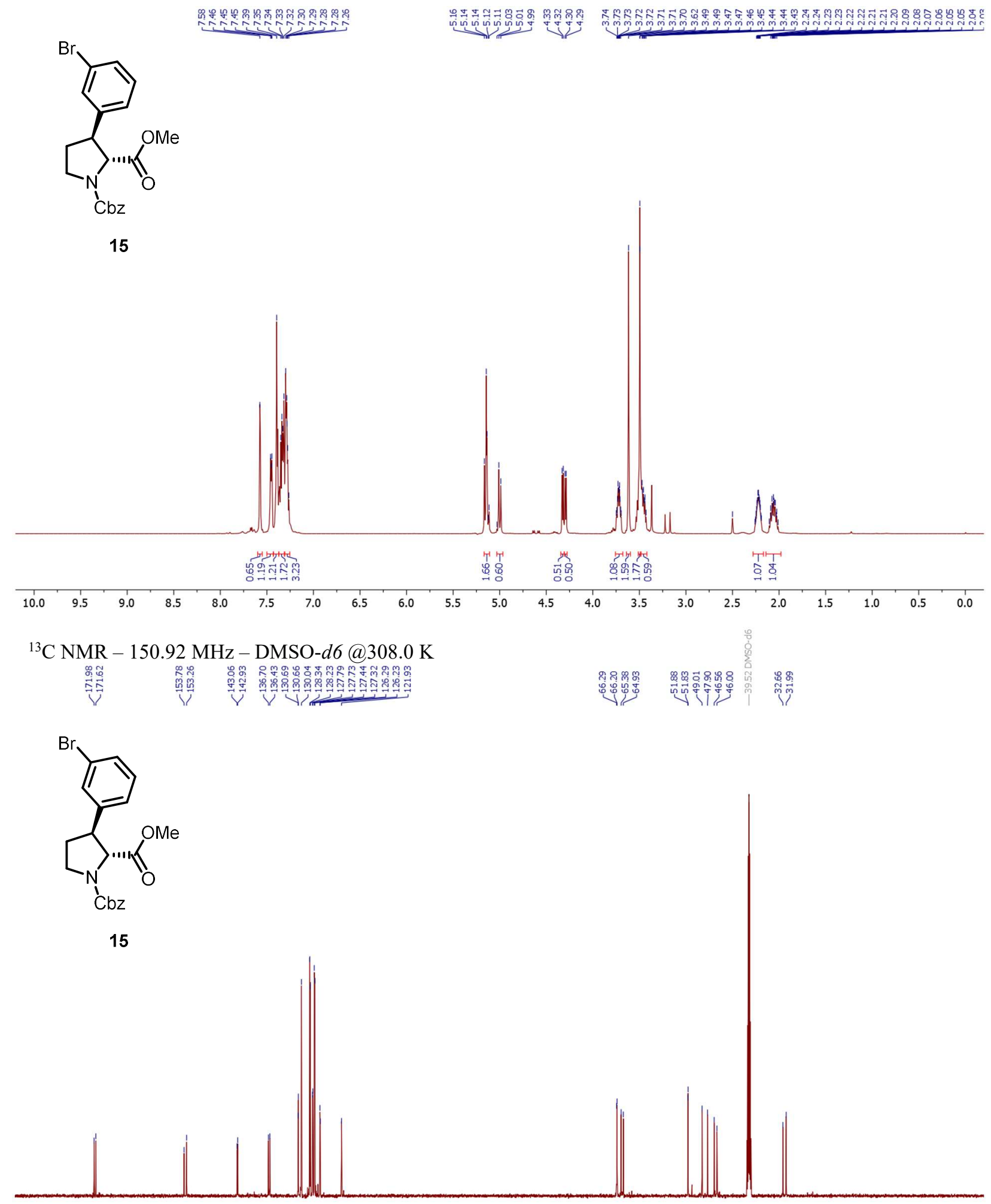

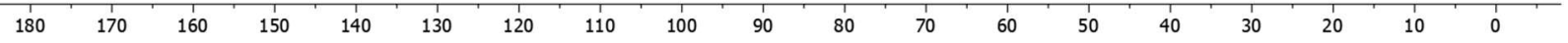


${ }^{1} \mathrm{H}$ NMR - 600.13 MHz-DMSO-d6@308.0 K

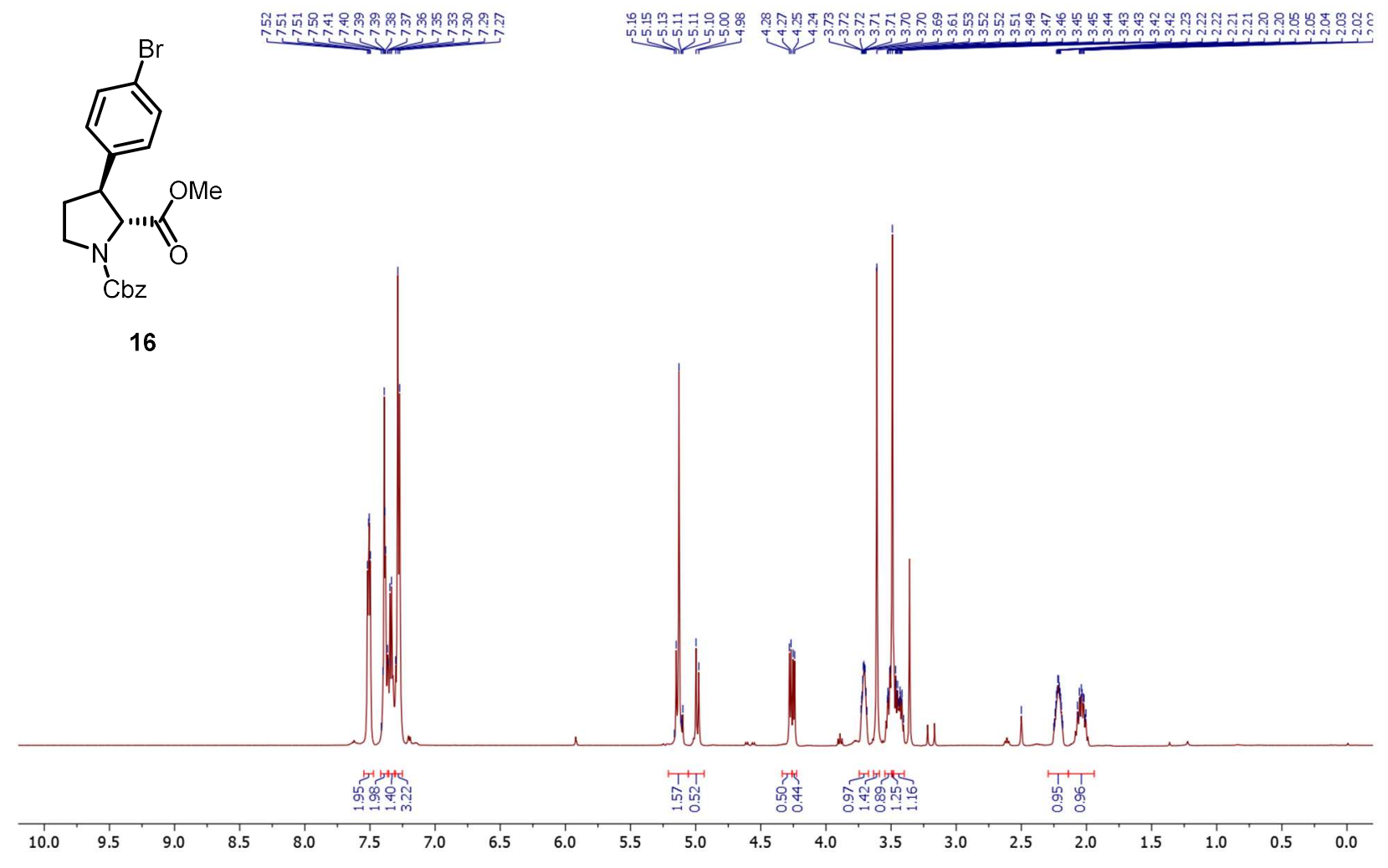

${ }^{13} \mathrm{C}$ NMR - 150.92 MHz - DMSO-d6@308.0 K

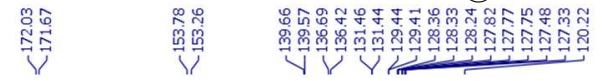

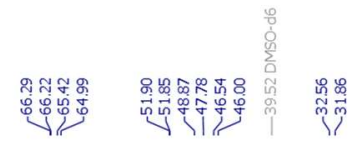<smiles>COC(=O)[C@H]1[C@@H](c2ccc(Br)cc2)CCN1C(=O)OCc1ccccc1</smiles>

16
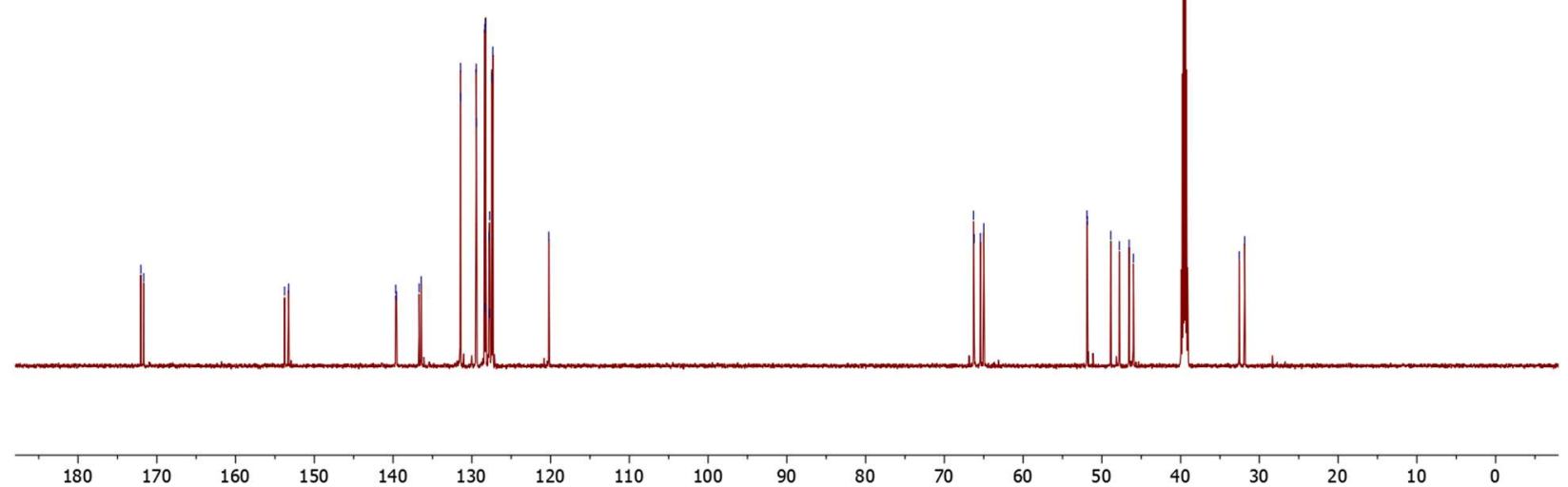

$90 \quad 80$ 

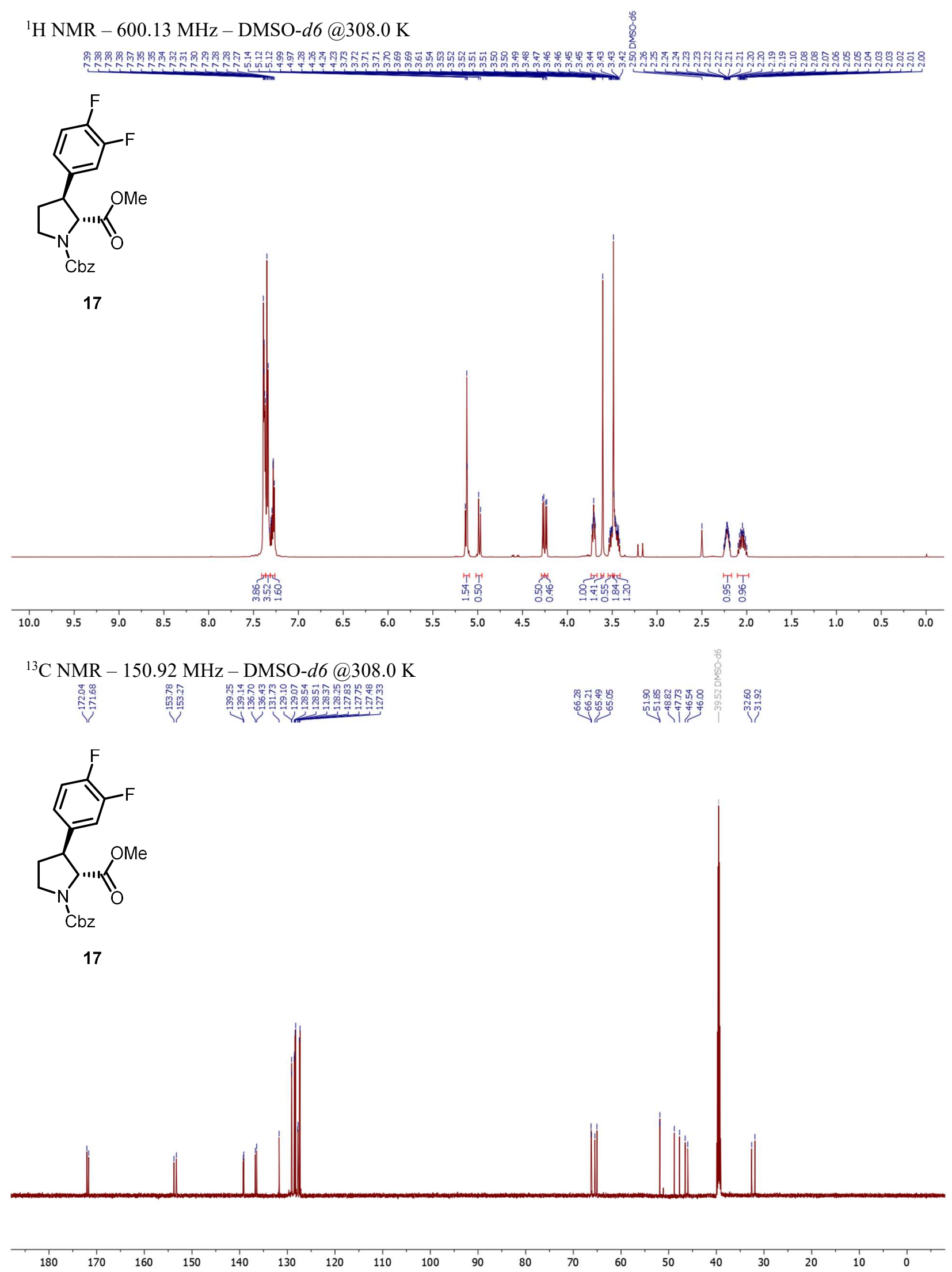

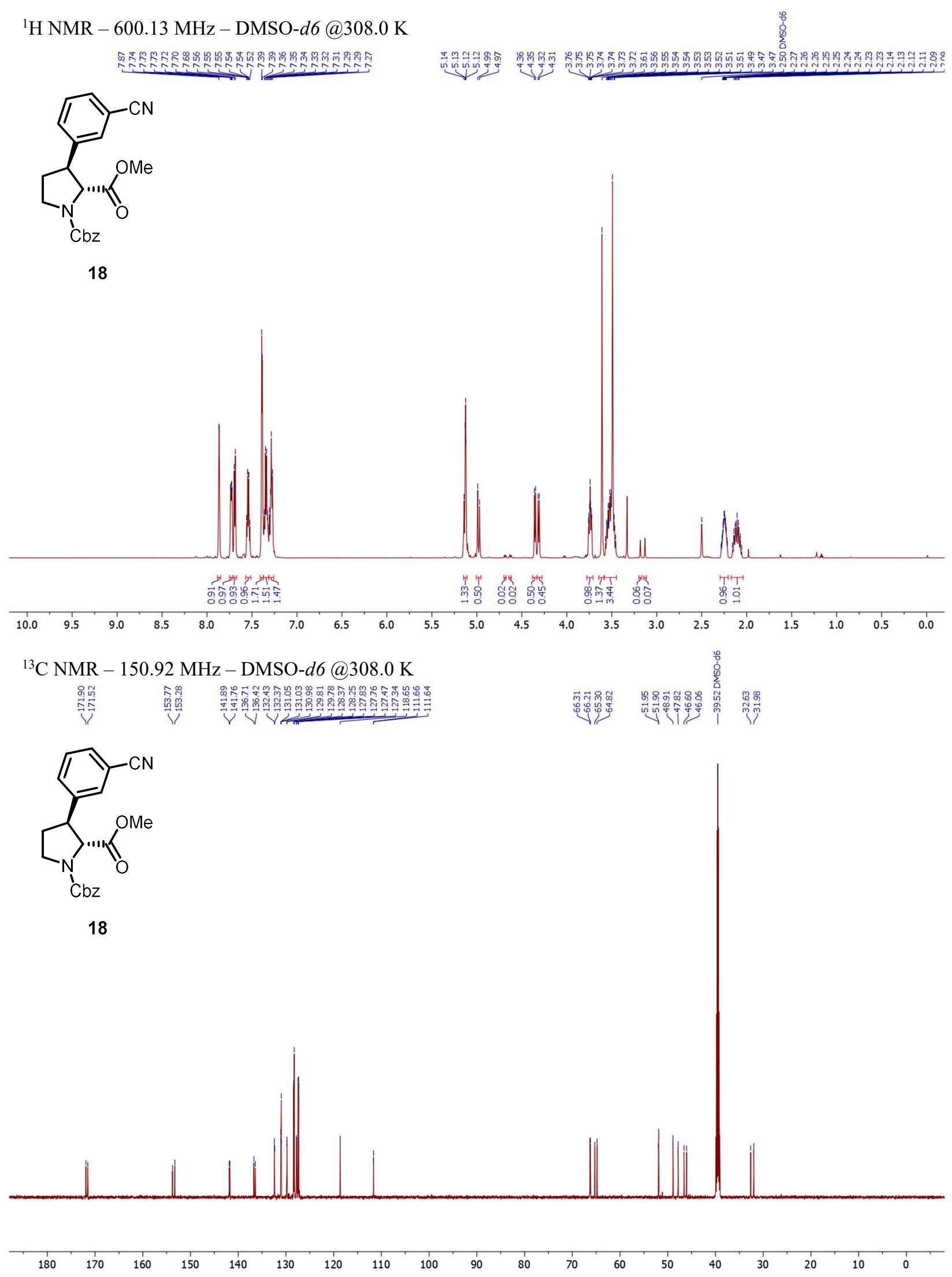

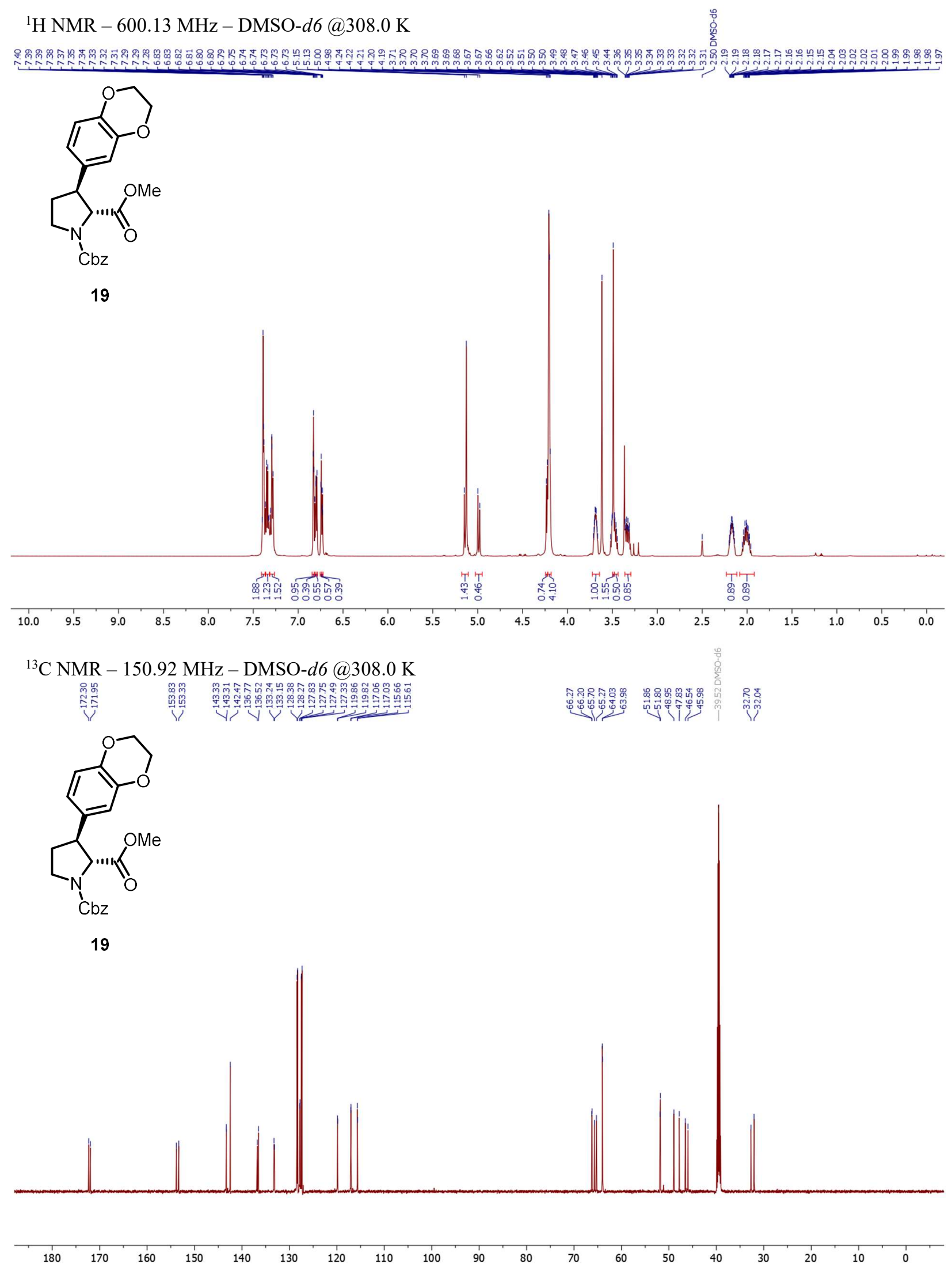


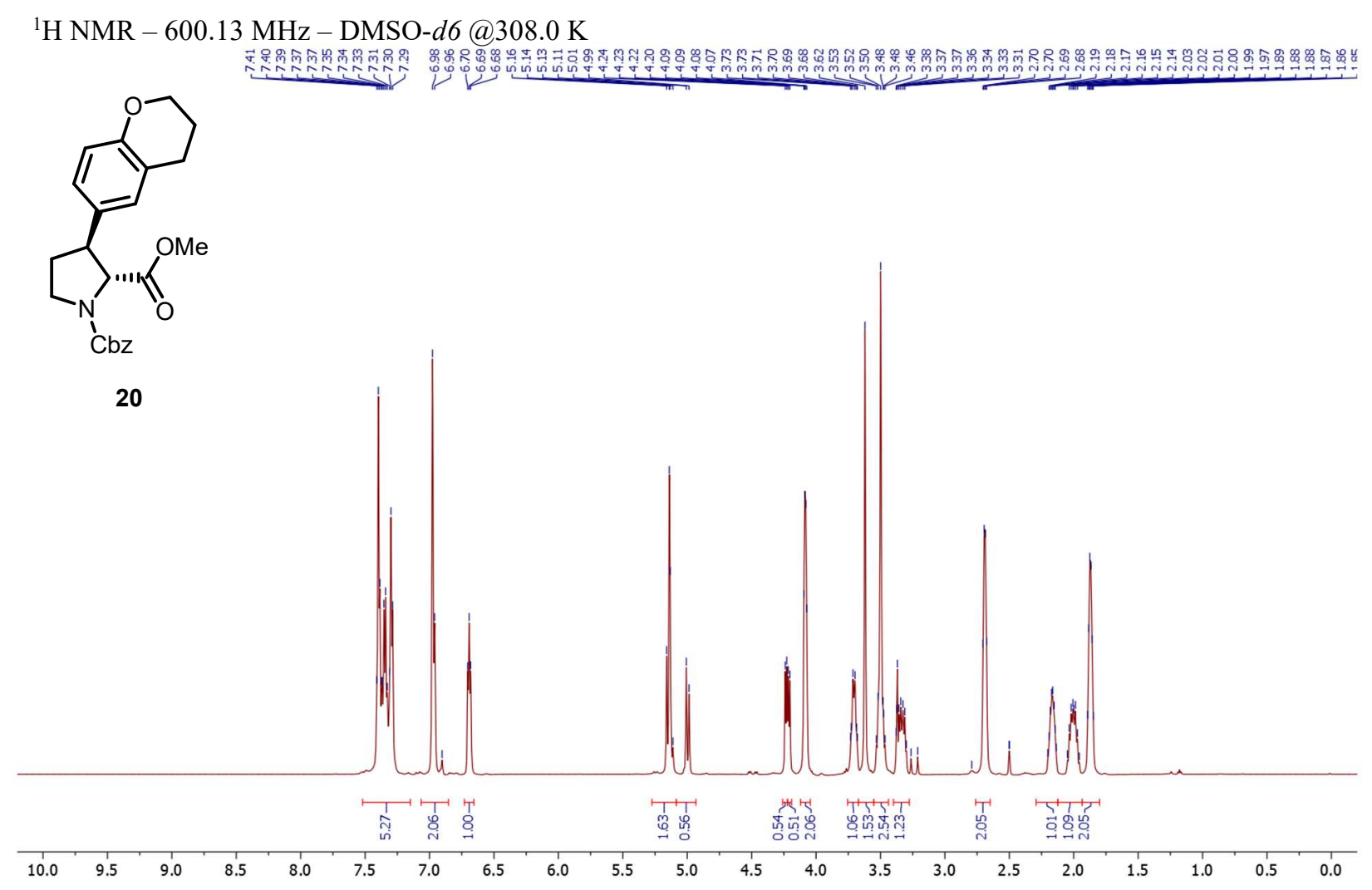

${ }^{13} \mathrm{C}$ NMR - $150.92 \mathrm{MHz}$ - DMSO-d6@308.0 K

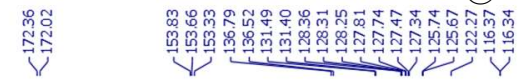
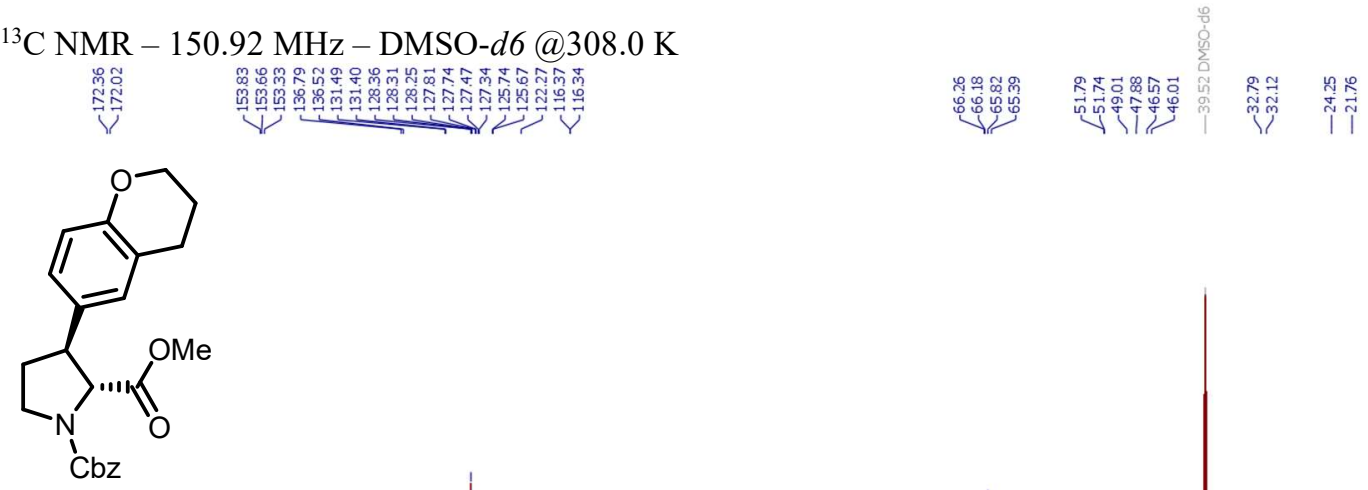

20

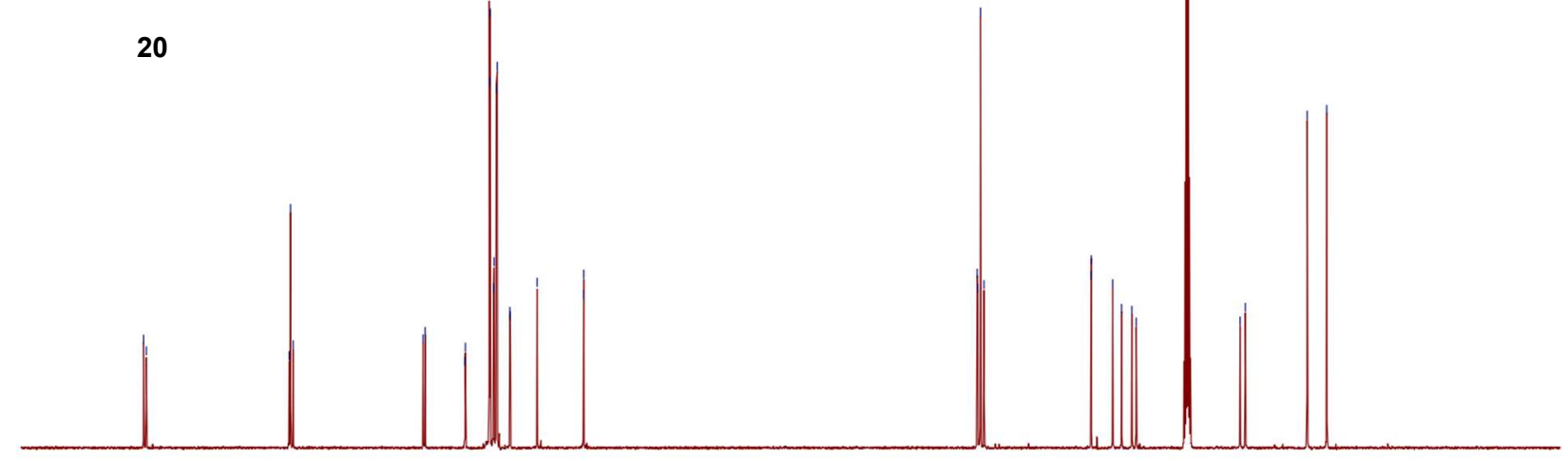


${ }^{1} \mathrm{H}$ NMR - 600.13 MHz - DMSO-d6@308.0 K<smiles>COC(=O)[C@H]1[C@@H](c2ccc3c(c2)OCO3)CCN1C(=O)OCc1ccccc1</smiles>

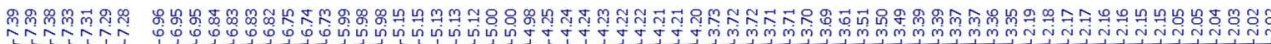

21

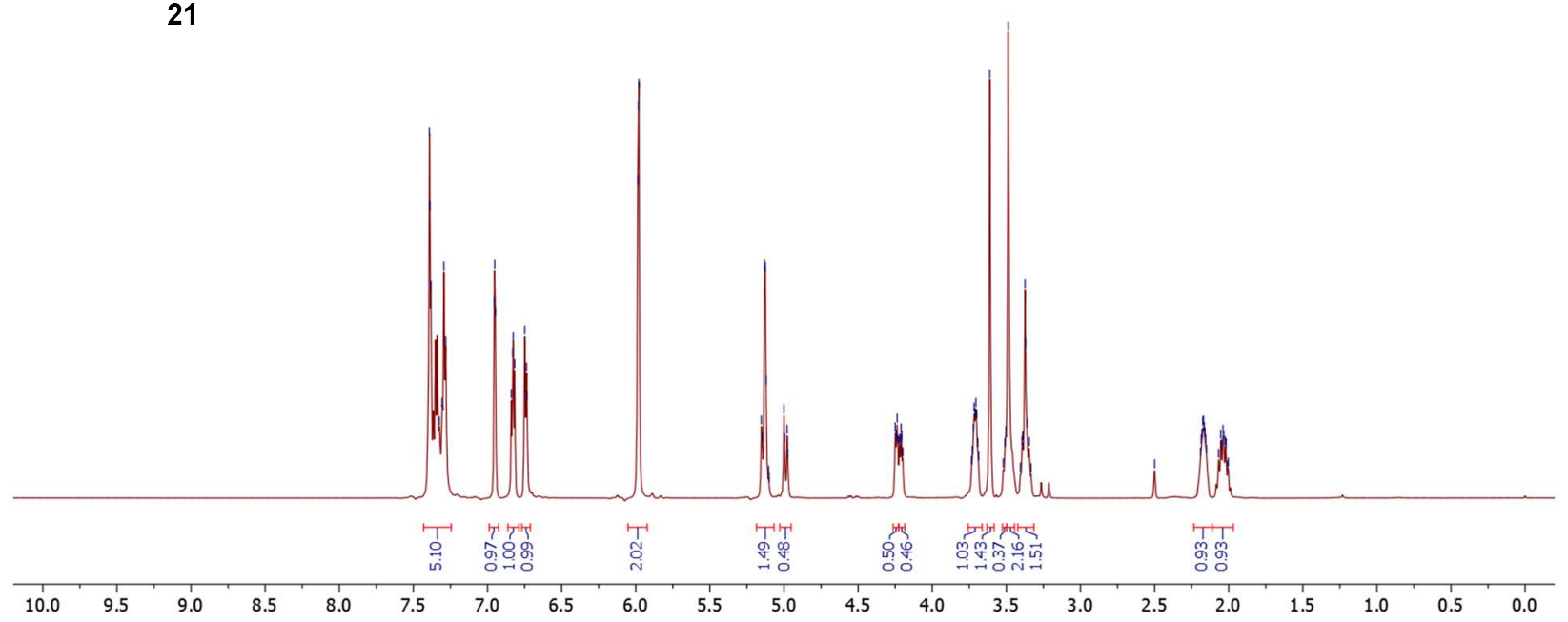

${ }^{13} \mathrm{C}$ NMR - $150.92 \mathrm{MHz}$ - DMSO-d6@308.0 K

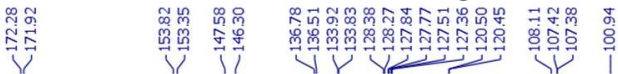
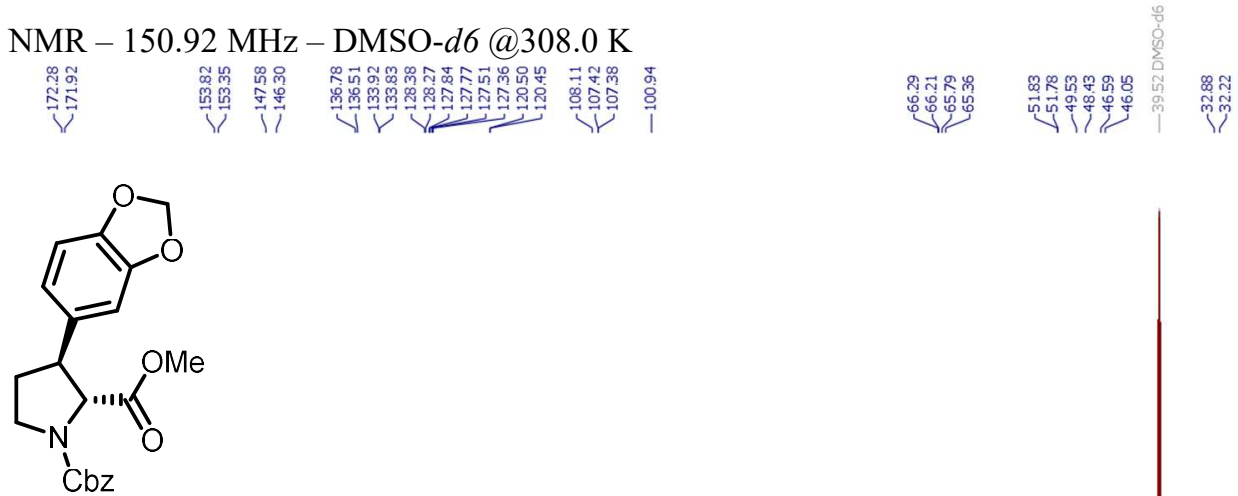

21

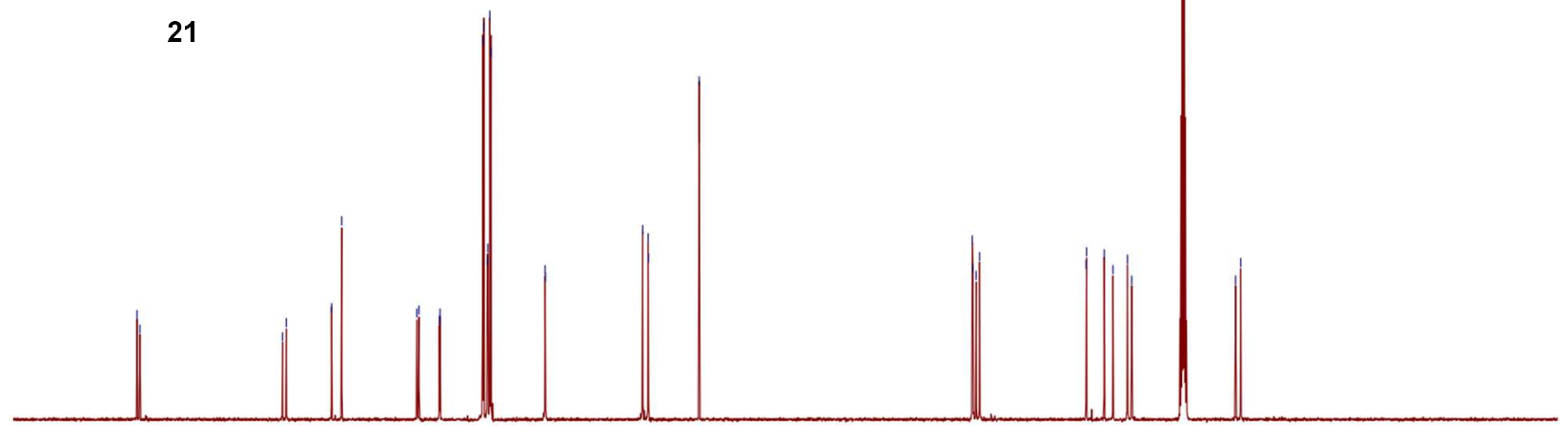

180

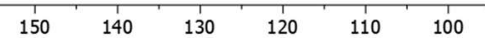

90 
${ }^{1} \mathrm{H}$ NMR - 600.13 MHz - DMSO-d6@308.0 K

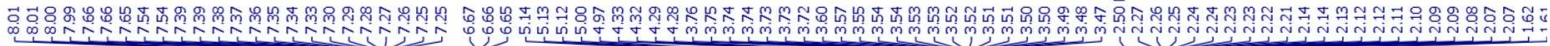<smiles>COC(=O)[C@@H]1[C@@H](c2ccc3c(ccn3C(=O)OC(C)(C)C)c2)CCN1C(=O)OCc1ccccc1</smiles>

22

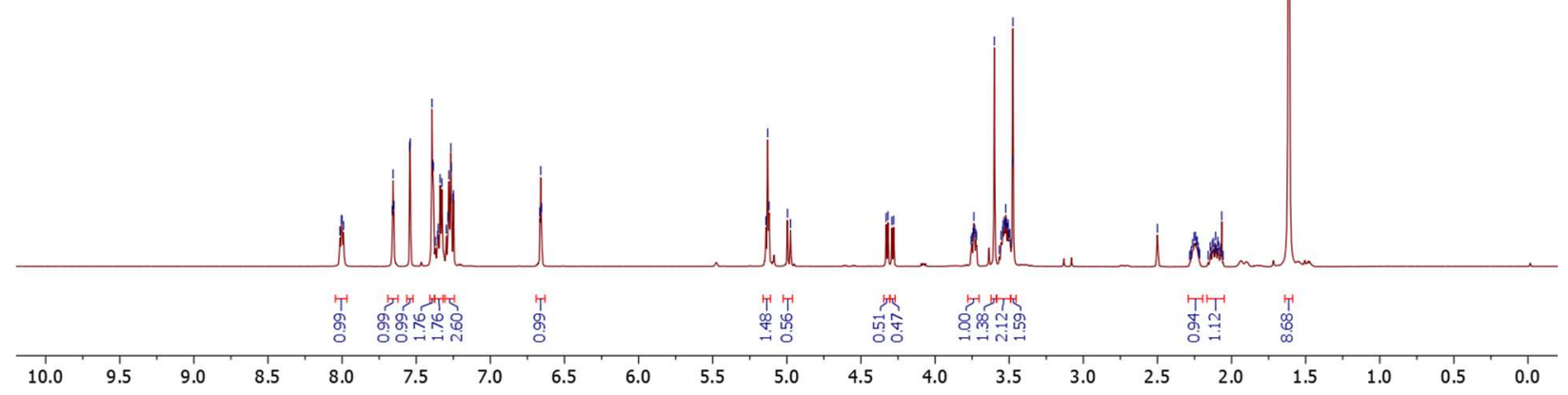

${ }^{13} \mathrm{C}$ NMR - 150.92 MHz - DMSO-d6@308.0 K 穿<smiles>COC(=O)[C@H]1[C@@H](c2ccc3c(ccn3C(=O)OC(C)(C)C)c2)CCN1C(=O)OCc1ccccc1</smiles>

22
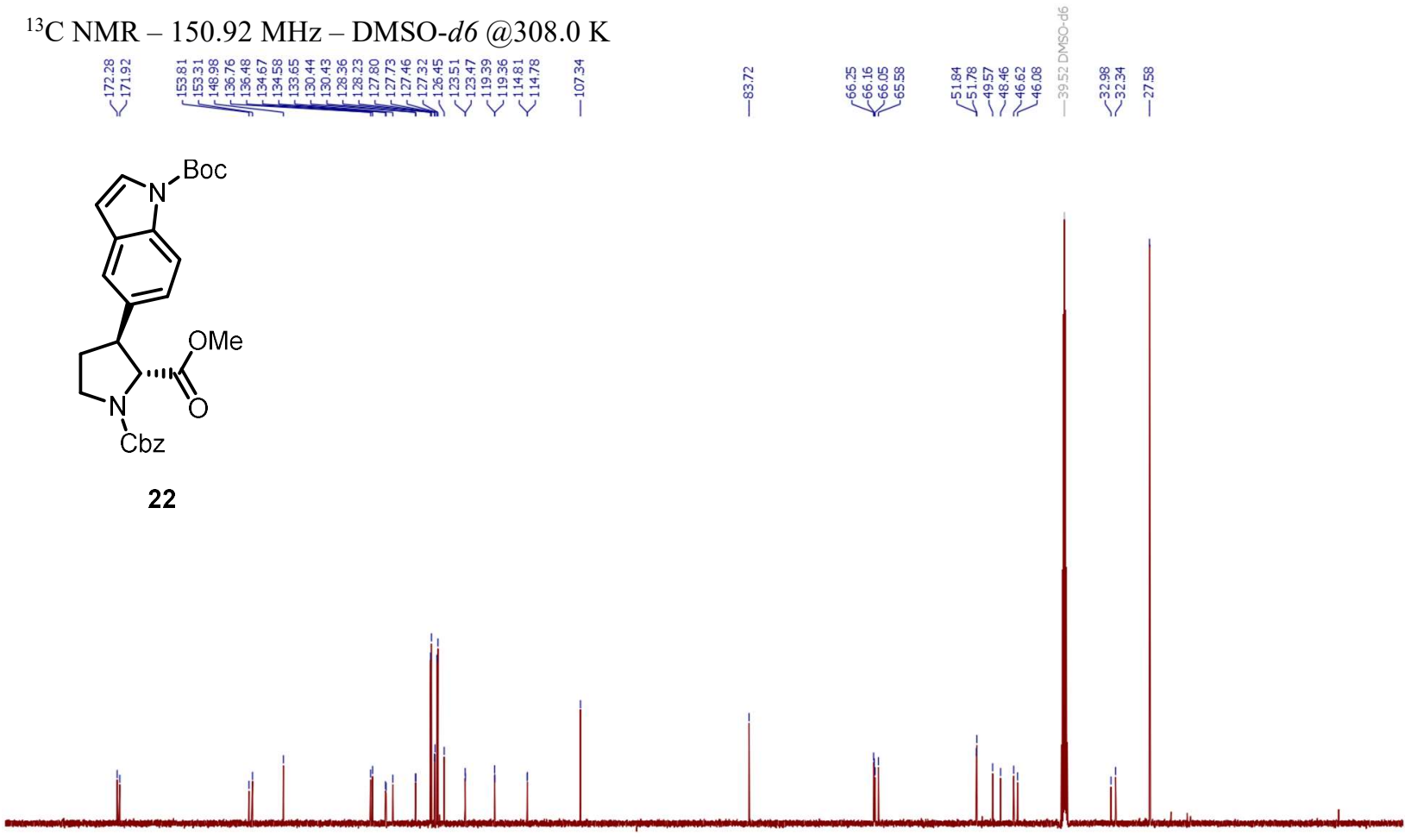

180 
${ }^{1} \mathrm{H}$ NMR - 600.13 MHz - DMSO-d6@308.0 K

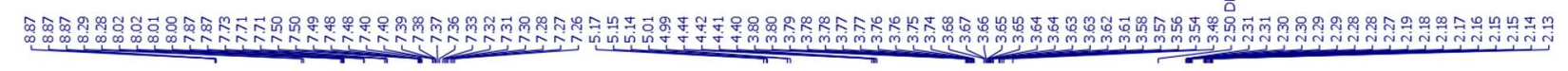<smiles>COC(=O)[C@H]1[C@@H](c2ccc3ncccc3c2)CCN1C(=O)[O-]</smiles>

23

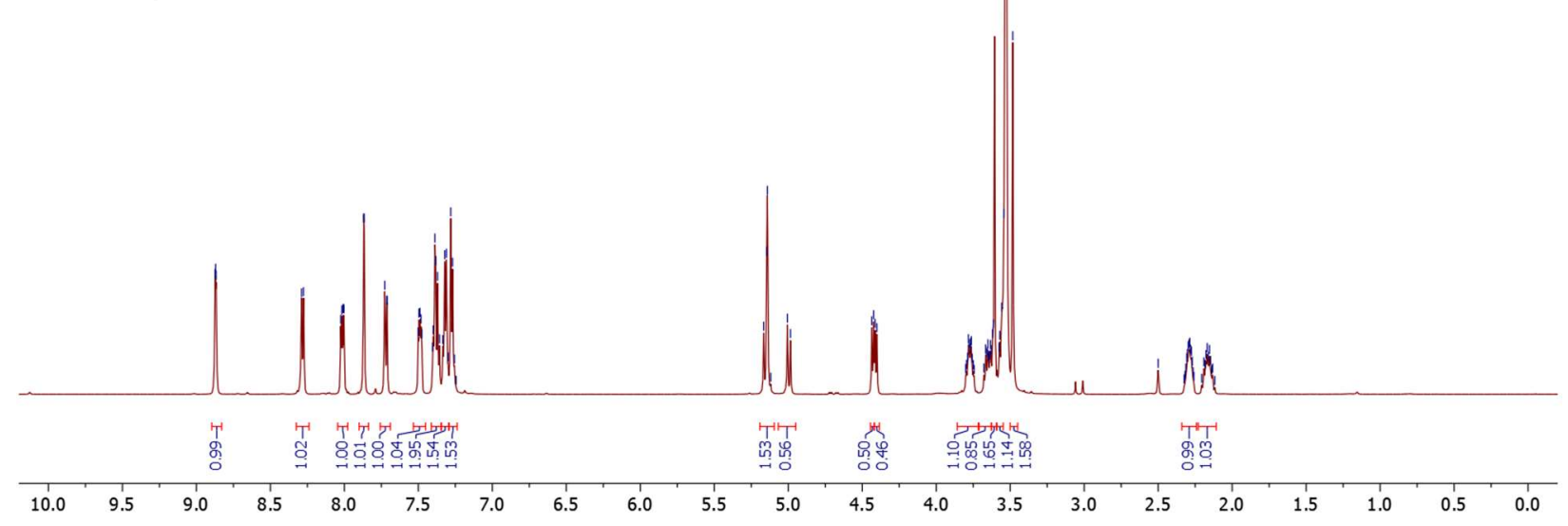

${ }^{13} \mathrm{C}$ NMR - $150.92 \mathrm{MHz}$ - DMSO-d6@308.0 K

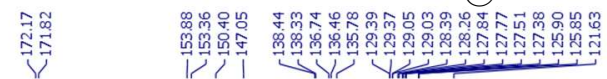<smiles>COC(=O)[C@H]1[C@@H](c2ccc3ncccc3c2)CCN1C(=O)[O-]</smiles>

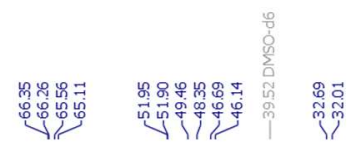

23

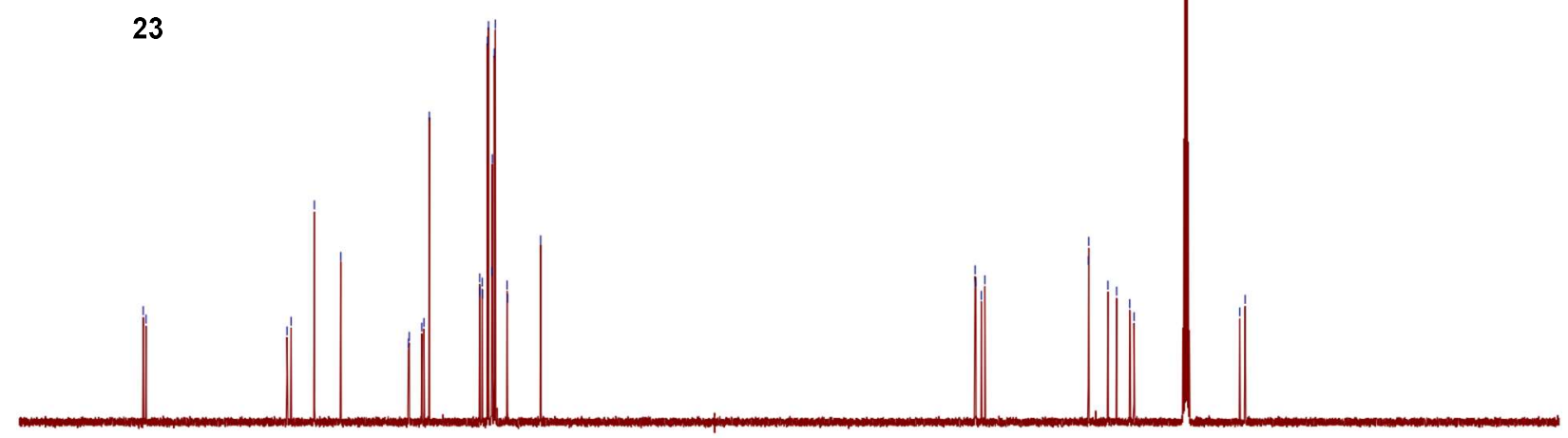


${ }^{1} \mathrm{H}$ NMR - 600.13 MHz - DMSO-d6@308.0 K

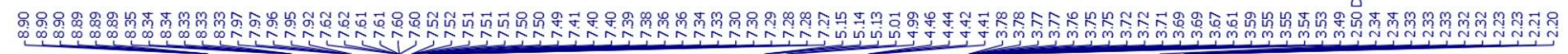<smiles>COC(=O)[C@H]1[C@@H](c2ccc3cccnc3c2)CCN1C(=O)[O-]</smiles>

24

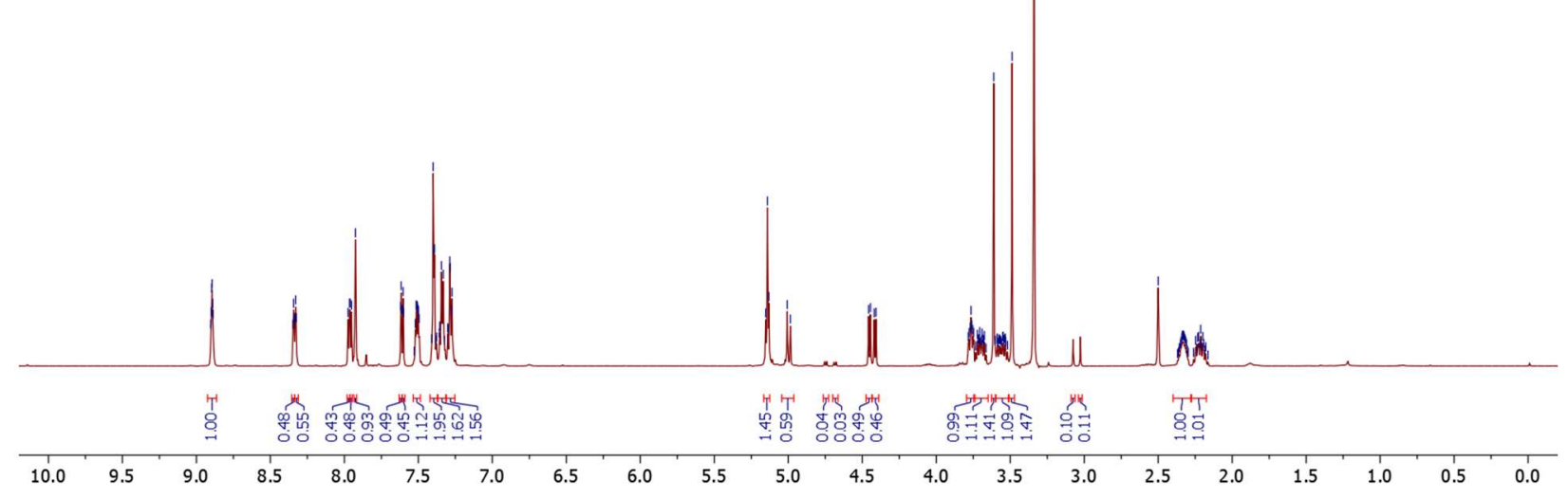

${ }^{13} \mathrm{C}$ NMR - $150.92 \mathrm{MHz}$ - DMSO-d6@308.0 K

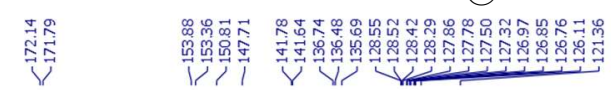<smiles>COC(=O)[C@@H]1[C@@H](c2ccc3cccnc3c2)CCN1C(=O)[O-]</smiles>

24

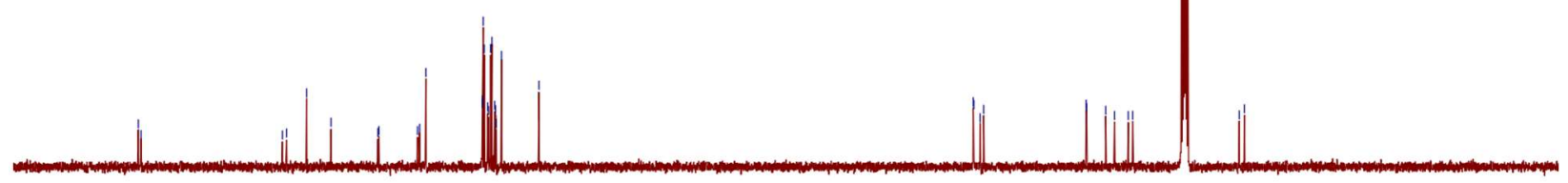

$180-170 \quad 160-150,140$
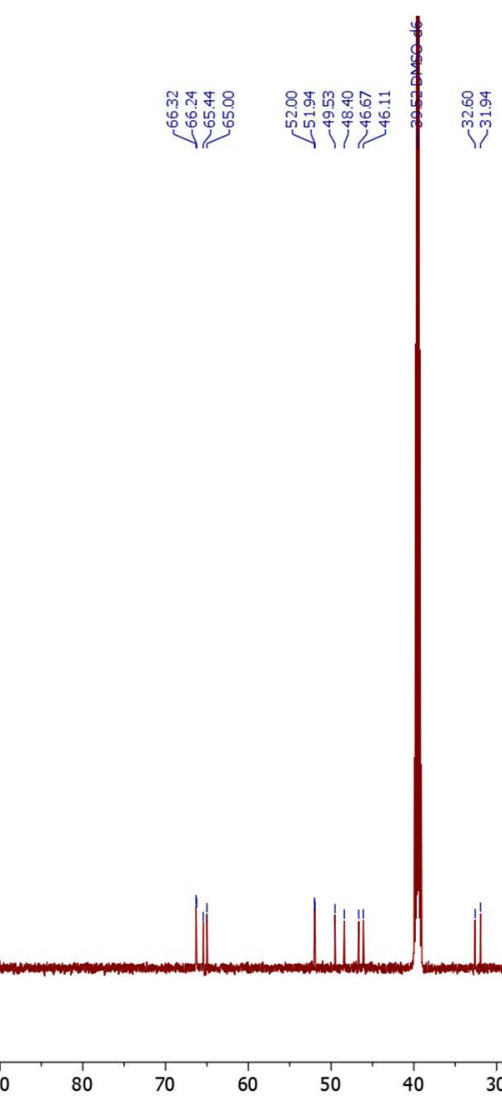

180

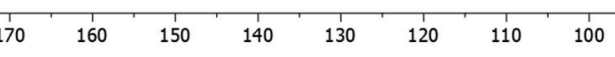



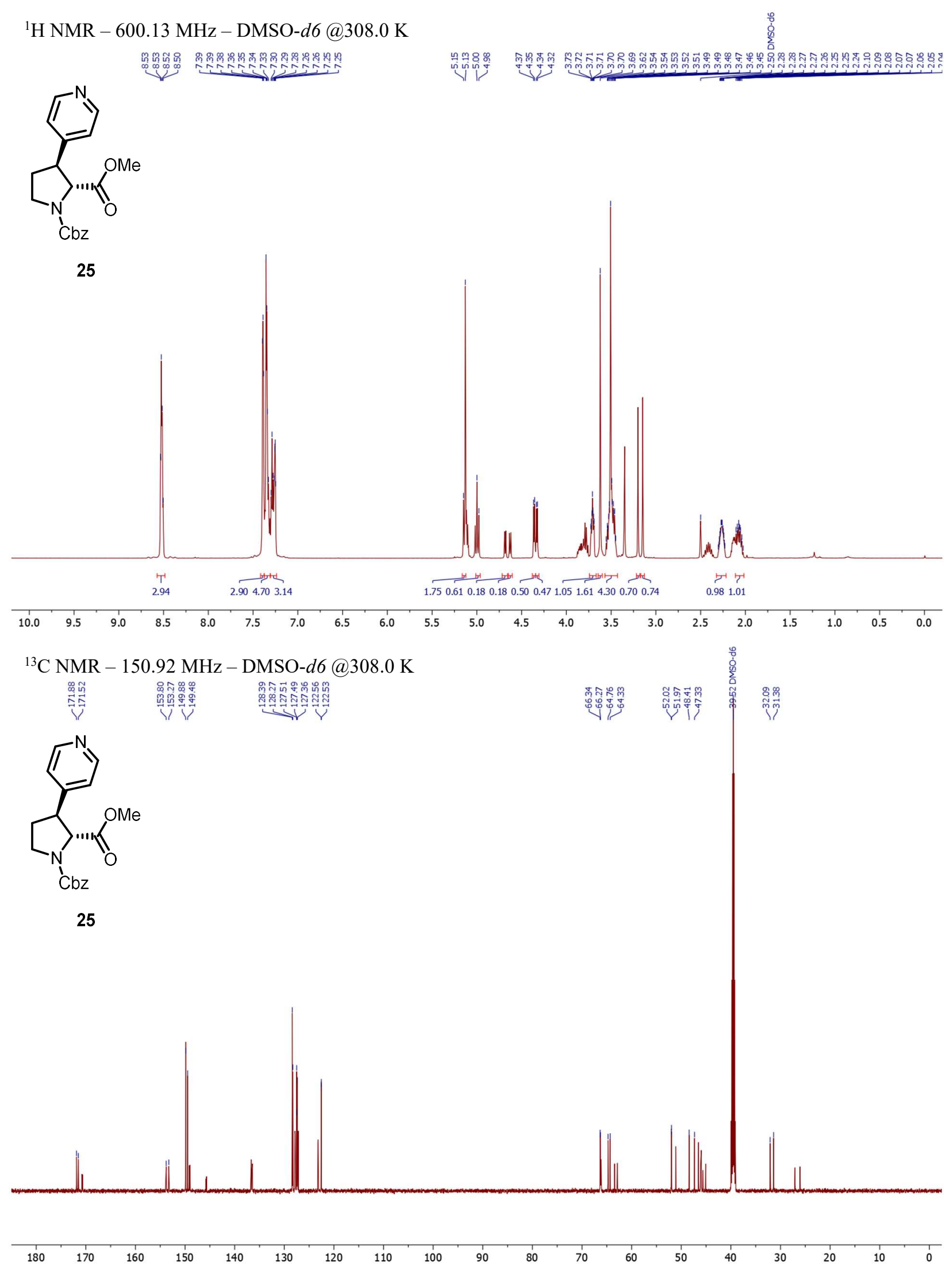


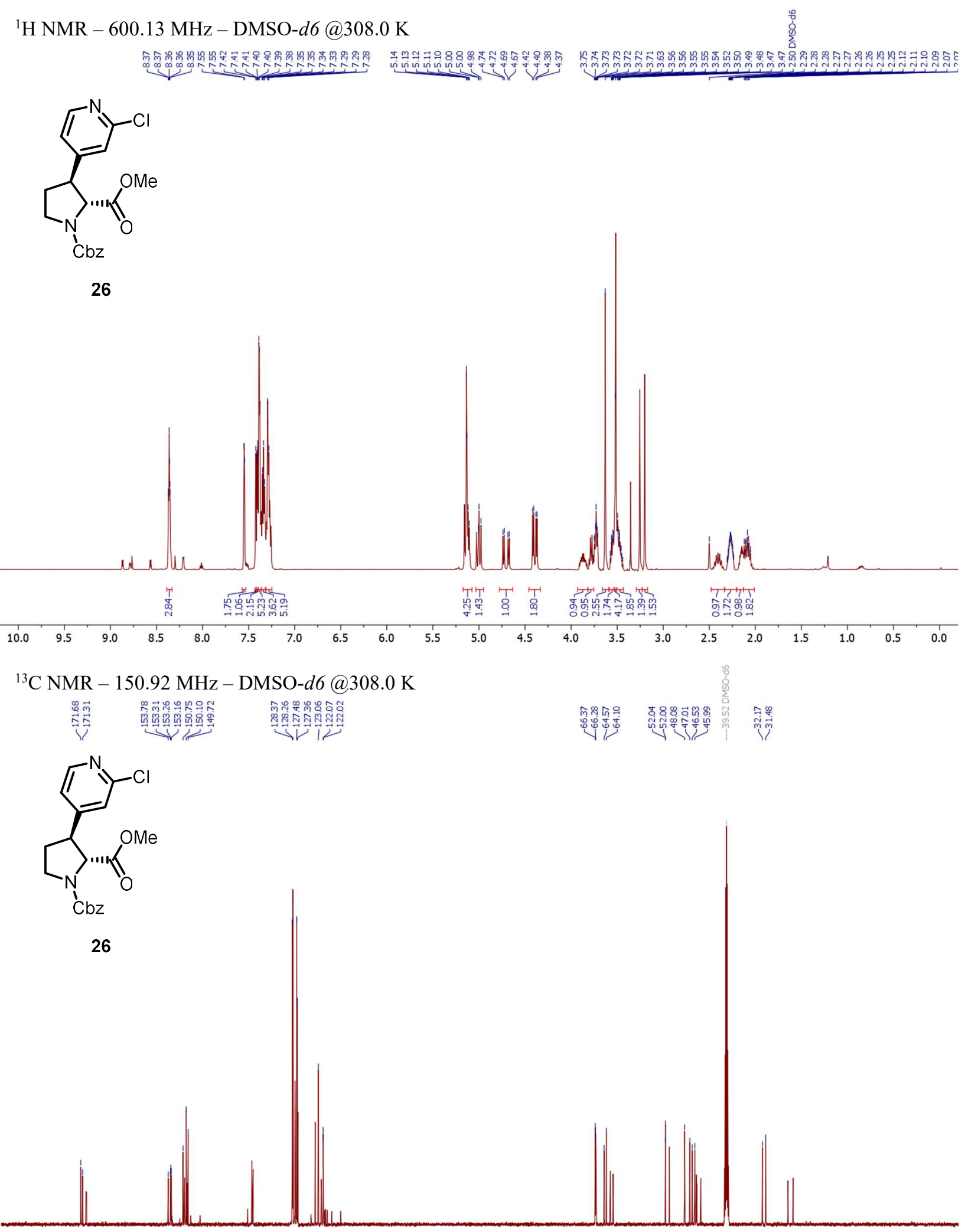

$\begin{array}{lllllllllllllllllll}180 & 170 & 160 & 150 & 140 & 130 & 120 & 110 & 100 & 90 & 80 & 70 & 60 & 50 & 40 & 30 & 20 & 10 & 0\end{array}$ 
${ }^{1} \mathrm{H}$ NMR - 600.13 MHz - DMSO-d6@308.0 K

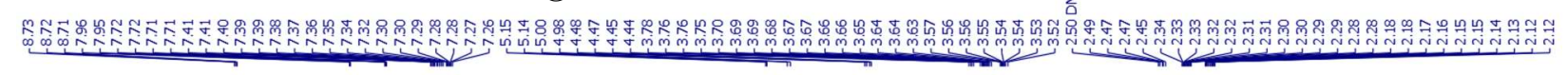<smiles>COC(=O)[C@H]1[C@@H](c2ccnc(C(F)(F)F)c2)CCN1C(=O)[O-]</smiles>

27

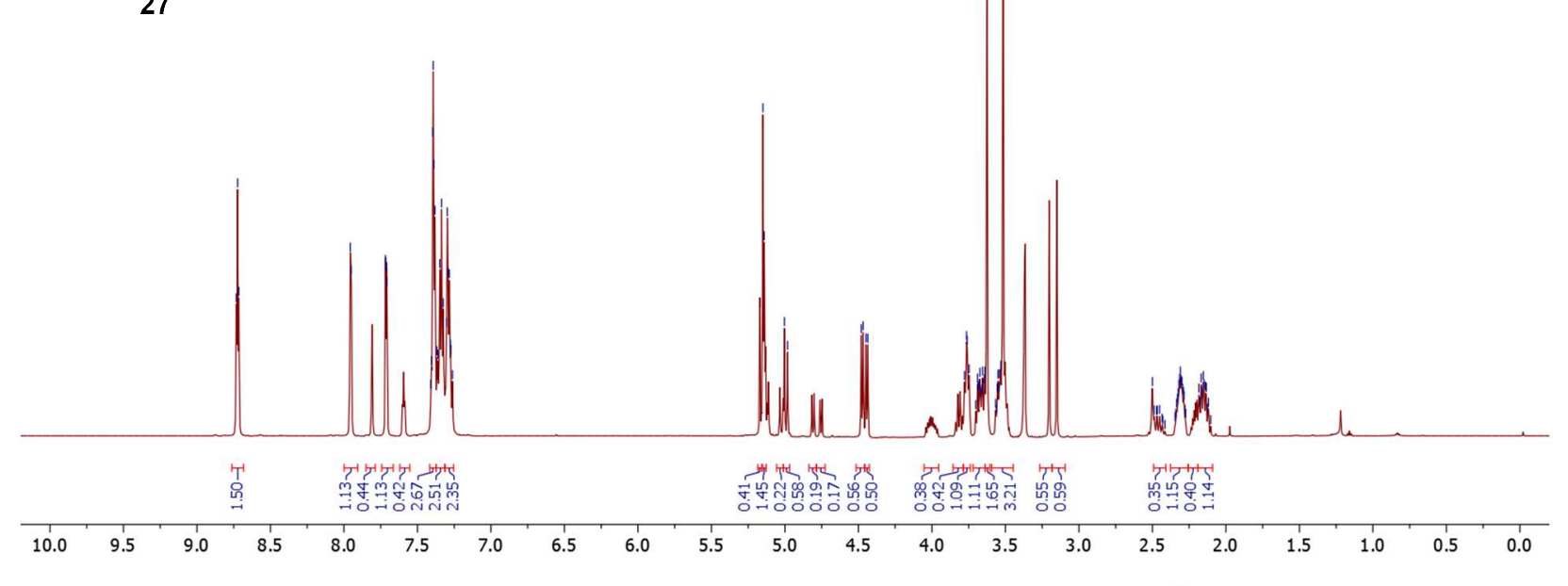

${ }^{13} \mathrm{C}$ NMR - $150.92 \mathrm{MHz}$ - DMSO-d6@308.0 K 等<smiles>COC(=O)[C@H]1[C@@H](c2ccnc(C(F)(F)F)c2)CCN1C(=O)[O-]</smiles>

27

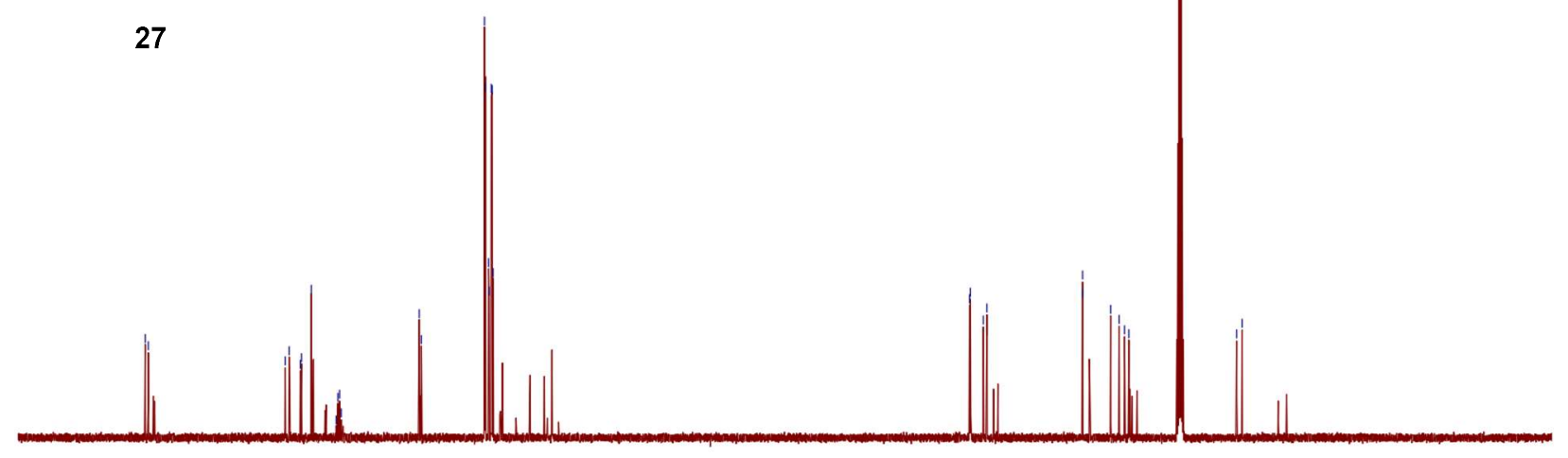




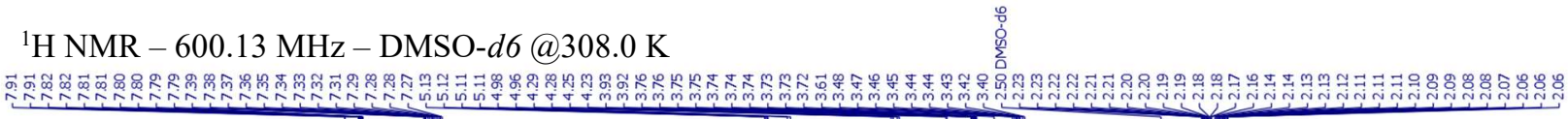<smiles>COC(=O)[C@H]1[C@@H](c2cnc(F)c(OC)c2)CCN1C(=O)O</smiles>

28

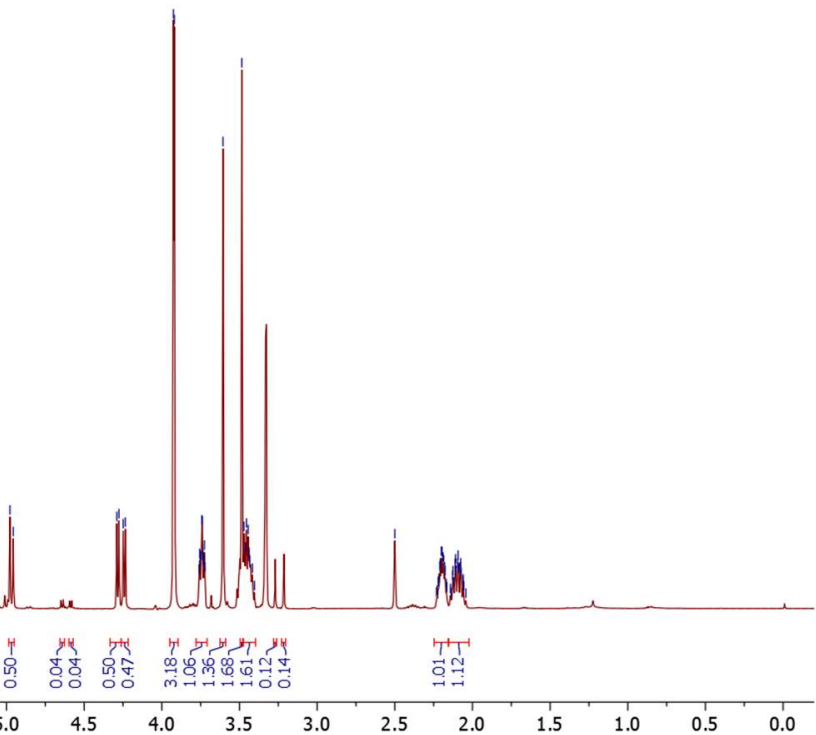

${ }^{13} \mathrm{C}$ NMR - $150.92 \mathrm{MHz}$ - DMSO-d6@308.0 K

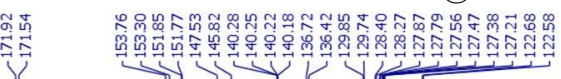<smiles>COC(=O)[C@H]1[C@@H](c2cnc(F)c(OC)c2)CCN1C(=O)O</smiles>

28

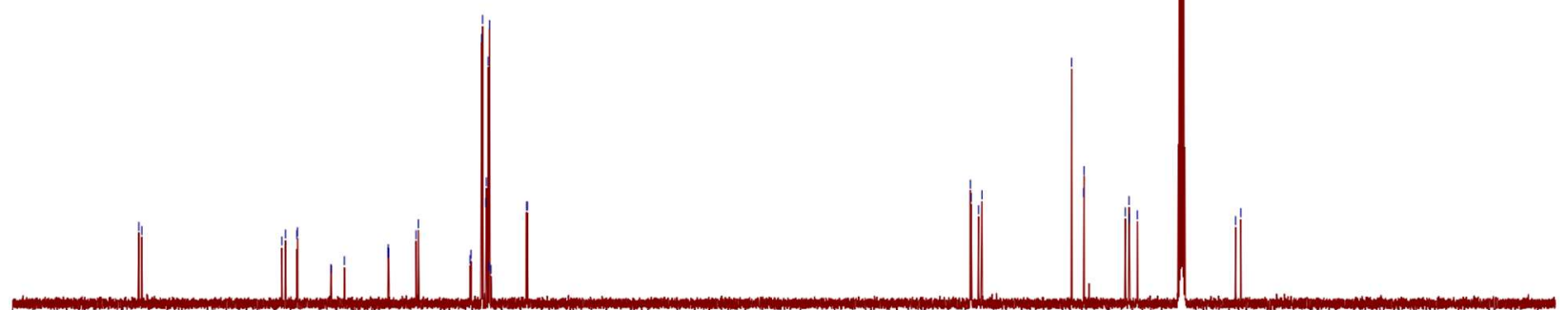

180

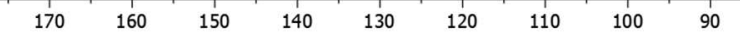

80

$60,50,40$

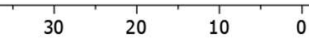




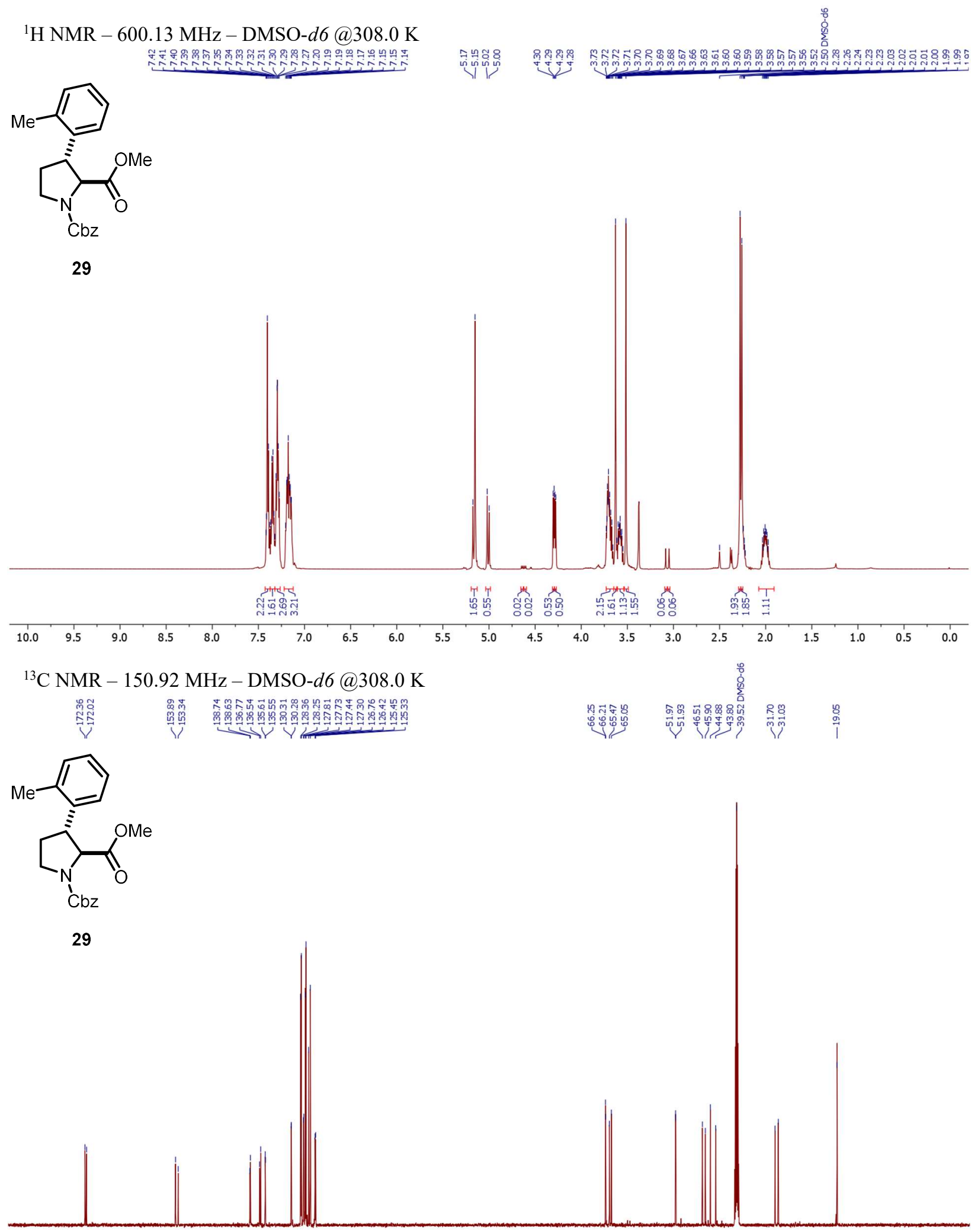

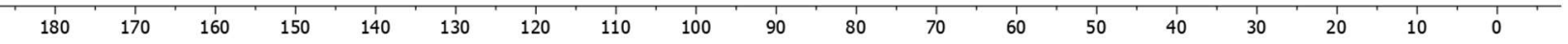




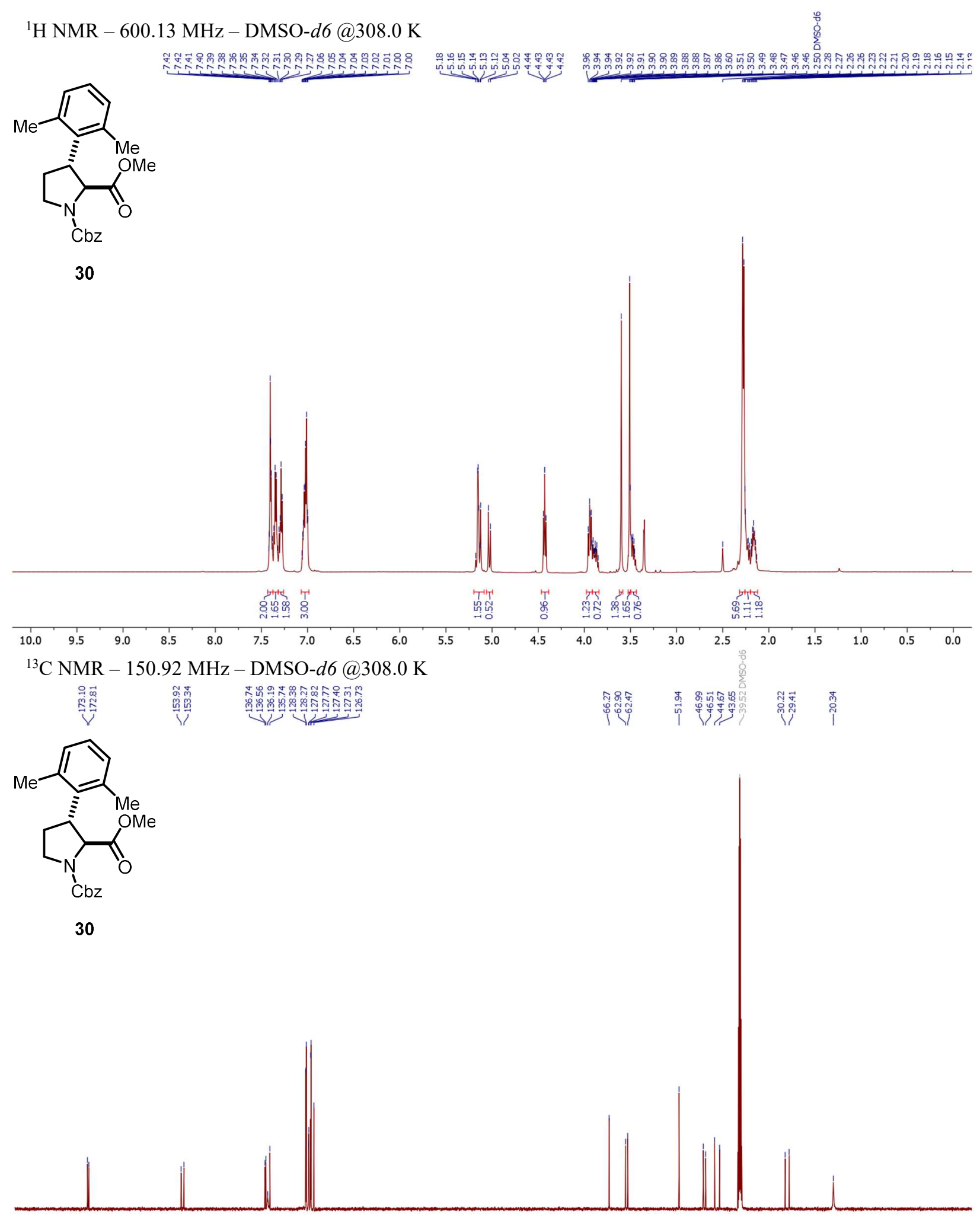

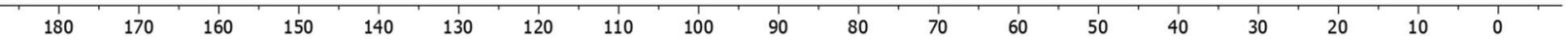




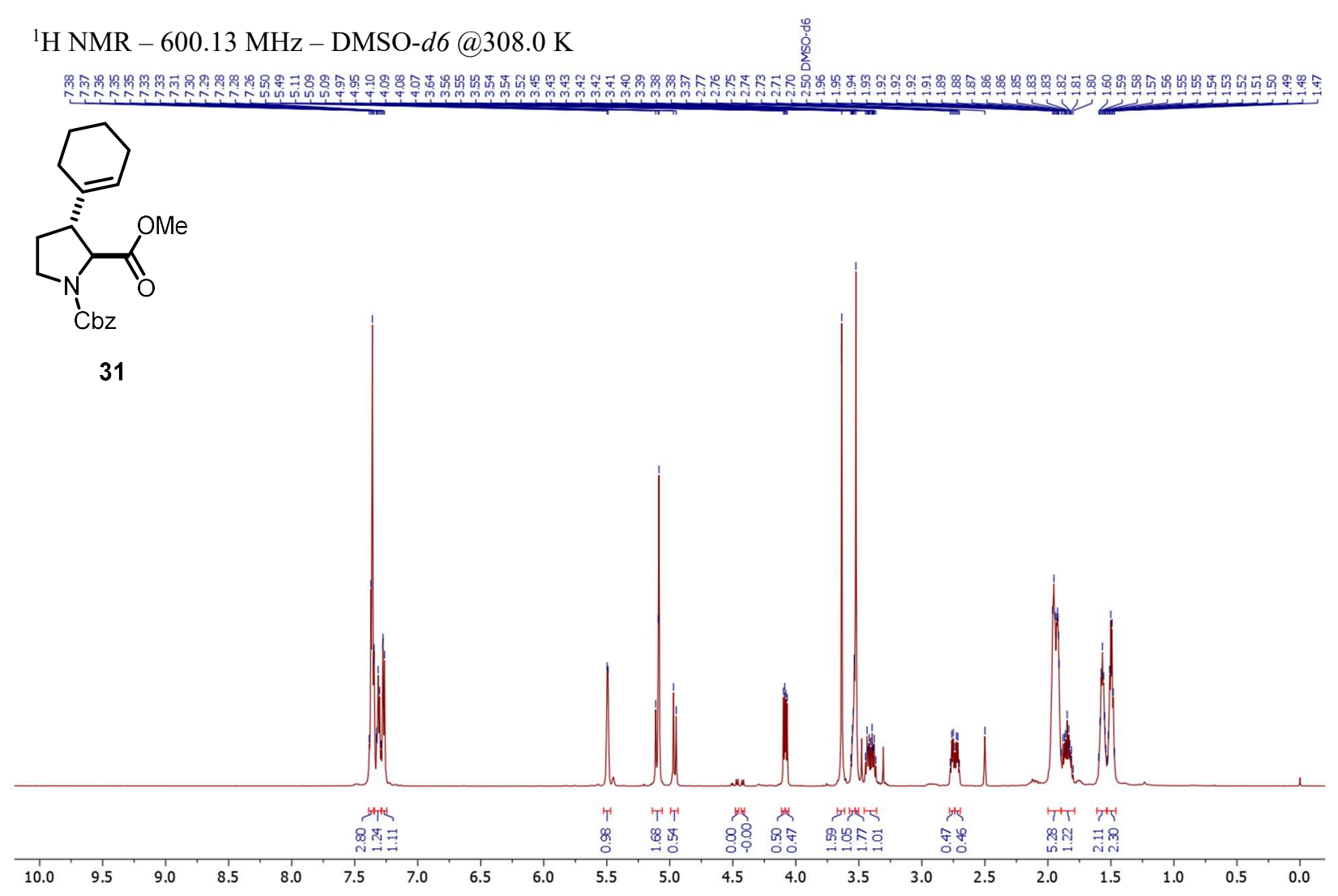

${ }^{13} \mathrm{C}$ NMR - 150.92 MHz - DMSO-d6@308.0 K
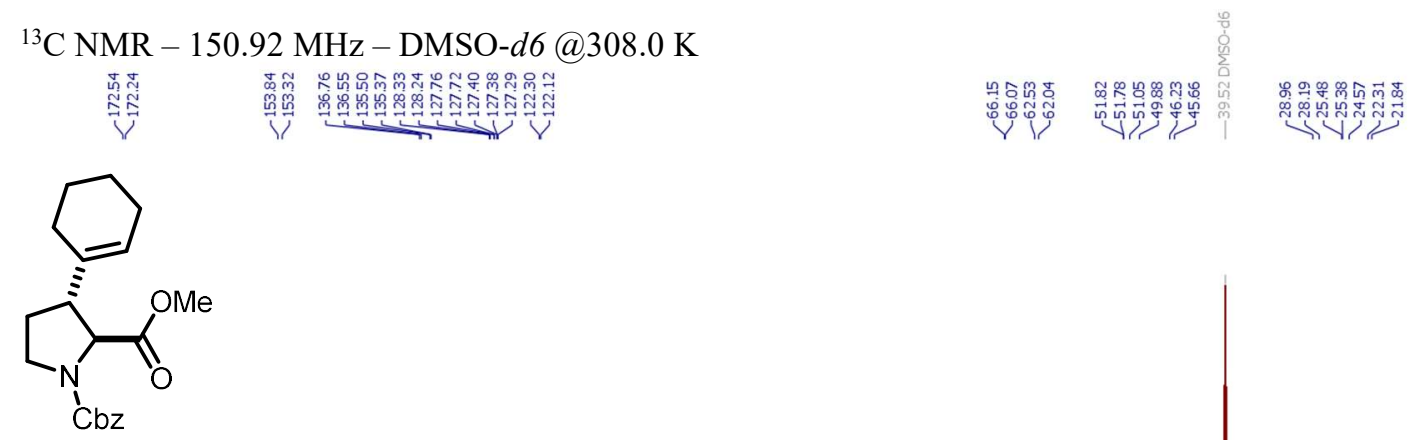

31
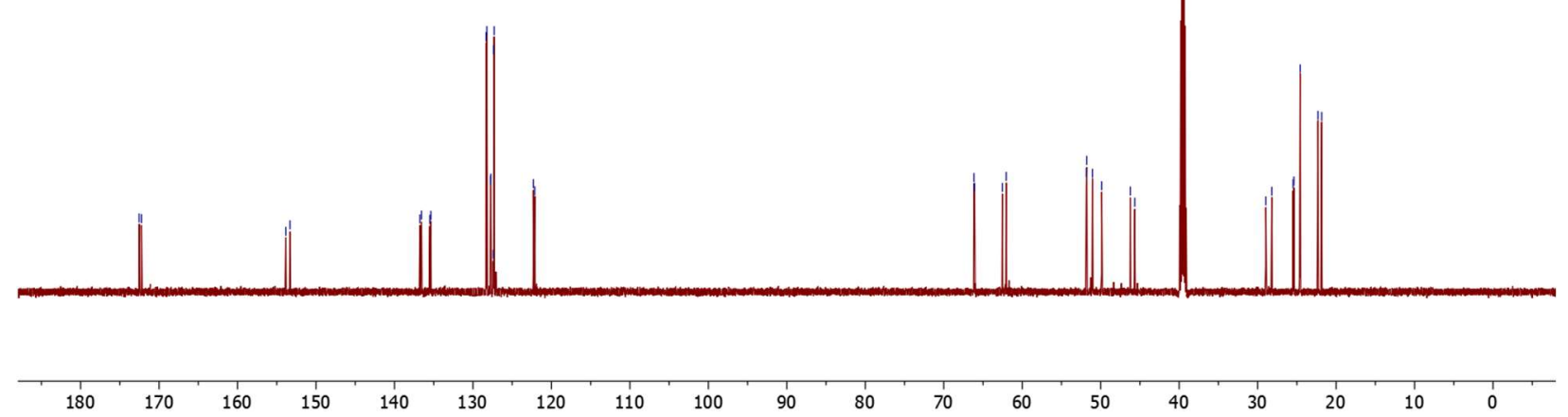